\title{
Studies of chemomechanical gels and collective behavior in chemical systems
}

\author{
Zhaoyang Huang \\ West Virginia University
}

Follow this and additional works at: https://researchrepository.wvu.edu/etd

\section{Recommended Citation}

Huang, Zhaoyang, "Studies of chemomechanical gels and collective behavior in chemical systems" (2009). Graduate Theses, Dissertations, and Problem Reports. 2840.

https://researchrepository.wvu.edu/etd/2840

This Dissertation is protected by copyright and/or related rights. It has been brought to you by the The Research Repository @ WVU with permission from the rights-holder(s). You are free to use this Dissertation in any way that is permitted by the copyright and related rights legislation that applies to your use. For other uses you must obtain permission from the rights-holder(s) directly, unless additional rights are indicated by a Creative Commons license in the record and/ or on the work itself. This Dissertation has been accepted for inclusion in WVU Graduate Theses, Dissertations, and Problem Reports collection by an authorized administrator of The Research Repository @ WVU.

For more information, please contact researchrepository@mail.wvu.edu. 


\section{Studies of Chemomechanical Gels}

and

\section{Collective Behavior in Chemical Systems}

Zhaoyang Huang

DISSERTATION

Submitted to the Eberly College of Arts and Sciences at

West Virginia University in Partial Fulfillment of the Requirements for the Degree of

Doctor of Philosophy

in

Chemistry

Kenneth Showalter, Ph.D., Chairman

Charles Jaffé, Ph.D.

Terry Gullion, Ph.D.

Kung K. Wang, Ph.D.

Boyd F. Edwards, Ph.D.

Morgantown, West Virginia

2009

Keyword: Stimuli Responsive Gel, Chemomechenical Oscillator, Chemomechanical Walker, BZ Reaction, Quorum Sensing, Kuramoto Synchronization

Copyright 2009 Zhaoyang Huang 


\title{
ABSTRACT
}

\section{Studies of Chemomechanical Gels and Collective Behavior in Chemical Systems}

\author{
Zhaoyang Huang
}

The synthesis, structure and properties of stimuli responsive gels are reviewed. A flow through reactor is constructed by attaching a secondary chamber to the output of a continuously stirred tank reactor (CSTR). The dynamical behavior of the primary and secondary chambers is characterized by carrying out the bromate-sulfite-ferrocyanide reaction in the reactors. The dynamical behavior of the secondary chamber was found to mimic that of the primary chamber. In addition, the secondary chamber offers spatially homogeneous and non-stirred $\mathrm{pH}$ oscillations for chemomechanical oscillators and walkers. Chemomechanical oscillators are obtained by placing poly(NIPAAm-co-MAAc) gel rods of diameters less than $530 \mu \mathrm{m}$ into the secondary chamber of the flow through reactor. The swelling ratio of the gel rods during each oscillation cycle is dependent on the rod diameter. Gel rods of smaller diameters exhibited larger swelling ratios. The linear swelling ratio of a $50 \mu \mathrm{m}$ diameter rod is $80 \%$ and close to the equilibrium swelling ratio. Buckling is observed for gel rods with large swelling ratios. A chemomechanical walker is constructed by adding two capillary legs to a rod comprised of poly(NIPAAm-co-MAAc) gel. The walker moves in a forward-backwardforward pattern, with movement that is highly directional. The translational direction is independent of the surface upon which the walker moves.

Quorum sensing refers to density-dependent gene-transcription regulation phenomena that occurs in certain bacteria colonies. The gene transcription can be turned on at high cell densities and off at low cell densities. A similar transition is observed in locally and globally coupled chemical systems. In a locally coupled system, ferroin-loaded beads are 
positioned to form a quasicircular monolayer covered by catalyst-free BZ solution. The catalyst BZ solution is composed of $0.10 \mathrm{M} \mathrm{NaBr}, 0.20 \mathrm{M} \mathrm{MA}, 0.60 \mathrm{M} \mathrm{H}_{2} \mathrm{SO}_{4}$ and varied concentration of $\mathrm{NaBrO}_{3}$. For solutions with $\mathrm{NaBrO}_{3}$ concentrations between $0.24 \mathrm{M}$ and $0.30 \mathrm{M}$, chemical waves are observed only in groups with a bead number larger than a critical value. A transition from steady state to oscillatory behavior occurs when the size of the group increases. This transition has the features of a dynamical quorum sensing transition. Numerical simulations indicate that the quorum sensing transition is associated with a decrease in the loss of activator to the solution. In a globally coupled system, large populations of ferroin-loaded beads are stirred in a batch reactor at different stirring rates. A transition from steady state to oscillatory behavior is observed when the number density of the beads is increased at high stirring rate. A Kuramoto synchronization transition, where oscillators gradually synchronize as the number density increases, is observed at low stirring rate. Numerical simulations were in accord with both transitions observed experimentally at the corresponding exchange rates. 


\section{Dedication}

To Yuping, the best wife in the world

To David and Erica, the most precious gift from God

To my mom and dad, the greatest parents in the world 


\section{Acknowledgements}

Having completed this work, I want to express my gratitude to many people. First and foremost, I would like to thank my research advisor and mentor, Dr. Kenneth Showalter, for providing a great working atmosphere in which I could pursue my research and also for putting together a talented group of researchers with whom I have been able to work. It is Dr. Showalter who brings me an exciting opportunity to study polymer gels and nonlinear dynamics and offers me fantastic training in science and scientific writing. I also express my gratitude to Dr. Kung K. Wang, Dr. Charles Jaffé, Dr. Terry Gullion, Dr. Mark E. Koepke and Dr. Boyd F. Edwards for their direction and for evaluating my dissertation.

My sincere appreciation goes to Dr. Mark Tinsley, Dr. Eugene Mihaliuk, Dr. Annette Taylor, Dr. Aaron Steele and Dr. Yonghong Yang. Dr. Mark Tinsley impressed me with his research capability and inter-personal skills, which were extremely valuable to my $\mathrm{PhD}$ studies. His insights into experimental phenomena are always inspirational and his attitude to face challenges is always enthusiastic. I also owe Dr. Tinsley a debt of gratitude for reading through most chapters of this work and for providing valuable suggestions. Encouragement from Dr. Eugene Mihaliuk constitute a life-time treasure. Without his encouragement, there would not have been the birth of Virtuallab, instrument control and data analysis software. Dr. Annette Taylor taught me how to use the XPPAUT package to do numerical simulations and gave me valuable suggestions on the writing of Chapter 4 of this dissertation. The award winning template, which made the writing of this dissertation less painful and time efficient, is from Dr. Aaron Steele. Dr. Yonghong Yang spent the whole summer of 2003 teaching me organic synthesis, and since then I started building self-confidence in organic chemistry. The importance of the encouragement I received from these great people can not be overemphasized.

I want to express my gratitude to Dr. Yongjun Li and Dr. Marco Rivera for being my best friends with whom I shared joyous and painful moments during this research. Mr. Calistus Ngonghala was so nice to read through Chapters 4 and 5, and provided valuable suggestions. I also like to extend my gratitude to all my colleagues. They have been so nice and we have always had a friendly environment to work in. They include Dr. Jianxia Cui, Dr. Florin Chirila, Dr. Tabitha Chigwada, Fang Wang, Hua Ke and Dr. Ryoichi Aihara. 
I also want to thank Don Feathers, Sherman Adams and Allen Burns for their constant help in making all kinds of reactors and parts required for my experiments.

Finally I want to thank my family. My parents are the greatest and most unselfish parents in the world. They always care about their kids more than themselves. The endless love they have for their son makes my mom so brave that she came to America without even being able to understand or say an English word. To my brothers and sister, for their understanding that I could not take care of my parents due to the fact that I was in America. My wife, Yuping, gives me everyday support both spiritually and emotionally. She helps me to manage my financial difficulties, research challenges and in taking care of our son, David and daughter, Erica for so many years. Without her support and encouragement, I would not have been able to complete this program. 


\section{Contents}

Title Page

Abstract

Dedication $\quad$ iv

$\begin{array}{ll}\text { Acknowledgements } & \text { V }\end{array}$

1 Introduction $\quad 1$

2 Introduction to Polymer Gels 3

2.1 Introduction and a Brief History of Polymer Gels . . . . . . . . . . . . 3

2.2 Preparation of Polymer Gels . . . . . . . . . . . . . . . . 4

2.2.1 Radical (co)Polymerization and Crosslinking . . . . . . . . . . . 4

2.2.2 Radical Production Techniques . . . . . . . . . . . . . . 5

2.2 .3 Chemical Crosslinking . . . . . . . . . . . . . 7

2.2 .4 Physical Crosslinking . . . . . . . . . . . . . 8

2.3 Properties of Stimuli Responsive Gels . . . . . . . . . . . . . . . 8

2.3.1 Temperature Responsive Gels . . . . . . . . . . . . . . . . . . . 8

$2.3 .2 \mathrm{pH}$ Responsive Gels . . . . . . . . . . . . . . . . . 13

2.4 Engineering Aspects of Polymer Gels . . . . . . . . . . . . . . . . . 16

2.4 .1 Transport Kinetics of Gels . . . . . . . . . . . . . . . 16

2.4 .2 Mechanical Properties of Gels . . . . . . . . . . . . . . . . . . 20

2.5 Applications of Stimuli Responsive Gels . . . . . . . . . . . . . . . . . . 20 
3 Flow Through Reactor 35

3.1 Construction of the CSTR . . . . . . . . . . . . . 35

3.2 CSTR Derived Reactors . . . . . . . . . . . . . . . . . . 37

3.2 .1 One-side-fed-reactors . . . . . . . . . . . . . . . . 37

3.2 .2 Flow Through Reactor . . . . . . . . . . . . . . . . 37

3.3 The Bromate-Sulfite-Ferrocyanide System in a Flow Through Reactor . . . . . 38

3.3.1 Bromate-Sulfite-Ferrocyanide System . . . . . . . . . . . . . . . . 40

3.3.2 Behavior of Bromate-Sulfite-Ferrocyanide System in Reactor CSTR2C1 41

3.3.3 Behaviors of Bromate-Sulfite-Ferrocyanide System in Reactor CSTR2C3 45

3.3.4 Spatial Homogeneity of the Secondary Chamber for the Reactor Model

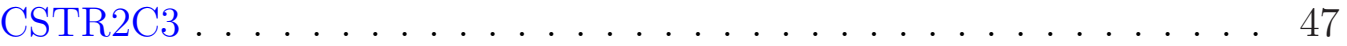

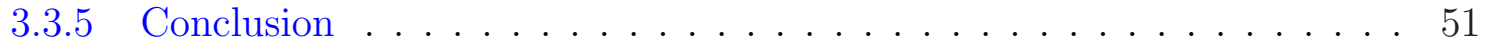

4 Chemomechanical Oscillators $\quad 54$

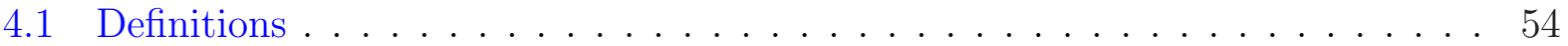

4.2 Construction of a Chemomechanical Oscillator . . . . . . . . . . . . . 55

4.2.1 Selection of the $\mathrm{pH}$ Responsive Gel . . . . . . . . . . . . . . . 55

4.2 .2 Selection of the $\mathrm{pH}$ Oscillator . . . . . . . . . . . . . . 55

4.3 Experimental Setup: Reactor . . . . . . . . . . . . . . . 56

4.4 Experimental Setup: Properties of the poly(NIPAAm-co-MAAc) Gel . . . . . 58

4.4.1 Synthesis of the poly(NIPAAm-co-MAAc) Gel . . . . . . . . . . . . 58

4.4 .2 Structure of the Gel . . . . . . . . . . . . . . . 58

4.4 .3 Visualization of the Gel . . . . . . . . . . . . 58

4.4 Equilibrium Swelling of the Gel . . . . . . . . . . . . . . . . . . 59

4.4.5 Dynamic Process of the Gel Swelling and the Swelling Kinetics . . . . . 62

4.4.6 Dynamic Process of the Gel Deswelling and the Deswelling Kinetics . . 65

4.5 Properties of the Chemomechanical Oscillator . . . . . . . . . . . . 68

4.5.1 Relationship between the pH Oscillator and the Chemomechanical Os-

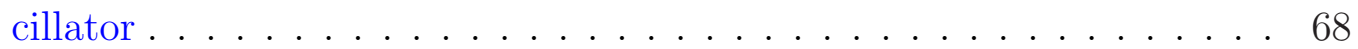

4.5.2 Buckling Behavior of the Gel . . . . . . . . . . . . . . . . 71 
4.6 Conclusion . . . . . . . . . . . . . . . . . . . . . . . . 73

$\begin{array}{lll}5 & \text { Chemomechanical Walkers } & 78\end{array}$

5.1 Introduction . . . . . . . . . . . . . . . . . . 78

5.2 Working Principles of the Chemomechanical Walker . . . . . . . . . . . . 79

5.2.1 Principles of the Ratchet and Pawl Mechanism . . . . . . . . . . . 79

5.2.2 Ratchet Mechanism of a Chemomechanical Walker . . . . . . . . . . 79

5.2.3 Stick-Slip Mechanism of Chemomechanical Walker . . . . . . . . . . 81

5.2.4 Asymmetric Surface Friction Design of a Chemomechanical Walker . . 84

5.3 Experimental Setup: Fabrication of the

Chemomechanical Walker . . . . . . . . . . . . . . . . . . . . . . . . 85

5.4 Experimental Setup: Reactor $\ldots \ldots \ldots \ldots \ldots$

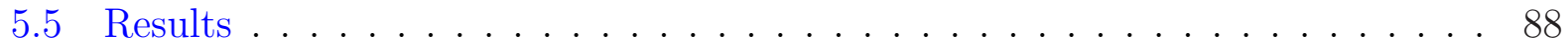

5.5.1 Movement of the Chemomechanical Walker . . . . . . . . . . . . 88

5.5.2 Speed of the Chemomechanical Walker . . . . . . . . . . . . 88

5.5.3 Selection of the Chemomechanical Walker Length . . . . . . . . . . . 90

5.6 Comparison with Other Chemomechanical Walkers . . . . . . . . . . . . . 94

5.7 Conclusion . . . . . . . . . . . . . . . . . . . . . . . 94

6 Spontaneous Wave Activity in Groups of Catalytic Particles 98

6.1 The Belousov-Zhabotinsky (BZ) Reaction . . . . . . . . . . . . . . . 98

6.2 Properties of Individual Catalyst-Loaded Particles . . . . . . . . . . . . . 100

6.2.1 Catalyst Particle Preparation . . . . . . . . . . . . . . . . . 100

6.2 .2 Properties of Uncoupled Particles _ . . . . . . . . . . . . . . . 100

6.3 Experimental Results . . . . . . . . . . . . . . . . . . . . . . 102

6.3 .1 Experimental Setup . . . . . . . . . . . . . . . . . . . . . . 102

6.3.2 Results with Excitable System . . . . . . . . . . . . . . . . . 105

6.3 .3 Features of Target Waves . . . . . . . . . . . . . . . . . 107

6.3.4 Features of Broken Waves . . . . . . . . . . . . . . . . . . 110

6.4 Simulation Results . . . . . . . . . . . . . . . . . . . . . . 110

6.4.1 ZBKE Model of the Belousov-Zhabotinsky Reaction . . . . . . . . . 110 
6.4.2 Simulation Model for the Spatially Coupled Bead System . . . . . . . . 114

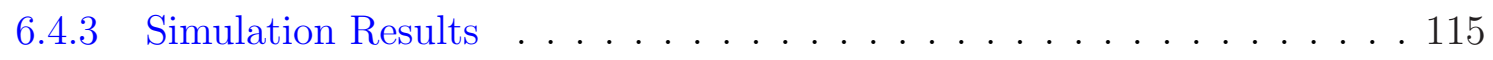

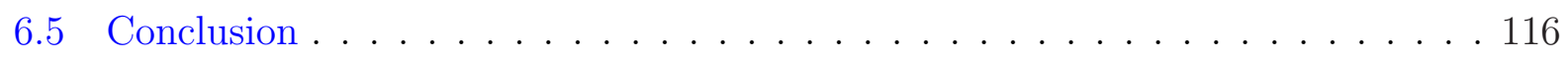

7 Collective Behavior of Globally Coupled Oscillators 122

7.1 Introduction . . . . . . . . . . . . . . . . . . . . 122

7.2 Experimental setup . . . . . . . . . . . . . . . . . 123

7.2.1 Bead Preparation and Oscillation Properties . . . . . . . . . . 123

7.2 .2 Experimental Setup . . . . . . . . . . . . . . . 124

7.2.3 Stirring Rate and Chemical Exchange Rate. . . . . . . . . . . 126

7.2 .4 Image Analysis . . . . . . . . . . . . . . . . . . 126

7.3 Experimental Results . . . . . . . . . . . . . . . . 127

7.3.1 Quorum Sensing Transition .................. 127

7.3 .2 Kuramoto Transition . . . . . . . . . . . . . . . . 128

7.4 Model Studies . . . . . . . . . . . . . . . . . . . . . . . . 130

7.4.1 Simulation Methods . . . . . . . . . . . . . 130

7.4.2 Quorum Sensing and Kuramoto Transitions . . . . . . . . . . . 134

7.4.3 Effect of Exchange Rate on Transitions . . . . . . . . . . . . . . . 134

7.5 Comparison between Locally and Globally Coupled Systems . . . . . . . . . . 137

7.6 Conclusion . . . . . . . . . . . . . . . . . . . 140

A Abbreviations for Monomers and Crosslinkers 145

$\begin{array}{ll}\text { B Design of Flow Through Reactor } & 148\end{array}$ 


\section{List of Tables}

2.1 Temperature responsive gels $\ldots \ldots \ldots \ldots \ldots$

$2.2 \mathrm{pH}$ responsive gels $\ldots \ldots \ldots \ldots \ldots \ldots$

3.1 Summary of the behaviors for flow through reactor (Model CSTR2C1) . . . 42

3.2 Summary of the behaviors for flow through reactor (Model CSTR2C3) . . . 45

4.1 Table for $\mathrm{pH}$ buffer preparation . . . . . . . . . . . . . . 60

6.1 The reaction scheme for the ZBKE model . . . . . . . . . . . . . . . . . . 112

6.2 Rate constants for the numerical simulations . . . . . . . . . . . . . 115

7.1 Quorum sensing transition and Kuramoto synchronization transition . . . . . 128

7.2 The value of parameters in the ZBKE model . . . . . . . . . . . . . . 133

7.3 Comparison between the spatial and stirred systems . . . . . . . . . . . . 140

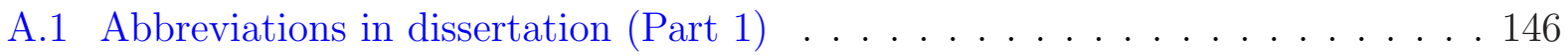

A.2 Abbreviations in dissertation $($ Part 2) $\ldots \ldots \ldots \ldots \ldots$ 


\section{List of Figures}

2.1 The structures for crosslinking reagents $\ldots \ldots \ldots \ldots \ldots \ldots$

2.2 The crosslinking process by the crosslinking reagent $\ldots \ldots \ldots \ldots$

2.3 Equilibrium swelling behavior of temperature responsive gels $\ldots \ldots$. . . . . 9

2.4 The structures for temperature responsive gel monomers . . . . . . . . . . . 11

2.5 Equilibrium swelling behavior of $\mathrm{pH}$ responsive gels . . . . . . . . . . . 15

2.6 The structures for typical anionic $\mathrm{pH}$ responsive gel monomers . . . . . . . 16

2.7 The structures for typical cationic $\mathrm{pH}$ responsive gel monomers . . . . . . . . 17

2.8 Comb network structure and regular network structure . . . . . . . . . . . 19

3.1 Design of a continuously stirred tank reactor $\ldots \ldots \ldots 36$

3.2 Reactors derived from a CSTR $\ldots \ldots \ldots \ldots \ldots$

3.3 Bifurcation diagram for bromate-sulfite-ferrocyanide reaction . . . . . . . . . 41

3.4 Typical behavior in the flow through reactor $(\mathrm{CSTR} 2 \mathrm{C} 1) \ldots \ldots \ldots$

3.5 Oscillation dependence on the secondary chamber volume for the flow through reactor $(\mathrm{CSTR} 2 \mathrm{C} 1) \ldots \ldots \ldots \ldots \ldots \ldots$

3.6 Typical behaviors of the BFS system in the flow through reactor (CSTR2C3) 46

3.7 Optical absorbance as a function of wavelength for the reactants in the bromatesulfite-ferrocyanide reaction $\ldots \ldots \ldots \ldots$

3.8 Spatial homogeneity analysis of the flow through reactor model CSTR2C3 . . 49

3.9 Sampling point positions of homogeneity examination by greyscale analysis . . 50

4.1 Experimental setup of the chemomechanical oscillator . . . . . . . . . . . . 57

4.2 Structure of the poly(NIPAAm-co-MAAc) gel . . . . . . . . . . . . . . 59 
4.3 Equilibrium length as a function of $\mathrm{pH}$ for the poly(NIPAAm-co-MAAc) gel at room temperature . . . . . . . . . . . . . . . . 61

4.4 Swelling kinetics at room temperature for poly(NIPAAm-co-MAAc) gel . . . . 63

4.5 Swelling process at room temperature for poly(NIPAAm-co-MAAc) gel . . . . 64

4.6 Deswelling process of $100 \mu \mathrm{m}$ poly(NIPAAm-co-MAAc) gel rod . . . . . . . . 65

4.7 Deswelling process of $537 \mu \mathrm{m}$ poly(NIPAAm-co-MAAc) gel rod in acid . . . 66

4.8 Deswelling process of $537 \mu \mathrm{m}$ poly(NIPAAm-co-MAAc) gel rod in pH 4 buffer 67

4.9 The relationships between the mechanical oscillation and the $\mathrm{pH}$ oscillation

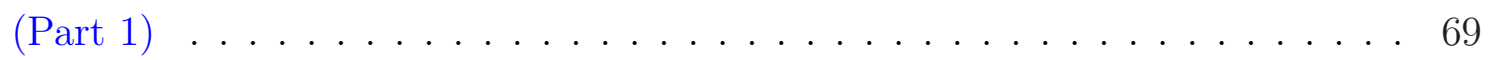

4.10 The relationships between the mechanical oscillation and the $\mathrm{pH}$ oscillation

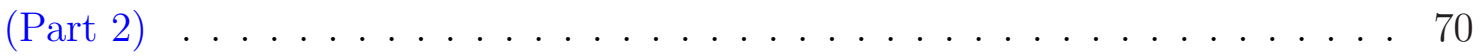

4.11 The swelling ratio dependence on the diameter of the gel rods . . . . . . . . 72

5.1 Principles of ratchet and pawl mechanism . . . . . . . . . . . . 80

5.2 Ratchet design of a chemomechanical walker . . . . . . . . . . . . 82

5.3 Stick-slip mechanism of a ratchet . . . . . . . . . . . . . 83

5.4 Asymmetric friction mechanism for a ratchet $\ldots \ldots \ldots \ldots$

5.5 Images of a chemomechanical walker leg and a grasshopper leg . . . . . . . 87

5.6 Shape of gel during one oscillatory period . . . . . . . . . . . . . . 89

5.7 Space-time plot of gel movement comprised of superimposed images. . . . . . . 90

5.8 The moving speed of the chemomechanical walker and pH vs. time . . . . . . 91

5.9 The path of the chemomechanical walker front . . . . . . . . . . . 92

5.10 Analysis of the movement of the chemomechanical walker . . . . . . . . . 93

6.1 Chemical oscillations during the BZ reaction . . . . . . . . . . . . . 99

6.2 Experimental behavior of individual beads . . . . . . . . . . . . . . 101

6.3 Oscillatory period of independent catalytic particles . . . . . . . . . . . 103

6.4 Bead size distribution . . . . . . . . . . . . . . . . . . . 104

6.5 Spontaneous formation of wave activity from groups of steady state beads . . 106

6.6 Dependence of wave period on bromate concentration . . . . . . . . . . 107 
6.7 Typical time series for a target wave and a spiral wave propagating in a group of beads. . . . . . . . . . . . . . . . . . . . . . . . . . . . . . 108

6.8 Snapshots of target wave behavior, and simple and complex broken waves . . 109

6.9 Sequence of bead initiations of a simple and complex broken wave . . . . . . 111

6.10 Schematic drawing of simulation model for coupled bead system . . . . . . . . 114

6.11 Transition from steady state to wave behavior as a function of group size . . . 116

6.12 Concentrations of $\mathrm{Br}^{-}$and $\mathrm{HBrO}_{2}$ as a function of the number of beads . . . 117

7.1 Bead size and oscillation period distributions . . . . . . . . . . . . . . . 124

7.2 Experimental setup of the stirred system _ . . . . . . . . . . . . 125

7.3 A typical plot of the fraction of oscillatory beads in the oxidized state determined with image analysis . . . . . . . . . . . . . . . . . . . . . . 127

7.4 Quorum sensing transition in the stirred system . . . . . . . . . . . . . 129

7.5 Kuramoto type synchronization transition in the stirred system . . . . . . . . 131

7.6 Numerical modeling setup . . . . . . . . . . . . . . . . . . . . . . . 133

7.7 Surface plots for maximum bromous acid concentration and order parameter K 135

7.8 Kuramoto synchronization transition and quorum sensing transition in globally coupled particle model . . . . . . . . . . . . . . . . . . . . 136

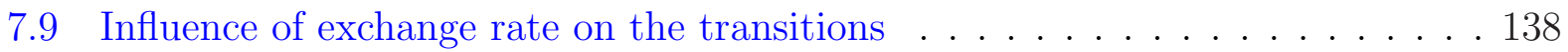

B.1 Dimensions for the secondary chamber of the flow through reactor . . . . . . 148

B.2 Main dimensions for the primary chamber of the flow through reactor . . . . . 149

B.3 Stirring wells for the primary chamber of the flow-through reactor . . . . . . 149

B.4 Inlet positions for the primary chamber of the flow through reactor $\ldots . . .150$ 


\section{Chapter 1}

\section{Introduction}

The observation that almost all living organisms have at least some components made of

polymer gels $[1,2,3]$ and the discovery of stimuli responsive gels in 1975 have inspired extensive research on characterizing gels. However, the application of gels in devices is still in its infancy when compared gels in nature. The first part of this work describes studies of the applications of chemomechanical polymer gels. Chapter 2 is a basic introduction of polymer gels, including the synthesis, structure, and properties of polymer gels, especially stimuli responsive gels. In Chapter 3, we describe a continuously stirred tank reactor and its derived reactors that will be used to construct chemomechanical oscillators and walkers. Chemomechanical oscillators are described in Chapter 4 while chemomechanical walkers are described in Chapter 5.

Communication among bacteria has been recognized only recently [4]. Experiments have revealed that they communicate with each other by releasing and detecting chemical species called autoinducers. We have studied how individuals interact with each other and a group and how collective behavior emerges between excitable and oscillatory particles. The second part of this work focuses on how the density of theses particles may change the behavior of individuals in dynamical systems. Studies carried out with locally and globally coupled catalyst-loaded beads are presented in Chapters 6 and 7 . 


\section{Bibliography}

[1] G. H. Pollack, "Is the cell a gel —and why does it matter?" Jpn. J. Physiol. 51, 649-660 (2001).

Online Version 1

[2] A. Frey-Wyssling, Submicroscopic morphology of protoplasm (Elsevier, Amsterdam, The Netherlands, 1953). 1

[3] Y. Osada and J.-P. Gong, "Soft and wet materials: Polymer gels," Adv. Mater. 10, 827837 (1998).

Online Version 1

[4] L. M. Jarvis, "Communal living," Chem. Eng. News 86, 15-23 (2008).

Online Version 1 


\section{Chapter 2}

\section{Introduction to Polymer Gels}

\subsection{Introduction and a Brief History of Polymer Gels}

Polymer gels are 3 dimensional polymer networks that contain a large amount of solvent [1]. Based upon the solvent, polymer gels can be classified as hydrogels (water is the solvent) and organogels (with an organic solvent). In general hydrogels are more extensively studied than the organogels. Generally polymer gels are soft, wet and elastic because of the large amount of solvent they contain.

Stimuli responsive gels are defined as gels that have a sharp volume-phase transition, a volume change up to several hundred times from the contracted state to the expanded state, in response to a slight change of environmental conditions. The environmental change can be temperature $[2,3,4,5,6], \mathrm{pH}[7,8,9,10,11,12,13,14,15,16,17,18,19]$, solvent composition [20, 21], light [22], electric field [23, 24, 25] or magnetic field [26]. Stimuli responsive gels may respond to more than one stimulus [27, 28, 29, 30, 31, 32, 33]. Stimuli responsive gels are considered to be intelligent or smart materials due to the responsive nature to the environmental condition. In the literature, an environmental sensitive gel is also used to refer to a stimuli responsive gel. For consistency and descriptive purposes, stimuli responsive gels will be used throughout the description.

The discovery of stimuli responsive gels was made by Tanaka in 1975 [34]. Tanaka observed a sharp volume change of a polyacrylamide gel that was placed in a mixture of acetone and water. The stimuli responsive gel inspired many studies of this new type of 


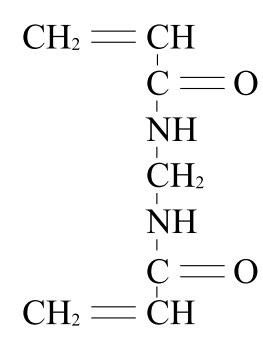

(a)

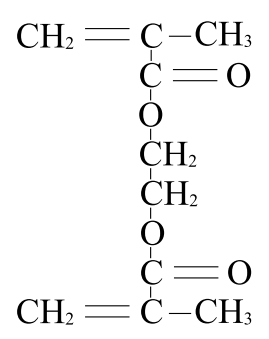

(b)

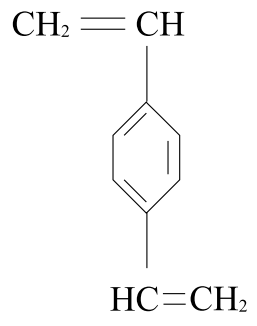

(c)

Figure 2.1. The structures for crosslinking reagents. (a) MBAA, (b) EGDMA, (c) DVB.

material. Since then further stimuli responsive gels have been invented and new applications of these gels have been developed.

\subsection{Preparation of Polymer Gels}

Polymer gels can be prepared by a number of techniques. In principle, any method that can crosslink the linear polymers to form temporary or permanent 3-D networks could be used. Here we review the most commonly used techniques.

\subsubsection{Radical (co)Polymerization and Crosslinking}

Radical (co)polymerization and crosslinking is the most widely used method to prepare polymer gels, which can be synthesized by an one or two-step process. In the one step process, the crosslinking is achieved at the same time as the polymerization, and for the two-step process, an additional crosslinking step is required. During crosslinking, covalent bonds are formed between the polymer chains and a permanent network structure is created.

Crosslinking reagents are generally bifunctional. During the polymerization process, they form links between the long polymer chains and allow the creation of the network structure. $N, N^{\prime}$-methylene bisacrylamide (MBAA), ethylenglycol dimethylacrylate (EGDMA) and divinylbenzene (DVB) are the most commonly used crosslinking reagents for gel preparation. Their structures are shown in Fig. 2.1.

Polymerization reactions occur by radical chain growth (addition) and the mechanism is 
well understood. The reaction can be characterized by a three-step process: chain initiation, chain propagation and chain termination.

$$
\begin{gathered}
I \rightarrow 2 R \cdot \\
R \cdot+M \rightarrow R M . \\
M_{x} \cdot+M \rightarrow M_{x+1} \cdot \\
M_{x} \cdot+M_{y} \cdot \rightarrow M_{x+y} \\
M_{x} \cdot+M_{y} \rightarrow M_{x}+M_{y}
\end{gathered}
$$

The chain initiation process can be considered as a two step process. For the first step, upon heating, UV illumination or redox reaction, the initiator decomposes and yields a pair of radicals (chemical specials with unpaired electrons). For the second step, chain radicals are produced between the primary radical $R$. and the monomer. These two steps are shown in Eq. 2.1 and Eq. 2.2, respectively. After initiation, chain radicals further react with the monomers and the chain grows, as shown by Eq. 2.3. If crosslinking reagents are present, secondary radicals form and react with the linear polymer chains randomly or simply react with the monomers to form branched chains. The network then grows by the crosslinking reaction, Fig. 2.2. Gelation occurs when the crosslinking density is beyond a critical point. The crosslinking process and the structure of the network are shown in Fig. 2.2. Chain radicals may also disappear through combination with each other or through disproportionation, as shown in Eqs. 2.4 and 2.5.

A variety of techniques to produce radicals can be found in the literature and offer great flexibility for the preparation of polymer gels.

\subsubsection{Radical Production Techniques}

Role of Oxygen in Gel Preparation. It is well known that oxygen inhibits the polymerization of vinyl monomers $[35,36,37]$. The inhibition effect of oxygen is due to the fact that oxygen is an effective radical scavenger and reacts with most radicals very rapidly [38]. The radicals produced are generally less reactive and cannot reinitiate the polymerization $[39]$. 


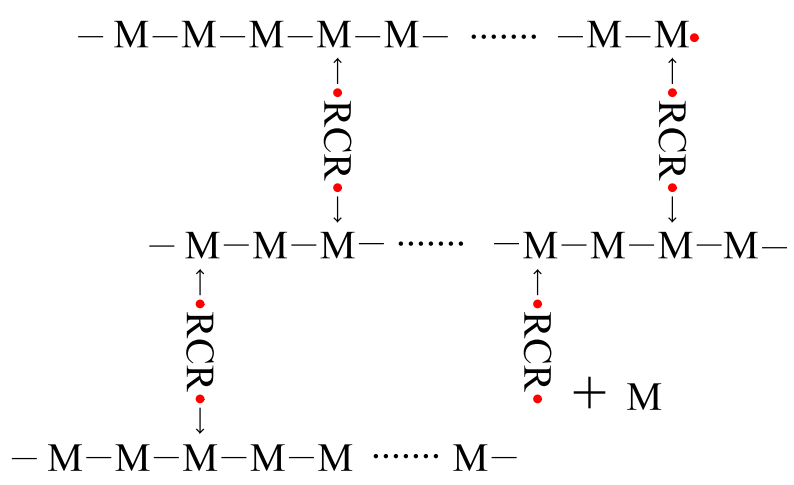

Figure 2.2. The crosslinking process by the crosslinking reagent. The secondary radicals $\cdot R C R$. formed by the reaction between the crosslinking reagent and the initiator species react with the monomer or with existing polymer chains to form network structures.

$$
M_{x} \cdot+O_{2} \rightarrow M_{x}-O-O
$$

On the other hand, oxygen can also serve as the initiator for the polymerization of styrene indirectly by peroxide radical formation $[37,40]$. Due to the inhibitory effect of oxygen, preparation of polymer gels from vinyl monomers by radical polymerization must be carried out under oxygen free conditions.

The role of oxygen during the photopolymerization and the reversible addition fragmentation chain transfer (RAFT) polymerization is still a topic of current investigation [41, 42]. The effect of oxygen on general polymer properties has yet to be fully characterized.

Thermoinitiation Polymerization. Thermoinitiation is the most widely used technique to prepare polymer gels due to its simplicity [15, 28]. Azo and peroxo compound are most common thermoinitiators found in the literature. Thermoinitiators are characterized by the half life, which is the time for the initiator to decompose by half, and 10 hour halflife temperatures are normally provided for convenient selection of the proper initiator or temperature.

Thermoinitiation polymerization is generally used with a mold method when gels of a controlled size and shape are required. Since the reaction temperature of polymerizations involving thermoinitiation is typically above $50^{\circ} \mathrm{C}$, nitrogen flushing and sealing of the solu- 
tions with the mold is required.

Redox Initiation Polymerization. Initiating polymerization through redox reactions is another popular technique to prepare polymer gels $[43,44]$. The reactions typically use ammonium persulfate (APS) or potassium persulfate (KPS) as the initiator and $N, N, N^{\prime}, N^{\prime}$ tetramethylethylenediamine (TEMED) as the accelerator. Compared with the thermoinitiated polymerization process, the redox reaction initiation process can be carried out at room temperature in aqueous medium and has better tolerance towards trace amount of oxygen. Therefore the reactions can be carried out between plates without complete sealing of the reactor. Large sizes of the gel and different geometrical shapes of gels can be prepared under less demanding conditions. The preparation of polyacrylamide gels is probably the most common example of this type of preparation [45].

Photo Initiation Polymerization. Photoinitiation polymerization is another technique used to prepare polymer gels $[46,47,48]$. Radicals are produced upon illumination with UV light to initiate the polymerization. By utilizing standard photolithography techniques, gels with precise patterns and two-dimensional shapes can be prepared, which is not possible with the two previously mentioned techniques. By using photoinitiation polymerization techniques, Bebee [47] fabricated polymer gel valves inside of a $50 \mu \mathrm{m}$ microfluidic channel. $\mathrm{Hu}$ et al [49] were able to construct two-dimensional responsive surface patterns.

\subsubsection{Chemical Crosslinking}

Besides the one-step polymerization crosslinking techniques, crosslinking after polymerization methods are also available to produce polymer gels bearing functional groups. Crosslinks can be formed by reactions between the functional groups on the polymer chains and the chemical crosslinker, generally a bifunctional or trifunctional compound [50]. The crosslinks can be stable or biodegradable depending on the crosslinker's structure, and the reaction may be catalyzed with enzymes.

High energy irradiation by gamma radiation rays or electron beams is an effective technique to produce radicals. Following polymerization, crosslinking can be initiated. Since 
radicals are formed during the reaction, an oxygen free environment is required. Poly(vinyl alcohol) [51], poly(ethylene oxide) $[52,53]$ and poly(acrylic acid) gels [50, 54] can be prepared this way.

\subsubsection{Physical Crosslinking}

Crosslinking produced by physical methods differs from that produced by chemical methods. Chemical crosslinking methods generally produce stable and permanent crosslinks, but physical techniques typically produce gels that have weak and temporary crosslinks. Physically crosslinked networks are not as stable as their chemical counterparts and can be weakened or broken by changing environmental conditions such as temperature or $\mathrm{pH}$. The crosslinks formed by physical methods include ionic interaction, physical entanglements, hydrogen bonding, hydrophobic interaction and crystallization.

Physical gels are generally prepared under mild conditions, either by simple mixing, heating-thawing cycles or by self-assembly (see review by Hennink and Nostrum [55]). We note that ultrasound irradiation has also been used to prepare gels [56].

\subsection{Properties of Stimuli Responsive Gels}

As introduced in Section 2.1, stimuli responsive gels can have an abrupt volume change in response to a small change in environmental conditions. Temperature and $\mathrm{pH}$ are the two most commonly used stimuli. The principles of the volume phase transition of temperature and $\mathrm{pH}$ responsive gels will be explained here.

\subsubsection{Temperature Responsive Gels}

The first stimuli responsive gel discovered involved response to temperature. For a small change of temperature, usually less than $1^{0} C$, a macroscopic gel volume transition, a solubleinsoluble transition [57], and a microscopic coil-globule transition [58] can be observed. The transition is characterized by direct measurement of the gel volume or weight, cloud

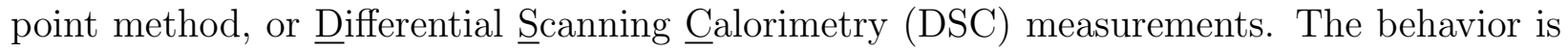




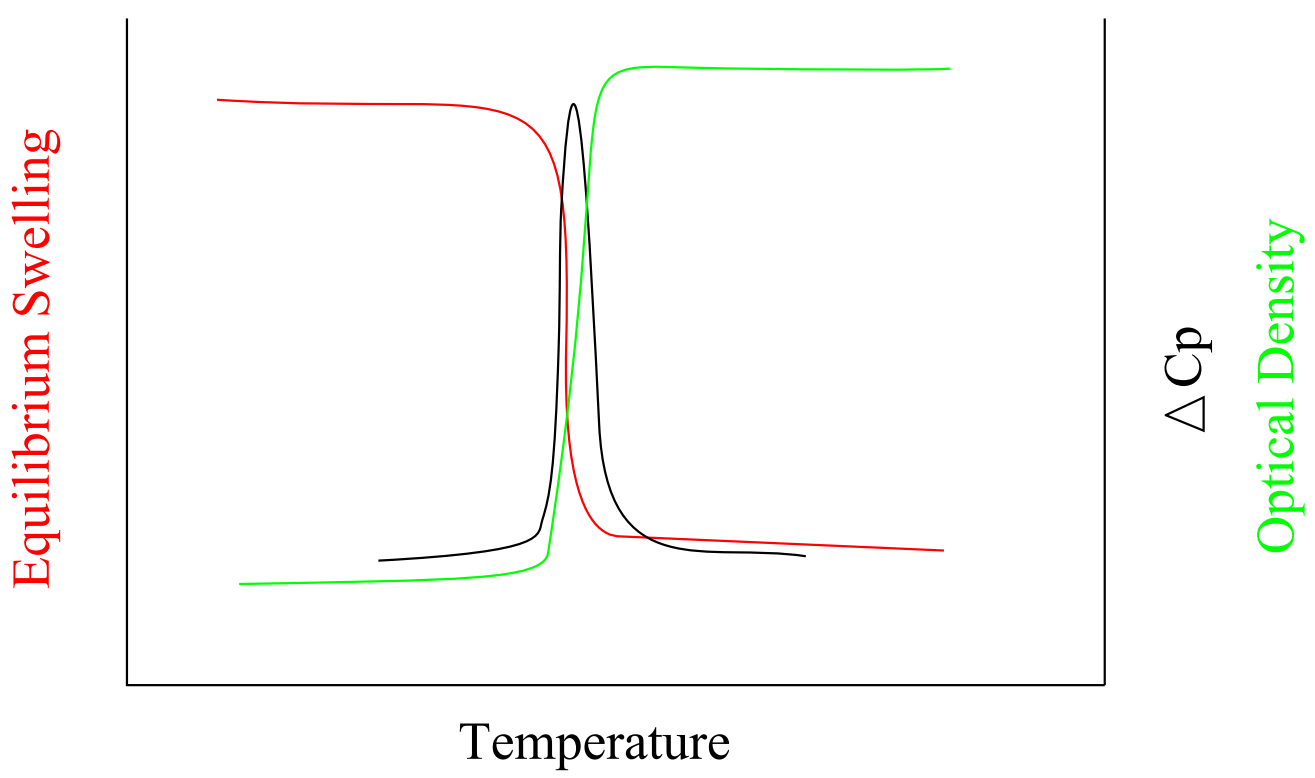

Figure 2.3. Equilibrium swelling behavior of temperature responsive gels. The red line represents the equilibrium swelling ratio at different temperatures. The green line represents the optical density. The black line represents the heat capacity change of the gel from Differential Scanning Calorimetry measurements.

schematically illustrated in Fig. 2.3.

Lower Critical Solution Temperature (LCST) is used to describe the abnormal solvation of temperature responsive polymers. Temperature responsive polymers precipitate at temperatures above the LCST and dissolve below the LCST. The LCST is the highest temperature for the dissolution of a polymer. Most polymers or chemicals precipitate at lower temperatures and dissolve at higher temperatures. The commonly found temperature responsive gels and their functional monomer and LCSTs are listed in Table 2.1.

NIPAAm and its comonomers form the largest class of temperature responsive gels. As the first temperature responsive gels discovered, they form the best studied type of stimuli responsive gels. All other temperature responsive gels share a similar chemical structure with NIPAAm. Flexible hydrophobic groups are connected to the network structure and are responsible for the collapse of the gel when the temperature is increased. The chemical structures of some temperature responsive gels are shown in Fig. 2.4.

Schild [57] and Peppas [66] provide similar qualitative explanations for the volume phase 
Table 2.1. Temperature responsive gels

\begin{tabular}{|l|l|l|l|}
\hline Functional monomer & Gel & LCST $\left({ }^{0} C\right)$ & refences \\
\hline NIPAAm & poly(NIPAAm) & $30-35$ & {$[6,27,57]$} \\
\hline NIPAAm & poly(NIPAAm-co-MAA) & $31-34$ & {$[28,44,46,59]$} \\
\hline NIPAAm & poly(NIPAAm-co-VP) & 40 & {$[32,60]$} \\
\hline NIPAAm & poly(NIPAAm-co-CIPAAm) & $24,27,29$ & {$[61,62]$} \\
\hline NIPAAm & poly(NIPAAm-co-BMA) & $20-30$ & {$[3]$} \\
\hline NIPAAm & poly(NIPAAm-co-AMPS) & 37 & {$[63]$} \\
\hline NIPAAm & poly(NIPAAm-co-AAc) & $30-50$ & {$[31,63,64,65]$} \\
\hline DEAAm & poly(DEAAm) & 25 & {$[3]$} \\
\hline DEAAm & poly(DEAAm-co-MA) & 55 & {$[6]$} \\
\hline APy & poly(APy) & 33,40 & {$[2,3]$} \\
\hline AA & poly(AA) & 25 & {$[27]$} \\
\hline AA & poly(AA-co-TMAAPAI) & 13,40 & {$[21]$} \\
\hline AcrNMP & poly(AcrNMP-co-MAA) & $22.00-88.11$ & {$[33]$} \\
\hline AcrNEP & poly(AcrNEP-co-MAA) & $19.60-92.30$ & {$[33]$} \\
\hline HPMA & poly(MA-(L)Pro-co-HPMA) & 20 & {$[5]$} \\
\hline VMEG & poly(VMEG) & 37 & {$[4]$} \\
\hline
\end{tabular}



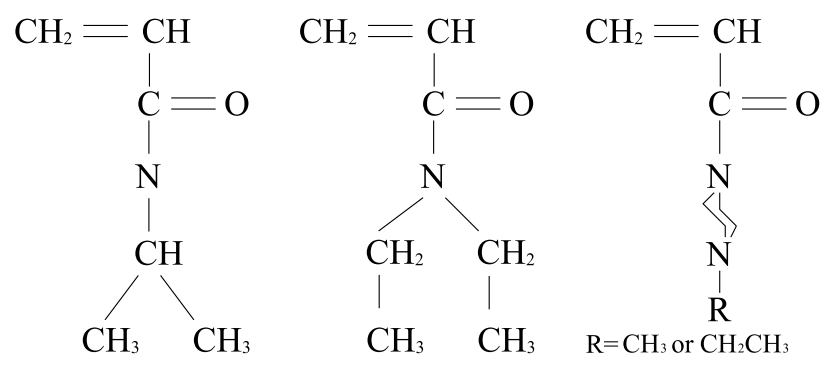

(a)

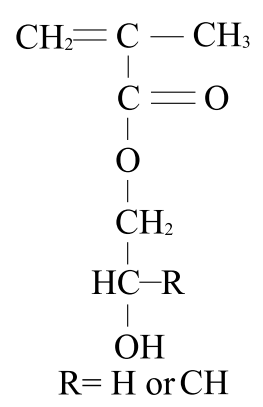

(d) (b)

(c)

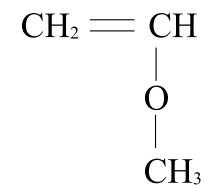

(f)

Figure 2.4. The structures for temperature responsive gel monomers. (a) NIPAAm, (b) DEAAm, (c) AcrNAP, (d) HAMA, (e) AAm, (f) VMEG. (See Appendix A for names of gel monomers.) 
transition of temperature responsive gels, which are summarized below. The swelling of the polymer gel can be treated as a mixing process between the polymer chains and the solvent. The solvation process of the stimuli responsive gel is different from that of most polymers and other chemicals in that the enthalpy $(\Delta H)$ for the temperature responsive gel mixing is slightly negative and the entropy $(\Delta S)$ is negative. Due to hydrophobic effects, water molecules reorient around the polar groups of NIPAAm and form more ordered structures. The reorientation process decreases the entropy. The Gibbs energy can be calculated from the entropy and enthalpy for a particular temperature by Eq. 2.7:

$$
\Delta G=\Delta H-T \Delta S
$$

We know for the solvation process, $\Delta H<0$ and $\Delta S<0$, and therefore we can expect a critical temperature

$$
T_{c}=\frac{\Delta H}{\Delta S}
$$

when $\Delta G$ is equal to zero. When the temperature is above $T_{c}, \Delta G$ is positive and the precipitation process is spontaneous. On the other hand, the solvation process is spontaneous at temperatures below $T_{c}$ with an associated negative $\Delta G$. If $\Delta S$ is large enough, the Lower Critical $\underline{\text { Solution }}$ Temperature (LCST) can be observed and a sharp volume phase transition results.

It is important to be able to tune the $T_{c}$ for biomedical applications such as drug delivery. As well as by appropriate selection of the functional polymer, the LCST of the temperature responsive gels can be finely tuned by incorporating additional comonomers or by varying the ratio between the hydrophilic and hydrophobic segments [67]. Increase of the LCST of the temperature responsive gels can be realized by increasing the fraction of the hydrophilic segments of the gel. However, too many hydrophilic groups may cause the temperature sensitivity to decrease and even cause the LCST to disappear completely. On the other hand, the incorporation of hydrophobic groups can decrease the LCST and the transition will become sharper. This is clearly demonstrated by the copolymerization of $N$-isopropylacrylamide with acrylamide and $N$-tert butylacrylamide [68]. For the copolymer between $N$-isopropylacrylamide and acrylamide, the LCST will increase by increasing the amount of acrylamide (when below 20\%) in the copolymer due to the hydrophilic nature 
of the acrylamide. On the other hand, the incorporation of hydrophobic $N$-tert butylacrylamide decreases the LCST of the copolymer gel with $N$-isopropylacrylamide. The larger the fraction of $N$-tert butylacrylamide in the copolymer, the lower the LCST of the copolymer. Using these techniques, the range of LCST can be greatly expanded and covers from $0^{0} \mathrm{C}$ to $55^{0} \mathrm{C}$. Terminal modification by hydrophobic or hydrophilic groups has a more pronounced effect on the LCST of the temperature responsive gels than the incorporation of the comonomer.

\subsection{2 $\mathrm{pH}$ Responsive Gels}

pH responsive gels are polyelectrolytes that consist of weak acids or weak bases. Their responses to $\mathrm{pH}$ are described in Fig. 2.5. The volume phase transition $\mathrm{pH}$ is close to the pKa of the corresponding monomer units. For poly(carboxylic acid) gels, the contracted state can be acquired when the $\mathrm{pH}$ is below the pKa of the gel, while a swollen gel can be acquired when the $\mathrm{pH}$ is above pKa. The chemical structures of commonly used acidic monomers are shown in Fig. 2.6. The opposite behavior can be observed for poly(base) gels, which contain units with amine groups. At low $\mathrm{pH}$, the amine groups are protonated and the volume is larger, while at high $\mathrm{pH}$, the amine groups are neutral and the gel volume is smaller. The basic monomers or units used to prepare $\mathrm{pH}$ responsive gels are shown in Fig. 2.7. For the poly(ampholyte) gels, consisting of monomers that contain both acidic and basic groups, two swollen states appear at high and low pHs and a contracted state appears at intermediate $\mathrm{pH}$. The commonly found $\mathrm{pH}$ responsive gels are listed in Table 2.2.

The mechanism for $\mathrm{pH}$ responsive gels is more complex than the temperature responsive gels. Many factors contribute to the equilibrium swelling, such as hydrophilicity of the polymer chains and Donnan equilibrium.

For acidic hydrogels, for an example poly(acrylic acid) gel, the carboxylic group on the polymer chains will be protonated when the $\mathrm{pH}$ is below the pKa. On protonation, the chain becomes neutral and the gel becomes less hydrophilic. The hydrophilicity decrease also makes the gel less soluble in water, and therefore the total volume is smaller when the gel is in the contracted state. When the $\mathrm{pH}$ is above the pKa, deprotonation occurs and renders the polymer chains more hydrophilic. Water then enters the polymer network and 
Table 2.2. $\mathrm{pH}$ responsive gels

\begin{tabular}{|l|l|l|l|}
\hline Functional Unit & Gel & Phase Transition $\mathrm{pH}$ & refences \\
\hline AAc & poly(AAc) & 4.0 & {$[11]$} \\
\hline AAc & poly(AAc-co-HEMA) & $5.38,5.25$ & {$[29,47]$} \\
\hline AAc & poly(NIPAAm-co-AAc) & 4,5 & {$[31,64,65]$} \\
\hline AAc & poly(AAs-co-AAc) & $5.0-8.0$ & {$[15]$} \\
\hline MAAc & poly(MAAc) & 5.5 & {$[46]$} \\
\hline MAAc & poly(MAAc-co-EG) & 5.7 & {$[14]$} \\
\hline MAAc & poly(NIPAAm-co-MAAc) & $5.2-5.7$ & {$[28,44,46]$} \\
\hline MAAc & poly(HEMA-co-MAAc) & & {$[7,8]$} \\
\hline MAAc & poly(MAAc-g-EG) & 5.6 & {$[14,48]$} \\
\hline AAm & poly(AAm) & & {$[9,69]$} \\
\hline 4 -VP & poly(NIPAAm-co-4-VP) & 3.5 & {$[32]$} \\
\hline DMAEMA & poly(AMAE-co-DMA) & $5.5-6.5$ & {$[13]$} \\
\hline AMPS,MAPTA & poly(AMPS-co-MAPTA) & & {$[70]$} \\
\hline SDM & poly(SDM-co-DMAAm) & 7.2 & {$[71]$} \\
\hline poly(silamine) & poly(silamine) gel & $8.1-9.7$ & {$[72]$} \\
\hline
\end{tabular}




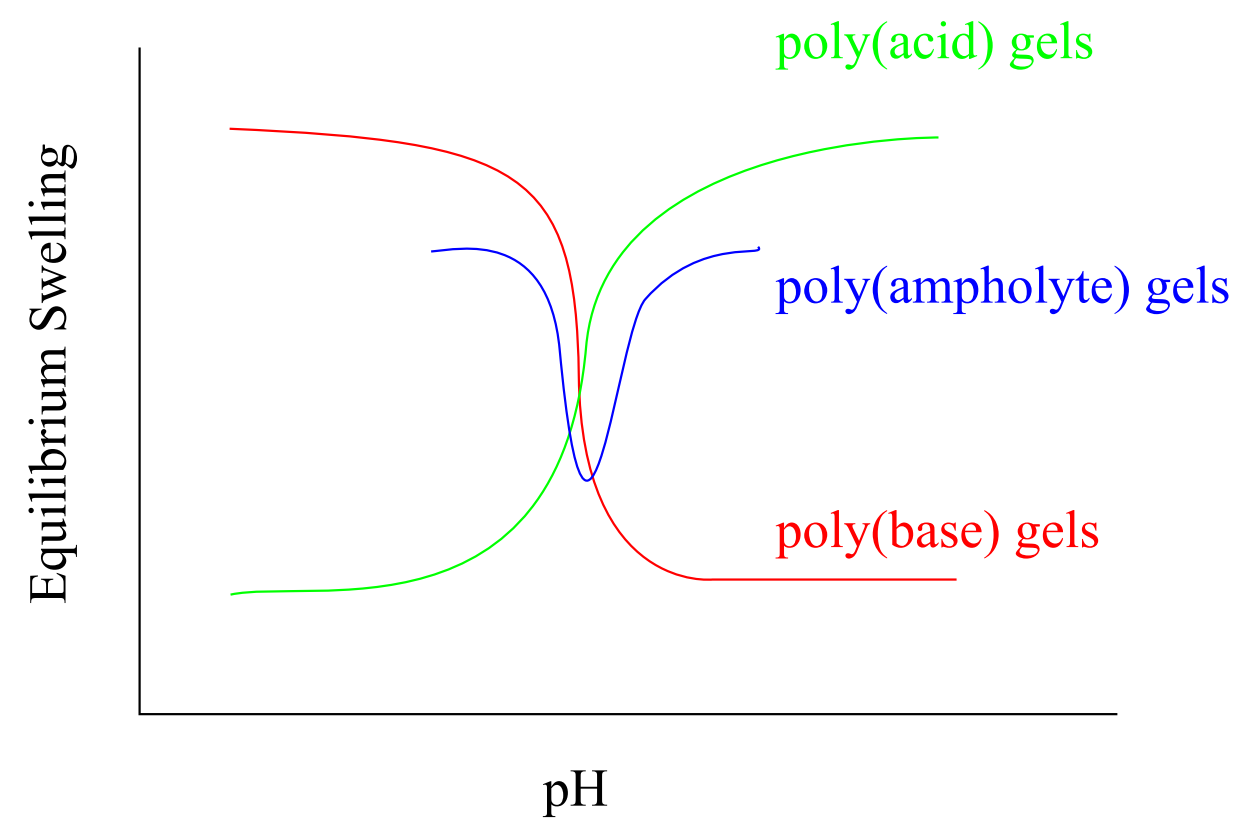

Figure 2.5. Equilibrium swelling behavior of $\mathrm{pH}$ responsive gels. Green, red and blue lines represent poly(acid) gels, poly(base) gels and poly(ampholyte) gels, respectively.

cause the gel to swell. A similar analysis applies to the poly(base) gels.

In addition to the hydrophilic considerations, Donnon equilibrium also contributes to the swelling of gels. At equilibrium, counterions will be attracted by the charges of the polymer chains and the concentration of the counterions will always be higher than in the surrounding solution. Thus the osmotic pressure will cause water to move into the gel and cause it to swell. At different $\mathrm{pHs}$, the charge density of the polymer chains determines the extent of swelling of the gel.

It is important to have the capability of finely tune the phase transition $\mathrm{pH}$ to suit specific applications. For an example, drug delivery gels that target the gastrointestinal (GI) tract should be quite different from gels that target the stomach, due to the difference of the $\mathrm{pH}$ environment. Systematic studies have been carried out for poly(acids) and poly(bases) $[13,15]$. For poly (acrylic acid) gels, the incorporation of hydrophobic groups into the polymer chains causes the phase transition $\mathrm{pH}$ to shift higher [15]. The longer the length of the alkyl chain or the larger the fraction of the hydrophobic component, the larger the shift. For the poly(base) gels, the opposite behavior is observed: the introduction of hydrophobic groups 


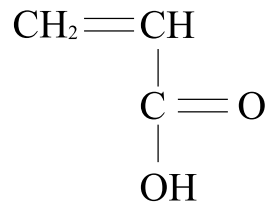

(a)

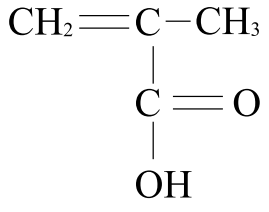

(b)

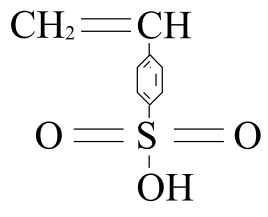

(c)

Figure 2.6. The structures for typical anionic pH responsive gel monomers. (a) AAc, (b) MAAc, (c) p-styrene sulfonic acid. (See Appendix A for names of gel monomers.)

shifts the phase transition to lower $\mathrm{pH}$. For PDMAEMA, the higher the hydrophobicity of $n$-alkylmethacrylate, the larger the shift of the $\mathrm{pH}[13]$.

\subsection{Engineering Aspects of Polymer Gels}

Although advances have been made in generating desired properties of polymer gels, there are still limitations in terms of the kinetics and mechanical properties of these materials. Here we review the progress made to improve the response time and the mechanical strength of stimuli responsive gels.

\subsubsection{Transport Kinetics of Gels}

The mass transport inside the gel depends on diffusion only, which inherently is a slow process and is strongly length-scale dependent. The transport process is described by Eq. $2.8[43,73,74]$ :

$$
\tau=\frac{l^{2}}{\pi^{2} D}
$$

where $\tau$ is the characteristic response time, $l$ is the characteristic length of the gel, and $D$ is the collective diffusion coefficient of the polymer network.

Equation 2.8 tells us that the response time to external stimuli can be long, especially for large gels. It may take hours or even days for large gels to reach equilibrium. The slow kinetics significantly limits their applications, especially in the fields of sensors, medical applications and artificial muscles. However, studies have been carried out to improve the 


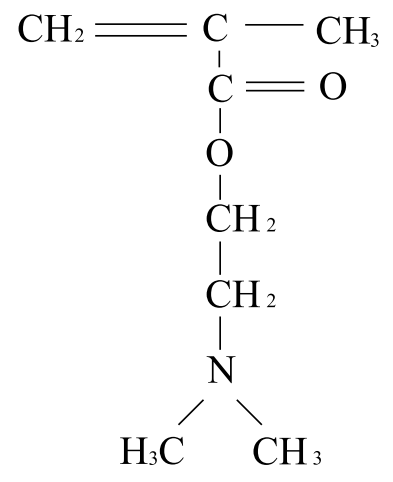

(a)

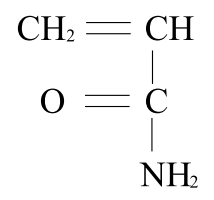

(c)

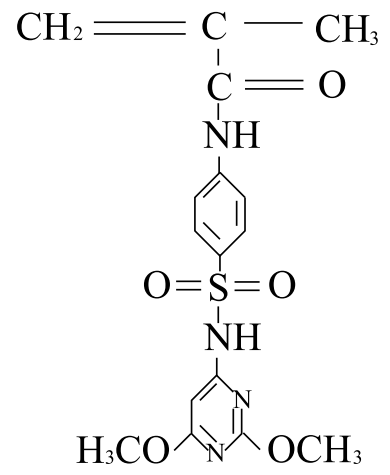

(b)

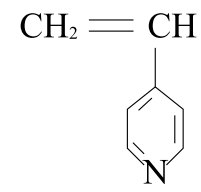

(d)

Figure 2.7. The structures for typical cationic pH responsive gel monomers. (a) DMAEMA, (b) SDM, (c) AAm, (d) 4-Vinyl Pyridine. (See Appendix A for names of gel monomers.) 
response time of the gels. The two major routes that have been taken to decrease the response time are modification of the macroscopic structure of the gel and modification of the microscopic structure at molecular level.

\section{Modification of the Macroscopic Gel Structure}

From Eq. 2.8, we know the response time is proportional to the square root of the length scale of the gel. Manufacturing gels with decreased size is straightforward in order to decrease the response time of the gel. For example, gel valves of micrometer size [47] have been fabricated and the gel response time has been significantly improved. Micrometer or even nanometer gel beads $[74,75]$ have also been prepared for drug delivery with a response time of a few seconds. Similarly, very thin gel slabs [49] also have much faster response times than the bulk gels.

Although gels with decreased size can greatly improve the response time, such gels are a challenge to fabricate and, in addition, these gels typically suffer from low mechanical strength. Fabrication techniques for decreased gel size have mainly relied on photolithography procedures. Gels fabricated by this method are limited to effectively two-dimensional structures. Microbeads or nanobeads can be fabricated by suspension polymerization or emulsion polymerization techniques.

\section{Modification of the Microscopic Gel Structure}

As discussed above, diffusion is the primary mass transport within the gel, so a different mass transport mechanism could also possibly decrease the gel response time. Instead of relying on diffusion through the polymer network, porous gels utilize capillary action to fill the network with the corresponding solvent, causing the gel to swell. For the capillary action to be the dominant mass transport within the gel, the pore size must be at least several micrometers. Porous gels can be prepared by a variety of techniques [76, 77, 78, 79, 80, 81, 82, 83].

Incorporating a porosigen (pore-forming reagent) during the synthesis of a gel permits pores to be formed on removal of the porosigens [79, 80, 81, 84, 85]. Micrometer sized sucrose [85], sodium chloride [84] and poly(ethylene glycol) [80] are commonly used as porosigens and can be easily removed by dissolving the porosigen on immersing the gel in water. 


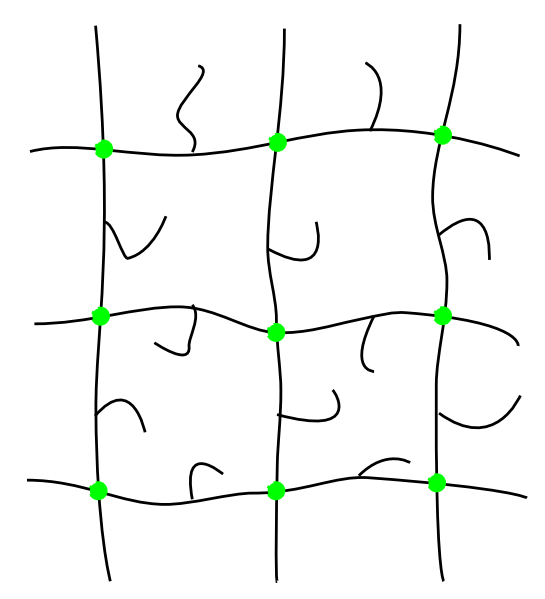

(a)

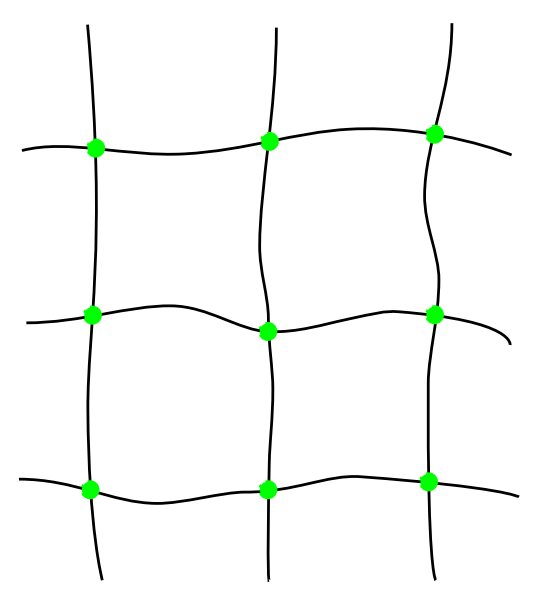

(b)

Figure 2.8. Comb network structure and regular network structure. (a) Comb-type network, (b) Regular network.

Foaming techniques $[82,86]$ are also utilized to prepare porous gels. By simply introducing a foaming reagent and foaming stabilizer into the original gel preparation, pores can be formed during the polymerization process.

Phase separation and crosslinking individual hydrogels can be used to prepare porous poly(HEMA) or poly(NIPA) gels and gels with active functional groups on the surface respectively [76, 77, 83, 87].

Instead of a regular network structure, comb-type gels introduce free chains in the main network and the response time can be accelerated by more than a factor of ten [88, 89, 90]. A comb-type and a regular network structure are shown in Fig. 2.8. During the shrinking process of a gel, the outer layer will shrink first. For a gel with a regular network structure, after shrinking, the pore size becomes smaller and a hard skin layer forms that prevents the release of internal solvent. The response time for the entire gel is consequently long. For the comb-type gel, the hydrophobic chains aggregate to form hydrophobic channels that facilitate the release of solvent. 


\subsubsection{Mechanical Properties of Gels}

Compared with natural polymer gels, the mechanical strength of man-made gels is much weaker than their biological counterparts. A dramatic example is cartilage, which is an extremely strong biopolymer [91]. By tuning the micro structure of artificial polymer gels, their typically low mechanical strength can be significantly improved. The general method that is used is to distribute the forces evenly across the polymer network, so the force applied to each individual chain is the same [92].

One technique is to introduce slideable crosslinks between the polymer chains [93]. Polyrotaxane chains have bulky end groups and $\alpha$-cyclodextrin $(\alpha-\mathrm{CD})$ molecules are sparsely distributed across the long chains. During the gelation step, $\alpha$-CDs are covalently linked to form figure-eight structures. Upon stretching, the pulley action effectively distributes the forces across the whole network, thus leading to an increased mechanical strength.

A second technique involves a double network structure [94]. After gel formation, a second network is formed with low crosslinking density. The chains are loosely dangling inside the first network. The force applied can be effectively reduced by the loosely bound chains of the second network.

Another technique depends on a hybrid structure composed of nanometer clay particles and polymer chains [95]. Two factors contribute to the stretchability and high mechanical strength of this system: many polymer chains on the surface of the clay particles can even out the forces applied and the crosslinking density is more homogeneous than in a regular gel.

\subsection{Applications of Stimuli Responsive Gels}

Because of their intrinsic properties, stimuli responsive gels have been widely used in pharmaceuticals, separations, microfluidic devices and artificial muscles.

Due to their responsive characteristics, stimuli responsive gels can be used as carriers for drug delivery applications. For an example, using $\mathrm{pH}$ responsive gels, drugs can be preloaded outside the body and release can be triggered by the $\mathrm{pH}$ at specific sites such as the stomach. Since the release is strictly regulated by $\mathrm{pH}$, premature release will not occur at sites such 
as the gastrointestinal (GI) tract. Other than the specificity of the destination as a drug carrier, the stimuli responsive gels can regulate drug release according to the body state by sensing the concentration of indicator molecules $[16,96]$. By incorporating the glucose oxidase (GOD) enzyme, the $\mathrm{pH}$ responsive gels can sense the concentration change of glucose, and the added feedback mechanism can thus regulate the release of insulin into the blood of a diabetes patient.

In response to environmental conditions, stimuli responsive gels not only have macroscopic volume changes but can also have microscopic pore size variations. Based on the variation of pore size, stimuli responsive gels can be used for separation of molecules of different sizes $[4,69]$. In the expanded state, the pore size of a gel is larger, and both small and large molecules can diffuse into the network. By changing the external conditions, the gel shrinks with the pore size becoming smaller. Large molecules will then be trapped within the gel and separation based on size can be achieved.

Fast response microscopic gel valves can be fabricated on microfluidic chips [47] or nanofluidic devices [97]. The on-off switching of the valves can be easily controlled by an external condition such as temperature. Due to the miniaturized design, these valves response with high speed. 


\section{Bibliography}

[1] Y. Osada and J. Gong, "Stimuli-responsive polymer gels and their application to chemomechanical systems," Prog. Polym. Sci. 18, 187-226 (1993).

Online Version 3

[2] Y. H. Bae, T. Okano, and S. W. Kim, "Insulin permeation through thermo-sensitive hydrogels," J. Control. Release 9, 271-279 (1989).

Online Version 3, 10

[3] T. Okano, Y. Bae, H. Jacobs, and S. Kim, "Thermally on-off switching polymers for drug permeation and release," J. Control. Release 11, 255-265 (1990).

Online Version 3, 10

[4] X. Huang, T. Akehata, H. Unno, and O. Hirasa, "Dewatering of biological slurry by using water-absorbent polymer gel," Biotechnol. Bioeng. 34, 102-109 (1989).

Online Version 3, 10, 21

[5] M. Yoshida, M. Asano, and M. Kumakura, "A new temperature-sensitive hydrogel with $\alpha$-amino acid group as side chain of polymer," Eur. Polym. J. 25, 1197-1202 (1989). Online Version 3, 10

[6] R. F. Freitas and E. Cussler, "Temperature sensitive gels as extraction solvents," Chem. Eng. Sci. 42, 97-103 (1987).

Online Version 3, 10

[7] L. Brannon-Peppas and N. A. Peppas, "Solute and penetrant diffusion in swellable polymers. IX. The mechanisms of drug release from $\mathrm{pH}$-sensitive swelling-controlled 
systems," J. Control. Release 8, 267-274 (1989).

Online Version 3, 14

[8] J. H. Kou, G. L. Amidon, and P. I. Lee, "pH-dependent swelling and solute diffusion characteristics of poly(hydroxyethyl methacrylatecomethacrylie acid) hydrogels," Pharm. Res. 5, 592-597 (1988).

Online Version 3, 14

[9] S. H. Gehrke and E. Cussler, "Mass transfer in pH-sensitive hydrogels," Chem. Eng. Sci. 44, 559-566 (1989).

Online Version 3, 14

[10] V. Kůdela, J. Vacík, and J. Kopeček, "Strong-acid membranes with enhanced hydrophilicity," J. Membrane Sci. 6, 123-131 (1980).

Online Version 3

[11] C. J. Crook, A. Smith, R. A. Jones, and A. J. Ryan, "Chemically induced oscillations in a pH-responsive hydrogel," Phys. Chem. Chem. Phys. 4, 1367-1369 (2002).

Online Version 3, 14, 54, 55, 68

[12] J. Heller and P. V. Trescgny, "Controlled drug release by polymer dissolution II: Enzyme-mediated delivery device," J. Pharm. Sci. 68, 919-921 (1979).

Online Version 3

[13] R. A. Siegel and B. A. Firestone, "pH-dependent equilibrium swelling properties of hydrophobic polyelectrolyte copolymer gels," Macromolecules 21, 3254-3259 (1988).

Online Version 3, 14, 15, 16

[14] M. Torres-Lugo and N. Peppas, "Molecular design and in vitro studies of novel pHsensitive hydrogels for the oral delivery of calcitonin," Macromolecules 32, 6646-6651 (1999).

Online Version 3, 14 
[15] O. Philippova, D. Hourdet, R. Audebert, and A. Khokhlov, "pH-responsive gels of hydrophobically modified poly(acrylic acid)," Macromolecules 30, 8278-8285 (1997). Online Version 3, 6, 14, 15, 60

[16] S. Kang and Y. H. Bae, "A sulfonamide based glucose-responsive hydrogel with covalently immobilized glucose oxidase and catalase," J. Control. Release 86, 115-121 (2003).

Online Version 3, 21

[17] A. S. Lee, V. Bütün, M. Vamvakaki, S. P. Armes, J. A. Pople, and A. P. Gast, "Structure of pH-dependent block copolymer micelles: Charge and ionic strength dependence," Macromolecules 35, 8540-8551 (2002).

Online Version 3

[18] R. C. Sutton, L. Thai, J. M. Hewitt, C. L. Voycheck, and J. S. Tan, "Microdomain characterization of styrene-imidazole copolymers," Macromolecules 21, 2432-2439 (1988). Online Version 3

[19] Z. Sideratou, D. Tsiourvas, and C. Paleos, "Quaternized poly(propylene imine) dendrimers as novel pH-sensitive controlled-release systems," Langmuir 16, 1766-1769 (2000).

Online Version 3

[20] Y. Hirokawa and T. Tanaka, "Volume phase transition in a nonionic gel," J. Chem. Phys. 81, 6379-6380 (1984).

Online Version 3

[21] S. Katayama and A. Ohata, "Phase transition of a cationic gel," Macromolecules 18, 2781-2782 (1985).

Online Version 3, 10

[22] Y. Yu, M. Nakano, and T. Ikeda, "Directed bending of a polymer film by light," Nature 425, 145-145 (2003).

Online Version 3 
[23] T. Tanaka, I. Nishio, S.-T. Sun, and S. Ueno-Nishio, "Collapse of gels in an electric field," Science 218, 467-469 (1982).

Online Version 3

[24] R. Kishi and Y. Osada, "Reversible volume change of microparticles in an electric field," J. Chem. Soc. Faraday Trans. 1. 85, 655 - 662 (1989).

Online Version 3

[25] A. Fernández-Nieves, A. Fernández-Barbero, F. J. de las Nieves, and B. Vincent, "Motion of microgel particles under an external electric field," J. Phys.: Condens. Matter. 12, 3605-3614 (2000).

Online Version 3

[26] G. Filipcsei, I. Csetneki, A. Szilágyi, and M. Zrínyi, Magnetic Field-Responsive Smart Polymer Composites, volume 206 of Adv. Polym. Sci., 137-189 (Springer, Berlin, 2007). Online Version 3

[27] T. Tanaka, "Phase transitions in gels and a single polymer," Polymer 20, 1404-1412 (1979).

Online Version 3, 10

[28] C. S. Brazel and N. A. Peppas, "Synthesis and characterization of thermo- and chemomechanically responsive poly(N-isopropylacrylamide-co-methacrylic acid) hydrogels," Macromolecules 28, 8016-8020 (1995).

Online Version 3, 6, 10, 14, 55, 58, 59, 60, 67

[29] B. Zhao and J. Moore, "Fast pH- and ionic strength-responsive hydrogels in microchannels," Langmuir 17, 4758-4763 (2001).

Online Version 3, 14

[30] C. Jones and L. Lyon, "Synthesis and characterization of multiresponsive core-shell microgels," Macromolecules 33, 8301-8306 (2000).

Online Version 3 
[31] C. Nolan, M. Serpe, and L. Lyon, "Thermally modulated insulin release from microgel thin films," Biomacromolecules 5, 1940-1946 (2004).

Online Version 3, 10, 14

[32] V. Pinkrah, M. Snowden, J. Mitchell, J. Seidel, B. Chowdhry, and G. Fern, "Physicochemical properties of poly(N'-isopropylacrylamide-co-4-vinylpyridine) cationic polyelectrolyte colloidal microgels," Langmuir 19, 585-590 (2003).

Online Version 3, 10, 14

[33] L. Gan, G. Roshan Deen, X. Loh, and Y. Gan, "New stimuli-responsive copolymers of N-acryloyl-N'-alkyl piperazine and methyl methacrylate and their hydrogels," Polymer 42, 65-69 (2001).

Online Version 3, 10

[34] R. Dagani, "Intelligent gels," Chem. Eng. News 75, 26-37 (1997). Online Version 3

[35] P. Flory, Principles of polymer chemistry (Cornell University Press, Ethaca, NY, USA, 1953). $5,62,71$

[36] C. E. Barnes, "Mechanism of vinyl polymerization. I. role of oxygen," J. Am. Chem. Soc. 67, 217-220 (1945).

Online Version 5

[37] H. S. Taylor and A. A. Vernon, "The photo-polymerization of styrene and vinyl acetate," J. Am. Chem. Soc. 53, 2527-2536 (1931).

Online Version 5, 6

[38] B. Maillard, K. Ingold, and J. Scaiano, "Rate constants for the reactions of free radicals with oxygen in solution," J. Am. Chem. Soc. 105, 5095-5099 (1983).

Online Version 5

[39] C. C. Price, "Some aspects of the mechanism of addition polymerization," Ann. N.Y. Acad. Sci. 44, 351-370 (1943).

Online Version 5 
[40] F. A. Bovey and I. M. Kolthoff, "The mechanism of emulsion polymerizations. III. oxygen as a comonomer in the emulsion polymerization of styrene," J. Am. Chem. Soc. 69, 2143-2153 (1947).

Online Version 6

[41] A. O'Brien and C. Bowman, "Impact of oxygen on photopolymerization kinetics and polymer structure," Macromolecules 39, 2501-2506 (2006).

Online Version 6

[42] Z. Zhang, J. Zhu, Z. Cheng, and X. Zhu, "Reversible addition-fragmentation chain transfer (RAFT) polymerization of styrene in the presence of oxygen," Polymer 48, 4393-4400 (2007).

Online Version 6

[43] T. Tanaka, E. Sato, Y. Hirokawa, S. Hirotsu, and J. Peetermans, "Critical kinetics of volume phase transition of gels," Phys. Rev. Lett. 55, 2455-2458 (1985). Online Version 7, 16

[44] S. Zhou and B. Chu, "Synthesis and volume phase transition of poly(methacrylic acidco-N-isopropylacrylamide) microgel particles in water," J. Phys. Chem. B 102, 13641371 (1998).

Online Version 7, 10, 14

[45] E. Harlow and D. Lane, Using antibodies: A laboratory manual (Cold Spring Harbor Laboratory Press, Plainview, NY, USA, 1999). 7

[46] J. Zhang and N. Peppas, "Synthesis and characterization of $\mathrm{pH}-$ and temperaturesensitive poly(methacrylic acid)/poly(N-isopropylacrylamide) interpenetrating polymeric networks," Macromolecules 33, 102-107 (2000).

Online Version 7, 10, 14

[47] D. J. Beebe, J. S. Moore, J. M. Bauer, Q. Yu, R. H. Liu, C. Devadoss, and B.-H. Jo, "Functional hydrogel structures for autonomous flow control inside microfluidic chan- 
nels," Nature 404, 588-590 (2000).

Online Version 7, 14, 18, 21

[48] R. Bashir, J. Z. Hilt, O. Elibol, A. Gupta, and N. A. Peppas, "Micromechanical cantilever as an ultrasensitive pH microsensor," Appl. Phys. Lett. 81, 3091-3093 (2002). Online Version 7, 14

[49] Z. Hu, Y. Chen, C. Wang, Y. Zheng, and Y. Li, "Polymer gels with engineered environmentally responsive surface patterns," Nature 393, 149-152 (1998).

Online Version 7, 18

[50] W. Hennink and C. van Nostrum, "Novel crosslinking methods to design hydrogels," Adv. Drug. Deliver. Rev. 54, 13-36 (2002).

Online Version 7, 8

[51] N. Peppas and E. Merrill, "Crosslinked poly(vinyl alcohol) hydrogels as swollen elastic networks," J. Appl. Polym. Sci. 21, 1763-1770 (1977).

Online Version 8

[52] P. Kofinas, V. Athanasssiou, and E. Merrill, "Hydrogels prepared by electron irradiation of poly(ethylene oxide) in water solution: Unexpected dependence of crosslink density and protein diffusion coefficients on initial PEO molecular weight," Biomaterials 17, 1547-1550 (1996).

Online Version 8

[53] J. Stringer and N. Peppas, "Diffusion of small molecular weight drugs in radiationcrosslinked poly(ethylene oxide) hydrogels," J. Control. Release 42, 195-202 (1996). Online Version 8

[54] E. Jabbari and S. Nozari, "Swelling behavior of acrylic acid hydrogels prepared by $\gamma$ radiation crosslinking of poly(acrylic) acid in aqueous solution," Eur. Polym. J. 36, 2685-2692 (2000).

Online Version 8 
[55] W. R. Gombotz and S. Wee, "Protein release from alginate matrices," Adv. Drug. Deliver. Rev. 31, 267-285 (1998).

Online Version 8

[56] T. Naota and H. Koori, "Molecules that assemble by sound: An application to the instant gelation of stable organic fluids," J. Am. Chem. Soc. 127, 9324-9325 (2005). Online Version 8

[57] Schild, "Poly(N-isopropylacrylamide): experiement, theory and application," Prog. Polym. Sci. 17, 163-249 (1992).

Online Version 8, 9, 10

[58] H. Zareiea, E. V. Bulmus, A. Gunning, A. Hoffman, and V. M. E. Piskin, "Investigation of a stimuli-responsive copolymer by atomic force microscopy," Polymer 41, 6723-6727 (2000).

Online Version 8

[59] A. P. Dhanarajan and R. A. Siegel, "Time-dependent permeabilities of hydrophobic, pHsensitive hydrogels exposed to pH gradients," Macromol. Symp. 227, 105-114 (2005). Online Version 10

[60] D. Kuckling, C. Vo, and S. Wohlrab, "Preparation of nanogels with temperatureresponsive core and pH-responsive arms by photo-cross-linking," Langmuir 18, 42634269 (2002).

Online Version 10

[61] M. Ebara, T. Aoyagi, K. Sakai, and T. Okano, "Introducing reactive carboxyl side chains retains phase transition temperature sensitivity in N-isopropylacrylamide copolymer gels," Macromolecules 33, 8312-8316 (2000).

Online Version 10

[62] O. Aoyagi, M. Ebara, K. Sakai, Y. Sakurai, and T. Okano, "Novel bifunctional polymer with reactivity and temperature sensitivity," J. Biomat. Sci. Polym. Ed. 11, 101-110 
(2000).

Online Version 10

[63] M. B. Huglin, Y. Liu, and J. L. Velada, "Thermoreversible swelling behavior of hydrogels based on N-isopropylacrylamide with acidic comonomers," Polymer 38, 5785-5791 (1997).

Online Version 10

[64] M. K. Yoo, Y. K. Sung, Y. M. Lee, and C. S. Cho, "Effect of polyelectrolyte on the lower critical solution temperature of poly(N-isopropyl acrylamide) in the poly(NIPAAm-coacrylic acid) hydrogel," Polymer 41, 5713-5719 (2000).

Online Version 10, 14

[65] J. D. Debord and L. A. Lyon, "Synthesis and characterization of pH-responsive copolymer microgels with tunable volume phase transition temperatures," Langmuir 19, 76627664 (2003).

Online Version 10, 14

[66] N. A. Peppas and A. R. Khare, "Preparation, structure and diffusional behavior of hydrogels in controlled release," Adv. Drug. Deliver. Rev. 11, 1-35 (1993).

Online Version 9

[67] E. S. Gil and S. M. Hudson, "Stimuli-reponsive polymers and their bioconjugates," Prog. Polym. Sci. 29, 1173-1222 (2004).

Online Version 12

[68] A. S. Hoffman, P. S. Stayton, V. Bulmus, G. Chen, and J. Chen, "Really smart bioconjugates of smart polymers and receptor proteins," J. Biomed. Mater. Res. 52, 577-586 (2000).

Online Version 12

[69] E. L. Cussler, M. R. Stokar, and J. E. Varberg, "Gels as size selective extraction solvents," AICHE J. 30, 578-582 (1984).

Online Version 14, 21 
[70] A. E. English, S. Mafé, J. A. Manzanares, X. Yu, A. Y. Grosberg, and T. Tanaka, "Equilibrium swelling properties of polyampholytic hydrogels," J. Chem. Phys. 104, 8713-8720 (1996).

Online Version 14

[71] S. Kang and Y. Bae, "pH-induced volume-phase transition of hydrogels containing sulfonamide side group by reversible crystal formation," Macromolecules 34, 8173-8178 (2001).

\section{Online Version 14}

[72] Y. Nagasaki, E. Honzawa, M. Kato, K. Kataoka, and T. Tsuruta, "Novel stimulisensitive telechelic oligomers. $\mathrm{pH}$ and temperature sensitivities of poly(silamine) oligomers," Macromolecules 27, 4848-4850 (1994).

Online Version 14

[73] E. S. Matsuo and T. Tanaka, "Kinetics of discontinuous volume-phase transition of gels," J. Chem. Phys. 89, 1695-1703 (1988).

Online Version 16, 62, 66, 71

[74] M. Das and E. Kumacheva, "From polyelectrolyte to polyampholyte microgels: comparison of swelling properties," Colloid Polym. Sci. 284, 1073-1084 (2006).

Online Version 16, 18

[75] N. Sahiner, W. T. Godbey, G. L. McPherson, and V. T. John, "Microgel, nanogel and hydrogelhydrogel semi-IPN composites for biomedical applications: synthesis and characterization," Colloid Polym. Sci. 284, 1121-1129 (2006).

Online Version 18

[76] B. G. Kabra and S. H. Gehrke, "Synthesis and characterization of thermally reversible macroporous poly(N-isopropylacrylamide) hydrogels," Polym. Commun. 32, 322-323 (1991). 18, 19

[77] X. S. Wu, A. S. Hoffman, and P. Yager, "Synthesis and characterization of thermally reversible macroporous poly(N-isopropylacrylamide) hydrogels," J. Polym. Sci. 
Pol. Chem. 30, 2121-2129 (1992).

Online Version 18, 19

[78] X.-Z. Zhang and R.-X. Zhuo, "Dynamic properties of temperature-sensitive poly(Nisopropylacrylamide) gel cross-linked through siloxane linkage," Langmuir 17, 12-16 (2001).

Online Version 18

[79] X.-Z. Zhang, Y.-Y. Yang, T.-S. Chung, and K.-X. Ma, "Preparation and characterization of fast response macroporous poly(N-isopropylacrylamide) hydrogels," Langmuir 17, 6094-6099 (2001).

Online Version 18

[80] R.-X. Zhuo and W. Li, "Preparation and characterization of macroporous poly(Nisopropylacrylamide) hydrogels for the controlled release of proteins," J. Polym. Sci. Pol. Chem. 41, 152-159 (2003).

Online Version 18

[81] X.-Z. Zhang and C.-C. Chu, "Fabrication and characterization of microgel-impregnated, thermosensitive PNIPAAm hydrogels," Polymer 46, 9664-9673 (2005).

Online Version 18

[82] H. Omidiana, J. G. Roccaa, and K. Parkb, "Advances in superporous hydrogels," J. Control. Release 103, 3-12 (2005).

Online Version 18, 19

[83] X. Li, X.-Z. Zhang, Y.-F. Chu, X.-D. Xu, S.-X. Cheng, R.-X. Zhuo, and Q.-R. Wang, "Fast responsive poly(N-isopropylacrylamide) hydrogels prepared in phenol aqueous solutions," Eur. Polym. J. 42, 2458-2463 (2006).

Online Version 18, 19

[84] M. Kon and A. de Visser, "A poly(HEMA) sponge for restoration of articular cartilage defects," Plast. Reconstr. Surg. 67, 289-293 (1981). 18 
[85] H. Oxley, P. Corkhill, J. Fitton, and B. Tighe, "Macroporous hydrogels for biomedical applications: Methodology and morphology," Biomaterials 14, 1064-1072 (1993). Online Version 18

[86] K. Kabiri, H. Omidian, and M. J. Zohuriaan-Mehr, "Novel approach to highly porous superabsorbent hydrogels: Synergistic effect of porogens on porosity and swelling rate," Polym. Int. 52, 1158-1164 (2003).

Online Version 19

[87] D. Ceylan and O. Okay, "Macroporous polyisobutylene gels: A novel tough organogel with superfast responsivity," Macromolecules 40, 8742-8749 (2007).

Online Version 19

[88] R. Yoshida, K. Uchida, Y. Kaneko, K. Sakai, A. Kikuchi, Y. Sakurai, and T. Okano, "Comb-type grafted hydrogels with rapid deswelling response to temperature changes," Nature 374, 240-242 (1995).

Online Version 19

[89] Y. Kaneko, S. Nakamura, K. Sakai, T. Aoyagi, A. Kikuchi, Y. Sakurai, and T. Okano, "Rapid deswelling response of poly(N-isopropylacrylamide) hydrogels by the formation of water release channels using poly(ethylene oxide) graft chains," Macromolecules 31, 6099-6105 (1998).

Online Version 19

[90] Y. Noguchi, K. Okeyoshi, and R. Yoshida, "Design of surfactant-grafted hydrogels with fast response to temperature," Macromol. Rapid Commun. 26, 1913-1917 (2005). Online Version 19

[91] H. Abe, K. Hayashi, and M. Sato, eds., Data book on mechanical properties of living cells, tissues, and organs (Springer, Tokyo, Japan, 1996). 20

[92] Y. Tanaka, J. P. Gong, and Y. Osada, "Novel hydrogels with excellent mechanical performance," Prog. Polym. Sci. 30, 1-9 (2005).

Online Version 20 
[93] Y. Okumura and K. Ito, "The polyrotaxane gel: A topological gel by figure-of-eight cross-links," Adv. Mater. 13, 485-487 (2001).

Online Version 20

[94] J. P. Gong, Y. Katsuyama, T. Kurokawa, and Y. Osada, "Double network hydrogels with extremely high mechanical strength," Adv. Mater. 15, 1155-1158 (2003).

Online Version 20

[95] K. Haraguchi and T. Takehisa, "Nanocomposite hydrogels: a unique organicinorganic network structure with extraordinary mechanical, optical, and swelling/de-swelling properties," Adv. Mater. 14, 1120-1124 (2002).

Online Version 20

[96] A. Dhanarajan, G. Misra, and R. Siegel, "Autonomous chemomechanical oscillations in a hydrogel/enzyme system driven by glucose," J. Phys. Chem. A 106, 8835-8838 (2002).

Online Version 21

[97] I. Lokuge, X. Wang, and P. Bohn, "Temperature-controlled flow switching in nanocapillary array membranes mediated by poly(N-isopropylacrylamide) polymer brushes grafted by atom transfer radical polymerization," Langmuir 23, 305-311 (2007).

Online Version 21 


\section{Chapter 3}

\section{Flow Through Reactor}

The continuously stirred tank reactor, abbreviated as CSTR, is a very powerful tool in nonlinear dynamics studies $[1,2,3]$. Many oscillatory reactions have been studied using a CSTR. However, vigorous stirring is necessary in a CSTR to provide thorough mixing. This limits its applications in studies where a stationary environment is required. Here, we develop a new reactor that is based on a CSTR configuration, which we call a flow through reactor. It is composed of two chambers, a regular CSTR as the reactor and a secondary chamber that is attached. The secondary chamber receives the reaction mixture of the primary chamber, and provides a laminar flow of the reactant solution. In this chapter, we will discuss the construction and experiments carried out in a CSTR and flow through reactor. In Chapters 4 and 5 , the flow through reactor will be used as an important component in experiments on chemomechanical oscillators and chemical walkers.

\subsection{Construction of the CSTR}

A CSTR requires constant input of the reactants and constant removal of the reaction mixture. The volume of the reactor is maintained constant and the reaction mixture is homogeneous throughout the reaction. Figure 3.1 shows the basic design of a CSTR. A CSTR generally includes one or more inlets for input of the reactants and one outlet to remove the reaction mixture. Mechanical or magnetic stirring is applied in order to maintain homogeneous conditions inside of the reactor. In order to maintain the constant input of the 


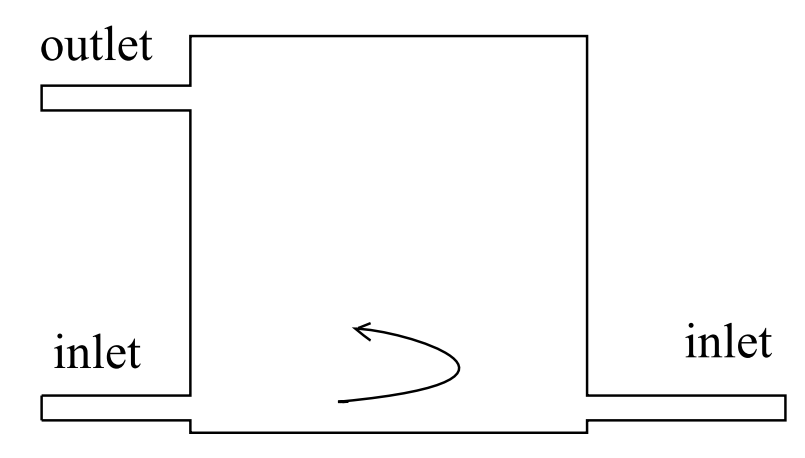

Figure 3.1. Design of a continuously stirred tank reactor. The reactants enter the CSTR through one or more inlets and the products are removed through the outlet. Mechanical or magnetic stirring is required to maintain homogeneous conditions inside of the reactor.

reactants, peristaltic pumps or syringe pumps are used. A general design has the outlet on the top of the reactor so solution is forced out by the entering reactants.

Due to the limited residence time inside a CSTR, the heat exchange between circulating water from a constant temperature water bath and the CSTR must be sufficiently long for thermal equilibrium to be established to avoid temperature gradients. To maintain isothermal conditions, we maximize the heat exchange time by using long coiled tubing for the solution input, and immerse the entire reactor into a water bath.

Another challenge in using the CSTR is maintaining both the homogeneity and vortexfree conditions inside the CSTR. The use of a single high speed magnetic stir bar is generally problematic due to vortex formation. A multi stir bar system was introduced by Swinney's group $[4,5,6]$, which offers both homogeneity and vortex free conditions. We have constructed our reactor according to a similar design (see Appendix B).

To characterize the features of a CSTR, several parameters must be introduced. We denote the volume of the CSTR as $V$, the flow rate of the input and output are the same and equal to $\nu$. We can then calculate the residence time, $\tau$, by using Eq. 3.1. For CSTRs with different volumes, the same $\tau$ value produces the same dynamic behavior if we keep the other parameters constant, such as the concentrations of the reactants and the temperature.

$$
\tau=V / \nu
$$

A number of different dynamical behaviors can be observed in a CSTR. For example, 
bistability, monostability and oscillatory behaviors have been found in the bromate-sulfiteferrocyanide system [3] and ferrocyanide-iodide-sulfite system [1, 2].

\subsection{CSTR Derived Reactors}

As a simple extension of the CSTR, we add another reactor to the CSTR to form a two-

chamber system. We refer to the original CSTR as the primary or main chamber and we refer the added reactor as the secondary chamber. The solution that comes out directly from the primary chamber will enter the second chamber immediately. In order to get repeatable and well controlled conditions, the secondary chamber has to be designed to meet specific requirements. Currently researchers use either inert or chemoresponsive gels as the secondary chamber for the study of patterns and chemical waves $[4,5,7,8]$. In our lab, we use an empty flow-through reactor as the secondary chamber to study chemomechanical oscillators. Two different types of CSTR based systems will be introduced below.

\subsubsection{One-side-fed-reactors}

When polymer or inorganic gels are used as the secondary chamber, the chemicals will diffuse into the gel from one side and convection is removed completely. They are normally called one-side-fed-reactors (OSFRs) [8, 9]. Figure 3.2(a) illustrates one of the simple designs of an OSFR. Conventionally, OSFRs have been designed for the study of patterns and chemical waves $[4,5,7]$; however, recently OSFRs have also been used to study chemomechanical behavior under bistable conditions. For example, a theoretical study by Boissonade [10] showed that when chemoresponsive gels are used as the secondary chamber, a chemomechanical instability may give rise to oscillatory behavior under bistable conditions of the flow system. However, experimental studies have shown traveling waves for these conditions [8].

\subsubsection{Flow Through Reactor}

Besides polymer or inorganic gels, empty flat tubes or circular tubes can also be used as a secondary reactor. Under laminar-flow conditions, diffusion becomes the dominant transport 
solution orthogonal to the flow. Since laminar-flow conditions are required, we must make sure the Reynolds number is sufficiently small in the reactor. The Reynolds number can be calculated by Eq. 3.2 [11].

$$
R_{e}=\frac{\text { inertial forces }}{\text { viscous forces }}=\frac{\rho \nu_{s} L}{\mu}=\frac{\nu_{s} L}{\nu}
$$

where $L$ is the characteristic length in meters, $\nu_{s}$ is the mean fluid velocity in $\mathrm{m} \mathrm{s}^{-1}, \mu$ is the absolute dynamic fluid viscosity in $(\mathrm{Pa}) \mathrm{s}, \nu$ is the kinematic fluid viscosity, defined as $\nu=\mu / \rho$, in $\mathrm{m}^{2} \mathrm{~s}^{-1}$, and $\rho$ is the density of the fluid in $\mathrm{kg} \mathrm{m}^{-3}$.

Two similar reactors have been designed (model CSTR2C1 and model CSTR2C3) and are shown in Fig. 3.2(b), and Fig. 3.2(c). Laminar flow conditions are assumed inside the secondary chamber. For model CSTR2C1, the secondary chamber is tubular shape with the same diameter as the primary chamber and is directly on top of the primary chamber. Between the two chambers is a piece of porous anodisc filter. The flow direction is indicated by the arrows. If there is any difference of the concentrations due to the residence time, inhomogeneity may arise in the vertical direction. For model CSTR2C3, the flow is in the horizontal direction, and inhomogeneity may also arise along this direction.

\subsection{The Bromate-Sulfite-Ferrocyanide System in a Flow Through Reactor}

If diffusion is the dominant transport and the residence time in the secondary chamber is small, the reaction mixture of the secondary chamber will resemble the reaction mixture of the primary chamber. For example, if the primary chamber exhibits oscillations, the secondary chamber should exhibit oscillations as well. Following this idea, the two designs, model CSTR2C1 and CSTR2C3, were tested with the bromate-sulfite-ferrocyanide (BFS) system [3] and the experimental behaviors are described below. 


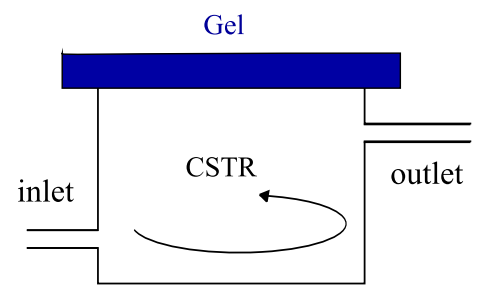

(a)

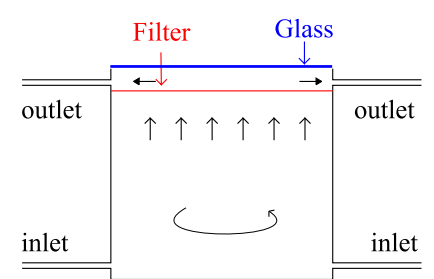

(b)

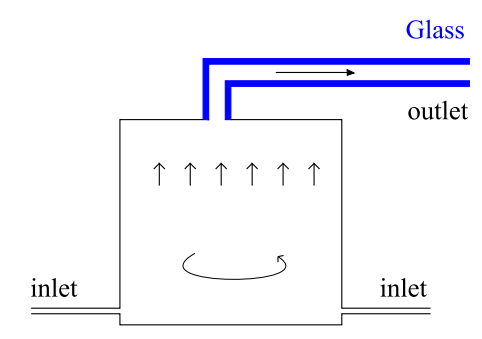

(c)

Figure 3.2. Reactors derived from a CSTR. The dimensions of the reactors are listed in the

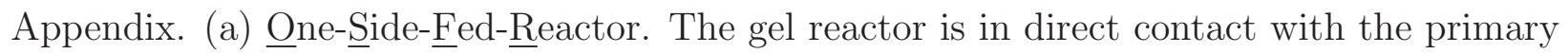
reactor and being constantly fed the reaction mixture from one side. (b) Flow through reactor (Model CSTR2C1). The primary chamber is a standard CSTR, while the secondary chamber is on top of the primary chamber. The primary chamber and secondary chamber are separated by an anodisc filter. The secondary chamber is covered by the circular glass on top to form a closed chamber. (c) Flow through reactor (Model CSTR2C3). The primary chamber is a standard CSTR and the secondary chamber is directly connected to it. The shape of the secondary chamber is rectangular and the flow is indicated by the arrows in the figure. 


\subsubsection{Bromate-Sulfite-Ferrocyanide System}

Experimental Conditions. The thermally regulated CSTR reactor model CSTR2C1 was used, and the temperature was maintained at $22.5 \pm 0.2^{\circ} \mathrm{C}$. Reactant solutions were pumped into the reactor through two inlet tubes by means of a Gilson Minipuls 3 peristaltic pump. The reservoir concentrations were kept constant and the inlet flow rate was varied during the experiments. One reservoir contained $0.130 \mathrm{M}$ sodium bromate and $0.020 \mathrm{M}$ sulfuric acid, while the other contained $0.150 \mathrm{M}$ sodium sulfite and $0.040 \mathrm{M}$ potassium ferrocyanide. The solutions were freshly prepared for each experiment.

Analytical grade $\mathrm{NaBrO}_{3}, \mathrm{KFe}(\mathrm{CN})_{4} \cdot 3 \mathrm{H}_{2} \mathrm{O}$, and $\mathrm{Na}_{2} \mathrm{SO}_{3}$ were purchased from Fisher and used without further purification. The solutions were prepared by dissolving appropriate amount of chemicals in deionized water. The $\mathrm{H}_{2} \mathrm{SO}_{4}$ solution was prepared by diluting concentrated $(18 \mathrm{M}) \mathrm{H}_{2} \mathrm{SO}_{4}$, and the concentration was determined by titration against standard $\mathrm{NaOH}$ solution. The dynamical behavior was measured by the $\mathrm{pH}$ of the solution and greyscale measurements of images taken by a digital camera with a narrow bandpass filter at $425 \mathrm{~nm}$.

Experimental Results. For the bromate-sulfite-ferrocyanide reaction, oscillatory, bistable, and monostable regions exist depending on the input concentrations and the residence times [3]. During the experiments, the concentrations of bromate, sulfite and ferrocyanide were fixed, the residence time was varied by changing the flow rate. Three regions of behavior were found for the primary CSTR chamber. Region I appears when the residence time is larger than $9.2 \mathrm{~min}$. The solution $\mathrm{pH}$ is similar to the $\mathrm{pH}$ at thermodynamic equilibrium, which is close to 3.0 (steady state II). Region II exists when the residence time is between 9.2 and 34.0 min., in which oscillatory behavior was observed with the $\mathrm{pH}$ changing between 3.0 and 7.0. Region III exists for residence time less than $9.2 \mathrm{~min}$. The $\mathrm{pH}$ ranges are between 5.5 to 7.0 depending on the residence time (steady state I). Shorter residence times give rise to higher $\mathrm{pH}$. The phase diagram is depicted in Fig. 3.3, which is in good agreement with the findings of Edblom et al. [3]. 


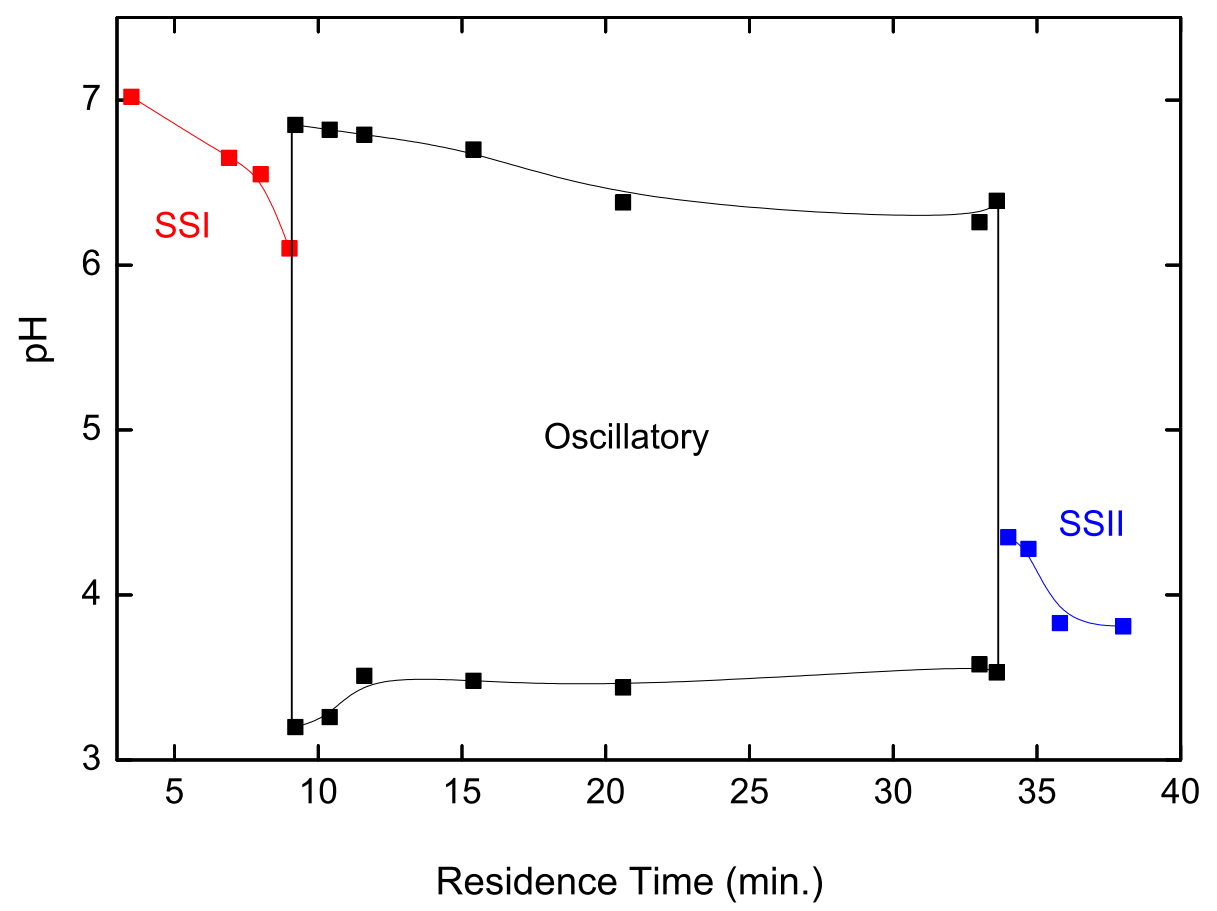

Figure 3.3. Bifurcation diagram for bromate-sulfite-ferrocyanide reaction. The concentrations are $\left[\mathrm{Na}_{2} \mathrm{SO}_{3}\right]=0.075 \mathrm{M},\left[\mathrm{NaBrO}_{3}\right]=0.065 \mathrm{M},\left[\mathrm{K}_{2} \mathrm{Fe}(\mathrm{CN})_{6}\right]=0.020 \mathrm{M}$ and $\left[\mathrm{H}_{2} \mathrm{SO}_{4}\right]$ $=0.010 \mathrm{M}$. The volume of the CSTR is $29.2 \mathrm{ml}$, and the temperature is $22.5^{\circ} \mathrm{C}$.

\subsubsection{Behavior of Bromate-Sulfite-Ferrocyanide System in Reac- tor CSTR2C1}

For model CSTR2C1, the chemical composition in the secondary chamber should be similar to that in the primary chamber when the volume of the secondary chamber is much smaller than the primary chamber. The experimental observations are summarized in Table 3.1 and the typical behaviors are listed in Fig. 3.4.

Experimental results for the bromate-sulfite-ferrocyanide reaction agree well with this expectation when residence times are longer than $34.0 \mathrm{~min}$. Both primary and secondary chambers exhibit steady state II and the $\mathrm{pH}$ values are low. The chemical composition is close to that at thermodynamic equilibrium and the reactants are almost completely consumed. Qualitative agreement with Fig. 3.3 was observed for the dual reactor for residence times 
Table 3.1. Summary of the behaviors for flow through reactor (Model CSTR2C1). The concentrations are $\left[\mathrm{Na}_{2} \mathrm{SO}_{3}\right]=0.075 \mathrm{M},\left[\mathrm{NaBrO}_{3}\right]=0.065 \mathrm{M},\left[\mathrm{K}_{2} \mathrm{Fe}(\mathrm{CN})_{6}\right]=0.020 \mathrm{M}$ and $\left[\mathrm{H}_{2} \mathrm{SO}_{4}\right]=0.010 \mathrm{M}$. Primary and secondary chamber volumes are $31.0 \mathrm{ml}$ and $2.7 \mathrm{ml}$ respectively.

\begin{tabular}{|c|c|c|c|c|}
\hline Region & 1 & 2 & 3 & 4 \\
\hline $\begin{array}{c}\text { Residence time of } \\
\text { primary chamber (min.) }\end{array}$ & $>33.6$ & $33.6-9.2$ & $9.2-4.0$ & $<4.0$ \\
\hline $\begin{array}{c}\text { pH in } \\
\text { primary chamber }\end{array}$ & low pH & oscillatory & high pH & high pH \\
\hline pH in & low pH & oscillatory & low pH & high pH \\
\hline secondary chamber & & & & \\
\hline Typical behavior & Fig. 3.4 (1) & Fig. 3.4 (2), (3), (4) & Fig. 3.4 (5) & Fig. 3.4 (6) \\
\hline
\end{tabular}

between 34.0 and $9.4 \mathrm{~min}$. Both chambers displayed oscillations and the period for the two chambers were the same. However, within each oscillation period, the time at which the oscillator stays at low $\mathrm{pH}$ is longer than that for the secondary chamber. The amplitude is also smaller in the secondary chamber. Surprisingly at short residence times, the $\mathrm{pH}$ in the secondary chamber is very different from that in the primary chamber. Three states were found to exist. When the residence time is between 9.2 and $7.2 \mathrm{~min}$., the primary chamber lies in steady state II and the $\mathrm{pH}$ is low. When the residence time is between 7.2 and 4.0, the secondary chamber shows an inhomogeneous spatial distribution of $\mathrm{pH}$. At residence times shorter than $4.0 \mathrm{~min}$., the secondary chamber follows the primary chamber. The chemical composition is then close to the reactant mixture, with almost no reaction occurring within the CSTR.

The behavior of the secondary chamber depends on its volume and the flow rate of the reactant input. Figure 3.5 shows how the volume of the secondary chamber affects the behavior of the BFS system. The smaller the volume of the secondary chamber, the more similarities in behavior in the two chambers. For example, in a design with a $31.0 \mathrm{ml}$ primary chamber and $2.7 \mathrm{ml}$ secondary chamber, oscillations were found in both chambers 

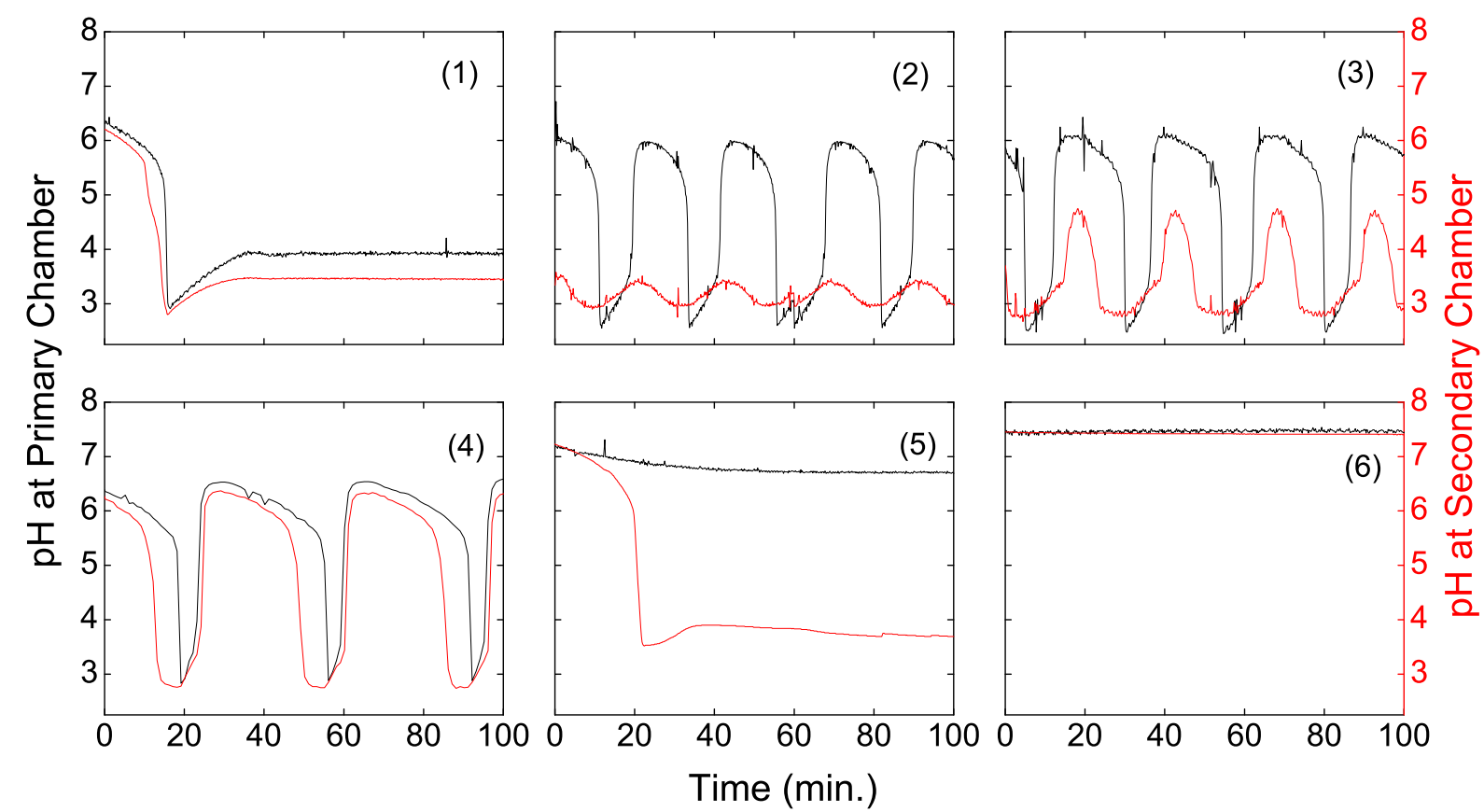

Figure 3.4. Typical behavior of the BFS system in the flow through reactor (CSTR2C1). The black and red lines represent the $\mathrm{pH}$ of the primary and secondary chambers, respectively. The concentrations are $\left[\mathrm{Na}_{2} \mathrm{SO}_{3}\right]=0.075 \mathrm{M},\left[\mathrm{NaBrO}_{3}\right]=0.065 \mathrm{M},\left[\mathrm{K}_{2} \mathrm{Fe}(\mathrm{CN})_{6}\right]=0.020$ $\mathrm{M}$ and $\left[\mathrm{H}_{2} \mathrm{SO}_{4}\right]=0.010 \mathrm{M}$. Primary and secondary chamber volumes are 31.0 and $2.7 \mathrm{ml}$ respectively. Residence time: (1) $\tau=38.0$ min., (2) $\tau=20.6$ min., (3) $\tau=15.4$ min., (4) $\tau$ $=10.4 \mathrm{~min}$. , (5) $\tau=6.9 \mathrm{~min}$. , and (6) $\tau=3.5 \mathrm{~min}$. 


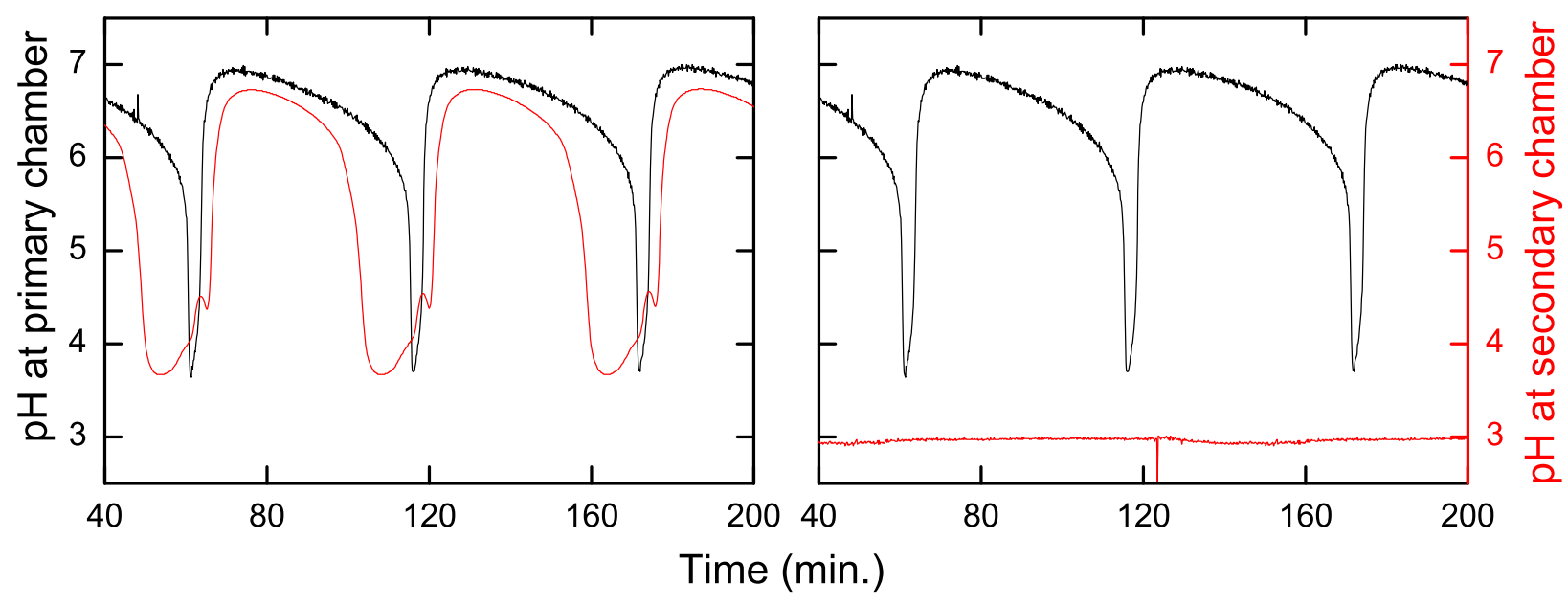

Figure 3.5. Oscillation dependence on the secondary chamber volume for the flow through reactor (CSTR2C1). The black and red lines represent the $\mathrm{pH}$ of the primary and secondary chambers, respectively. Input concentrations: $\left[\mathrm{Na}_{2} \mathrm{SO}_{3}\right]=0.075 \mathrm{M},\left[\mathrm{NaBrO}_{3}\right]=0.065 \mathrm{M}$, $\left[\mathrm{K}_{2} \mathrm{Fe}(\mathrm{CN})_{6}\right]=0.020 \mathrm{M},\left[\mathrm{H}_{2} \mathrm{SO}_{4}\right]=0.010 \mathrm{M} . \tau=10.4 \mathrm{~min}$. Primary chamber volume is 31.0 $\mathrm{ml}$ and secondary chamber volume is (a) $2.7 \mathrm{ml}$ and (b) $5.4 \mathrm{ml}$.

at a flow rate of $2.98 \mathrm{ml} \mathrm{min.}{ }^{-1}$. When the volume of the secondary chamber was doubled, no oscillations were found in the secondary chamber under the same conditions.

Figure 3.4 shows how the flow rate affects the oscillatory behavior of the secondary chamber. In the oscillatory region, a higher flow rate increases the similarities in behavior in the two chambers. For example, at flow rate $6.66 \mathrm{ml} \mathrm{min.} .^{-1}$ and residence time $10.4 \mathrm{~min}$., the $\mathrm{pH}$ oscillation amplitude is almost the same in the two chambers. For flow rate 2.06 $\mathrm{ml} \mathrm{min.} .^{-1}$ and residence time $33.6 \mathrm{~min}$., almost no $\mathrm{pH}$ oscillation were observed. This large discrepancy can be attributed to the extra reaction time in the secondary chamber, since the solution in the secondary chamber is essentially the waste of the CSTR. The solution in the secondary chamber reacts further and will finally go to thermodynamic state if the volume is large. The increase of the secondary chamber volume is equivalent to extending the reaction time. Therefore, the $\mathrm{pH}$ decrease will occur earlier than in the primary chamber and the time the solution stays at low $\mathrm{pH}$ will increase. If the secondary chamber is sufficiently large, the oscillation may completely disappear, as shown in Fig. 3.5. When the residence 
Table 3.2. Summary of the behaviors of the BFS system in the flow through reactor (Model CSTR2C3). The concentrations are $\left[\mathrm{Na}_{2} \mathrm{SO}_{3}\right]=0.075 \mathrm{M},\left[\mathrm{NaBrO}_{3}\right]=0.065 \mathrm{M},\left[\mathrm{K}{ }_{2} \mathrm{Fe}(\mathrm{CN})_{6}\right]$ $=0.020 \mathrm{M}$ and $\left[\mathrm{H}_{2} \mathrm{SO}_{4}\right]=0.010 \mathrm{M}$. The primary chamber volume is $29.2 \mathrm{ml}$, and the secondary chamber volume depends on the length of the chamber (e.g., for a length of 1.5 $\mathrm{cm}$, the volume is $0.30 \mathrm{ml})$.

\begin{tabular}{|c|c|c|c|}
\hline Region & 1 & 2 & 3 \\
\hline $\begin{array}{c}\text { Residence time of } \\
\text { primary chamber (min.) }\end{array}$ & $>33.6$ & $33.6-9.2$ & $<9.2$ \\
\hline pH in & low pH & oscillatory & high pH \\
\hline primary chamber & low pH & Oscillatory & high pH \\
\hline pH in & & & \\
\hline secondary chamber & Fig. 3.6 (1) & Fig. 3.6 (2), (3), (4) & Fig. 3.6 (5), (6) \\
\hline Typical behavior
\end{tabular}

time is decreased (flow rate increased) to below 9.2 min., the primary chamber lies at $\mathrm{pH}$ 5.5, and any extra reaction time will cause a sharp $\mathrm{pH}$ drop in the secondary chamber. A decrease of the secondary chamber volume enables the secondary chamber to follow the primary chamber. However, the volume of the secondary chamber could not be decreased smaller than $1.0 \mathrm{ml}$.

\subsubsection{Behaviors of Bromate-Sulfite-Ferrocyanide System in Reac- tor CSTR2C3}

A new reactor design was introduced in which a flat bed secondary chamber replaced the circular reactor (model CSTR2C3). The height and width of the secondary chamber is fixed and can be precisely controlled for model CSTR2C3. The length determines the total volume of the chamber and is adjustable. The behavior of the BSF system in the CSTR and the secondary flow-through reactor are listed in Table 3.2.

Compared to model CSTR2C1, the volume of the secondary chamber is reduced by 


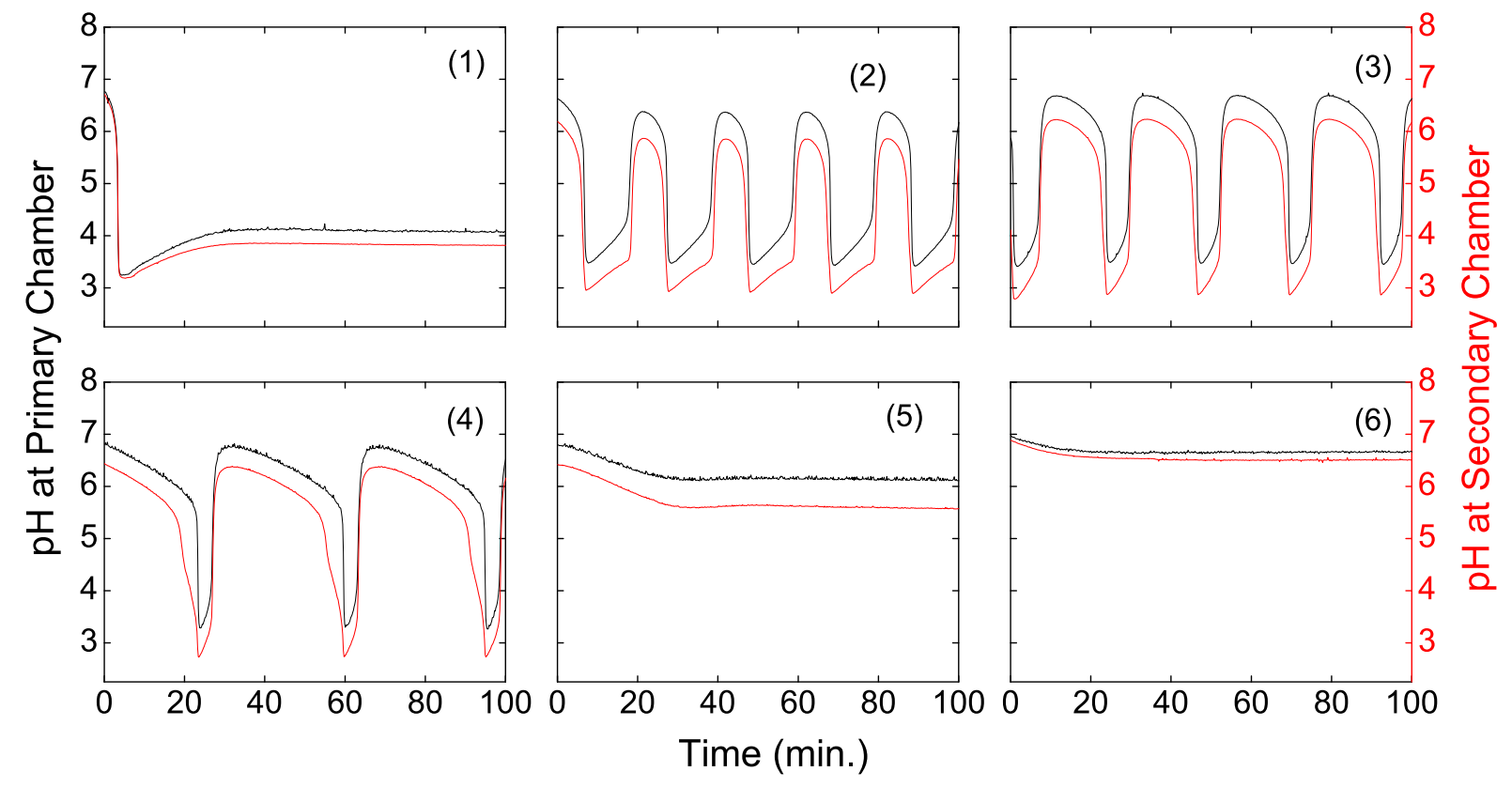

Figure 3.6. Typical behavior of the BFS system in the flow through reactor (CSTR2C3). The black and red lines represent the $\mathrm{pH}$ of the primary and secondary chambers, respectively. The concentrations are $\left[\mathrm{Na}_{2} \mathrm{SO}_{3}\right]=0.075 \mathrm{M},\left[\mathrm{NaBrO}_{3}\right]=0.065 \mathrm{M},\left[\mathrm{K}_{2} \mathrm{Fe}(\mathrm{CN})_{6}\right]=0.020$ $\mathrm{M}$ and $\left[\mathrm{H}_{2} \mathrm{SO}_{4}\right]=0.010 \mathrm{mM}$. The primary chamber volume is $29.2 \mathrm{ml}$, and the secondary chamber volume depends on the length of the chamber (e.g. for a length of $1.5 \mathrm{~cm}$, the volume is $0.30 \mathrm{ml}$ ). The residence time in the CSTR is (1) $\tau=38.0 \mathrm{~min} .,(2) \tau=20.6 \mathrm{~min}$., (3) $\tau=15.4$ min., (4) $\tau=10.4$ min., (5) $\tau=6.9$ min., and (6) $\tau=3.5 \mathrm{~min}$. 
approximately ten fold. The chemical composition is almost the same for both chambers according to $\mathrm{pH}$ measurements, and the dynamic behavior in the CSTR is duplicated in the secondary chamber. At residence times longer than $34.0 \mathrm{~min}$., both chambers lie at steady state II and the $\mathrm{pH}$ is low. At residence times between 34.0 and $9.2 \mathrm{~min}$., both chambers display oscillations between $\mathrm{pH} 3.0$ and 7.0. Both the oscillation period and the time distribution of high and low $\mathrm{pH}$ within a period are the same in both chambers. At a residence time shorter than $9.2 \mathrm{~min}$., both chambers lie at steady state I and the $\mathrm{pH}$ is high. The typical behaviors are shown in Fig. 3.6.

\subsubsection{Spatial Homogeneity of the Secondary Chamber for the Re- actor Model CSTR2C3}

As discussed earlier in this chapter, inhomogeneity may arise in the secondary chamber due to different residence times along the flow direction. In order to analyze the spatial homogeneity of the secondary chamber, simultaneous multiple point monitoring is required. Optical monitoring was used in this work. Although $\mathrm{pH}$ indicators, such as boromothymol blue, should enhance the contrast between the high $\mathrm{pH}$ and low $\mathrm{pH}$ regions, studies carried out by Lee and Swinney [4] showed that optical measurements at $420 \mathrm{~nm}$ produced the same quality of detection. Ferricyanide is the only species that absorbs light at $420 \mathrm{~nm}$ in the BFS system, and the photospectral properties measured by UV-Vis spectroscopy are shown in Fig. 3.7. From Lambert-Beer's law, the absorbance is proportional to the concentration of ferricyanide, and the greyscale value can be used to determine the spatial homogeneity of ferricyanide concentrations.

Experimental images were taken at fixed intervals of the secondary chamber. Random locations in the image were selected and the greyscale values at each sampling point were plotted as a function of time. An additional analysis was carried out by plotting the greyscale value difference between the sampling points to account for the background differences. The results are shown in Fig. 3.8. Figure 3.8(a) shows an image taken during an experiment, where the crosses mark the sampling points for the spatial homogeneity analysis. Figure 3.8(b) shows the oscillations observed for the sampling points. Greyscale value differences, 


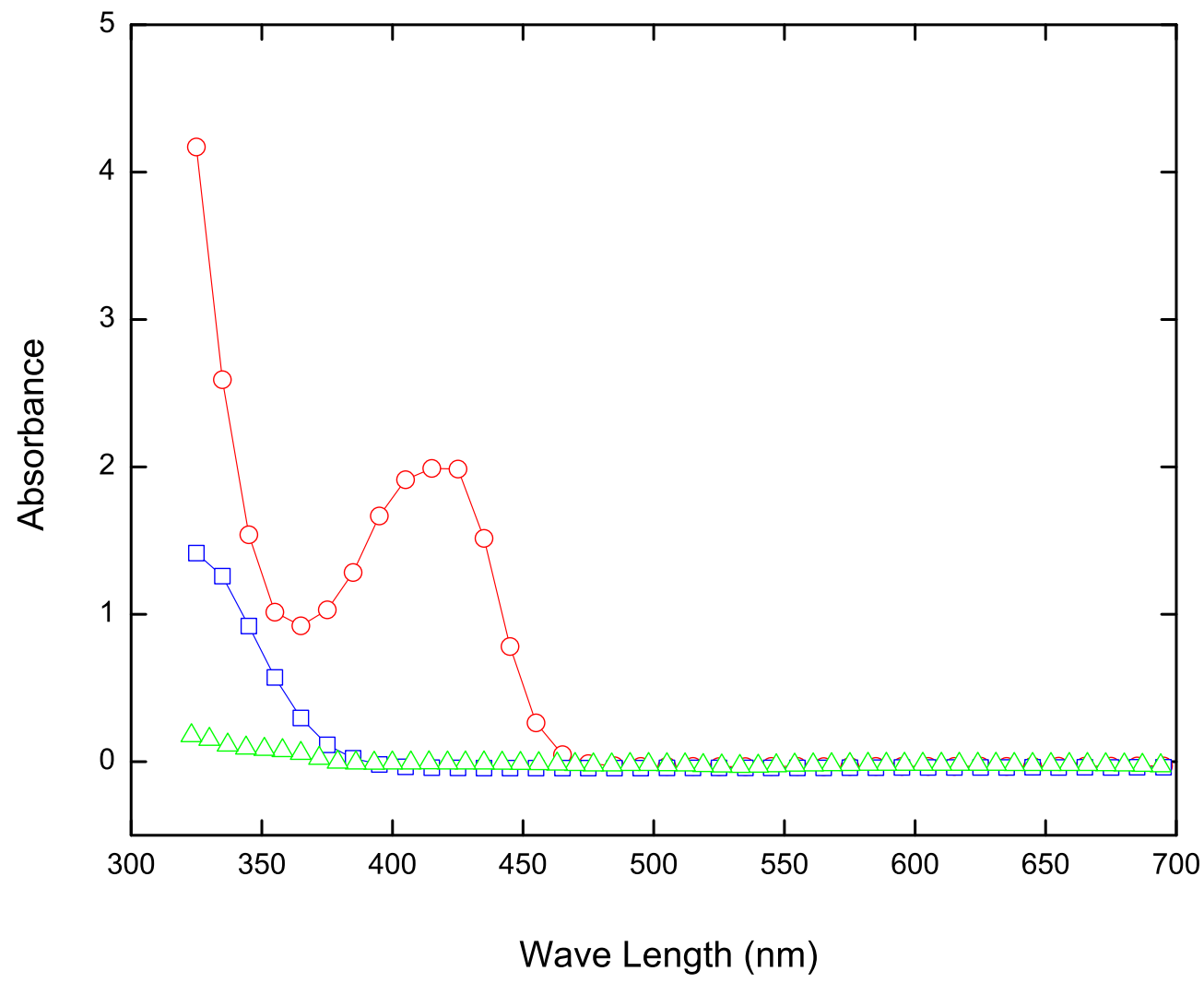

Figure 3.7. Optical absorbance as a function of wavelength for the reactants in the bromatesulfite-ferrocyanide reaction. The red line is for $4.0 \mathrm{mM} \mathrm{K}{ }_{3} \mathrm{Fe}(\mathrm{CN})_{6}$ solution. The blue line is for $4.0 \mathrm{mM} \mathrm{K}_{4} \mathrm{Fe}(\mathrm{CN})_{6}$ solution. The green line is for $0.130 \mathrm{M} \mathrm{NaBrO}_{3}$ and $0.020 \mathrm{M}$ $\mathrm{H}_{2} \mathrm{SO}_{4}$.

which correspond to the concentration differences between different points are constant during the experiment. This indicates the concentration of the ferricyanide is spatially homogeneous and that the laminar flow assumption is valid.

As a comparison, control experiments were also carried out with deionized water as the input to the reactor, as shown in Fig. 3.9. Similarly, the sampling points were randomly selected within the secondary chamber and a greyscale analysis of the sampling points was carried out. 


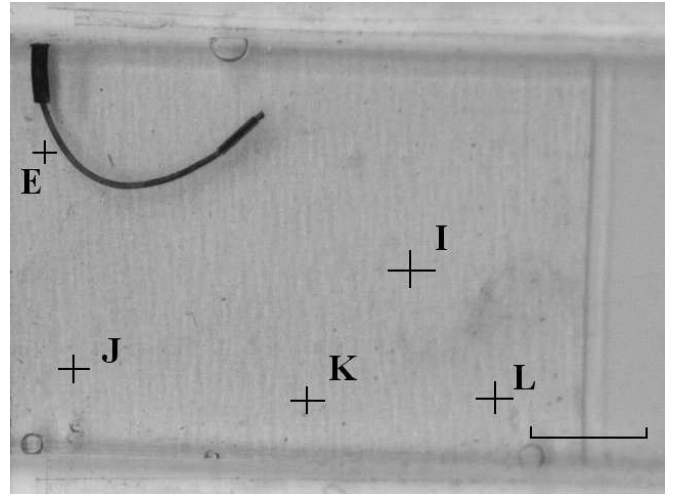

(a)

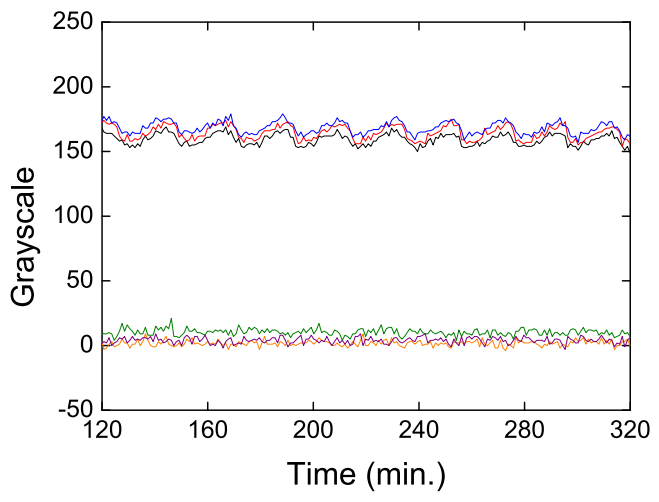

(b)

Figure 3.8. Spatial homogeneity analysis of the flow through reactor model CSTR2C3 for the bromate-sulfite-ferrocyanide reaction. Images were taken from the top of the secondary chamber. The concentrations are $\left[\mathrm{Na}_{2} \mathrm{SO}_{3}\right]=0.075 \mathrm{M},\left[\mathrm{NaBrO}_{3}\right]=0.065 \mathrm{M},\left[\mathrm{K}_{2} \mathrm{Fe}(\mathrm{CN})_{6}\right]=$ $0.020 \mathrm{M}$ and $\left[\mathrm{H}_{2} \mathrm{SO}_{4}\right]=0.010 \mathrm{M}$. The primary chamber volume is $29.2 \mathrm{ml}$, and the residence time $\tau=10.4 \mathrm{~min}$. (a) Sampling points for spatial homogeneity examination by greyscale analysis, where the crosses indicate the sampling positions. The scale bar in the image is $5.0 \mathrm{~mm}$. (b) Greyscale time plot for each point in panel (a) and time plot for greyscale difference between two points. For clarity, not all points in panel (a) are plotted. Black, red and blue lines represent greyscale values for points L, J and I, respectively. Orange, olive and purple lines represent greyscale value differences $G(L)-G(K), G(L)-G(E)$, and $G(I)-G(J)$, respectively. 


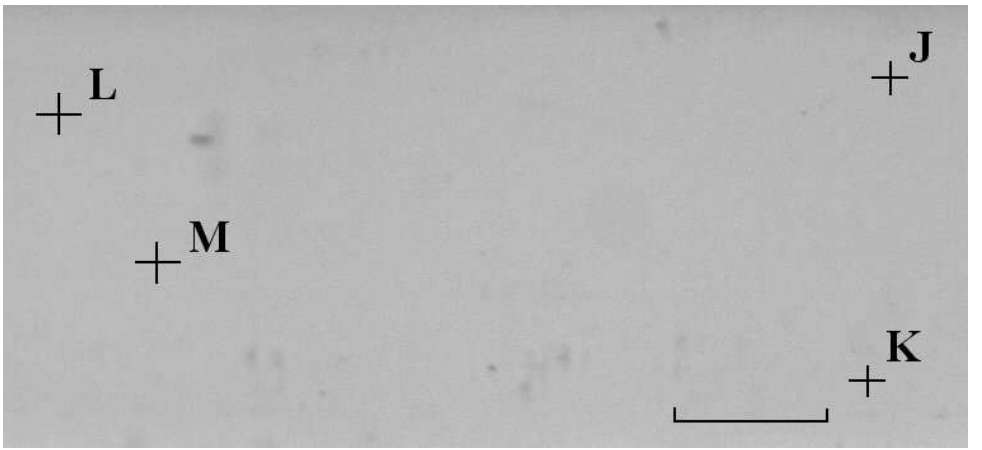

(a)

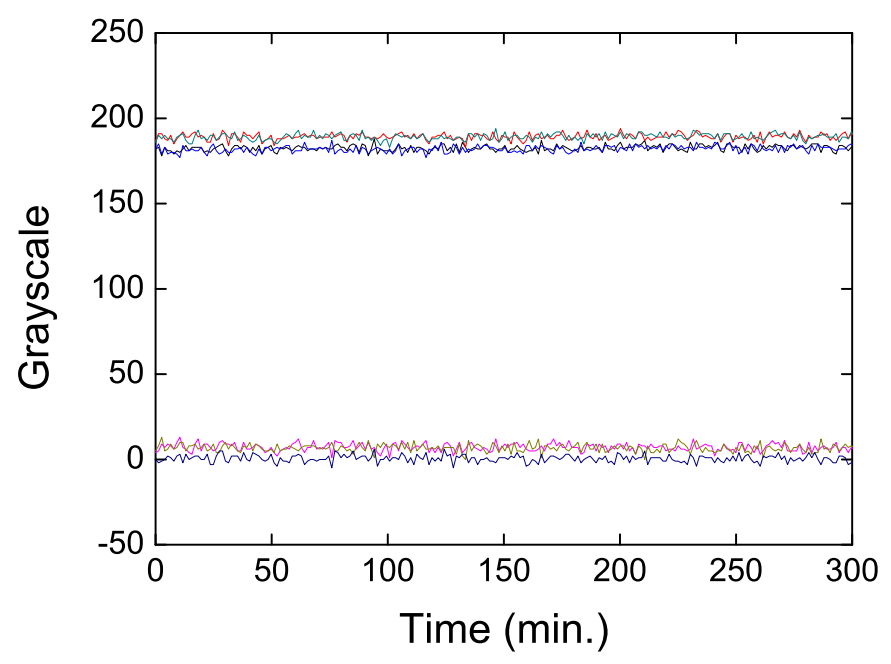

(b)

Figure 3.9. Spatial homogeneity analysis of the flow through reactor model CSTR2C3 for the control. Images were taken from the top of the secondary chamber. The input is deionized water. The primary chamber volume is $29.2 \mathrm{ml}$ and residence time $\tau=10.4 \mathrm{~min}$. (a) Sampling points for spatial homogeneity examination by greyscale analysis, where the crosses indicate the sampling positions. The scale bar in the image is $5.0 \mathrm{~mm}$. (b) Greyscale time plot for each point in panel (a) and time plot for greyscale difference between two points. For clarity, not all points in panel (a) are plotted. Black, red and blue lines represent greyscale values for points L, J and I, respectively. Orange, olive and purple lines represent greyscale value differences $\mathrm{G}(\mathrm{L})-\mathrm{G}(\mathrm{K}), \mathrm{G}(\mathrm{L})-\mathrm{G}(\mathrm{E})$, and $\mathrm{G}(\mathrm{I})-\mathrm{G}(\mathrm{J})$, respectively. 
For the flow through reactor, the volume of the secondary chamber and residence time can be calculated by Eq. 2.3 and Eq. 3.4, respectively. Because the volume is very small, the residence time is negligible compared to the reaction time, which is around $10 \mathrm{~min}$. The concentration is therefore expected to be the same as that in the primary chamber.

$$
\begin{aligned}
\text { volume } & =\text { length } \times \text { width } \times \text { height } \\
& =0.10 \mathrm{~mm} \times 2.00 \mathrm{~cm} \times 2.00 \mathrm{~cm} \\
& =0.40 \mathrm{ml}
\end{aligned}
$$

$$
\begin{aligned}
\text { residence time } & =\frac{\text { volume }}{\text { flow rate }} \\
& =\frac{0.40 \mathrm{ml}}{(31.0 \mathrm{ml} / 10.4 \text { minute })} \\
& =0.13 \text { minute }=8.1 \text { seconds }
\end{aligned}
$$

\subsubsection{Conclusion}

We successfully built a flow through reactor. Model CSTR2C3 offers both design flexibility and operation simplicity. The dynamical behavior in the CSTR was duplicated in the secondary chamber. Experiments that previously could only be carried out in a stationary batch reactor can be carried out with the flow through reactor, providing a new tool for nonlinear studies. 


\section{Bibliography}

[1] E. C. Edblom, L. Györgyi, M. Orbán, and I. R. Epstein, "Systematic design of chemical oscillators. 40. A mechanism for dynamical behavior in the Landolt reaction with ferrocyanide," J. Am. Chem. Soc. 109, 4876-4880 (1987).

Online Version 35, 37, 56

[2] V. Gáspár and K. Showalter, "The oscillatory Landolt reaction. Empirical rate law model and detailed mechanism," J. Am. Chem. Soc. 109, 4869-4876 (1987). Online Version 35, 37, 56

[3] E. C. Edblom, Y. Luo, M. Orbán, K. Kustin, and I. R. Epstein, "Systematic design of chemical oscillators. 45. Kinetics and mechanism of the oscillatory bromate-sulfiteferrocyanide reaction," J. Phys. Chem. 93, 2722-2727 (1989).

Online Version 35, 37, 38, 40, 56

[4] K. J. Lee and H. L. Swinney, "Lamellar structures and self-replicating spots in a reaction-diffusion system," Phys. Rev. E 51, 1899-1915 (1995).

Online Version 36, 37, 47

[5] D. Haim, G. Li, Q. Ouyang, W. D. McCormick, H. L. Swinney, A. Hagberg, and E. Meron, "Breathing spots in a reaction-diffusion system," Phys. Rev. Lett. 77, 190193 (1996).

Online Version 36, 37

[6] G. Li, Q. Ouyang, and H. L. Swinney, "Transitions in two-dimensional patterns in a ferrocyanide-iodate-sulfite reaction," J. Chem. Phys. 105, 10830-10837 (1996).

Online Version 36 
[7] T. Sakurai, E. Mihaliuk, F. Chirila, and K. Showalter, "Design and control of wave propagation patterns in excitable media," Science 296, 2009-2012 (2002).

Online Version 37

[8] V. Labrot, P. D. Kepper, J. Boissonade, I. Szalai, and F. Gauffre, "Wave patterns driven by chemomechanical instabilities in responsive gels," J. Phys. Chem. B 109, 21476-21480 (2005).

Online Version 37, 55

[9] R. Kapral and K. Showalter, eds., Chemical Waves and Patterns (Kluwer, Dordrecht, The Netherlands, 1995). 37

[10] J. Boissonade, "Self-oscillations in chemoresponsive gels: A theoretical approach," Chaos 15, 023703 (2005).

Online Version 37

[11] J. Happel and H. Brenner, Low Reynolds number hydrodynamics : with special applications to particulate media (Kluwer, Boston, MA, USA, 1983). 38 


\section{Chapter 4}

\section{Chemomechanical Oscillators}

\subsection{Definitions}

Here a chemomechanical oscillator refers to a transducer or device that is driven by chemical reactions and changes shape and/or size periodically in time. Chemomechanical oscillators are currently being realized by using polymer gels as the transducer component.

According to the oscillation mechanism, we can divide chemomechanical oscillators into three categories, type I, type II and type III, respectively. For a type I oscillator, the gel which serves as the transducer is completely slaved to the chemical reaction. That is, the chemical reaction is oscillatory both inside and outside of the gel. The oscillator in this work is a type I oscillator. Yoshida et al. [1] and Crook et al. [2] have reported oscillating gels that are also type I oscillators. They are all based on different $\mathrm{pH}$ responsive gels coupled with different pH oscillators. For type II oscillators, the gel contains a catalyst for the oscillatory reaction. The oscillation reaction occurs only inside the gel and the shape or size change does not give feedback to the reaction to a noticeable extent. This is similar to the type I oscillator in that the gel does not affect the chemistry; however, it is very different in that the oscillatory chemical reaction occurs only in the gel while the environment is quiescent. The only example of a type II chemomechanical oscillator was developed by Yoshida and co-workers $[3,4,5,6,7]$. The gel contains a ruthenium complex, which is the catalyst for the Belousov-Zhabotinsky reaction. The gel oscillates in size by following the oxidation state

change of ruthenium between +2 and +3 during the reaction. For Type III chemomechanical 
oscillators, the oscillation occurs inside the gel but also depends on the size or shape change of the gel, which plays a dominant role. De Kepper and co-workers [8] developed a type III oscillator by coupling a $\mathrm{pH}$ responsive gel with a bistable chlorite-tetrathionate (CT) reaction.

\subsection{Construction of a Chemomechanical Oscillator}

A type I chemomechanical oscillator has been developed based on $\mathrm{pH}$ responsive gels and oscillatory $\mathrm{pH}$ reaction. The $\mathrm{pH}$ responsive gel serves as the transducer and it is studied in the secondary chamber of a flow through reactor. The $\mathrm{pH}$ oscillations provide the stimuli to the $\mathrm{pH}$ responsive gel to drive the gel size and shape changes periodically.

\subsubsection{Selection of the $\mathrm{pH}$ Responsive Gel}

There are many different types of $\mathrm{pH}$ responsive gels, as indicated in Chapter 2. An ideal candidate should have a large swelling-deswelling ratio, a sharp pH phase transition, a fast response, and a high mechanical strength. Poly(NIPAAm-co-MAAc) gels meet all the above requirements and hence were chosen for the experiments. According to the original studies of the gel [9], the volume of the swollen gel is up to 16 times larger than the volume of the contracted state. The phase transition of the polymer gel starts from $\mathrm{pH} 5.0$ and ends at pH 6.0 at $40^{\circ} \mathrm{C}$, which is within the range of many available $\mathrm{pH}$ oscillators. The poly(NIPAAmco-MAAc) gels are strong and allow the preparation of small diameter rod shaped gels. Rods with diameters as small as 50 micrometers can be prepared, which exhibit fast response times compared to larger gels.

\subsubsection{Selection of the $\mathrm{pH}$ Oscillator}

The response of the gel to the stimuli is generally slow. In order to have a large volume oscillation, the ideal pH oscillator should have the following characteristics [2]:

1. The $\mathrm{pH}$ oscillator should oscillate over a relatively large $\mathrm{pH}$ range so that it includes the $\mathrm{pH}$ range of the $\mathrm{pH}$ phase transition of the gel. 
2. The oscillator period should be comparable to the equilibrium swelling-deswelling time of the gel, allowing the mechanical oscillation amplitude to be maximized.

3. Reactions that exhibit room temperature $\mathrm{pH}$ oscillators offer experimental convenience.

4. A batch $\mathrm{pH}$ oscillator is superior to an oscillator requiring a CSTR, since the CSTR requires mixing of the reagents.

Among the available pH oscillators $[10,11,12,13,14,15,16,17,18,19]$, there are no examples that operate under batch conditions. Therefore, the oscillatory bromate-sulfiteferrocyanide reaction [13] was selected since it meets all of the above conditions except the last and it is superior to the other $\mathrm{pH}$ oscillators in several aspects. For the oscillatory bromate-sulfite-ferrocyanide reaction, the oscillation ranges from $\mathrm{pH} 3.0$ to $\mathrm{pH} 7.0$ and the phase transition $\mathrm{pH}$ of poly(NIPAAm-co-MAAc) gel, which is close to 5.0, lies in the middle of this range. The oscillation period is longer than $30 \mathrm{~min}$. and the high and low $\mathrm{pH}$ intervals are more than $5 \mathrm{~min}$. It is a room temperature oscillator, although oscillations also occur at higher temperatures. From Chapter 3, we see that the above requirements can be met by using a flow through reactor with the bromate-sulfite-ferrocyanide system.

\subsection{Experimental Setup: Reactor}

Based on the above discussion, the experimental setup was designed as shown in Fig. 4.1. A flow through reactor was used and the $\mathrm{pH}$ responsive gel was placed into the secondary chamber. For better imaging, the bottom side of the secondary chamber was covered with white filter paper. The behavior of the gel was monitored with a Silicon Imaging SI-1280-U CCD camera equipped with a Canon macro lens FD $50 \mathrm{~mm}$ f/3.5. Images were taken and saved on a computer hard drive at fixed intervals. The $\mathrm{pH}$ in both chambers was monitored by pH microelectrodes (MI-4146 and MI-406 from Microelectrodes Inc.), which were connected to a $\mathrm{pH}$ meter (Orion $720 \mathrm{~A}+$ ). Home-made software was used for the image acquisition and the $\mathrm{pH}$ recording. The solutions were pumped into the reactor by Gilson Minipuls 3 peristaltic pumps. The inlet flow rate was varied while the reservoir concentrations were kept constant in all experiments. One reservoir contained $0.130 \mathrm{M}$ sodium bromate and 


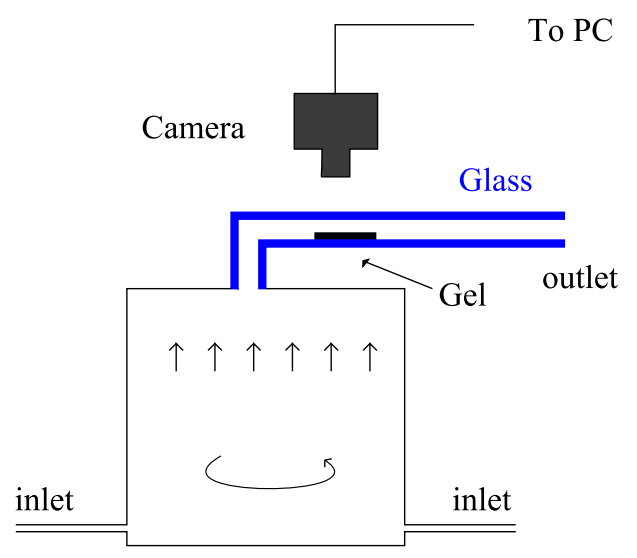

(a)

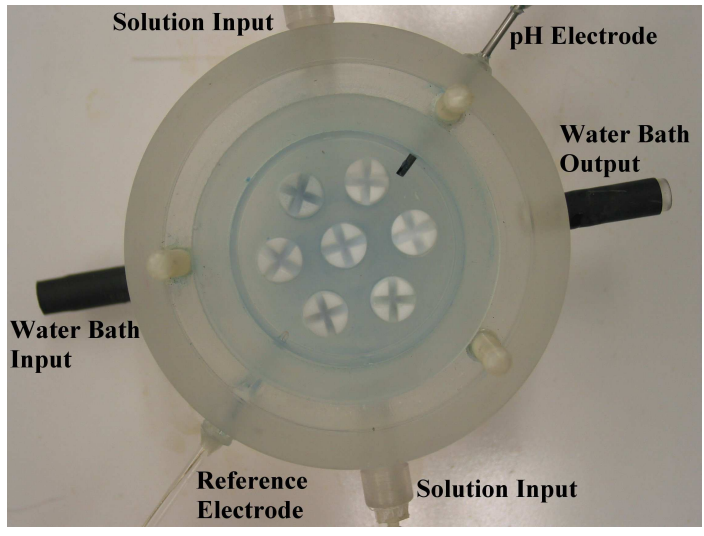

(c)

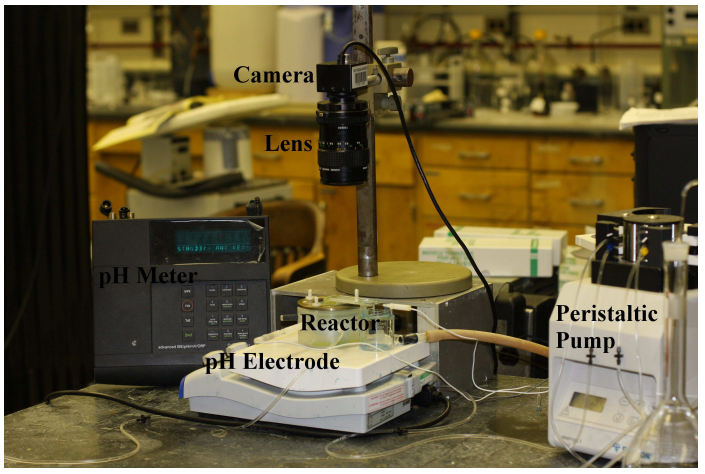

(b)

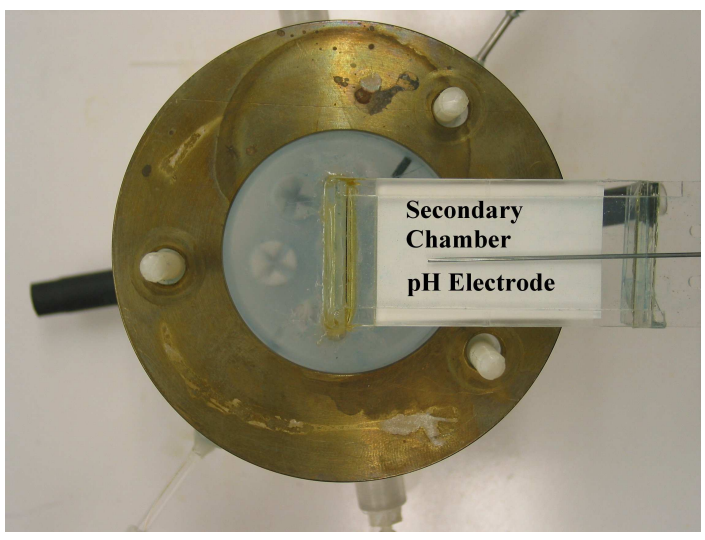

(d)

Figure 4.1. Setup for the chemomechanical oscillator experiment. The volume of the primary chamber is $29.2 \mathrm{ml}$, and the secondary chamber is $1.0 \mathrm{~mm}$ in the vertical direction and 20.0 $\mathrm{mm}$ wide. The gel is placed into the secondary chamber, where the flow distance between the gel and the primary chamber is between $5.0 \mathrm{~mm}$ and $25.0 \mathrm{~mm}$. (a) Sketch of the experimental setup, and photographs of (b) experimental setup, (c) primary chamber, and (d) secondary chamber. 
$0.020 \mathrm{M}$ sulfuric acid. The other contained $0.150 \mathrm{M}$ sodium sulfite and $0.040 \mathrm{M}$ potassium ferrocyanide. Freshly prepared solutions were used for each experiment.

\subsection{Experimental Setup: Properties of the poly(NIPAAm- co-MAAc) Gel}

\subsubsection{Synthesis of the poly(NIPAAm-co-MAAc) Gel}

The $\mathrm{pH}$-sensitive gel we used was the copolymer of NIPAAm and methacrylic acid and it was synthesized according to the Brazel and Peppas method [9]. Briefly, $1.8106 \mathrm{~g}$ NIPAAm, 22.5 mg 2,2'-azobis(isobutyronitrile) (AIBN) , $340 \mu \mathrm{l}$ MAA and $38 \mu$ l ethylene glycol dimethacrylate (EGDMA) were dissolved in $2.4 \mathrm{ml}$ 1:1 mixture of water and methanol. Before polymerization, nitrogen was bubbled through the solution to remove the oxygen, and the solution then filled a capillary by capillary action. After both ends were sealed, the capillary was placed in a water bath and the polymerization was allowed to take place for 24 hours at $50^{\circ} \mathrm{C}$. After polymerization, the gel rods were removed from the capillary immediately or after overnight immersion in $0.72 \mathrm{M}$ sulfuric acid.

\subsubsection{Structure of the Gel}

The poly(NIPAAm-co-MAAc) gel contains both temperature responsive and $\mathrm{pH}$ responsive units. The NIPAAm is responsible for the temperature sensitivity and the methacrylic acid is responsible for $\mathrm{pH}$ sensitivity. The postulated structure is shown in Fig. 4.2. The mole ratio between NIPAAm and methacrylic acid varies depending on the recipe according to the Brazel and Peppas studies [9]. The mole ratio used in this work is 3 to 1 . The gel is a random copolymer between NIPAAm and methacrylic acid.

\subsubsection{Visualization of the Gel}

Since the poly(NIPAAm-co-MAAc) gel is transparent, it can not be observed by the camera directly. We used carbon black to visualize the gel by immersing it into a suspension solution 


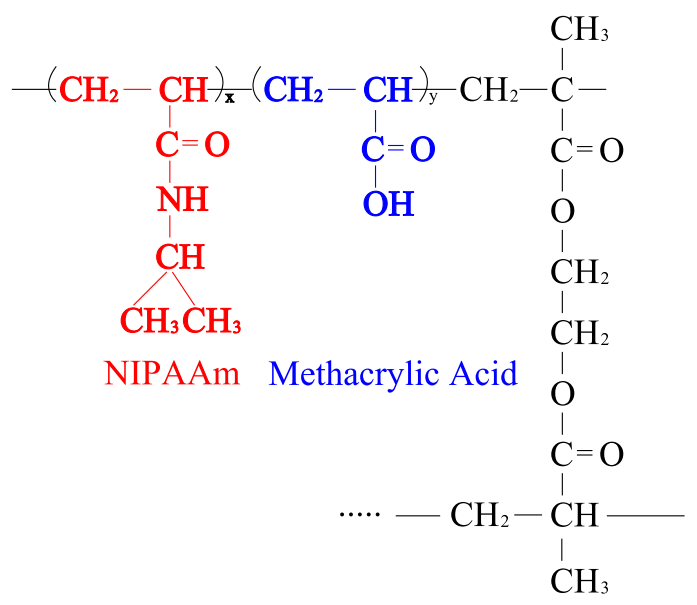

Figure 4.2. Structure of the poly(NIPAAm-co-MAAc) gel. Poly(NIPAAm-co-MAAc) contains 2 functional units, methacrylic acid and $N$-isopropylacrylamide, which are connected by the MBAA. The ratio varies according the prepolymerization ratios. The positions are random. The carboxylic group can be either protonated or deprotonated depending on the environmental $\mathrm{pH}$ and temperature.

of carbon black and agitating for 5 to 10 min. The carbon black was absorbed on the surface of the gel. The carbon black can be washed out with vigorous agitation in pure water.

\subsubsection{Equilibrium Swelling of the Gel}

The poly(NIPAAm-co-MAAc) gel is an acidic gel, as it contains carboxylic acid groups in the network structure. At pH higher than 5.0, the carboxylic acid groups become ionized and the gel swells. The maximum swelling occurs at $\mathrm{pH}$ 6.5, with the volume swelling by 16 times at room temperature. At $\mathrm{pH}$ lower than 5.0, the carboxylic acid groups are protonated and the gel shrinks. The gel swells to different extents according to the percentage of the methacrylic acid. Copolymers that contain $25 \%$ mole methacrylic acid have maximum swelling ratios at $37^{0} \mathrm{C}$ according to Brazel and Peppas [9].

The equilibrium swelling was measured at room temperature. All the $\mathrm{pH}$ buffer solutions except pH 10.0 were prepared according to Table 4.1 [20] (the pH 10.0 buffer was purchased from Fisher and used directly). The maximum swelling was achieved at $\mathrm{pH} 6.0$, with the volume of maximum swelling about 10 times of the volume at $\mathrm{pH}$ 4.0. A sharp phase 
Table 4.1. Table for $\mathrm{pH}$ buffer preparation

\begin{tabular}{|c|c|c|c|}
\hline $\mathrm{pH}$ Required & $0.2 \mathrm{M} \mathrm{Na}_{2} \mathrm{HPO}_{4}(\mathrm{ml})$ & $0.1 \mathrm{M}$ Citric Acid $(\mathrm{ml})$ & $\mathrm{pH}$ \\
\hline 2.2 & 0.40 & 19.60 & 2.20 \\
\hline 3.0 & 4.11 & 15.89 & 3.02 \\
\hline 4.0 & 7.71 & 12.29 & 3.95 \\
\hline 4.6 & 9.35 & 10.65 & 4.53 \\
\hline 5.0 & 10.30 & 9.70 & 4.94 \\
\hline 5.6 & 11.60 & 8.40 & 5.51 \\
\hline 6.0 & 12.63 & 7.37 & 5.97 \\
\hline 7.0 & 16.47 & 3.53 & 6.97 \\
\hline 8.0 & 19.45 & 0.55 & 8.03 \\
\hline
\end{tabular}

transition occurs between $\mathrm{pH} 4.0$ and $\mathrm{pH}$ 5.5. The equilibrium swelling results are shown in Fig. 4.3. Nominal diameter is the diameter of the capillary used to synthesize the gel rod and it is about half of the equilibrium diameter at $\mathrm{pH}$ 7.0.

Our results are not in agreement with those of Brazel and Peppas [9], as the phase transition $\mathrm{pH}$ by our measurements is lower. The phase transition $\mathrm{pH}$ lies between 4.0 and 6.0, compared to 5.0 to 6.5 according to Brazel and Peppas [9]. The disagreement is due to the different measurement temperatures. The gel, poly (NIPAAm-co-MAAc) contains both temperature responsive units and $\mathrm{pH}$ responsive units. At temperatures higher than the phase transition temperature, as in the Brazel and Peppas experiment, NIPAAm shrinks and becomes more hydrophobic. As other studies suggest [21], hydrophilic groups inside the gel network shift the phase transition $\mathrm{pH}$ to a higher $\mathrm{pH}$. The hydrophobicity change for the NIPAAm contributes to the observed differences. 


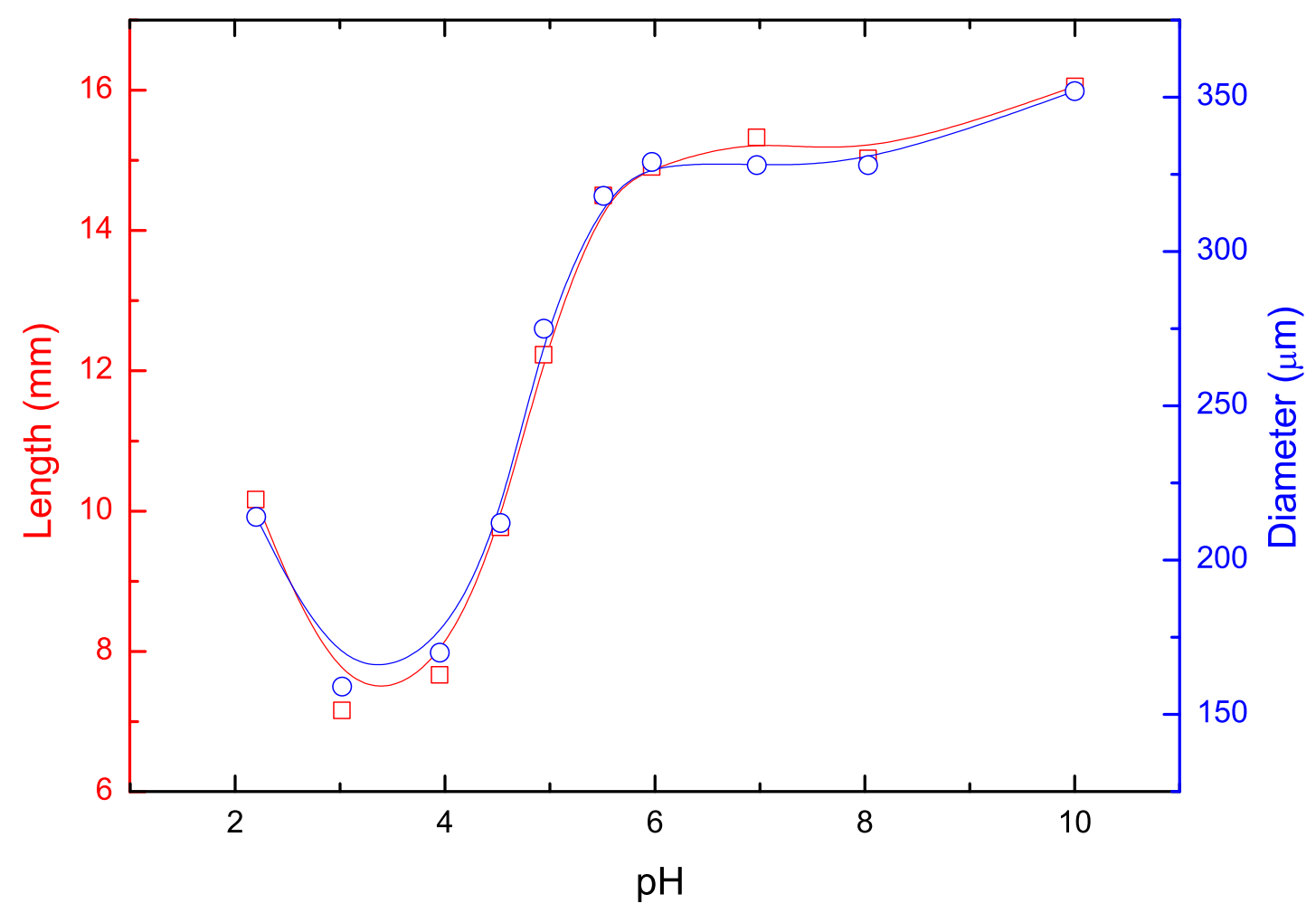

Figure 4.3. Equilibrium length as a function of $\mathrm{pH}$ for the poly(NIPAAm-co-MAAc) gel at room temperature. The red squares represent the equilibrium length of the gel rod and the blue circles represent the equilibrium diameter. The equilibrium length and diameter of the gel rod were measured by a caliper after the gel was immersed into the buffer solution for at least $30 \mathrm{~min}$. The $\mathrm{pH}$ buffer was prepared by mixing the appropriate amounts of $0.2 \mathrm{M}$ $\mathrm{Na}_{2} \mathrm{HPO}_{4}$ and $0.1 \mathrm{M}$ citric acid according to Table 4.1. 


\subsubsection{Dynamic Process of the Gel Swelling and the Swelling Ki- netics}

The poly(NIPAAm-co-MAA) gel is an acidic gel because of the carboxylic groups of the network. The gel swells by absorbing solution into the network when the environment $\mathrm{pH}$ is above the pKa of methacrylic acid. Equilibrium is not established until the internal and external $\mathrm{pH}$ of the gel are the same, and the swelling therefore depends on the rate of mass transport. As introduced in Chapter 2, diffusion is the primary mass transport inside the polymer network. The time required for the swelling is therefore strongly dependent on the length scale of the gel: the smaller the gel, the faster the response.

In order to acquire the swelling kinetic data, experiments were carried out using the following procedure. The gel rod was immersed into a $\mathrm{pH} 4.0$ buffer solution until equilibrium was established. The gel was then transferred to a $\mathrm{pH} 7.0$ buffer solution. Images of the gel were taken at fixed intervals with a camera attached to the microscope. No stirring was applied during the experiments. After the experiments, the diameters of the gel rods were calculated from the images. The length of all the gel rods was at least 5 times larger than their diameter. We tested gel rods with diameters ranging from $493 \mu \mathrm{m}$ to $3587 \mu \mathrm{m}$. A typical swelling curve is shown in Fig. 4.4(a), and the swelling time dependence on gel rod diameter is shown in Fig. 4.4(b).

Figure 4.4 indicates that the swelling is a first order process for gel rods of fixed diameters. This observation is in agreement with the swelling process of spherical poly(NIPAAm) gel for the temperature induced volume phase transitions [22]. Comparing gel rods of different diameters, the square root of the swelling time is proportional to the diameter of the gel rods. The smaller the diameter, the shorter the swelling time.

All sizes of gel rods tested swelled to their respective equilibrium states without forming bumps on the gel surface. Both ends of the gel rods swelled first due to the large contact surface area; then the surface and finally the core started to swell. A typical swelling process is shown in Fig. 4.5. Buckling occurred for all sizes of gel rods because the surface is under pressure to expand and the gel core acts to prevent expansion [22, 23]. The nonhydrolyzed gel core can be clearly seen in Fig. 4.5, which disappears after equilibrium is established. 


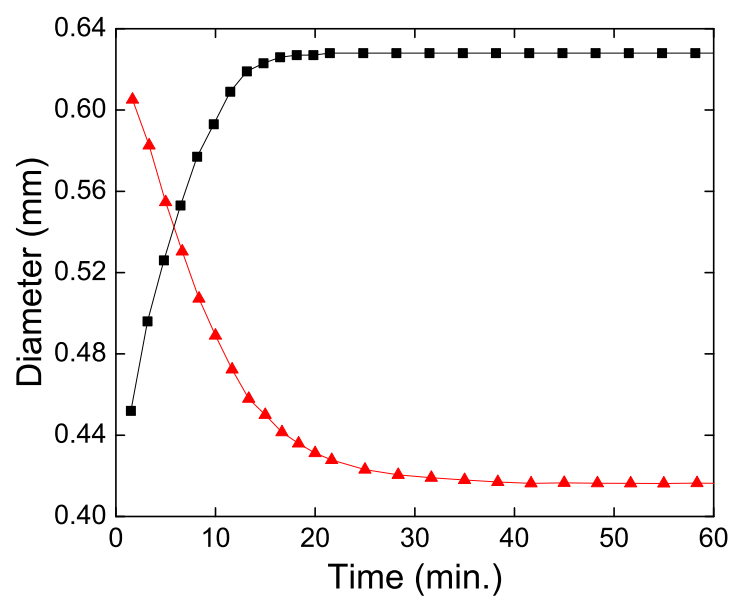

(a)

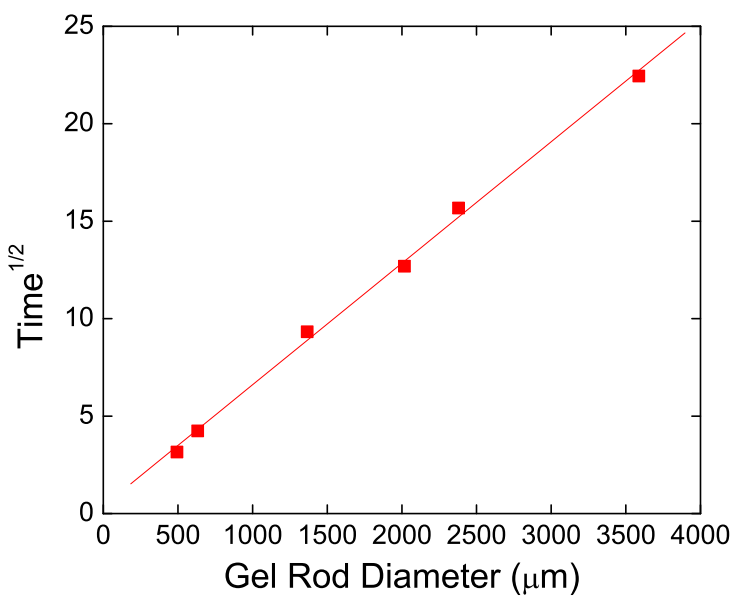

(b)

Figure 4.4. Swelling kinetics at room temperature for poly(NIPAAm-co-MAAc) gel. The gel rod was placed into a $\mathrm{pH} 4.0$ buffer solution until equilibrium was reached. The gel rod was then transferred into $\mathrm{pH} 7.0$ buffer solution. The swelling process was monitored and recorded. A similar procedure was followed for the deswelling process. The gel rod was transferred into $\mathrm{pH} 7.0$ buffer solution after equilibrium was established with $\mathrm{pH} 4.0$ buffer solution. (a) Swelling and deswelling curve of a $632 \mu \mathrm{m}$ diameter gel rod (size at $\mathrm{pH}$ 7.0). (b) Swelling time dependence on gel rod diameter. The red line is a linear fit for the measurements of diameter as a function of $t^{1 / 2}$. 


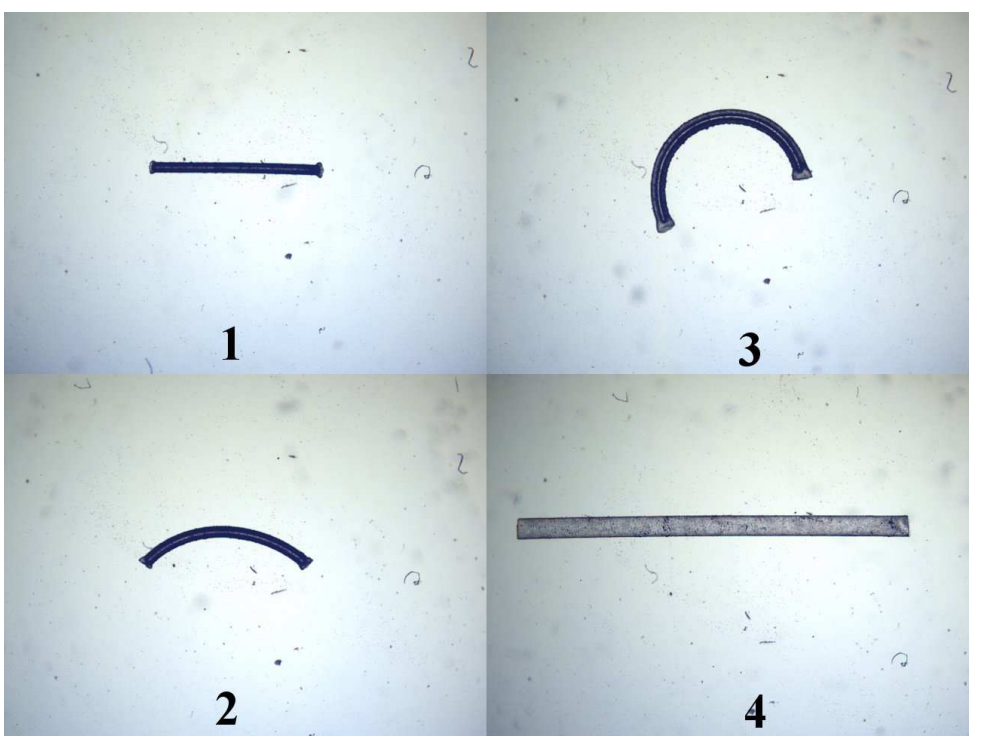

Figure 4.5. Swelling process at room temperature for poly(NIPAAm-co-MAAc) gel. The gel rod $\left(319 \mu \mathrm{m}\right.$ nominal diameter) was placed into $0.72 \mathrm{M} \mathrm{H}_{2} \mathrm{SO}_{4}$ until equilibrium was established. The gel rod was then transferred into $\mathrm{pH} 7.0$ buffer solution. The nominal diameter is the diameter of the capillary used to synthesize the gel. It is about half of the gel diameter at $\mathrm{pH}$ 7.0. The swelling process was monitored and photographically recorded at the time intervals (1) $112 \mathrm{~s}$, (2) $190 \mathrm{~s}$, (3) $420 \mathrm{~s}$, and (4) $850 \mathrm{~s}$. 


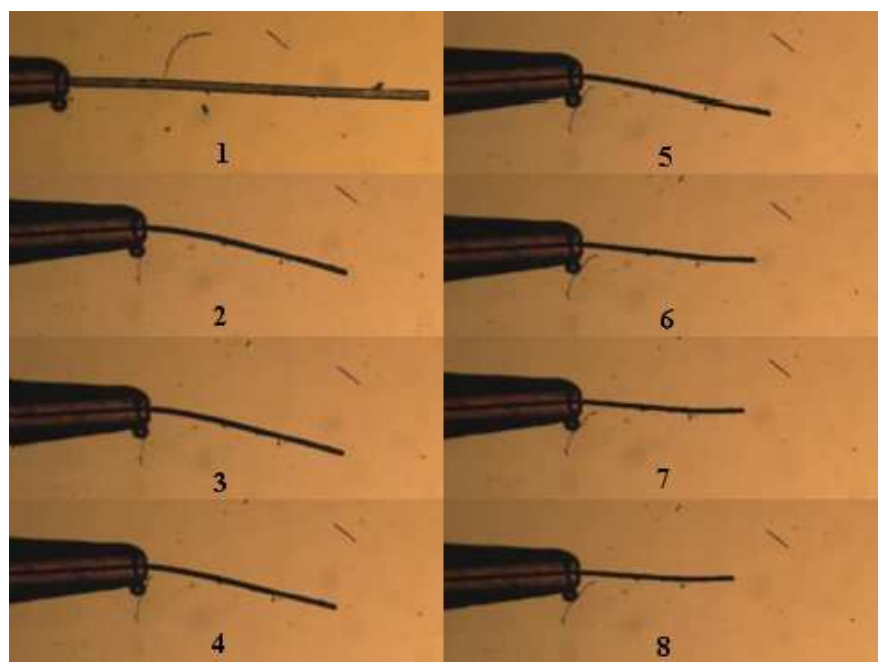

Figure 4.6. Deswelling process of poly(NIPAAm-co-MAAc) gel rod at room temperature (nominal gel rod diameter $100 \mu \mathrm{m}$ ). The gel rod was first equilibrated with $\mathrm{pH} 7.0$ buffer solution and then transferred into $0.72 \mathrm{M} \mathrm{H}_{2} \mathrm{SO}_{4}$. The behavior was recorded as a function of time: (1) 0 min., (2) 1.0 min., (3) 1.2 min., (4) 1.5 min., (5) 2.0 min., (6) 6.0 min., (7) 13.5 min., and (8) 31.0 min.

\subsubsection{Dynamic Process of the Gel Deswelling and the Deswelling Kinetics}

The deswelling depends on mass transport similar to the swelling process. The deswelling experiments were similar to the swelling experiments. The poly(NIPA-co-MAA) gel rods were immersed into $\mathrm{pH} 7.0$ buffer solutions until equilibrium was established. The volume of the $\mathrm{pH}$ buffer solutions was always more than 100 times the volume of the gel rods. The gel rod was then transferred into large amounts of $\mathrm{pH} 4.0$ buffer. Images were taken with the CCD camera at fixed intervals.

The deswelling process of the gel rods is size dependent. For gel rods with nominal diameters less than $200 \mu \mathrm{m}$, the deswelling is relatively fast. The gel rods buckled initially and then became straight. Longer gel rods sometimes initially coiled and then slowly decoiled. The gel surface is smooth throughout the deswelling process. A typical deswelling process is shown in Fig. 4.6.

For gel rods with nominal diameters larger than $200 \mu \mathrm{m}$, the gels buckle first and require 


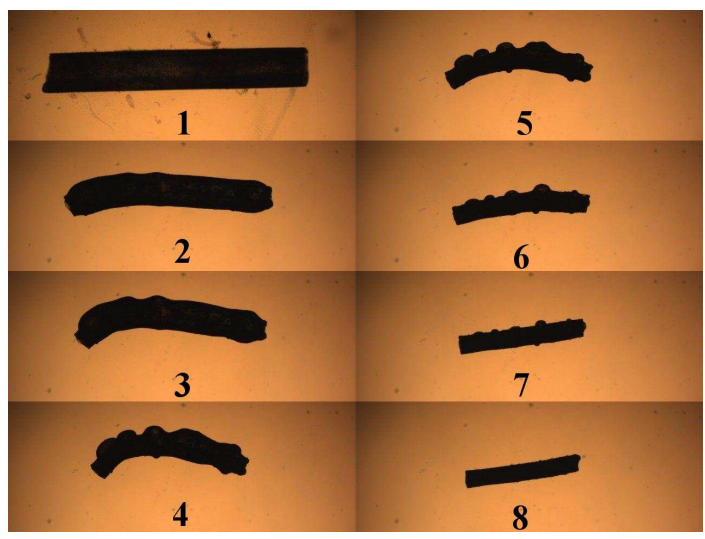

Figure 4.7. Deswelling process of poly(NIPAAm-co-MAAc) gel rod at room temperature (nominal gel rod diameter $537 \mu \mathrm{m}$ ). The gel rod was equilibrated with pH 7.0 buffer solution first and then transferred into $0.72 \mathrm{M} \mathrm{H}_{2} \mathrm{SO}_{4}$. The behavior was recorded as a function of time: (1) 0 min., (2) 17.0 min., (3) 29.0 min., (4) 53.0 min., (5) 65.0 min., (6) 80.0 min., (7) $116.0 \mathrm{~min}$. , and (8) $430.0 \mathrm{~min}$.

much longer times to become straight. Round bumps form on the surface of the gel during the deswelling process. A typical deswelling process is shown in Fig. 4.7.

The deswelling process of the gels also depended on the buffer solutions used. If the deswelling is carried out stepwise by soaking the gels in a series of $\mathrm{pH}$ buffers, significant buckling may not be observed, as shown in Fig. 4.8.

The drying of a gel in air is quite similar to the deswelling of a gel in pH buffer solutions. In both cases water is expelled out of the polymer network. However, the deswelling of the $\mathrm{pH}$ responsive gels is better controlled than the drying process. Air drying generally does not shrink the gel uniformly in each direction, and uniform gel rods can not be acquired by drying a gel in air. The buckling of the gel rod will be explained in Section 4.5.2.

Compared to the swelling process, the deswelling process requires significantly longer times to reach equilibrium. At the beginning of the deswelling, however, the rate of the size changes is comparable to that of the swelling. This observation is in agreement with the deswelling process of spherical poly(NIPAAm) gels during temperature induced volume phase transitions [22].

When the poly(NIPAAm-co-MAA) gel rods are switched from low $\mathrm{pH}$ to high $\mathrm{pH}$, the 


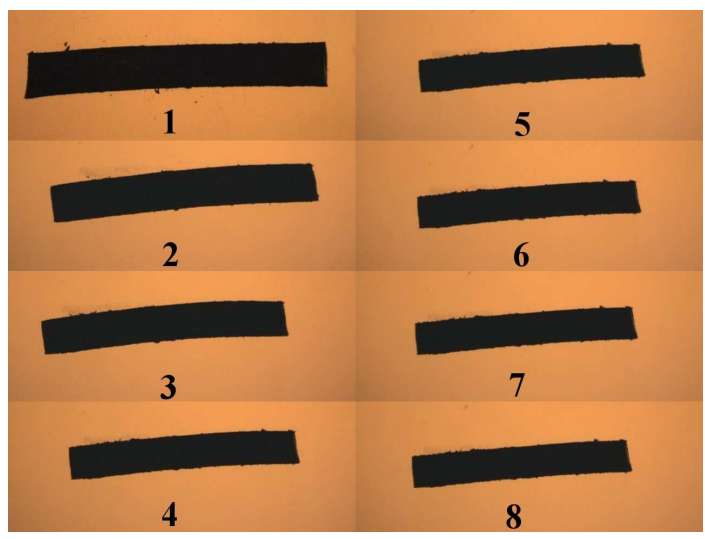

Figure 4.8. Deswelling process of poly(NIPAAm-co-MAAc) gel rod at room temperature (nominal gel rod diameter $537 \mu \mathrm{m}$ ). The gel rod was equilibrated with pH 5.6 buffer first and then transferred into pH 4.0 Buffer. The behavior was recorded as a function of time: (1) 0 min., (2) 3.0 min., (3) 5.0 min., (4) 48.0 min., (5) 66.0 min., (6) 86.0 min., (7) 111.0 min., (8) 149.0 min.

outer layer of the gel rods swell first. Accordingly, the pore sizes increase [9] and the exchange rate between the exterior and interior increases. The volume of the low $\mathrm{pH}$ buffer solution inside of the network is negligible compared to the volume of the high $\mathrm{pH}$ solution it absorbs during the swelling. This process depends on the collective diffusion of the polymer network [24] into water. When the poly(NIPAAm-co-MAA) gel rods are switched from high to low $\mathrm{pH}$, the skin layer shrinks and creates pressure to rapidly force solution out. After the initial deswelling step, further reduction of the gel volume requires the solution inside to be replaced by the outside solution and the pore size is smaller, which is very slow. The volume of the high $\mathrm{pH}$ buffer solution the gel rod contains is also larger, which contributes to the slow deswelling step. 


\subsection{Properties of the Chemomechanical Oscillator}

\subsubsection{Relationship between the pH Oscillator and the Chemome- chanical Oscillator}

Mechanical oscillations were obtained for different diameters of poly(NIPAAm-co-MAA) gel rods. The mechanical oscillations and $\mathrm{pH}$ oscillations have the same periods and are synchronized in phase, as shown in Figs. 4.9 and 4.10.

The chemomechanical oscillators exhibit a strong dependence on the size of the gel rods. For nominal diameters less than $200 \mu \mathrm{m}$, the response of the gel rods to a pH change is almost immediate. This can be clearly seen in Figs. 4.9(a)-4.9(d). When the pH switches between low and high, the size of the gel rods also switch between small and large. However, for gel rods that have nominal diameters larger than $200 \mu \mathrm{m}$, a delayed response occurred when the $\mathrm{pH}$ switched from low to high. The deswelling process is much faster than the swelling process, as shown in Figs. 4.10(a)-4.10(d). This faster deswelling phenomenon was also observed by Crook et al. [2]. This is different from the experimental observations in Sections 4.4.5 and 4.4.6, where the gel rod is switched between different $\mathrm{pH}$ buffers. During the buffer switching, mass transport determines both swelling and deswelling, which is inherently a physical process. However, for the chemomechanical oscillators, chemical processes were also involved in the deswelling process. The deswelling of the gel rod starts from the center and forces the solution out, since the $\mathrm{pH}$ drop starts from the center and propagates outwards. There is no densely collapsed layer formed as in the previously described deswelling process. Furthermore, equilibrium sizes are not reached except for the smallest gel rod of $50 \mu \mathrm{m}$ diameter.

The swelling ratio ((the maximum length - the minimum length) divided by the minimum length) of the gel rods during each oscillation cycle is dependent on the diameter of the rods, as shown in Fig. 4.11. For a $50 \mu \mathrm{m}$ diameter gel, the oscillation amplitude is almost equal to the equilibrium swelling. This is due to the large area/volume ratio, which leads to a high exchange rate of solution between the inside and outside of the gel. For the $537 \mu \mathrm{m}$ gel, the oscillation amplitude is very small, as the swelling oscillations only occur at the surface 


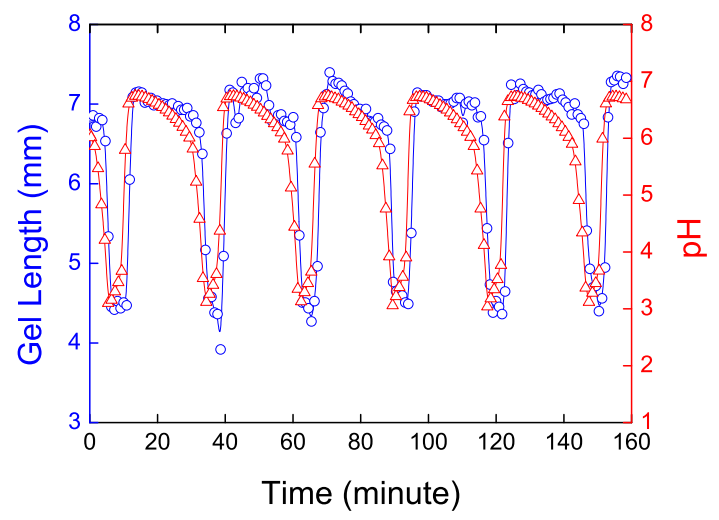

(a)

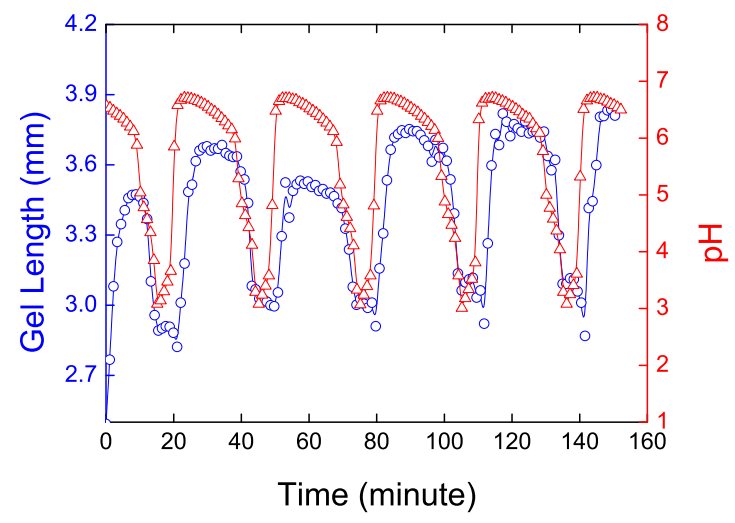

(c)

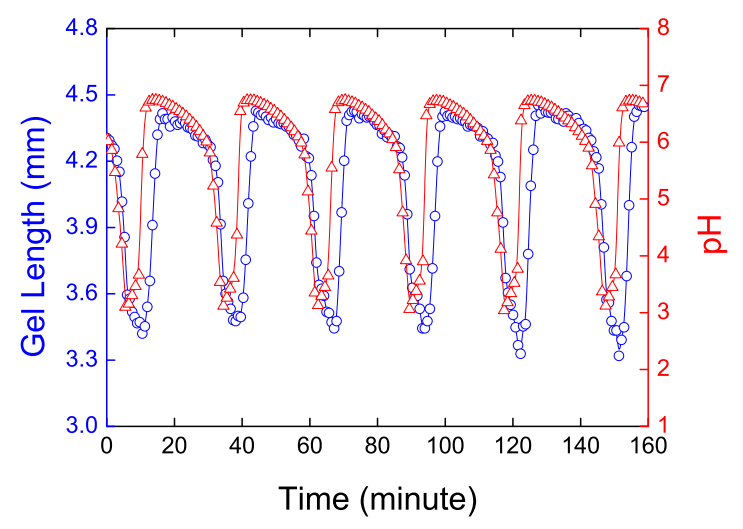

(b)

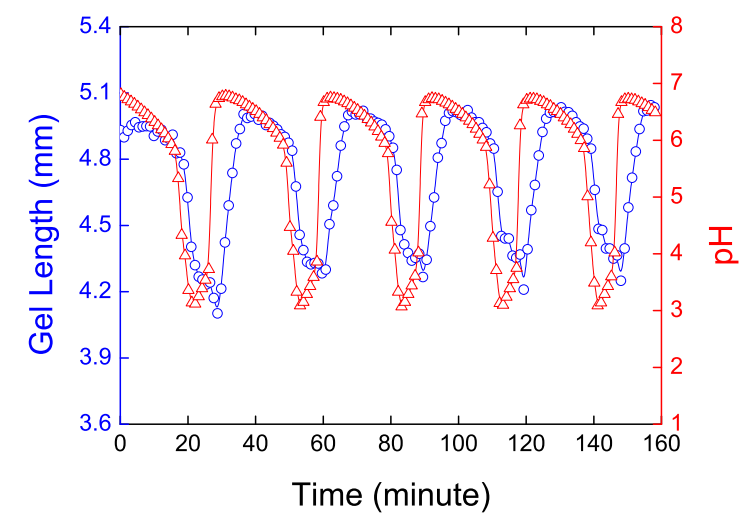

(d)

Figure 4.9. The relationships between the mechanical oscillations and the $\mathrm{pH}$ oscillations. The primary chamber volume is $31.0 \mathrm{ml}$, residence time $\tau=10.7 \mathrm{~min}$, and initial concentrations are $\left[\mathrm{Na}_{2} \mathrm{SO}_{3}\right]=0.075 \mathrm{M},\left[\mathrm{NaBrO}_{3}\right]=0.065 \mathrm{M},\left[\mathrm{K} 2 \mathrm{Fe}(\mathrm{CN})_{6}\right]=0.020 \mathrm{M},\left[\mathrm{H}_{2} \mathrm{SO}_{4}\right]$ $=0.010 \mathrm{M}$. Red triangles represent the $\mathrm{pH}$ and blue squares represent the length of the gel rod. The nominal diameters are (a) $50 \mu \mathrm{m}$, (b) $100 \mu \mathrm{m}$, (c) $150 \mu \mathrm{m}$, and (d) $180 \mu \mathrm{m}$. 


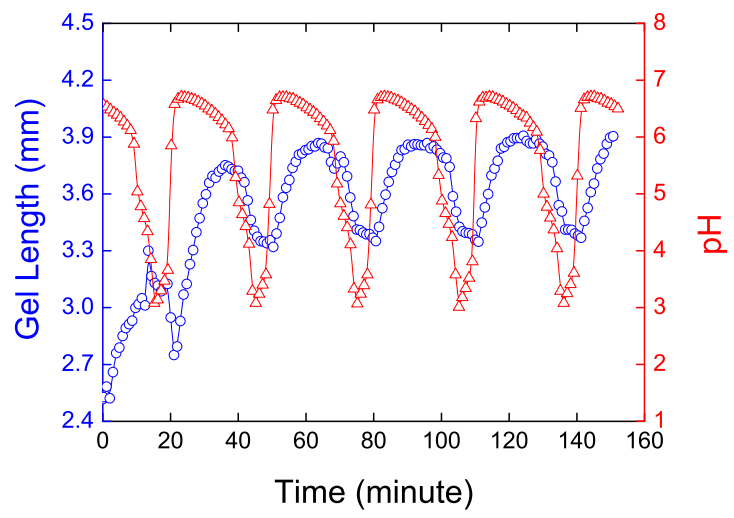

(a)

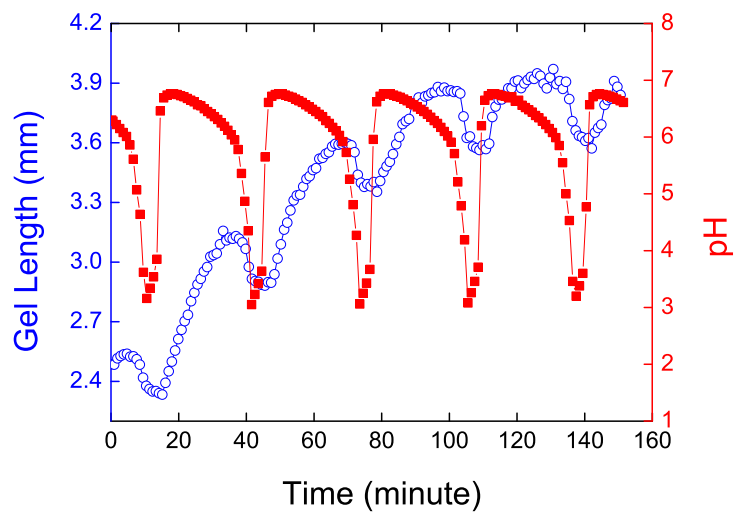

(c)

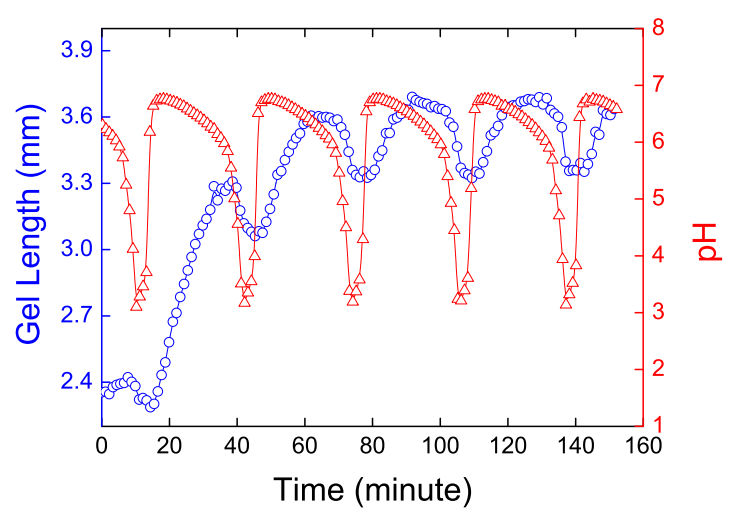

(b)

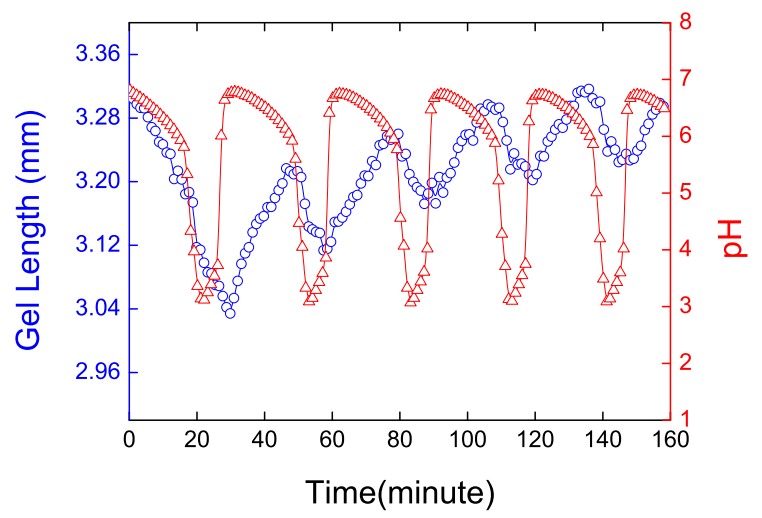

(d)

Figure 4.10. The relationships between the mechanical oscillations and the $\mathrm{pH}$ oscillations. The primary chamber volume is $31.0 \mathrm{ml}$, residence time $\tau=10.7 \mathrm{~min}$, and initial concentrations are $\left[\mathrm{Na}_{2} \mathrm{SO}_{3}\right]=0.075 \mathrm{M},\left[\mathrm{NaBrO}_{3}\right]=0.065 \mathrm{M},\left[\mathrm{K} 2 \mathrm{Fe}(\mathrm{CN})_{6}\right]=0.020 \mathrm{M},\left[\mathrm{H}_{2} \mathrm{SO}_{4}\right]$ $=0.010 \mathrm{M}$. Red triangles represent the $\mathrm{pH}$ and blue squares represent the length of the gel rod. The nominal diameters are (a) $200 \mu \mathrm{m}$, (b) $254 \mu \mathrm{m}$, (c) $319 \mu \mathrm{m}$, and (d) $537 \mu \mathrm{m}$. 
layer. For the interior of the gel, the response is too slow to follow the $\mathrm{pH}$ oscillation. The increase in length observed for the first few periods (Fig. 4.10(a) to 4.10(d)) indicates that the gel oscillation is between the swollen and an intermediate state. The center of the gel rod is not in equilibrium with the surrounding solution and the local $\mathrm{pH}$ cannot follow the solution pH. From the buffer switching experiments in Sections 4.4.5 and 4.4.6, the time for the solution exchange is much longer than the oscillation period.

\subsubsection{Buckling Behavior of the Gel}

During the chemomechanical oscillations, the gels bend during the contraction stage for all sizes of gel rods except the $537 \mu \mathrm{m}$ diameter gel rod. The buckling was initially thought to be due to solution inhomogeneity. The homogeneity of the secondary reactor chamber was analyzed in the experiments described below, along with buffer switching experiments. Both experiments indicate that buckling is a property of the gel. Microscopic inhomogeneity may arise in radical polymerizations [22, 23, 25], which can significantly affect the behavior of the gel and bending or buckling may be induced.

In addition, shrinking may induce stresses within the gel rods. Equation 4.1 describes the behavior of an ideally homogeneous material under axial pressure [26], which allows the calculation of the minimum pressure to induce buckling for a material given the column effective length factor.

$$
F=\frac{\pi^{2} E I}{K L^{2}}
$$

Here $\mathrm{F}$ is the maximum or critical force (vertical load on column), $\mathrm{E}$ is the modulus of elasticity, I is the area moment of inertia (for a circular rod, this can be calculated by Eq. 4.2), $\mathrm{L}$ is the unsupported length of column, and $\mathrm{K}$ is the column effective length factor, which depends on the conditions of end support of the column. For both ends pinned (hinged, free to rotate), $K=1.0$, and for both ends fixed, $K=0.50$. For one end fixed and the other end pinned, $\mathrm{K}=0.70$, and for one end fixed and the other end free to move laterally, $\mathrm{K}=$ 2.0 .

$$
I=\frac{1}{4} \pi r^{4}
$$




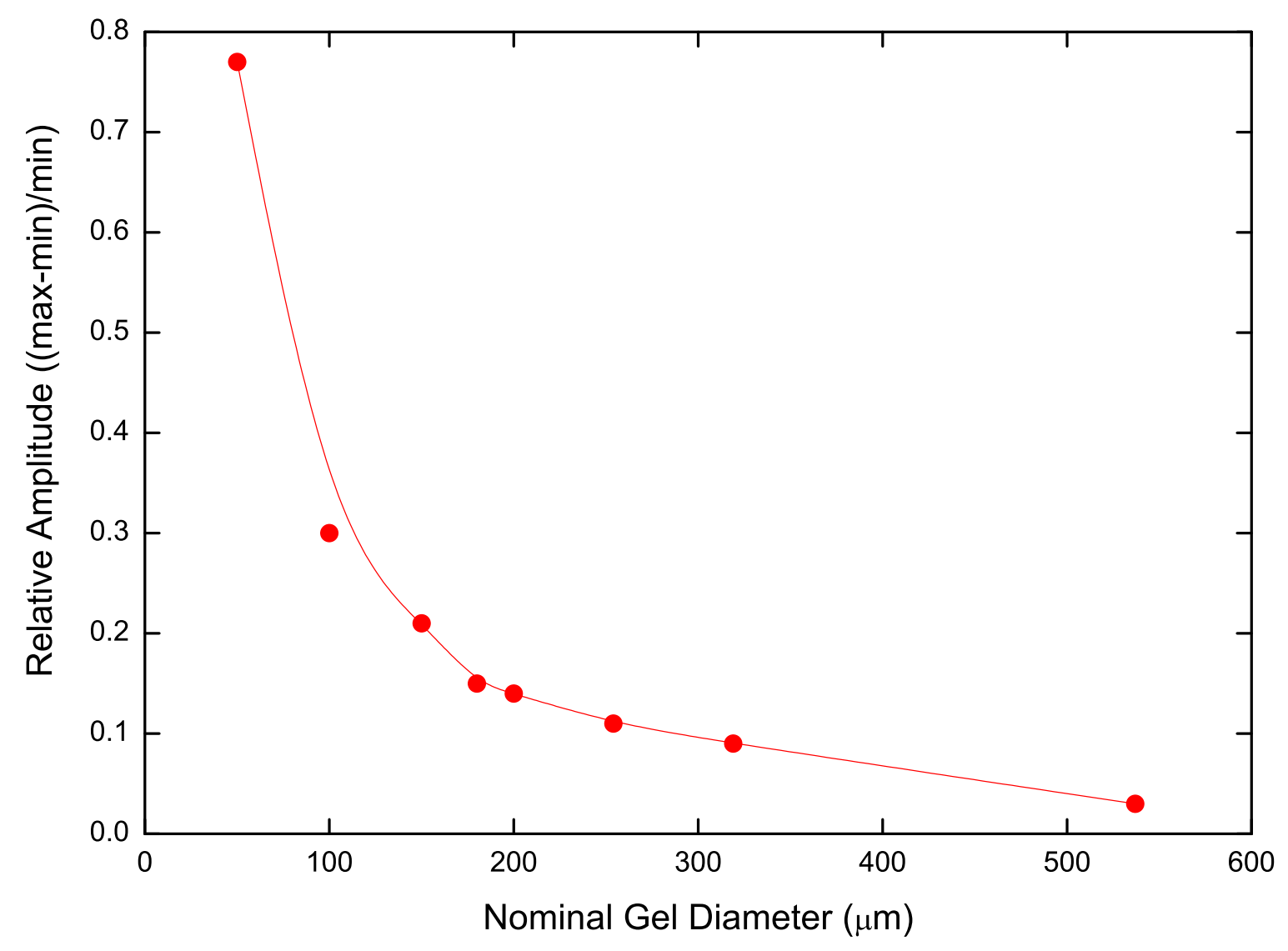

Figure 4.11. The swelling ratio dependence on the diameter of the gel rods during each oscillation period. The swelling ratio is calculated by dividing (the maximum length - the minimum length) by the minimum length of the gel rods. The nominal diameter is the diameter of the capillary used to synthesize the gel, which is about half of the gel diameter at $\mathrm{pH}$ 7.0. The primary chamber volumes is $31.0 \mathrm{ml}$, and $\tau=10.7 \mathrm{~min}$. Initial concentrations are $\left[\mathrm{Na}_{2} \mathrm{SO}_{3}\right]=0.075 \mathrm{M},\left[\mathrm{NaBrO}_{3}\right]=0.065 \mathrm{M},\left[\mathrm{K}_{2} \mathrm{Fe}(\mathrm{CN})_{6}\right]=0.020 \mathrm{M}$, and $\left[\mathrm{H}_{2} \mathrm{SO}_{4}\right]=0.010$ M. 
For the gel rods in the experiments, we can assume E, K and L are the same for all the rods since they are the same material. Therefore, the minimum force to cause the gel buckle is proportional to $r^{4}$, which means smaller diameter gel rods buckle easier than large diameter ones. Furthermore, gel rods of smaller diameter have larger deformation during the oscillation, and buckling is more likely to occur. This is in good agreement with the observed behavior of the chemomechanical oscillators.

\subsection{Conclusion}

In this chapter, we have described the development of a chemomechanical oscillator based on a $\mathrm{pH}$ oscillatory reaction and $\mathrm{pH}$-responsive poly(NIPAAm-co-MAAc) gel rods. For all the chemomechanical oscillators, mechanical oscillations were synchronized with $\mathrm{pH}$ oscillations in phase. The amplitude of the mechanical oscillations strongly depends on the diameters of the gel rods, with smaller diameter gel rods giving larger amplitude oscillations. During the mechanical oscillations, the gel rods buckle much like in the experiments switching gels between different $\mathrm{pH}$ buffer solutions. Inhomogeneity in the structure of the gel rods may give rise to preferred directions of bending. The buckling may also be due to a simple mechanical failure induced by the high stress produced during the swelling-deswelling process. 


\section{Bibliography}

[1] R. Yoshida, H. Ichijo, T. Hakuta, and T. Yamaguchi, "Self-oscillating swelling and deswelling of polymer gels," Macromol. Rapid Commun. 16, 305-310 (1995).

Online Version 54

[2] C. J. Crook, A. Smith, R. A. Jones, and A. J. Ryan, "Chemically induced oscillations in a pH-responsive hydrogel," Phys. Chem. Chem. Phys. 4, 1367-1369 (2002).

Online Version 3, 14, 54, 55, 68

[3] R. Yoshida, T. Takahashi, T. Yamaguchi, and H. Ichijo, "Self-oscillating gel," J. Am. Chem. Soc. 118, 5134-5135 (1996).

Online Version 54, 100

[4] R. Yoshida, T. Takahashi, T. Yamuguchi, H. Ichuo, and E. Kokufuta, "Self-oscillation of polymer gels coupled with the Belousov-Zhaobotinsky reaction," ACH - Models Chem. 135, 409-416 (1998).

Online Version 54

[5] R. Yoshida, T. Sakai, S. Ito, and T. Yamaguchi, "Self-oscillation of polymer chains with rhythmical soluble-insoluble changes," J. Am. Chem. Soc. 124, 8095 - 8098 (2002).

Online Version 54

[6] Y. Takeoka, M. Watanabe, and R. Yoshida, "Self-sustaining peristaltic motion on the surface of a porous gel," J. Am. Chem. Soc. 125, 13320-13321 (2003).

Online Version 54 
[7] S. Sasaki, S. Koga, R. Yoshida, , and T. Yamaguchi, "Mechanical oscillation coupled with the Belousov-Zhabotinsky reaction in gel," Langmuir 19, 5595-5600 (2003). Online Version 54

[8] V. Labrot, P. D. Kepper, J. Boissonade, I. Szalai, and F. Gauffre, "Wave patterns driven by chemomechanical instabilities in responsive gels," J. Phys. Chem. B 109, $21476-21480(2005)$.

Online Version 37, 55

[9] C. S. Brazel and N. A. Peppas, "Synthesis and characterization of thermo- and chemomechanically responsive poly(N-isopropylacrylamide-co-methacrylic acid) hydrogels," Macromolecules 28, 8016-8020 (1995).

Online Version 3, 6, 10, 14, 55, 58, 59, 60, 67

[10] M. Orban and I. R. Epstein, "Systematic design of chemical oscillators. 26. A new halogen-free chemical oscillator: The reaction between sulfide ion and hydrogen peroxide in a CSTR," J. Am. Chem. Soc. 107, 2302-2305 (1985).

Online Version 56

[11] V. Gáspár and K. Showalter, "The oscillatory Landolt reaction. Empirical rate law model and detailed mechanism," J. Am. Chem. Soc. 109, 4869-4876 (1987). Online Version 35, 37, 56

[12] E. C. Edblom, L. Györgyi, M. Orbán, and I. R. Epstein, "Systematic design of chemical oscillators. 40. A mechanism for dynamical behavior in the Landolt reaction with ferrocyanide," J. Am. Chem. Soc. 109, 4876-4880 (1987).

Online Version 35, 37, 56

[13] E. C. Edblom, Y. Luo, M. Orbán, K. Kustin, and I. R. Epstein, "Systematic design of chemical oscillators. 45. Kinetics and mechanism of the oscillatory bromate-sulfiteferrocyanide reaction," J. Phys. Chem. 93, 2722-2727 (1989).

Online Version 35, 37, 38, 40, 56 
[14] N. Okazaki, G. Rabái, and I. Hanazaki, "Discovery of novel bromate-sulfite pH oscillators with $\mathrm{Mn}^{2+}$ or $\mathrm{MnO}_{4}^{-}$as a negative-feedback species," J. Phys. Chem. A 103, 10915-10920 (1999).

Online Version 56

[15] K. M. Kovács and G. Rábai, "Large amplitude pH oscillations in the hydrogen peroxidedithionite reaction in a flow reactor," J. Phys. Chem. A 105, 9183 (2001).

Online Version 56

[16] K. Chie, N. Okazaki, Y. Tanimoto, and I. Hanazaki, "Tristability in the bromatesulfitehydrogencarbonate pH oscillator," Chem. Phys. Lett. 334, 55-60 (2001).

Online Version 56

[17] K. Kovacs, R. McIlwaine, K. Gannon, A. F. Taylor, and S. K. Scott, "Complex behavior in the formaldehyde-sulfite reaction," J. Phys. Chem. A 109, 283-288 (2005).

Online Version 56

[18] V. K. Vanag, D. G. Miguez, and I. R. Epstein, "Designing an enzymatic oscillator: Bistability and feedback controlled oscillations with glucose oxidase in a continous flow stirred tank reactor," J. Chem. Phys. 125, 194515-19427 (2006).

Online Version 56

[19] K. Kovacs, R. E. McIlwaine, S. K. Scott, and A. F. Taylor, "An organic-based pH oscillator," J. Phys. Chem. A 111, 549-551 (2007).

Online Version 56

[20] T. C. McIlvaine, "A buffer solution for colorimetric comparison," J. Biol. Chem. 49, 183-186 (1921).

Online Version 59

[21] O. Philippova, D. Hourdet, R. Audebert, and A. Khokhlov, "pH-responsive gels of hydrophobically modified poly(acrylic acid)," Macromolecules 30, 8278-8285 (1997). Online Version 3, 6, 14, 15, 60 
[22] E. S. Matsuo and T. Tanaka, "Kinetics of discontinuous volume-phase transition of gels," J. Chem. Phys. 89, 1695-1703 (1988).

Online Version 16, 62, 66, 71

[23] P. Flory, Principles of polymer chemistry (Cornell University Press, Ethaca, NY, USA, 1953). 5, 62, 71

[24] T. Tanaka and D. J. Fillmore, "Kinetics of swelling of gels," J. Chem. Phys. 70, 12141218 (1979).

Online Version 67

[25] H. Furukawa, K. Horie, R. Nozaki, and M. Okada, "Swelling-induced modulation of static and dynamic fluctuations in polyacrylamide gels observed by scanning microscopic light scattering," Phys. Rev. E 68, 013406 (2003).

Online Version 71

[26] S. P. Timoshenko and J. M. Gere, Theory of Elastic Stability (McGRAW-Hill Book Company, Columbus, OH, 1961), second edition. 71 


\section{Chapter 5}

\section{Chemomechanical Walkers}

\subsection{Introduction}

Many animals live in groups, such as in schools of fish, in flocks of birds or in swarms of insects. However, different species exhibit a diverse range of group behaviors. Theoretical studies $[1,2,3,4]$ show that such behaviors can be deduced from the behavior of the individuals and their interactions with their neighbors. The management of the group by an individual member is not necessary for the emergence of this collective behavior.

The individuals of groups exhibiting collective behavior are typically self-propelled particles (SPP) in theoretical studies. An SPP must have the ability to control its own movements and the ability to sense its neighbors and adjust its movements accordingly. The interactions with neighbors are generally designed to maintain an optimal separation distance $[1,5,6,7]$. When the distance is smaller than the optimal distance, the SPPs spread out, and when the distance is larger than the optimal distance, attraction between the SPPs will bring them closer. Based on these characteristics, different collective behaviors have been theoretically characterized and can be compared to their counterparts in the biological world [1, 2, 3].

Experimental studies of biological groups is difficult to realize. For a well-controlled experiment, a laboratory system of SPPs offers distinct advantages. We have developed a chemomechanical walker based on $\mathrm{pH}$ responsive gels and $\mathrm{pH}$ oscillatory reactions in an attempt to realize an SPP for the study of collective behavior in a laboratory environment. 


\subsection{Working Principles of the Chemomechanical Walker}

As introduced in Chapter 4, a chemomechanical oscillator can change its size and/or shape periodically. If we can develop a mechanism for a chemomechanical oscillator to translate the periodic change into unidirectional movement, then a chemomechanical walker can be successfully realized.

\subsubsection{Principles of the Ratchet and Pawl Mechanism}

The ratchet and pawl mechanism is one of the oldest methods for realizing the asymmetric operation of devices. In a ratchet device, rotation can only occur in one direction, either clockwise or counter clockwise [8]. Schematic diagrams of a toothed ratchet device and a further derived ratchet device are shown in Fig. 5.1 [8]. Figure 5.1(a) shows a basic ratchet device, which has two parts that are labeled as $\mathbf{a}$ and $\mathbf{b}$ in the figure. Part $\mathbf{a}$ is called a pawl, which is basically a protrusion against part $\mathbf{b}$. The shapes of pawls vary depending on the application. Part $\mathbf{b}$ is called a ratchet, which is a toothed gearwheel with its teeth set off at an angle. In mechanical designs, the ratchet and pawl can be held together by gravity, springs, or centrifugal forces [8]. For the ratchet device depicted in Fig. 5.1(a), parts a and b work together to allow counter clockwise rotation and to prevent clockwise rotation. Figure 5.1(b) shows a further adapted ratchet, in which the movement of the arm and catch drives the stepwise rotation of the ratchet wheel.

\subsubsection{Ratchet Mechanism of a Chemomechanical Walker}

With the ratchet model in mind, we can devise various designs to transform a chemomechanical oscillator into a walker. The most intuitive design is the gearwheel turned into a flat piece with teeth, either with angles (a) or no angles (a'), as shown in Fig. 5.2. Rather than making the gearwheel movable, we make the pawl movable. Part a does not move during the action of the ratchet, while part $\mathbf{b}$ is made of gel and changes size periodically. The two ends are conical in shape, and can either be made of gel or other material. The middle part is rod-shaped, and is made of a chemo-responsive gel. This is the engine of the chemomechanical walker. Figure 5.2 shows the working cycles of the walker. Initially, the 


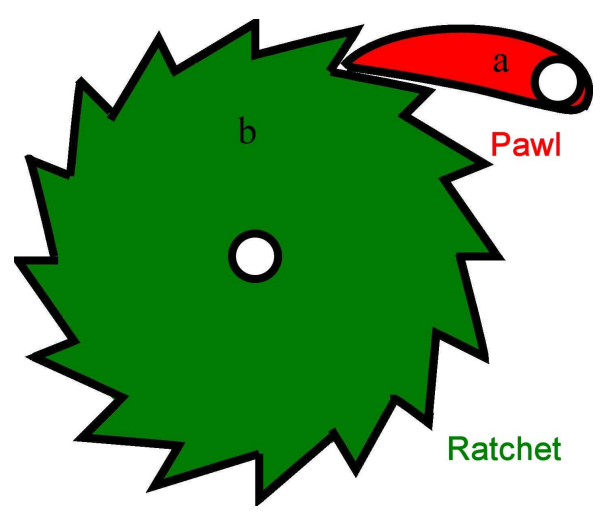

(a)

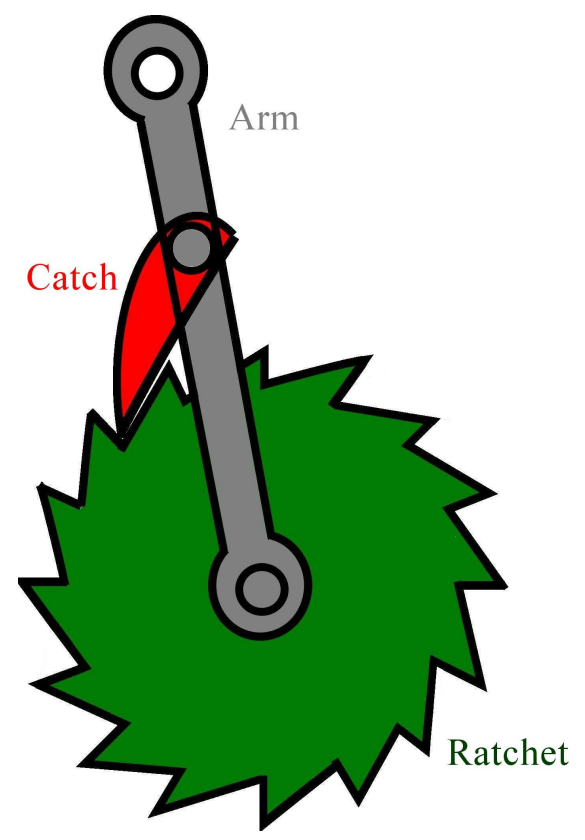

(b)

Figure 5.1. Principles of ratchet and pawl mechanism. Image is taken from [8] and was adapted and redrawn. (a) Basic design of a ratchet device. Part a is called a pawl and part $\mathbf{b}$ is called a ratchet. The pawl allows the ratchet to rotate counter clockwise only. (b) A further adapted ratchet device. The arm can go left and right, the ratchet then rotates counter clockwise step by step, driven by the movement of the catch. 
chemomechanical gel is in the contracted state, and the gel is short, as shown in Fig. 5.2(a). When the gel is expanding, the front moves forward while the end is stationary due to the restriction of the conical end with the teeth. This is illustrated in Fig. 5.2(b). After expanding, the gel will contract again. During the contraction, the teeth design of the surface does not allow the front to move backward, while the end moves forward. Comparing Figs. $5.2(\mathrm{a})$ and $5.2(\mathrm{c})$, we see that the walker moved to the right by one tooth. Similarly the next expanding cycle will move the gel front to the right by one tooth as shown in Fig. 5.2(d).

There are several requirements for the mechanism shown in Fig. 5.2 to work properly. First, the expansion/contraction of the gel must be a linear change of its size; otherwise, the ratchet model may not function. Second, the absolute length difference between the contracted state and the expanded state must be equal to or larger than the distance between two adjacent teeth. At the same time, the conical end should not be elastic.

Based on this design, the gel walker will move in a direction that is perpendicular to the teeth. This means that the direction of the walker is predefined. It is not possible for the walker to move in other directions when it meets obstacles or upon collision with other walkers. As described in Chapter 4, the mechanical gel oscillators typically buckle, and the expansion is not linear. In order to utilize the mechanism described above, further modification of the gel behavior is required.

\subsubsection{Stick-Slip Mechanism of Chemomechanical Walker}

Another design can be based on a stick-slip mechanism. Mahadevan, Daniel and Chaudhury [9] utilized PDMS films with slanted cuts to produce an asymmetric surface, allowing a piece of polyacrylamide gel to move unidirectionally. In their experiments, the polyacrylamide gel initially sticks to the PDMS surface. On application of a vibrational force, the gel moves in a direction that is perpendicular to the cuts, due to the asymmetric opening of the slanted incisions.

We may modify the above mechanism in our design of a chemomechanical walker. The mechanism is illustrated in Fig. 5.3. When the gel is expanding from the contracted state, the incisions will open and prevent movement to the left. Thus the front of the gel will move to the right. Similarly, when the gel is contracting from the expanded state, the incisions 


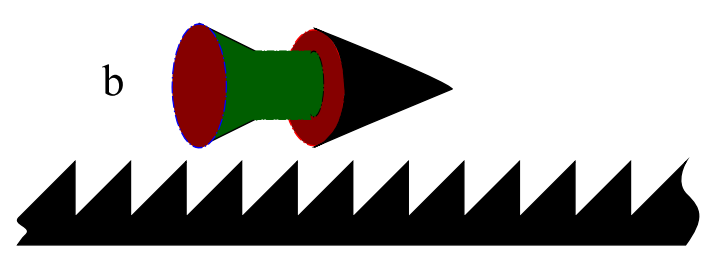

a

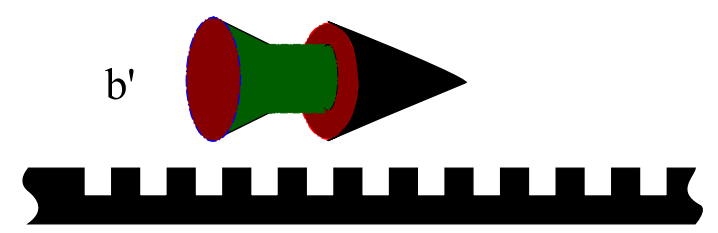

$a^{\prime}$

(a)

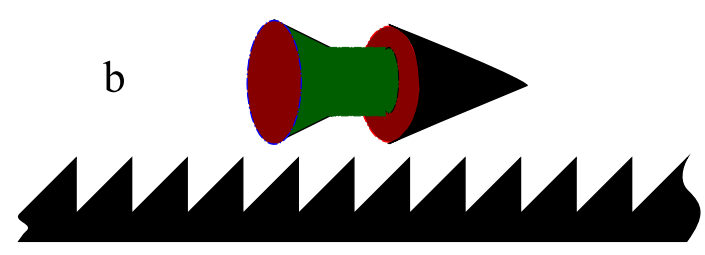

a

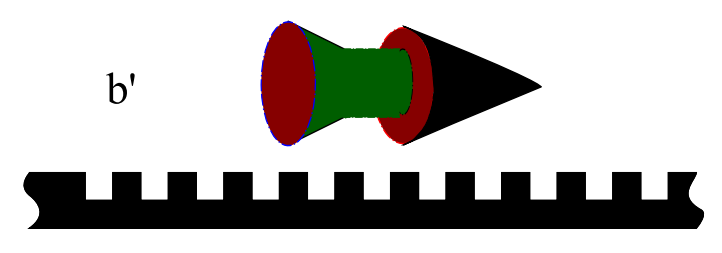

$a^{\prime}$

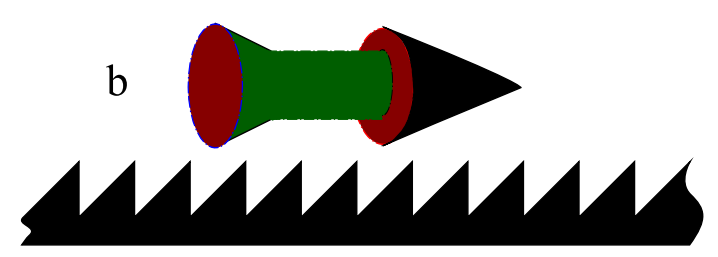

a

$b^{\prime}$
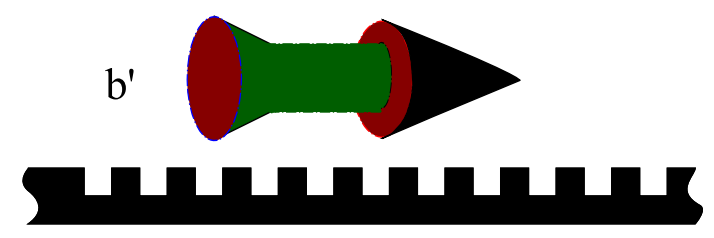

$a^{\prime}$

(b)

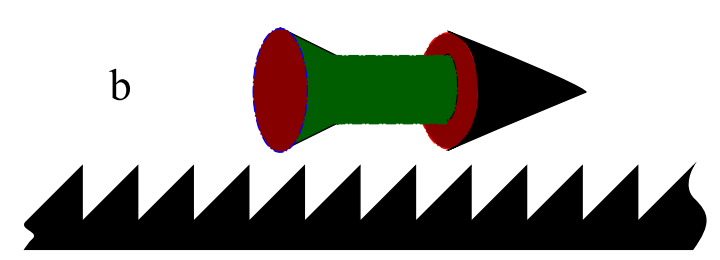

a

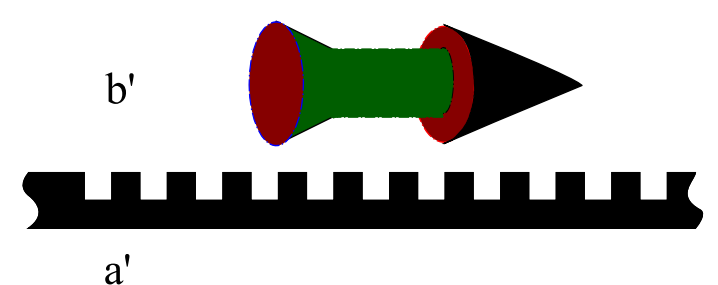

(d)

Figure 5.2. Ratchet design of a chemomechanical walker. The surface teeth can be slanted at an angle (labeled a, b, c and d) or not (labeled a', b', c' and d'). The walker continuously moves to the right when its size changes periodically. (a) Assume the gel starts from the contract state. (b) When the gel change from the contracted state to the expand state, the conical back end of the walker restricts the movement to the right only. After the length attains a maximum, the front end moves one or more teeth to the right. (c) After expanding to the maximum length, the gel starts to contract, and the back end of the walker moves to the right. Compared to the position in (a), the whole walker moves to the right by one or more teeth. (d) During the next cycle, the walker starts expanding again, and step (b) will be repeated. 


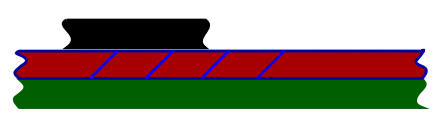

(a)

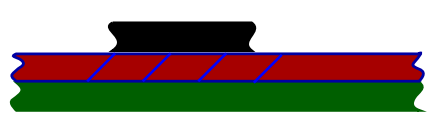

(c)

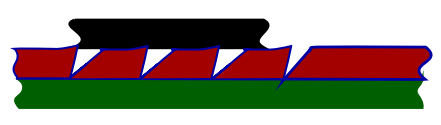

(b)

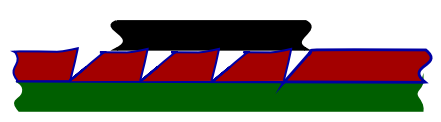

(d)

Figure 5.3. Stick-slip mechanism of a ratchet. Following the mechanical oscillation of the gel, it moves constantly to the right. (a) Initially, the gel is in the contracted state, and the slanted cuts in the PDMS surface are closed. (b) When the gel is expanding, it expands in both directions, and the force will open the cuts. The opening of the cuts prevents the gel from sliding to the left, and the gel moves to the right. (c) After the expanding stage, the gel is contracting, and the left end moves to the right. The cuts close on this step and the gel and the PDMS surface returns to the original state except that the entire gel has moved to the right. (d) The gel expands again and (b) is repeated. 
will close and the left end of the gel will move forward to the right. It is possible to have incisions on the gel with a similar mechanism as in Fig. 5.3. However, this is more difficult to realize experimentally due to the size and mechanical strength of the gel. In order to have a large swelling/deswelling ratio, the gel rod diameter must be smaller than about 250 micrometers, and the fabrication of the incisions on the gel rod is extremely difficult. Furthermore, polymer gels are generally mechanically weak, and the gel rod may break at the incisions due to the reduced mechanical strength in the expanded state.

Compared to the design in Fig. 5.2, we find that the fabrication is easier and more practical. In terms of the translational direction, the walker can not vary its course. The buckling behavior during the chemomechanical gel oscillation is also an obstacle to this design.

\subsubsection{Asymmetric Surface Friction Design of a Chemomechanical Walker}

Unlike the above designs, the third design we describe here takes advantage of the natural buckling behavior of the gel between the contracted states and expanded states. In this design, the chemomechanical gel is used as the engine and two short capillary tubes with incisions on the surface are used as legs. The chemomechanical walker can move to any direction, since the dependence on surface asymmetry is eliminated. The asymmetric frictional force between the capillary legs and the surface provide the necessary bias to produce directional movement.

The mechanism is demonstrated in Fig. 5.4. During the first half of the cycle, the gel expands and moves to the right. Ideally, the gel front moves to the right while the gel legs are stationary for this half cycle. During the second half cycle, the gel contracts towards the center of mass; the gel front moves to the left and the gel legs move to the right. Under ideal situations, the center of mass remains stationary. Compared to the beginning of the first half cycle, the gel legs, gel front and the center of mass have all moved to the right. The expanding of the gel will initiate another half cycle. During the expanding process, the center of mass and the front moves forward. During the contracting state, the center of mass 

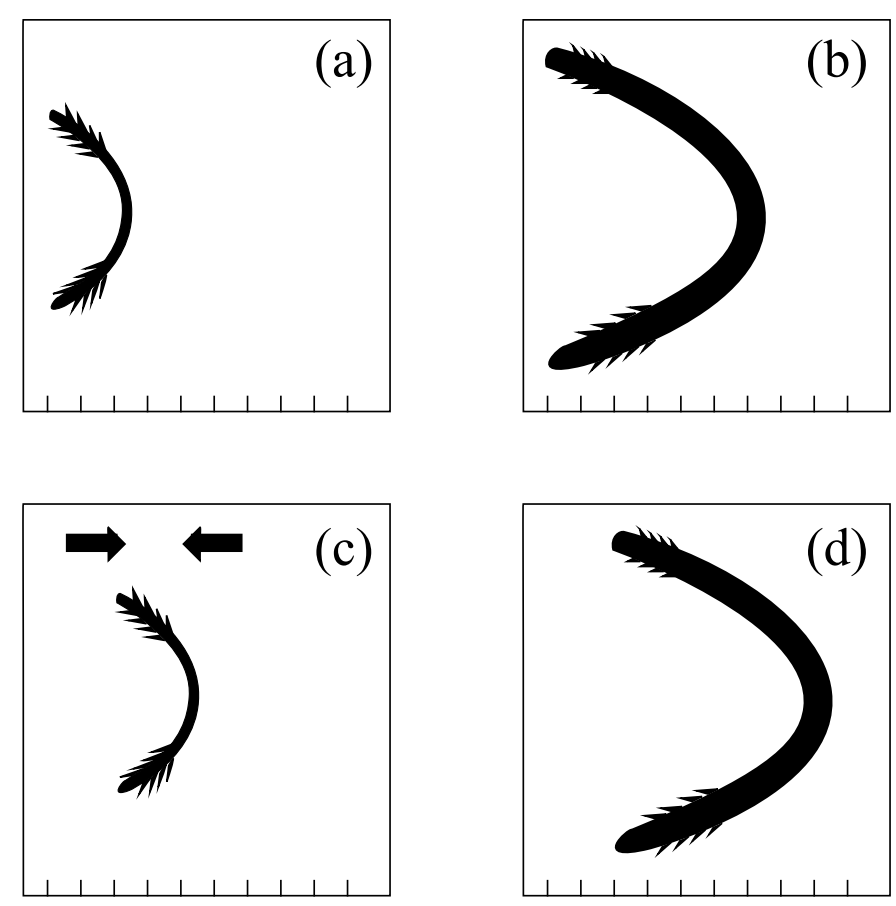

Figure 5.4. Asymmetric friction mechanism for a ratchet. During a cycle of the mechanical oscillation, the walker moves forward by a large step and backward by a small step. The net movement is equal to the difference between the two movements and the direction is to the right. (a) The walker starts in the contracted state. (b) During expansion, the front moves to the right and the back remains stationary. (c) During contraction, the front moves backward and the back moves forward. (d) The expansion is then repeated.

and the front moves backward. However, the distance the walker moves forward is greater than the distance it moves backward, and the overall movement is forward.

For this design, the gel must move on a rough surface that can provide enough frictional resistance. Multiple incisions on the legs provide better translational efficiency.

\subsection{Experimental Setup: Fabrication of the Chemomechanical Walker}

The third design described in Section 5.2 .4 became the preferred design for our experimental study. The chemomechanical walker is composed of two parts: a poly (NIPAAm-co-MAAc) 
gel rod and capillaries of the appropriate diameter. The poly (NIPAAm-co-MAAc) gel rod was synthesized by the method described in Chapter 4 . The capillaries used here are the TSP series purchased from Polymicro Inc. For the TSP capillaries, the coating is polyimide which can easily be cut with a razor blade. By carefully cutting the polyimide coating, incisions with about 200 to $300 \mu \mathrm{m}$ spacing can be created. After creating the incisions on the surface, the capillaries are cut off and are ready for use. Both ends are polished with fine sand paper to obtain a smooth cross section and to prevent cutting the polymer gel. Figure 5.5(a) shows a typical capillary leg used in the experiments. As a comparison, a grasshopper's leg is shown in Fig. 5.5(b). As we can see, the incisions on the capillary are similar to the spines on the grasshopper's leg.

Before assembly of the poly (NIPAAm-co-MAAc) gel rod with the capillary legs, we must match the gel and the capillary. The matching technique requires the inner diameter of the capillary to be between $4 / 5$ and $5 / 6$ of the inner diameter of the capillary used to make the gel rod. With this technique, the gel and the capillary legs will not become separated during the mechanical oscillation of the gel. The gel rod is immersed into $0.72 \mathrm{M} \mathrm{H}_{2} \mathrm{SO}_{4}$ until equilibrium is established. With the aid of a stereo microscope, the gel rod is inserted into the capillary legs. The assembled chemomechanical walker is then placed into water or pH buffers so the gel rod will swell. The chemomechanical walker is then ready for use.

\subsection{Experimental Setup: Reactor}

In order for the gel walker to function, an unstirred reactor must be used. As described in Chapters 3 and 4, a flow through reactor can meet this requirement. The gel walker is placed into the secondary chamber to minimize perturbations from the flow. However, the secondary chamber is made of glass and the surface is smooth. Hence, a rough surface made of sand paper molded PDMS film was prepared. Under these experimental conditions, the friction is sufficient to maintain the walker stationary in the fluid flow. The friction also permits the walker to move in a particular direction as the chemomechanical oscillations occur. 


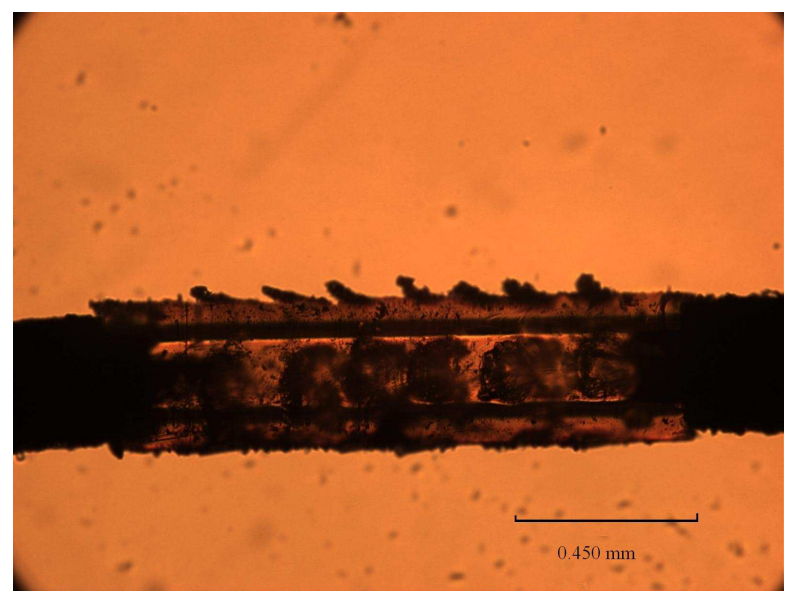

(a)

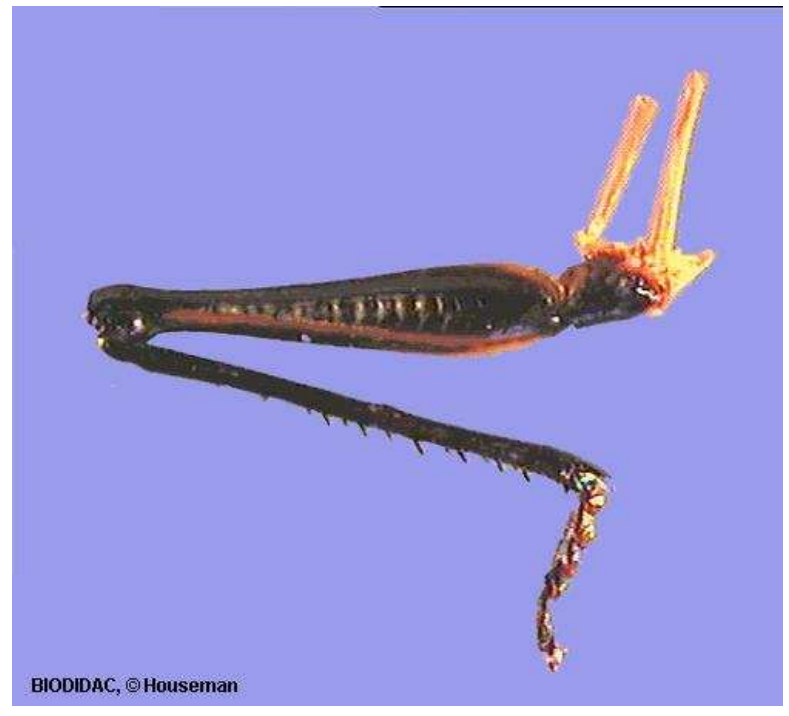

(b)

Figure 5.5. (a) Image of a chemomechanical walker leg, taken with a CCD camera attached to a 10X microscope. The incisions were cut with a razor blade. The scale bar in the image is 450 micrometers. The incisions induce an asymmetric frictional force when moving in different directions. (b) Leg of a grasshopper. Image was taken from http://www.esu.edu/ milewski/intro_biol_two/lab_12_annel_arthro. 


\subsection{Results}

\subsubsection{Movement of the Chemomechanical Walker}

Experiments demonstrated that the asymmetric frictional force generated robust and predictable movement. Figure 5.6 shows the behavior of the chemomechanical walker within one period. The gel begins in the flexed position at time $\mathrm{t}=0 \mathrm{~s}$ and undergoes a flexing sequence before arriving back at its maximally flexed shape at $t=2200 \mathrm{~s}$. All the changes are reversible and are repeated every cycle.

Figure 5.7 shows the spatiotemporal behavior of the chemomechanical walker. Images of the walker are superimposed at equal time intervals. The time between subsequent images of the walker is one period.

\subsubsection{Speed of the Chemomechanical Walker}

The relationships between the moving speed of the chemomechanical walker and solution $\mathrm{pH}$ is displayed in Fig. 5.8, which shows that for each oscillation period, the walker has two peak speeds. The peak speeds coincide with the sharp pH changes. The first peak appears with the $\mathrm{pH}$ change from low to high and the second peak appears with the $\mathrm{pH}$ change from high to low. The first peak is always higher than the second peak and the moving directions are opposite to each other. The pH oscillation and the mechanical oscillation are in phase, as discussed in Chapter 4. For the first half of the cycle, the chemomechanical walker increases in size as the $\mathrm{pH}$ rises and it moves forward. For the second half of the cycle, the gel size decreases following the decrease of the $\mathrm{pH}$. The gel front then moves backward by a small amount and the gel back moves forward. The movement in this half cycle is negative. However, the overall movement for the cycle is positive. Figure 5.9 shows a trace of the center of the chemomechanical walker front. The forward-backward-forward moving pattern matches the analysis above.

A qualitative analysis of the moving cycle can help us understand the behavior observed in Fig. 5.8. Figure 5.10 shows the positions of the chemomechanical walker during a working cycle. Initially, the gel starts from its contracted state and we denote its front position as 1. 


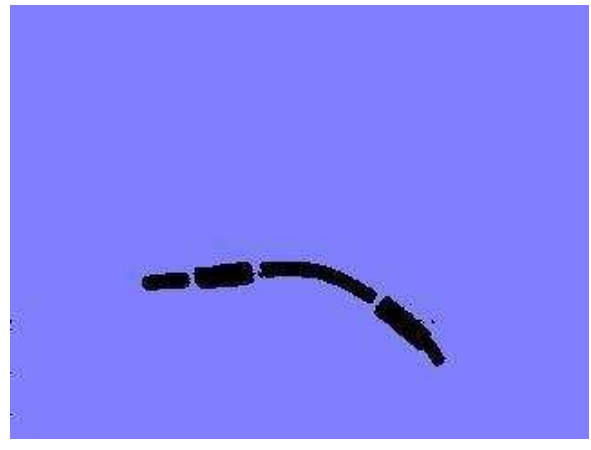

(a)

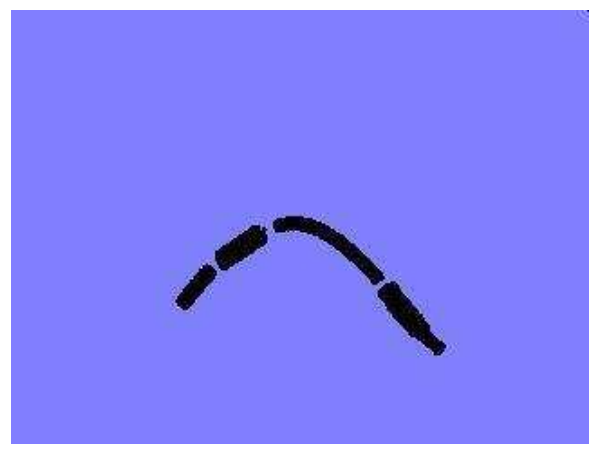

(c)

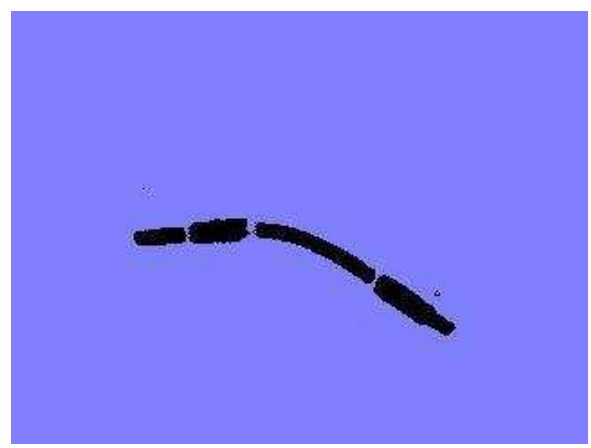

(e)

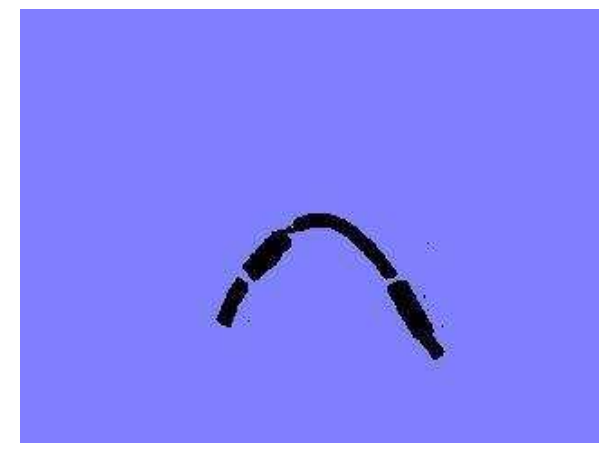

(b)

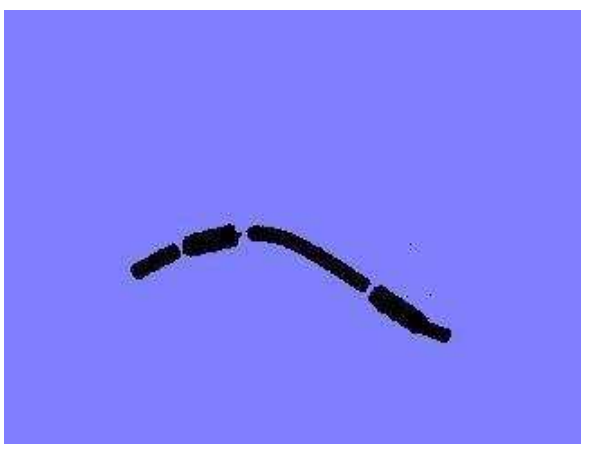

(d)

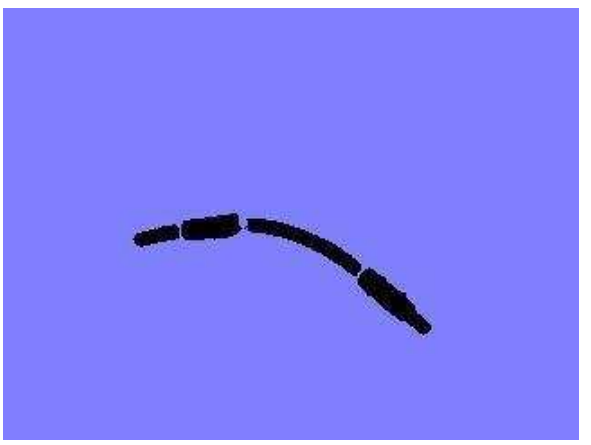

(f)

Figure 5.6. Shape of gel during one oscillatory period. Time $\mathrm{t}=0 \mathrm{~s}(1), 156 \mathrm{~s}(2), 234 \mathrm{~s}(3)$, $351 \mathrm{~s}(4), 701 \mathrm{~s}(5), 1168 \mathrm{~s}(6)$. Initial concentrations: $\left[\mathrm{Na}_{2} \mathrm{SO}_{3}\right]=0.075 \mathrm{M},\left[\mathrm{NaBrO}_{3}\right]=$ $0.065 \mathrm{M},\left[\mathrm{K}_{2} \mathrm{Fe}(\mathrm{CN})_{6}\right]=0.020 \mathrm{M},\left[\mathrm{H}_{2} \mathrm{SO}_{4}\right]=0.010 \mathrm{M}$. Residence time $=12.7 \mathrm{~min}$. 


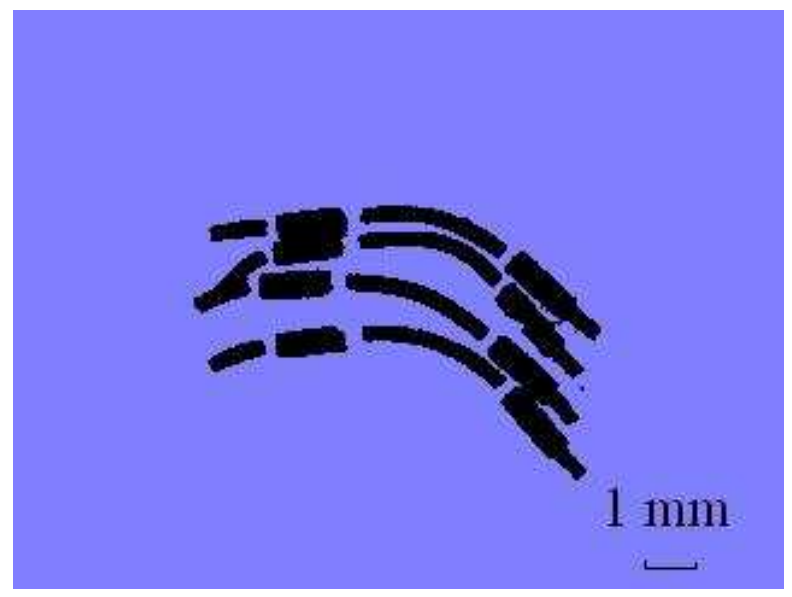

Figure 5.7. Space-time plot of gel movement comprised of superimposed images. The chemical oscillation period is $20 \mathrm{~min}$. The time between each image is $20 \mathrm{~min}$. Initial concentrations: $\left[\mathrm{Na}_{2} \mathrm{SO}_{3}\right]=0.075 \mathrm{M},\left[\mathrm{NaBrO}_{3}\right]=0.065 \mathrm{M},\left[\mathrm{K}_{2} \mathrm{Fe}(\mathrm{CN})_{6}\right]=0.020 \mathrm{M},\left[\mathrm{H}_{2} \mathrm{SO}_{4}\right]=0.010$ M. Residence time $=12.7 \mathrm{~min}$.

During the expansion, the gel grows and due to the incisions on the two legs, the gel front moves forward by $2 x$ in the horizontal direction, as shown in Fig. 5.10. This distance is only a portion of the total length increment, and it can be roughly estimated with Eq. 5.1. The position corresponds to the first peak in the speed plotted in Fig. 5.8. We denote the current position as 2. During the shrinking stage, the gel front moves backward by $x$. This corresponds to the second peak in the speed plotted in Fig. 5.8. The second peak is about half of the first peak and the movement is in the opposite direction. The net movement is $\mathrm{x}$.

$$
2 x=\frac{1}{2} d \cos \left(\frac{1}{2} \alpha\right)
$$

Equation 5.1 is used to estimate the movement from the length change of the gel. In Eq. $5.1, d$ is the the total length increment from the contracted state to the expanded state and $\alpha$ is the buckling angle.

\subsubsection{Selection of the Chemomechanical Walker Length}

Equation 5.1 indicates that longer gels should move faster. However, longer gels buckle in more than one place, and the movement loses its directionality. Due to size limitations of 


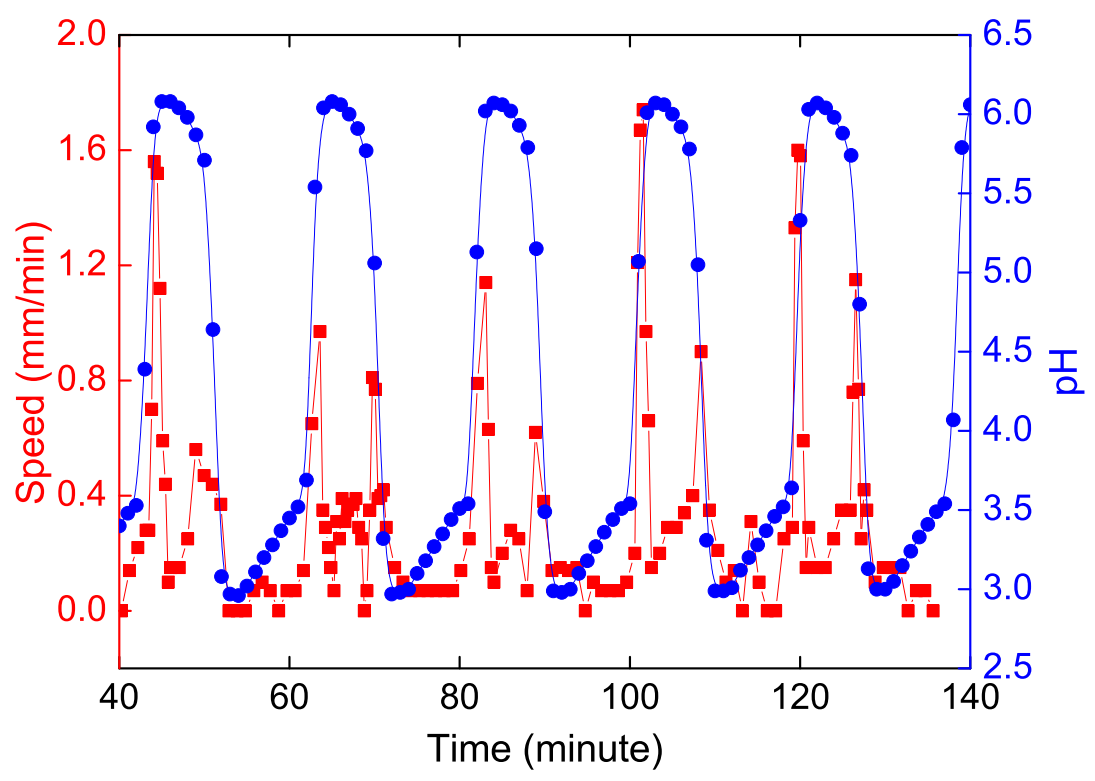

(a)

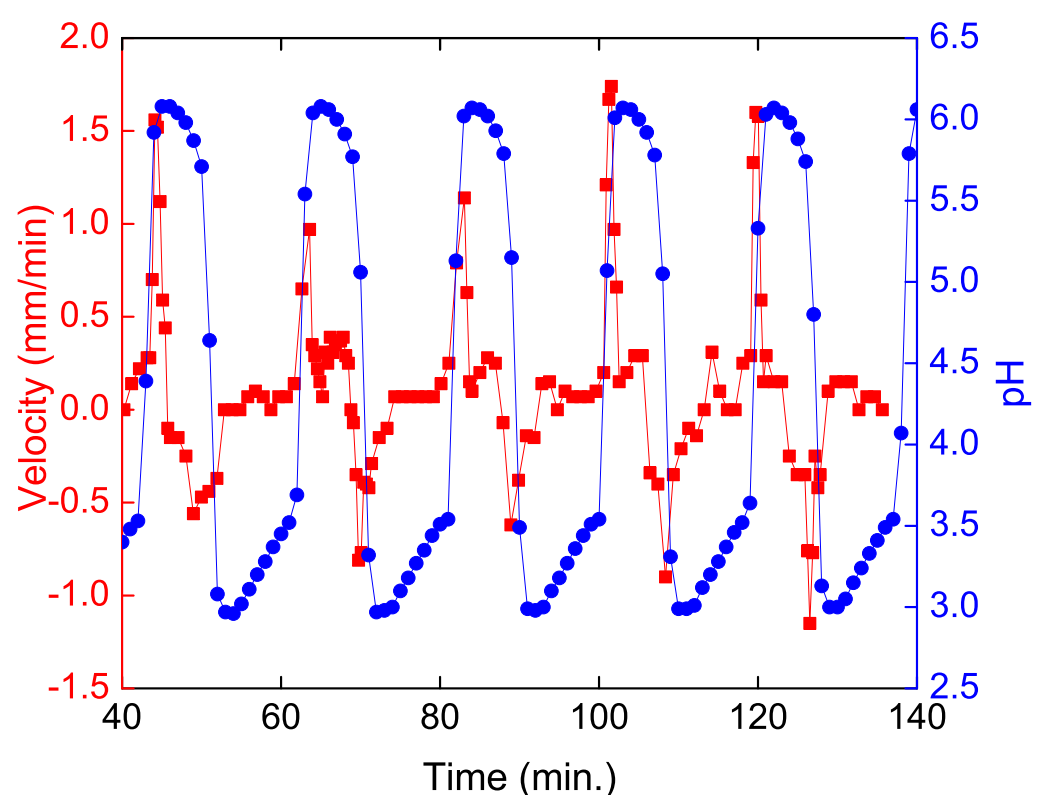

(b)

Figure 5.8. The relationship between the translational speed (a) and velocity (b) of the chemomechanical walker (squares) and solution $\mathrm{pH}$ (circles). Initial concentrations: $\left[\mathrm{Na}_{2} \mathrm{SO}_{3}\right]$ $=0.075 \mathrm{M},\left[\mathrm{NaBrO}_{3}\right]=0.065 \mathrm{M},\left[\mathrm{K}_{2} \mathrm{Fe}(\mathrm{CN})_{6}\right]=0.020 \mathrm{M},\left[\mathrm{H}_{2} \mathrm{SO}_{4}\right]=0.010 \mathrm{M}$. Residence time $=12.7 \mathrm{~min}$. Gel nominal diameter is $180 \mu \mathrm{m}$. 


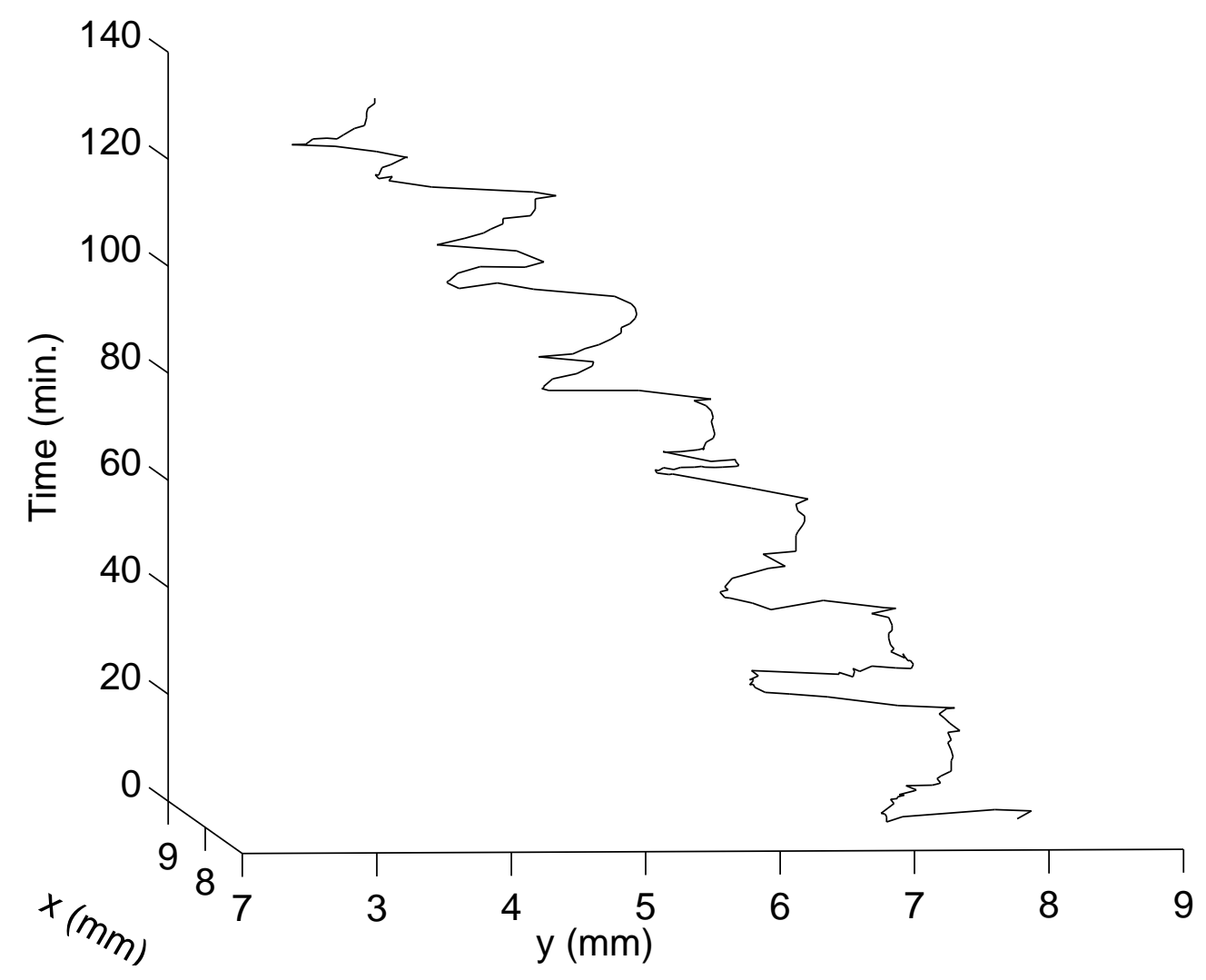

Figure 5.9. The path of the chemomechanical walker front. The walker lies in the $x-y$ plane, the $\mathrm{z}$ axis represents time. The walker front moves forward and then backward; however, the distance the walker moves forward is larger than that it moves backward. Initial concentrations: $\left[\mathrm{Na}_{2} \mathrm{SO}_{3}\right]=0.075 \mathrm{M},\left[\mathrm{NaBrO}_{3}\right]=0.065 \mathrm{M},\left[\mathrm{K}_{2} \mathrm{Fe}(\mathrm{CN})_{6}\right]=0.020 \mathrm{M}$, $\left[\mathrm{H}_{2} \mathrm{SO}_{4}\right]=0.010 \mathrm{M}$. Residence time $=12.7 \mathrm{~min}$. Gel nominal diameter is $180 \mu \mathrm{m}$. 


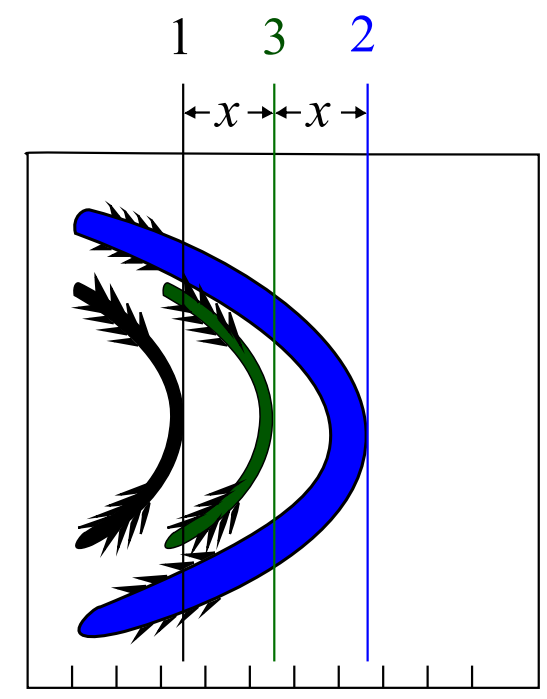

Figure 5.10. Analysis of the movement of the chemomechanical walker. The walker initially is in the contracted state in position 1 (black). The position of the center of the front is monitored. During the expanding process, the gel volume increases and the chemomechanical walker front moves into position 2 (blue). During the contraction, the gel shrinks and the chemomechanical walker front moves back to position 3 (green). 
the secondary chamber and limitations in the walker fabrication, no systematic studies have been carried out to determine the optimal length of the gel walker.

\subsection{Comparison with Other Chemomechanical Walkers}

Yoshida and co-workers developed two chemomechanical walkers in 2007 and 2008 [10, 11, 12]. The first walker was made of a ruthenium containing gel, which is a type II chemomechanical oscillator described in Chapter 4. The walker moved on a slanted surface similar to the one shown in Fig. 5.2. Different bending angles in the vertical direction occurred during the chemomechanical oscillations in catalyst-free BZ solution, and the asymmetric surface directed the walker into the predefined direction [10]. The second walker, which was developed in 2008, was made from the same gel. The gel served as the driving force instead of a walker. During the chemomechanical oscillations, waves were produced in the gel and small particles were driven by the force in a specified direction.

There are two major differences between the above walkers and the walker developed in this lab. First, the walker described in this work can move in a two-dimensional space, which means that upon collision the walker can change direction, since the direction of movement does not depend on the surface. The walkers developed by Yoshida and co-workers can

only move in one-dimensional space and the direction is predefined by the surface [10] or chemomechanical waves in the gel $[11,12]$. Second, our walker is made from a type I chemomechanical oscillator, with oscillations occurring in the surrounding solution, while the walkers in Yoshida's work were made from a type II chemomechanical oscillator, and oscillations occur only inside the gel and the solution environment is quiescent.

\subsection{Conclusion}

By utilizing the asymmetric frictional design, a chemomechanical walker was successfully developed in 2005. The walker moved in a forward-backward-forward pattern. The moving pattern was confirmed by analyzing the path of the gel and the relationship of the movement 
with the solution $\mathrm{pH}$. The movement is highly directional; however, the moving direction can be changed upon collision with another walker. This design can be used for the other type of chemomechanical oscillators, such as type II or III in Chapter 4, and new class of chemical walkers can be constructed. 


\section{Bibliography}

[1] I. D. Couzin, J. Krause, R. James, G. D. Ruxton, and N. R. Franks, "Collective memory and spatial sorting in animal groups," J. Theor. Biol. 218, 1 (2002).

Online Version 78

[2] N. Shimoyama, K. Sugawara, T. Mizuguchi, Y. Hayakawa, and M. Sano, "Collective motion in a system of motile elements," Phys. Rev. Lett. 76, 3870-3873 (1996).

Online Version 78

[3] A. Czirok, H. E. Stanley, and T. Vicsek, "Spontaneously ordered motion of self-propelled particles," J. Phys. A, Math. Gen. 30, 1375-1385 (1997).

Online Version 78

[4] J. Toner and Y. Tu, "Flocks, herds and schools: A quantitative theory of flocking," Phys. Rev. E 58, 4828 (1998).

Online Version 78

[5] J. Krause and G. D. Ruxton, Living in groups (Oxford University Express, Oxford, UK, 2002). 78

[6] B. Patridge and T. J. Pitcher, "The sensory basis of fish schools: Relative role of lateral line and vision," J. Comp. Physiol. A 135, 315-325 (1980).

Online Version 78

[7] B. Patridge, "The structure and function of fish schools," Sci. Am. 245, 90 (1982). 78 
[8] F. H. Rockett, "Ratchet," McGraw-Hill's Access Science Encyclopedia of Science and Technology Online.

Online Version 79, 80

[9] L. Mahadevan, S. Daniel, and M. K. Chaudhury, "Biomimetic ratcheting motion of a soft, slender, sessile gel," Proc. Natl. Acad. Sci. USA 101, 23-26 (2004).

Online Version 81

[10] S. Maeda, Y. Hara, T. Sakai, R. Yoshida, and S. Hashimoto, "Self-walking gel," Adv. Mater. 19, 3480-3484 (2007).

Online Version 94

[11] S. Maeda, Y. Hara, R. Yoshida, and S. Hashimoto, "Peristaltic motion of polymer gels," Angew. Chem. Int. Ed. 47, 6690-6693 (2008).

Online Version 94

[12] E. Wilson, "Polymer shrinks and swells," Chem. Eng. News 86, 9 (2008).

Online Version 94 


\section{Chapter 6}

\section{Spontaneous Wave Activity in Groups of Catalytic Particles}

Quorum sensing refers to density dependent behavior found in many different bacteria species. In a more general context, quorum sensing can be viewed as a change in qualitative behavior induced by coupling between individuals, arising from chemical signaling. In this work, we develop a quorum sensing system based on excitable and oscillatory catalytic particles. The system is composed of a population of ferroin-loaded ion-exchange beads in a catalyst-free Belousov-Zhabotinsky solution, where the signaling can be local or global. Local coupling is achieved by the diffusion of chemical species, while global coupling occurs with the exchange of signaling species between the particles and the well-mixed solution.

In this chapter, we present a study of the locally coupled system. Wave behavior develops in quasi-circular groups of individually excitable beads when the group exceeds a critical size. Numerical simulations with a particle model [1] based on the ZBKE mechanism for the BZ reaction were carried out. The results for globally coupled system will be presented in Chapter 7. The basic chemistry of the Belousov-Zhabotinsky reaction will first be introduced.

\subsection{The Belousov-Zhabotinsky (BZ) Reaction}

The Belousov-Zhabotinsky (BZ) reaction was discovered by Belousov in 1958 and was further developed by Zhabotinsky $[2,3,4]$. The reaction is generally considered to be the catalytic 


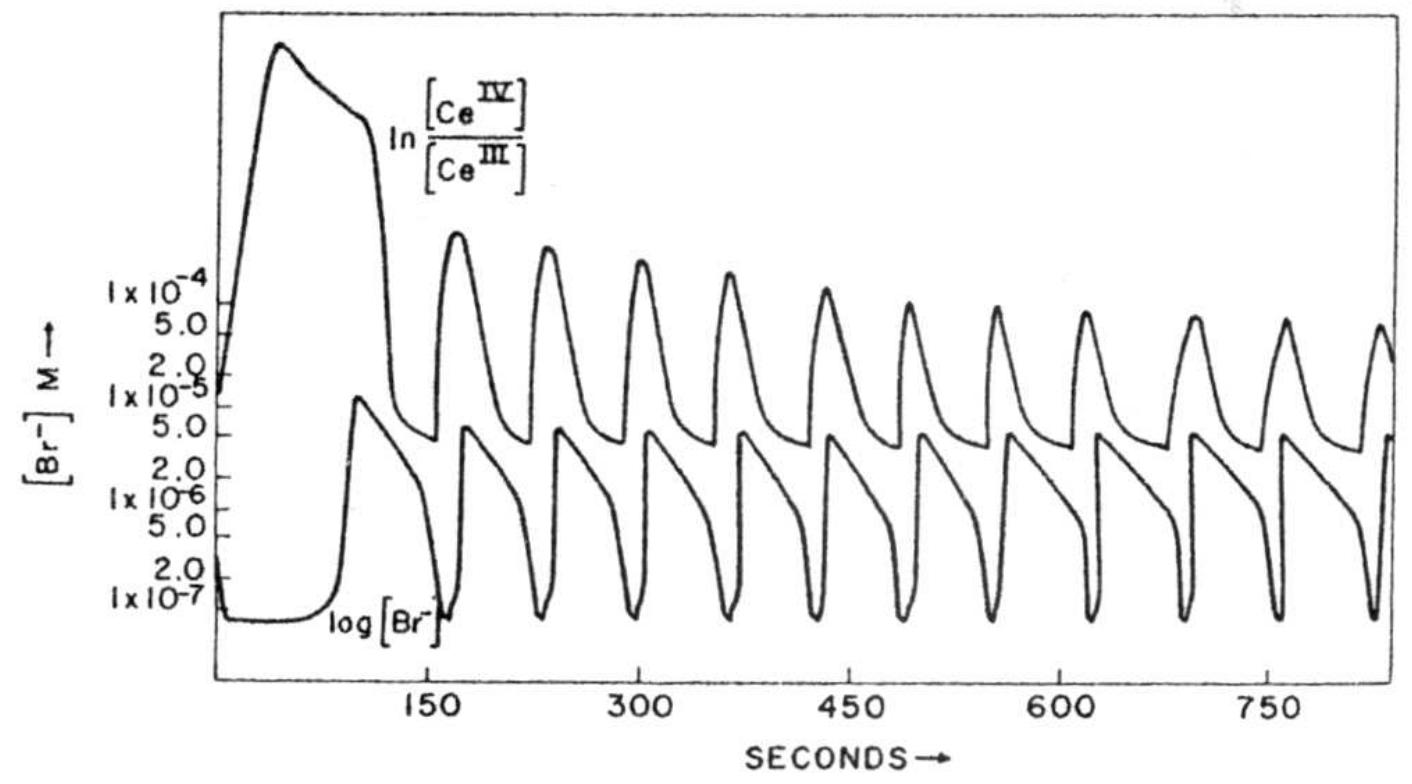

Figure 6.1. Chemical oscillations during the BZ reaction (figure from Ref. [5]). Initial concentrations were $\left[\mathrm{CH}_{2}(\mathrm{COOH})_{2}\right]_{0}=0.13 \mathrm{M},\left[\mathrm{KBrO}_{3}\right]_{0}=0.063 \mathrm{M},\left[\mathrm{Ce}\left(\mathrm{NH}_{4}\right)_{2}\left(\mathrm{NO}_{3}\right)_{5}\right]_{0}=$ $0.005 \mathrm{M},\left[\mathrm{H}_{2} \mathrm{SO}_{4}\right]_{0}=0.8 \mathrm{M}$.

oxidation of an organic acid (such as malonic acid or citric acid) by bromate ions under acidic conditions. It involves a large number of intermediates in a complex mechanism. The basic features of the reaction are well explained by the FKN mechanism developed by Field, Körös and Noyes [5] in 1972.

In the FKN mechanism, the overall reaction is divided into three processes:

Process A (consumption of $\mathrm{Br}^{-}$):

$$
\mathrm{BrO}_{3}^{-}+2 \mathrm{Br}^{-}+3 \mathrm{H}^{+} \rightarrow 3 \mathrm{HOBr}
$$

Process B (autocatalytic reaction of bromous acid with the oxidation of the catalyst):

$$
\mathrm{BrO}_{3}^{-}+\mathrm{HBrO}_{2}+2 \mathrm{M}_{\mathrm{red}}+3 \mathrm{H}^{+} \rightarrow 2 \mathrm{HBrO}_{2}+2 \mathrm{M}_{\mathrm{ox}}+\mathrm{H}_{2} \mathrm{O}
$$

Process C (organic acid oxidation with reduction of the catalyst):

$$
2 \mathrm{M}_{\mathrm{ox}}+\mathrm{BrMA} \rightarrow \mathrm{fBr}^{-}+2 \mathrm{M}_{\mathrm{red}}+2 \mathrm{CO}_{2}+5 \mathrm{H}^{+}
$$


The BZ reaction can be carried out in different configurations, such as homogeneous solutions $[5,6,7]$, gel systems $[8,9]$, or catalyst-loaded ion-exchange beads $[10,11,12]$. Depending on the recipe, the system can lie in an oscillatory state or a steady state. When the system is in the oscillatory state, the concentrations of the intermediate species $\mathrm{HBrO}_{2}, \mathrm{Br}^{-}$, and the catalyst periodically oscillate during the reaction, as shown in Fig. 6.1. When the system lies in a steady state, the concentrations of the intermediate species and catalyst are constant. Numerical studies of the BZ system are typically carried out with the Oregonator model, which was introduced by Field and Noyes in 1974 [13], or with one of the many modified versions.

\subsection{Properties of Individual Catalyst-Loaded Particles}

\subsubsection{Catalyst Particle Preparation}

The catalyst-loaded particles were prepared according to the recipe in Ref. [1]. Cation exchange beads, Bio-Rad analytical grade 50W-X (50-100 mesh), were purchased from Fisher Scientific and were loaded with the ferroin catalyst for the BZ reaction by stirring the beads in ferroin solutions overnight. The beads were filtered and washed with distilled water followed by $0.1 \mathrm{M} \mathrm{H}_{2} \mathrm{SO}_{4}$ solution. The loaded beads were allowed to dry for 24 hours and were then stored in bottles. The catalyst concentration was calculated to be $1.7 \times 10^{-5} \mathrm{~mol} \mathrm{~g}^{-1}$.

\subsubsection{Properties of Uncoupled Particles}

When the concentrations of malonic acid, bromide, and sulfuric acid are maintained constant, the catalyst particles remain in a steady state or oscillatory state depending on the concentration of bromate, as indicated in Fig. 6.2. Figure 6.2(a) shows the fraction of oscillatory particles at different bromate concentrations. When the concentration of bromate is below $0.33 \mathrm{M}$, none of the particles oscillated, with all remaining in a steady state. Greyscale analysis indicated that there was no color change during a 20 min period, as shown in Fig. 6.2(d). When the concentration of bromate was above $0.33 \mathrm{M}$, some of the particles began to oscillate. However, when the bromate concentration was between $0.33 \mathrm{M}$ and $0.39 \mathrm{M}$, only 


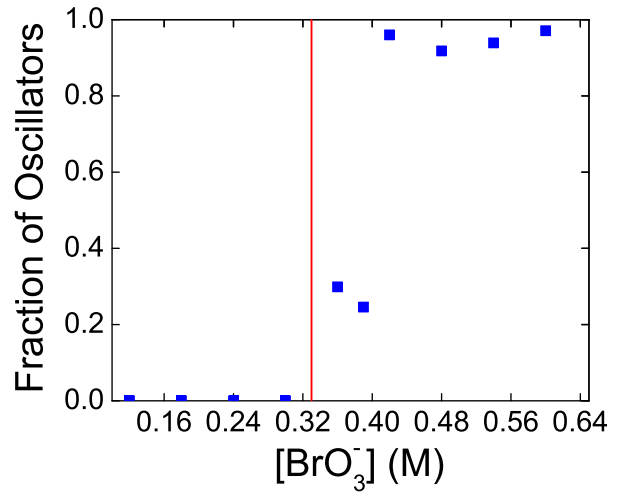

(a)

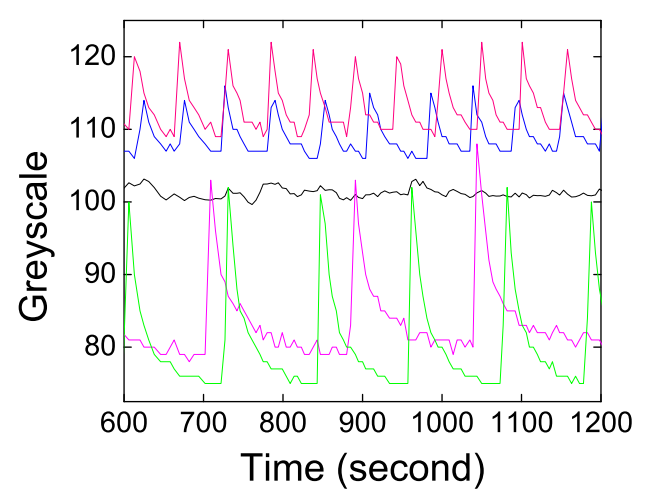

(c)

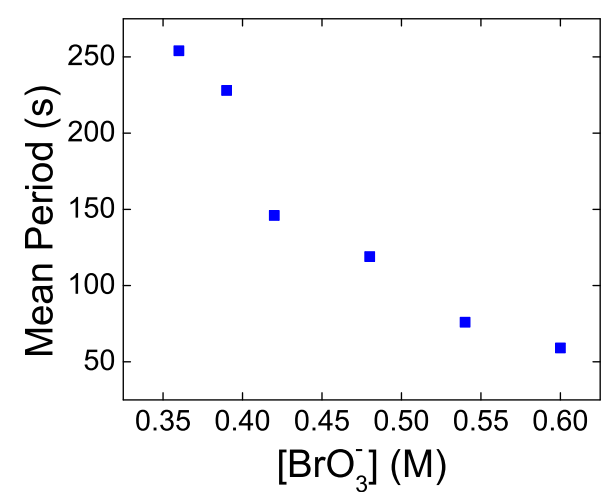

(b)

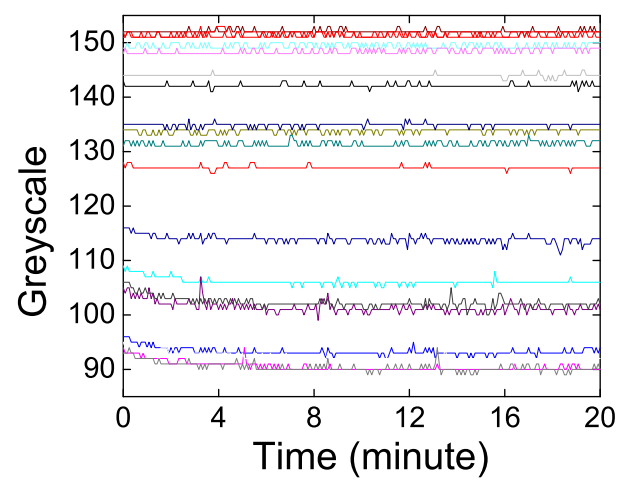

(d)

Figure 6.2. Experimental behavior of individual beads. The beads are positioned at least $5 \mathrm{~mm}$ from any nearest neighbor beads. The reaction mixture concentrations are $[\mathrm{NaBr}]=$ $0.10 \mathrm{M},[\mathrm{MA}]=0.20 \mathrm{M},\left[\mathrm{H}_{2} \mathrm{SO}_{4}\right]=0.60 \mathrm{M}$, and $\left[\mathrm{NaBrO}_{3}\right]$ with concentrations shown in panel (a) and (b). For panel (c) and (d), $\left[\mathrm{NaBrO}_{3}\right]$ is $0.51 \mathrm{M}$ and $0.30 \mathrm{M}$. The concentration of ferroin is $1.7 \times 10^{-5} \mathrm{~mol} \mathrm{~g}^{-1}$. (a) The fraction of oscillatory beads. The vertical red line indicates the transition point from steady state behavior to a fraction of oscillatory behavior. The number of beads in each experiment is between 50 and 150. (b) The mean period of the individual oscillatory beads. Averages are determined for 50 to 150 beads for each data point. (c) Time series for individual oscillating beads. The frequency distribution of the oscillating beads is shown in Fig. 6.3. The black line indicates the average of 71 beads while the colored lines represent typical behaviors of the oscillatory beads. (d) Time series for scattered beads that exhibit steady state behavior. 
a fraction of the particles were oscillatory. A typical time series of the oscillations is shown in Fig. 6.2(c). From Fig. 6.2(c), we can see that the oscillatory frequencies of the individual particles are quite different even with the same reaction mixture composition. This period difference has also been observed by the other groups [1, 12]. A histogram of the frequencies is shown in Fig. 6.3. The average frequencies for between 50 to 150 beads is shown in Fig. 6.2(b). As we can see in Fig. 6.3, an increase in bromate concentration shifts the oscillations to higher frequencies and decreases the frequency range.

The distribution of oscillatory frequency arises from variations in bead size and degree of ferroin loading $[12,14]$. Figure 6.4 shows the size variation of the beads. Ferroin loading is not homogeneous due to the inhomogeneity of the active ion exchange sites and the size of the beads [15]. It is known that the oscillation period can be affected by the concentration of ferroin in the BZ reaction [14]. In addition, different sizes of beads have different curvature that affects the exchange of species between the beads and the solution. Experimental observations indicate that larger beads are more excitable than smaller beads with the same concentration of ferroin [12].

\subsection{Experimental Results}

\subsubsection{Experimental Setup}

The collective behavior was studied by positioned the beads on the bottom of a petri dish to form a quasi-circular monolayer. The distance between nearest neighbor beads was less than one bead diameter within the group. The petri dish was filled with a thin layer of catalyst-free BZ solution. Color images were taken at fixed intervals with a digital camera (Insight IN1120) attached to an inverted microscope (Leica DM IRB). Some experiments were carried out with a digital camera (Infinity 1-3c) with a zoom lens (Computar 18-108/25 or Meiji MS-50D). The petri dish was illuminated from below with both configurations, and the images recorded the transmitted light through the beads. Images were recorded for 30 min after allowing the system to relax to its asymptotic behavior over a 20 min period. If wave activity was observed in a group of beads, the beads were then redistributed such 

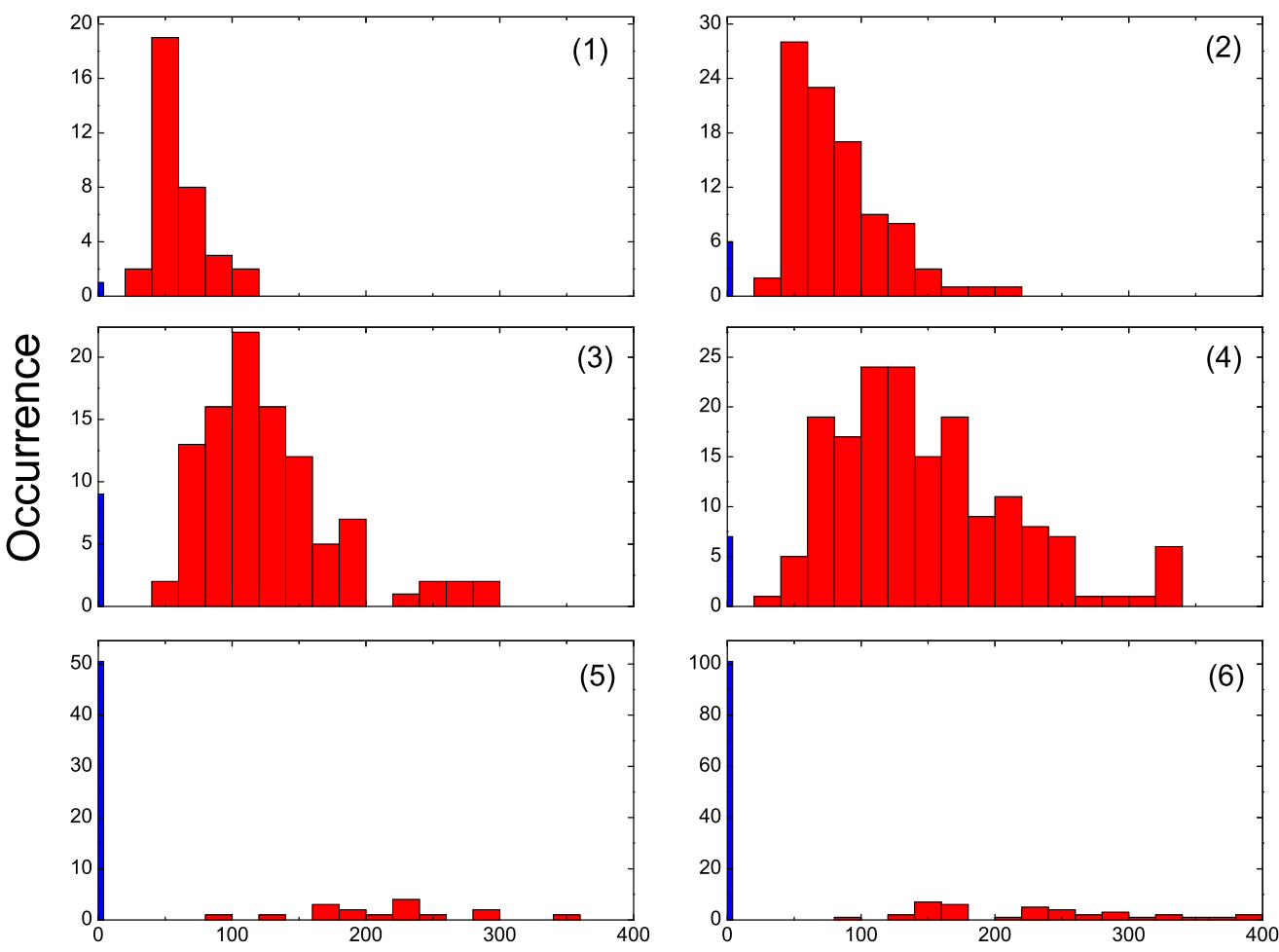

Oscillation Period (s)

Figure 6.3. Oscillatory period of independent catalytic particles. The solution concentrations are $[\mathrm{NaBr}]=0.10 \mathrm{M},[\mathrm{MA}]=0.20 \mathrm{M},\left[\mathrm{H}_{2} \mathrm{SO}_{4}\right]=0.60 \mathrm{M}$, and $\left[\mathrm{NaBrO}_{3}\right]=0.60 \mathrm{M}(1), 0.54$ $\mathrm{M}(2), 0.48 \mathrm{M}(3), 0.42 \mathrm{M}(4), 0.39 \mathrm{M}(5)$, and $0.36 \mathrm{M}$ (6). The beads are loaded with ferroin at a concentration of $1.7 \times 10^{-5} \mathrm{~mol} \mathrm{~g}^{-1}$. 


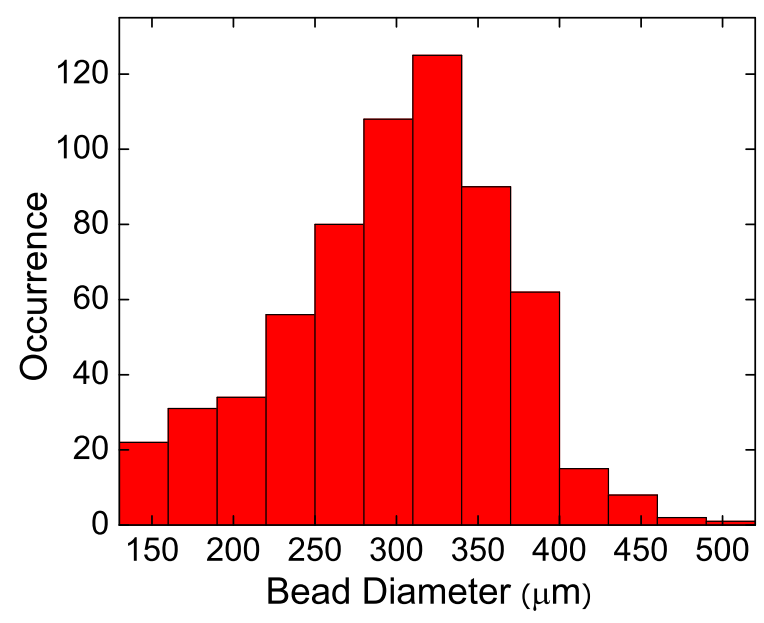

Figure 6.4. The size distribution of Bio-Rad analytical grade 50W-X (50-100 mesh) beads. The bead diameters are determined from images taken with a CCD camera. The beads are located by applying appropriate gray level thresholds and the areas (A) are obtained by counting the number of pixels for each bead. The bead diameters $(d)$ are calculated by $d=\frac{4 A}{\pi} \times C$, where $C$ is the calibration constant $\mu \mathrm{m}$ pixel $^{-1}$.

that they were separated from their neighboring beads by at least 5 bead diameters. The population was then monitored for an additional $30 \mathrm{~min}$ to ensure that the population did not contain any oscillatory beads. Independent experiments showed that beads do not interact when separated by 5 or more bead diameters.

Data analysis was carried out by monitoring the light intensity (greyscale) variation of the beads vs. time. The color to greyscale conversion was carried out by using the following empirical equation $[16,17]$ :

$$
\text { Greyscale }=0.299 \times \text { red }+0.587 \times \text { green }+0.114 \times \text { blue }
$$

Red, green and blue represent the corresponding color components for each pixel in the image ranging between 0 and 255. The resulting greyscale value represents the light intensity, which also ranges between 0 and 255 .

The images of the beads shown in Fig. 6.8 were enhanced by the following method. A sequence of $n$ images was taken at one frame per second. Wave images were acquired by subtracting image $i$ from image $i+3(i=1,2, \ldots$ n-3), with the subtraction carried out for the 
individual color components of each pixel. A background image is acquired by taking the darkest of each pixel in a sequence of images for wave segments or by selecting an image without wave activity for target wave patterns. The final image is obtained by adding two or three times the wave image to the background image.

Experiments with excitable and oscillatory beads were carried out with different concentrations of bromate and constant concentrations of malonic acid, bromide and sulfuric acid. A transition from steady state to oscillatory behavior occurred with the excitable beads when the size of the group was increased above a critical size. A synchronization process occurred with the oscillatory beads; however, the beads remained oscillatory. The details of the results shall be presented and discussed below.

\subsubsection{Results with Excitable System}

For a group of excitable beads, steady state or oscillatory behavior was exhibited by the group depending on its size, as shown in Fig. 6.5. Steady state behavior was observed in smaller groups. Oscillatory behavior was observed in large groups, with the oscillatory patterns either target waves or wave segments. A qualitative change in behavior for each bead in the oscillatory groups occurs, since an individual isolated bead exhibits steady state behavior. A transition region exists at an intermediate group size, in which a group with same number of beads has a probability of exhibiting steady state or oscillatory behavior. The three regions were observed at three different bromate concentrations. Two trends were observed when comparing the group behavior at different bromate concentrations. First, a larger group is required to show wave activity with lower bromate concentration. Second, the transition region is larger for lower bromate concentration. These trends can be clearly seen in Fig. 6.5.

The oscillation period of the waves was characterized by averaging the periods of the individual beads. The dependence of the period on bromate concentration for target waves and broken waves is shown in Fig. 6.6. For the same size of group, the period for the broken waves was always shorter than for the target waves. At each bromate concentration, the period of either target waves or broken waves displayed a large variation. The average

period of the target waves was essentially unaffected by the bromate concentration. The 


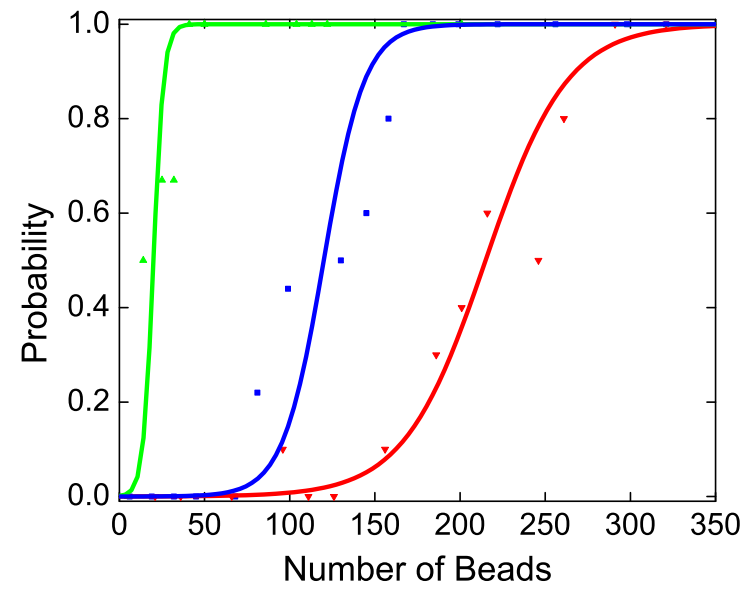

(a)

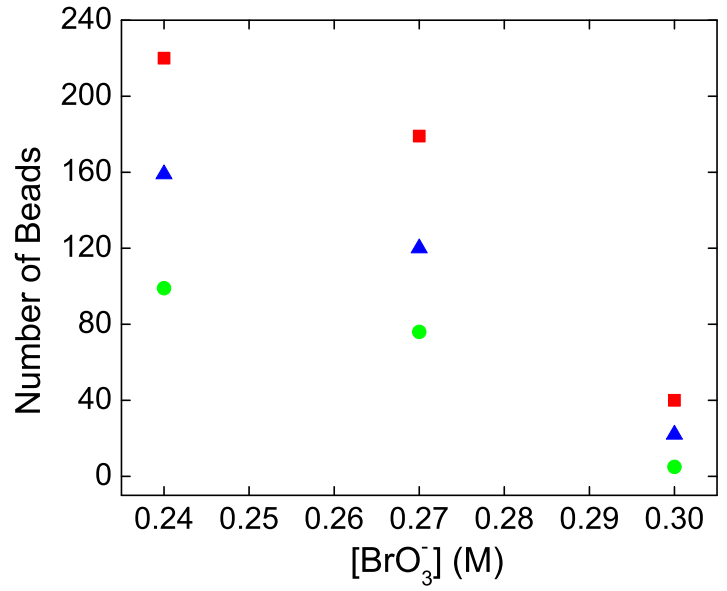

(b)

Figure 6.5. Spontaneous formation of wave activity from groups of steady state beads. The concentrations are $[\mathrm{NaBr}]=0.10 \mathrm{M},[\mathrm{MA}]=0.20 \mathrm{M},\left[\mathrm{H}_{2} \mathrm{SO}_{4}\right]=0.60 \mathrm{M},\left[\mathrm{NaBrO}_{3}\right]=0.33$ $\mathrm{M}$ (red), $\left[\mathrm{NaBrO}_{3}\right]=0.30 \mathrm{M}$ (blue), and $\left[\mathrm{NaBrO}_{3}\right]=0.27 \mathrm{M}$ (green). The beads are loaded with ferroin at a concentration of $1.7 \times 10^{-5} \mathrm{~mol} \mathrm{~g}^{-1}$. (a) Diagram showing wave initiation probability as a function of the number of beads in the group. The probability is defined by the number of experiments exhibiting wave behavior divided by the total number of experiments. Each point represents the probability determined from $\sim 8$ experiments. The variation in the group size is \pm 6 beads. (b) The green circles, red triangles and blue squares represent the beginning, center and end of the transition in panel (a). 


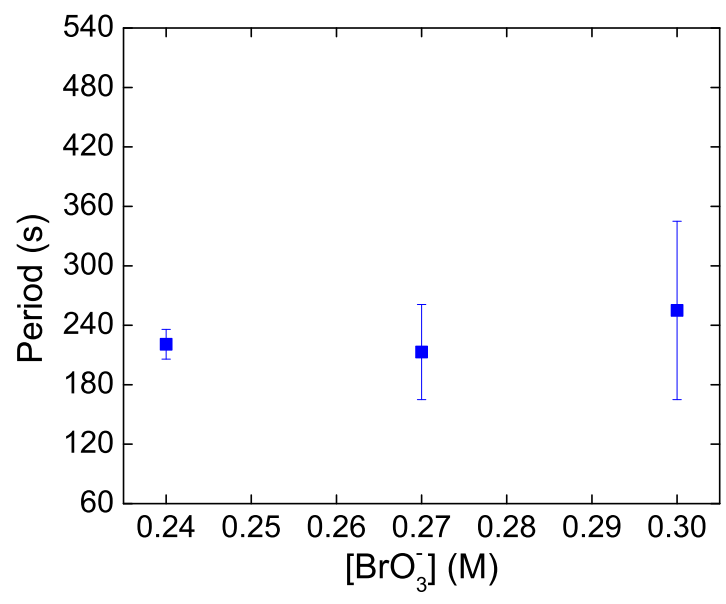

(a)

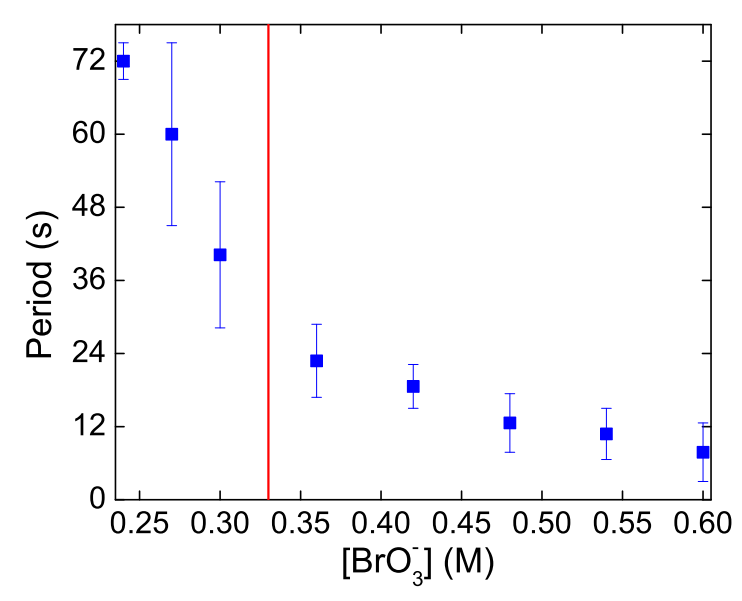

(b)

Figure 6.6. Dependence of wave period on bromate concentration. The concentrations are $[\mathrm{NaBr}]=0.10 \mathrm{M},[\mathrm{MA}]=0.20 \mathrm{M}$, and $\left[\mathrm{H}_{2} \mathrm{SO}_{4}\right]=0.60 \mathrm{M}$. The beads are loaded with ferroin at a concentration of $1.7 \times 10^{-5} \mathrm{~mol} \mathrm{~g}^{-1}$. (a) Dependence of target wave period on bromate concentration. No target waves were observed for bromate concentrations above 0.33 M. (b) Dependence of period of spirals on bromate concentration. The vertical red line represents the transition from steady state to oscillatory behavior for individual beads.

average period of the wave segments decreased with increasing bromate concentration.

\subsubsection{Features of Target Waves}

Target waves and broken waves were observed in large groups of excitable beads. Target waves appeared only in the excitable systems, while broken waves appeared in both the excitable and oscillatory systems. Target waves were more frequently observed than broken waves and accounted for $\sim 70 \%$ of the experiments displaying wave activity in the excitable systems. Target waves were generally initiated from one or more beads that were randomly located within a group. The initiation is indicated by a color change from red to blue, which corresponds to a change in the oxidation state of Fe from $2+$ to $3+$. After initiation, the wave propagated and covered the entire group of beads. Following the wave propagation, the beads remained in the reduced state until another wave was initiated. The initiation site or 


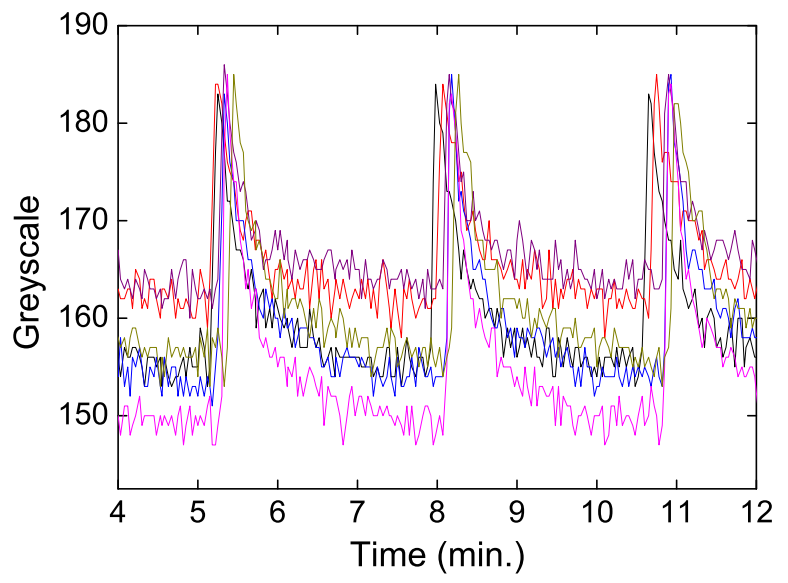

(a)

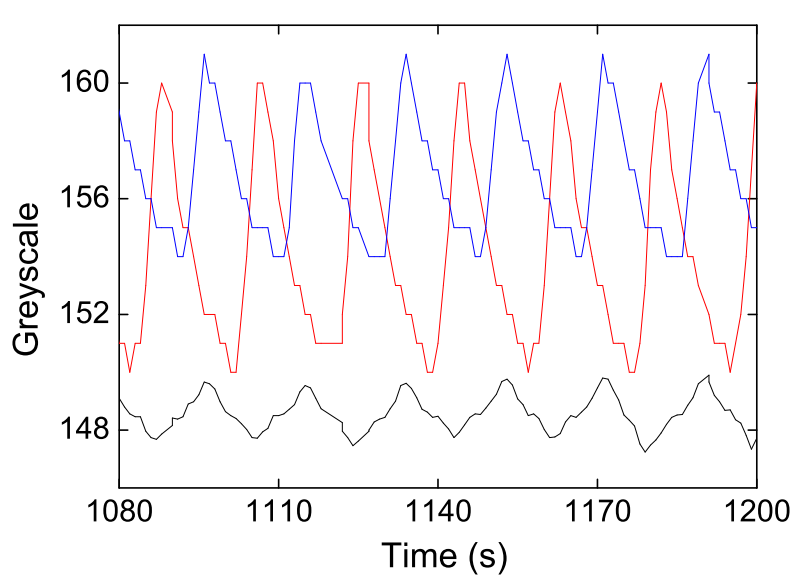

(b)

Figure 6.7. Typical time series for a target wave and a spiral wave propagating in a group of beads. The concentrations are $[\mathrm{NaBr}]=0.10 \mathrm{M},[\mathrm{MA}]=0.20 \mathrm{M},\left[\mathrm{H}_{2} \mathrm{SO}_{4}\right]=0.60 \mathrm{M}$, $\left[\mathrm{NaBrO}_{3}\right]=0.30 \mathrm{M}$ (panel (a)) and $0.54 \mathrm{M}$ (panel (b)). (a) Typical time series for target waves in excitable system. The curves represent sampling points that are equally spaced at $272 \mu \mathrm{m}$ intervals. The beads are placed on the bottom of the petri dish to form a circular monolayer and the distance between neighboring beads is less than one bead diameter. In order to compare the phase difference between different positions within a group, each curve is shifted to line up the first peak. (b) Typical time series for broken waves in oscillatory system. The total number of beads within the group is 200 . The black curve indicates the average over all beads.

sites may be from the same bead or beads; however, initiation may occur at different beads during each experiment. Occasionally broken waves collapsed into target waves. A typical time series was constructed by plotting the greyscale of a particular bead within a group as a function of time, as shown in Fig. 6.7. The top row of Fig. 6.8(a) shows sanpshots of target wave behavior. 

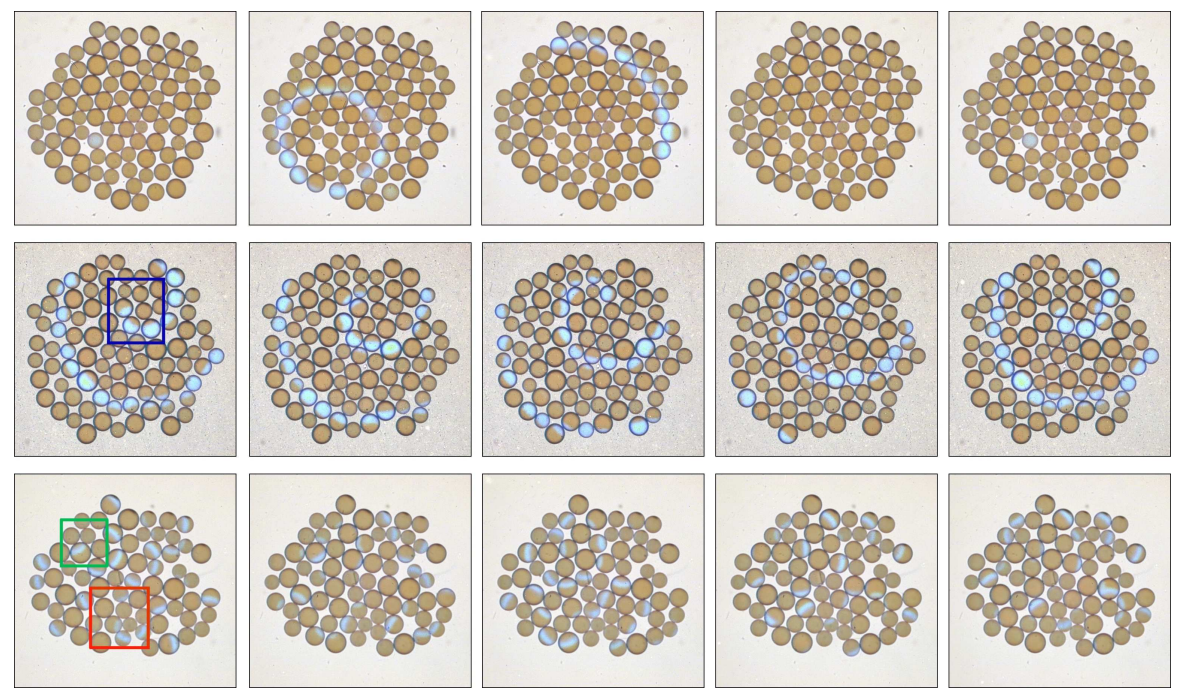

(a)
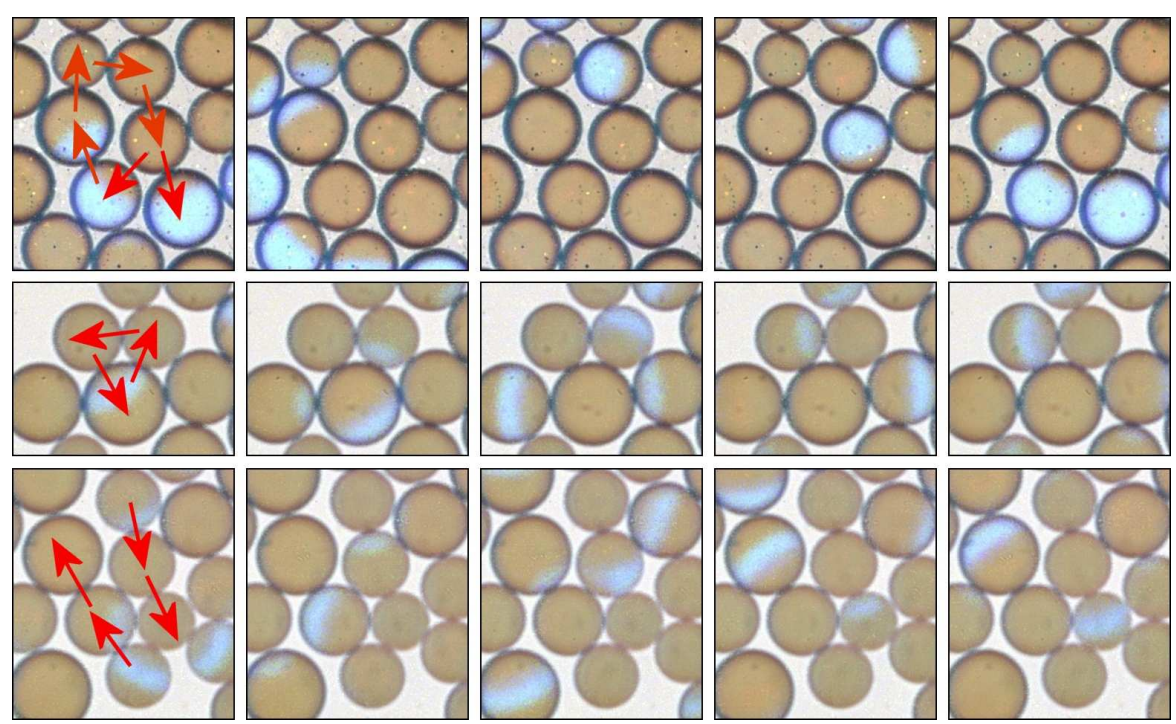

(b)

Figure 6.8. Snapshots of target wave behavior, and simple and complex broken waves. (a) Top, middle and bottom rows display time sequences of a target wave, a spiral wave and complex behavior of wave segments. The concentrations are $[\mathrm{NaBr}]=0.10 \mathrm{M},[\mathrm{MA}]=0.20$ $\mathrm{M}$, and $\left[\mathrm{H}_{2} \mathrm{SO}_{4}\right]=0.60 \mathrm{M}$ for all three waves, with $\left[\mathrm{BrO}_{3}\right]=0.30 \mathrm{M}$ (top), $0.42 \mathrm{M}$ (middle) and $0.47 \mathrm{M}$ (bottom). Image intervals are $12 \mathrm{~s}, 12 \mathrm{~s}, 65 \mathrm{~s}$ and $65 \mathrm{~s}$ for top row, $5 \mathrm{~s}$ for the middle row, and $4 \mathrm{~s}$ for the bottom row. (b) Close-up view of the details of the blue (top row), green (middle row) and red squares (bottom row) in image (a). Wave propagation directions are indicated by the arrows. 


\subsubsection{Features of Broken Waves}

Broken waves appeared in both excitable and oscillatory systems and they commonly exhibited stable spatiotemporal patterns. The waves were typically initiated from one or more centers. Figure 6.8(a) shows an example of broken wave activity. The centers take the form of a fast reentrant wave circulating [18] around a hole in the bead packing, as shown in Fig. 6.8(b). At higher bromate concentrations, waves on neighboring beads traveling on opposite directions were also observed, as shown in the bottom row of Fig. 6.8(b).

Insights into the complexity of the systems can be gained by considering each as a network of beads connected to their respective reentrant center [19]. We define the network distance of a bead as the number of intermediate beads a wave must travel through in order to reach that bead. Figure 6.9 shows such a map of the network distance. For lower bromate concentrations, the network distance is related to the Euclidean distance of a bead from the center, as shown in Fig. 6.9(a). For higher bromate concentrations, however, the network distance is more complex, as shown in Fig. 6.9(b).

Analysis of broken waves in oscillatory systems shows that all of the beads are synchronized in frequency but not in phase. The average intensity of all the beads in a group oscillates at a much smaller amplitude, as shown in Fig. 6.7(b).

\subsection{Simulation Results}

\subsubsection{ZBKE Model of the Belousov-Zhabotinsky Reaction}

Simulation studies of groups of beads were carried out with the ZBKE model, which was developed by Zhabotinsky, Buchholtz, Kiyatkin and Epstein in 1993 [20]. The ZBKE model gives a broader frequency distribution of oscillatory behavior with variation of parameters than the Oregonator model. The model reaction scheme is shown in Table 6.1.

In the reaction scheme, red is the reduced form of the catalyst, $\mathrm{Fe}(\mathrm{phen})_{3}^{2+}$, and $o x$ is the oxidized form, Fe(phen $)_{3}^{3+}$. Reactions (RA-1) and (RA-2) are assumed to be sufficiently fast that other reactions involving $\mathrm{HOBr}$ and $\mathrm{Br}_{2}$ are negligible. $\mathrm{CBr}_{2}(\mathrm{COOH})_{2}$, $\mathrm{CHOH}(\mathrm{COOH})_{2}$ and $\mathrm{CO}(\mathrm{COOH})_{2}$ are considered to be final products. The equilibrium of 


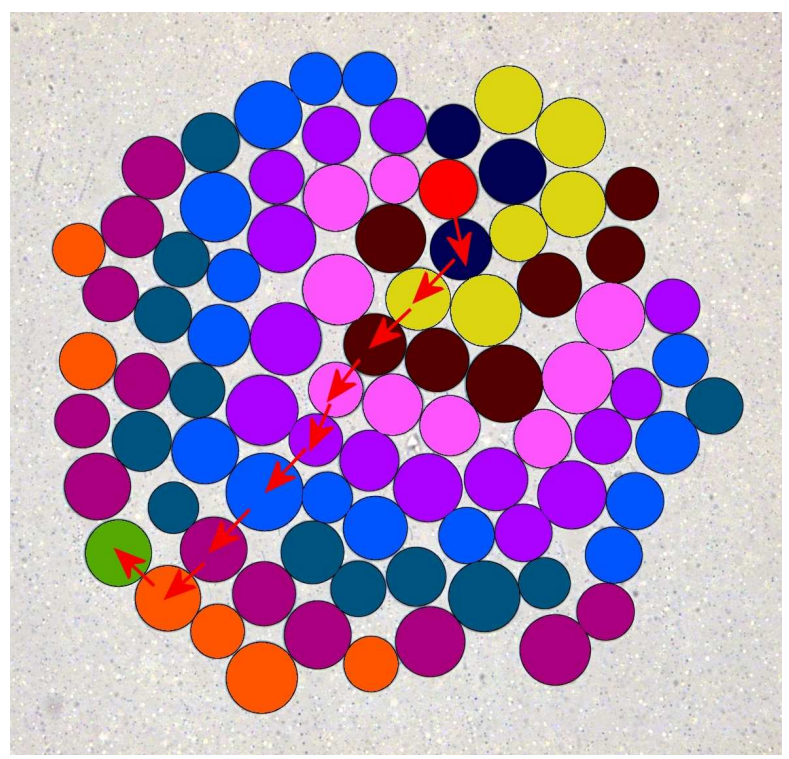

(a)

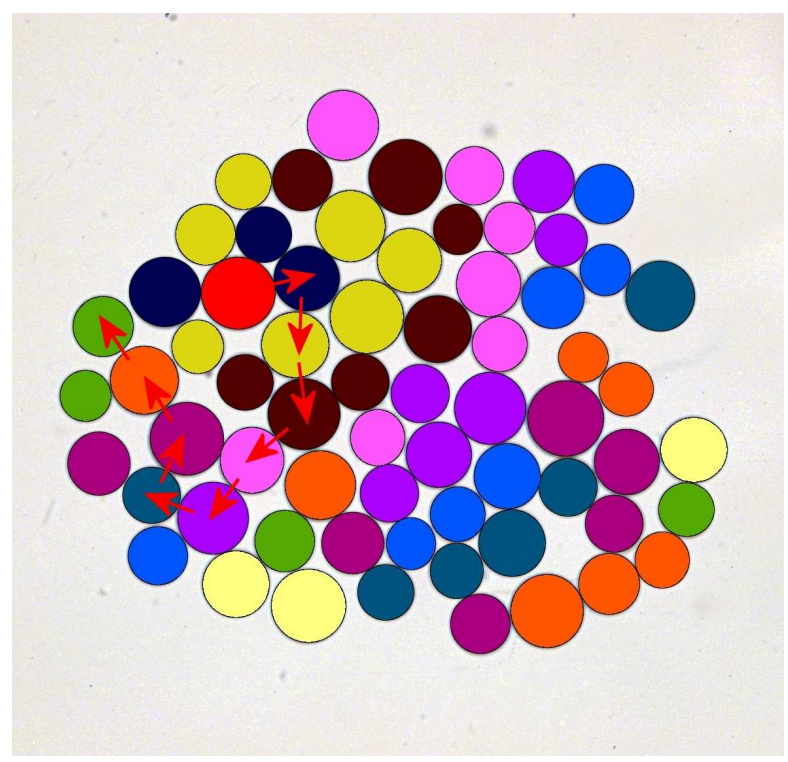

(b)

Figure 6.9. Sequence of bead initiations of a simple and complex broken wave. The arrows indicate how the wave starts from the center bead and reaches the group edge. The concentrations are $[\mathrm{NaBr}]=0.10 \mathrm{M},[\mathrm{MA}]=0.20 \mathrm{M}$, and $\left[\mathrm{H}_{2} \mathrm{SO}_{4}\right]=0.60 \mathrm{M},\left[\mathrm{BrO}_{3}\right]=0.42 \mathrm{M}$ (panel (a)) and $0.45 \mathrm{M}$ (panel (b)). (a) Order of wave propagation through the beads. (b) Order of propagation through the beads for a complex broken wave. 
Table 6.1. The reaction scheme for the ZBKE model

\begin{tabular}{|c|c|}
\hline Equation Number & Reaction \\
\hline $\mathrm{H}^{+}+\mathrm{Br}^{-}+\mathrm{HOBr} \rightleftharpoons \mathrm{Br}_{2}+\mathrm{H}_{2} \mathrm{O}$ & $(\mathrm{R} 1)$ \\
\hline $\mathrm{H}^{+}+\mathrm{Br}^{-}+\mathrm{HBrO}_{2} \rightleftharpoons 2 \mathrm{HOBr}$ & $(\mathrm{R} 2)$ \\
\hline $\mathrm{H}^{+}+\mathrm{Br}^{-}+\mathrm{HBrO}_{3} \rightleftharpoons \mathrm{HBrO}_{2}+\mathrm{HOBr}$ & (R3) \\
\hline $2 \mathrm{HBrO}_{2} \rightleftharpoons \mathrm{HOBr}+\mathrm{HBrO}_{3}$ & $(\mathrm{R} 4 \mathrm{a})$ \\
\hline $\mathrm{H}^{+}+\mathrm{HBrO}_{2} \rightleftharpoons 2 \mathrm{H}_{2} \mathrm{BrO}_{2}^{+}$ & $(\mathrm{R} 4 \mathrm{~b})$ \\
\hline $\mathrm{HBrO}_{2}+\mathrm{H}_{2} \mathrm{BrO}_{2}^{+} \rightleftharpoons \mathrm{HOBr}+\mathrm{HBrO}_{3}+\mathrm{H}^{+}$ & $(\mathrm{R} 4 \mathrm{c})$ \\
\hline $\mathrm{H}^{+}+\mathrm{BrO}_{3}^{-} \rightleftharpoons \mathrm{HBrO}_{3}$ & $(\mathrm{R} 5 \mathrm{a})$ \\
\hline $\mathrm{H}^{+}+\mathrm{HBrO}_{3}+\mathrm{HBrO}_{2} \rightleftharpoons \mathrm{HBrO}_{2}^{+}+\mathrm{BrO}_{2} \cdot+\mathrm{H}_{2} \mathrm{O}$ & $(\mathrm{R} 5 \mathrm{~b})$ \\
\hline $\mathrm{BrO}_{2} \cdot+\mathrm{H}^{+} \rightleftharpoons \mathrm{HBrO}_{2}^{+}$ & $(\mathrm{R} 5 \mathrm{c})$ \\
\hline $\mathrm{red}+\mathrm{HBrO}_{2}^{+} \rightleftharpoons \mathrm{ox}+\mathrm{HBrO}_{2}$ & $(\mathrm{R} 6)$ \\
\hline $\mathrm{Ox}+\mathrm{CHBr}(\mathrm{COOH})_{2} \rightleftharpoons \mathrm{red}+\mathrm{CBr}(\mathrm{COOH})_{2} \cdot+\mathrm{H}^{+}$ & $(\mathrm{R} 7)$ \\
\hline $\mathrm{H}_{2} \mathrm{O}+\mathrm{CBr}(\mathrm{COOH})_{2} \cdot \rightarrow \mathrm{H}^{+}+\mathrm{Br}^{-}+\mathrm{COH}(\mathrm{COOH})_{2}$ & $(\mathrm{R} 8)$ \\
\hline $\mathrm{H}_{2} \mathrm{O}+\mathrm{CHBr}(\mathrm{COOH})_{2} \rightarrow \mathrm{CHOH}(\mathrm{COOH})_{2}+\mathrm{H}^{+}+\mathrm{Br}^{-}$ & $(\mathrm{R} 9)$ \\
\hline $2 \mathrm{COH}(\mathrm{COOH})_{2} \cdot \rightarrow \mathrm{CHOH}(\mathrm{COOH})_{2}+\mathrm{CO}(\mathrm{COOH})_{2}$ & $(\mathrm{R} 10)$ \\
\hline $\mathrm{COH}(\mathrm{COOH})_{2} \cdot+\mathrm{CBr}(\mathrm{COOH})_{2} \cdot \rightarrow \mathrm{CHBr}(\mathrm{COOH})_{2}+\mathrm{CO}(\mathrm{COOH})_{2}$ & $(\mathrm{R} 11)$ \\
\hline $\mathrm{HOBr}+\mathrm{CHBr}(\mathrm{COOH})_{2} \rightarrow \mathrm{CBr}_{2}(\mathrm{COOH})_{2}+\mathrm{H}_{2} \mathrm{O}$ & $(\mathrm{RA}-1)$ \\
\hline $\mathrm{Br}_{2}+\mathrm{CHBr}(\mathrm{COOH})_{2} \rightarrow \mathrm{CBr}_{2}(\mathrm{COOH})_{2}+\mathrm{H}^{+}+\mathrm{Br}^{-}$ & $(\mathrm{RA}-2)$ \\
\hline
\end{tabular}


reaction (R5c) is considered to be shifted almost completely to the right in highly acidic media. The corresponding mathematical model can be derived as shown below.

$$
\begin{aligned}
\frac{d X}{d t} & =-k_{2} h_{0} X Y+k_{3} h_{0} X Y-2 k_{4}^{*} X^{2}-k_{5} h_{0} A X+k_{-5} U^{2}+k_{6} U(C-Z)-k_{-6} X Z \\
\frac{d Y}{d t} & =-k_{2} h_{0} X Y-k_{3} h_{0} A Y+k_{8}^{\prime} R_{1}+k_{9} B \\
\frac{d U}{d t} & =2 k_{5} h_{0} A X-2 k_{-5} U^{2}-k_{6} U(C-Z)+k_{-6} X Z \\
\frac{d Z}{d t} & =k_{6} U(C-Z)-k_{-6} X Z-k_{7} B Z+k_{-7} h_{0} R_{1}(C-Z) \\
\frac{d R_{1}}{d t} & =k_{7} B Z-k_{-7} h_{0} R_{1}(C-Z)-k_{8}^{\prime} R_{1}-k_{11} R_{1} R_{2} \\
\frac{d R_{2}}{d t} & =k_{8}^{\prime} R_{1}-2 k_{10} R_{2}^{2}-k_{11} R_{1} R_{2}
\end{aligned}
$$

Here, $X=\left[\mathrm{HBrO}_{2}\right], Y=\left[\mathrm{Br}^{-}\right], U=\left[\mathrm{HBrO}_{2}^{+}\right], Z=[o x], R_{1}=\left[\mathrm{CBr}(\mathrm{COOH})_{2} \cdot\right], R_{2}=$ $\left[\mathrm{COH}(\mathrm{COOH})_{2} \cdot\right], A=\left[\mathrm{HBrO}_{3}\right]=\mathrm{h}_{0}\left[\mathrm{NaBrO}_{3}\right]_{0} /\left(0.2+\mathrm{h}_{0}\right), B=\left[\mathrm{CHBr}(\mathrm{COOH})_{2}\right], C=Z+$ $[\mathrm{red}], \mathrm{h}_{0}=$ Hammet acidity function, $k_{4}^{*}=k_{4}\left(1+0.87 h_{0}\right)$, and $k_{-5}$ represents $\left(k_{-5 b} k_{-5 c}\right) /\left(k_{5 c} h_{0}\right)$.

Using the quasi-steady-state approximation for $R_{2}$ and assuming that radical recombination reactions $\mathrm{R} 10$ and $\mathrm{R} 11$ are fast, we can obtain equation for $\mathrm{R}_{1}$.

$$
d R_{1} / d t=k_{7} B Z-k_{-7} h_{0} R_{1}(C-Z)-\frac{k_{8}^{\prime} R_{1}}{q\left(R_{1}\right)}
$$

where $\frac{1}{q\left(R_{1}\right)}=1-\frac{k_{11}^{2} R_{1}}{4 k_{8}^{\prime} k_{10}}\left(1-\left(1+\frac{8 k_{8}^{\prime} k_{10}}{k_{11}^{2} R_{1}}\right)^{1 / 2}\right)$. Using an Oregonator approach, a parameter $q$ is introduced to replace $q\left(R_{1}\right)$. This can be arbitrarily varied from 0.5 to 1.0 (reaction R10 is much faster than R11). The rate constant $k_{8}$ is defined as $\frac{k_{8}^{\prime}}{q}$.

By assuming the concentrations of bromomalonyl radical and bromide ion are fast variables, a three variable model can be developed. 


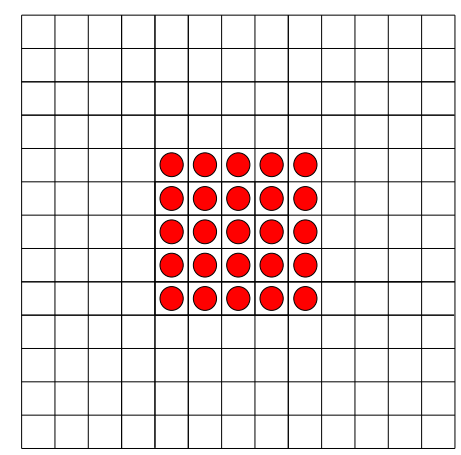

(a)

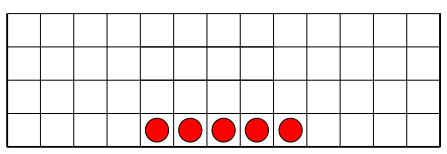

(b)

Figure 6.10. Schematic drawing of simulation model for coupled bead system. (a) Top view of the bead placement. (b) Side view of the bead placement. [21]

$$
\begin{aligned}
\frac{d X}{d t} & =-k_{2} h_{o} X Y+k_{3} h_{0} A Y+q \frac{k_{7} k_{8} B Z}{k_{-7} h_{0}(C-Z)+k_{8}}+k_{9} B \\
\frac{d Y}{d t} & =-k_{2} h_{0} X_{i} Y_{i}-k_{3} h_{0} X Y-k_{3} h_{0} A Y+q \frac{k_{7} k_{8} B Z}{k_{-7} h_{0}(C-Z)+k_{8}}+k_{9} B \\
\frac{d Z}{d t} & =k_{6} U(C-Z)-k_{-6} X Z-\frac{k_{7} k_{8} B Z}{k_{8}+k_{-7} h_{0}(C-Z)}
\end{aligned}
$$

\subsubsection{Simulation Model for the Spatially Coupled Bead System}

The simulation model is based on a square lattice of cells. The catalyst-loaded beads are placed into adjacent cells and are in direct contact with each other, as shown in Fig. 6.10. A monolayer is formed, with the beads locally coupled by diffusion according to a six-point Laplacian with no-flux boundary conditions. Exchange of activator $\left(\mathrm{HBrO}_{2}\right)$ and inhibitor $\left(\mathrm{Br}^{-}\right)$with the solution cells also occurs as a diffusion process. Studies of homogeneously and heterogeneously ferroin-loaded beads were carried out. The parameters of the model are listed in Table 6.2.

The rate equations for the activator, inhibitor and catalyst for particle cell $i$ are given 
Table 6.2. Rate constants for the numerical simulations

\begin{tabular}{|c|c||c|c|}
\hline Rate constants & Values & Rate constants & Values \\
\hline$k_{2}\left(M^{-2} s^{-1}\right)$ & $4.81 \times 10^{6}$ & $k_{5}\left(M^{-1} s^{-1}\right)$ & $4.2 \times 10^{6}$ \\
\hline$k_{-2}\left(M^{-1} s^{-1}\right)$ & 0 & $k_{6}\left(M^{-1} s^{-1}\right)$ & $1.66 \times 10^{7}$ \\
\hline$k_{3}\left(M^{-2} s^{-1}\right)$ & 2.0 & $k_{-6}\left(M^{-1} s^{-1}\right)$ & 0.3 \\
\hline$k_{-3}\left(M^{-1} s^{-1}\right)$ & 0 & $k_{8} / k_{-7}\left(M^{2}\right)$ & $3 \times 10^{-6}$ \\
\hline$k_{4}\left(M^{-1} s^{-1}\right)$ & $8.6 \times 10^{3}$ & $k_{7} k_{8} / k_{-7}\left(M s^{-1}\right)$ & $5 \times 10^{-7}$ \\
\hline$k_{-4}\left(M^{-1} s^{-1}\right)$ & 0 & $k_{9}\left(s^{-1}\right)$ & $3.3 \times 10^{-6}$ \\
\hline$k_{5}\left(M^{-1} s^{-1}\right)$ & 16.03 & & \\
\hline
\end{tabular}

by Eqs 6.7 .

$$
\begin{aligned}
\frac{d X i}{d t} & =-k_{2} h_{0} X i Y i+k_{3} h_{0} A Y i-2 k_{4} X_{i}^{2}+q \frac{k_{7} k_{8} B Z i}{k_{-7} h_{0}\left(C_{i}-Z_{i}\right)+k_{8}}+k_{9} B+D \nabla^{2} X_{i} \\
\frac{d Y_{i}}{d t} & =-k_{2} h_{0} X_{i} Y_{i}-k_{3} h_{0} A Y_{i}+q \frac{k_{7} k_{8} B Z i}{k_{-7} h_{0}\left(C_{i}-Z_{i}\right)+k_{8}}+k_{9} B+D \nabla^{2} Y_{i} \\
\frac{d Z_{i}}{d t} & =k_{6} U_{s s}\left(C_{i}-Z_{i}\right)-k_{-6} X_{i} Z_{i}-\frac{k_{7} k_{8} B Z_{i}}{k_{8}+k_{-7} h_{0}\left(C_{i}-Z_{i}\right)}
\end{aligned}
$$

The rate equations for activator and inhibitor for solution cell $i$ are given by Eqs 6.8.

$$
\begin{aligned}
& \frac{d X i}{d t}=-k_{2} h_{0} X i Y i-k_{3} h_{0} A Y i+k_{9} B+D \nabla^{2} X_{i} \\
& \frac{d Y_{i}}{d t}=-k_{2} h_{0} X_{i} Y_{i}-k_{3} h_{0} A Y_{i}+k_{9} B+D \nabla^{2} Y_{i}
\end{aligned}
$$

The system is integrated using an Euler scheme with time step $d t=0.001 \mathrm{~s}$, diffusion coefficient $D=2.5 \times 10^{-3} \mathrm{~mm}^{2} \mathrm{~s}^{-1}$, and a spatial grid size equal to the mean particle size of $0.3 \mathrm{~mm}$. The typical $3 \mathrm{D}$ grid size is $22 \times 22 \times 4$.

\subsubsection{Simulation Results}

Quorum sensing type transitions were found in the ZBKE particle model, as shown in Fig. 6.11. Groups of homogeneously loaded beads, with all beads having the same concentration of ferroin, displayed a sharp transition from steady state to wave behavior on increasing the 


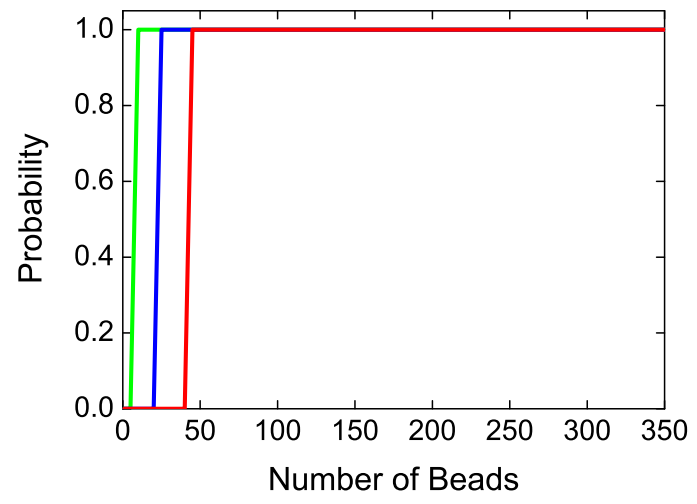

(a)

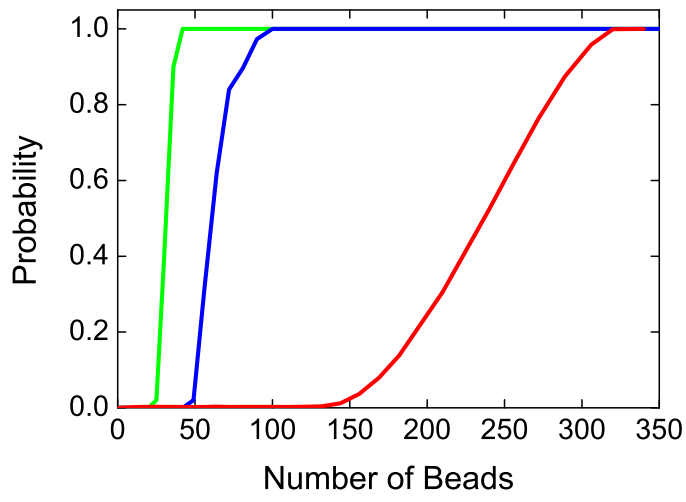

(b)

Figure 6.11. Transition from steady state to wave behavior as a function of group size. The parameters are $B=0.2 \mathrm{M}, h_{0}=0.73 \mathrm{M}, q=0.72, k_{2}=4.86 \times 10^{6}, k_{5}=16.03,[A]=0.2430$ M (green), 0.2425 M (blue) and 0.2420 M (red). (a) Results of homogeneous beads, with $C$ $=0.0053 \mathrm{M}$. (b) Results of heterogeneous beads, with $C_{\text {mean }}=0.0053 \mathrm{M}$ and $C_{\sigma}=0.0006 \mathrm{M}$. $[22]$

number of beads. Groups of heterogeneously loaded beads displayed a gradual change in behavior, similar to that observed in the experiments.

An analysis of the concentrations of the activator and inhibitor revealed that the quorum sensing transition occurring in the spatially distributed system is not strictly due to the accumulation of the activator. The concentration of activator in the vicinity of the pacemaker bead actually decreases when the group size increases, as shown in Fig. 6.12. Instead, the concentration of inhibitor increases as the group size increases. Further analysis suggests that the transition is associated with a decrease in the loss of activator to the solution.

\subsection{Conclusion}

Experiments carried out with a BZ bead system revealed a quorum sensing type transition in groups of excitable beads. The transition occurs from the excitable state to a spatiotemporal oscillatory state when the group size is increased above a threshold value. The oscillatory 


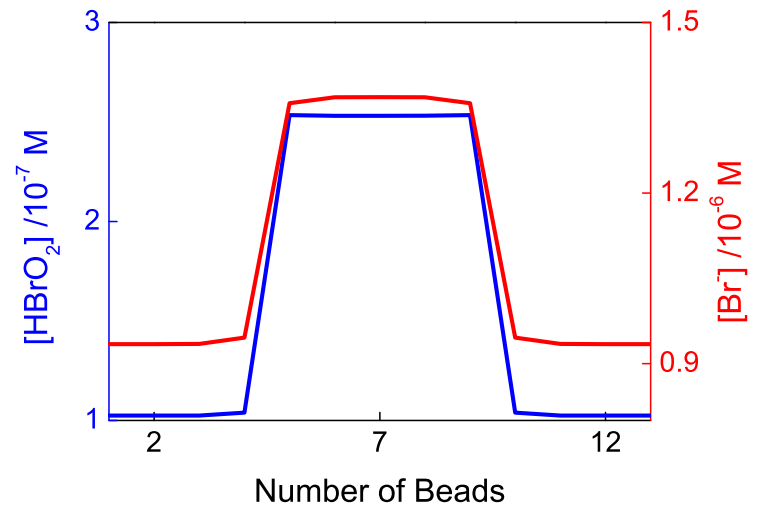

(a)

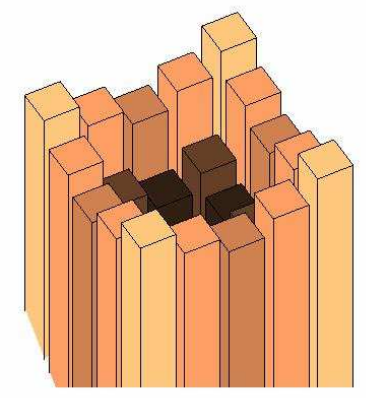

(c)

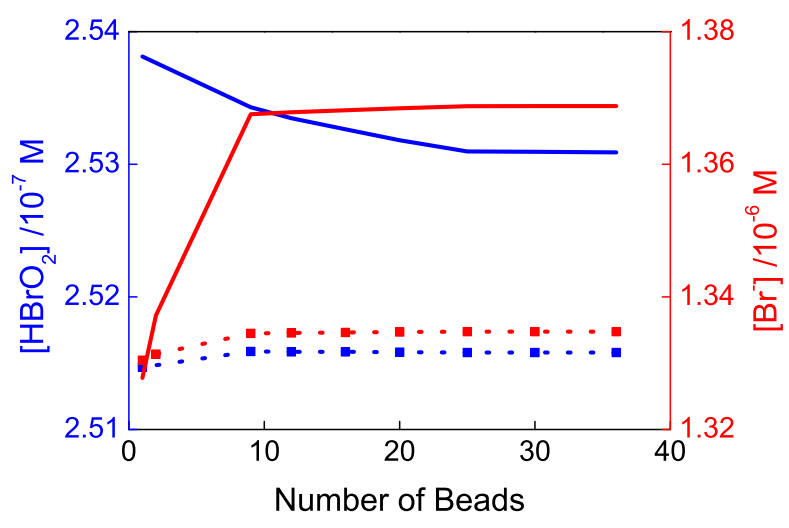

(b)

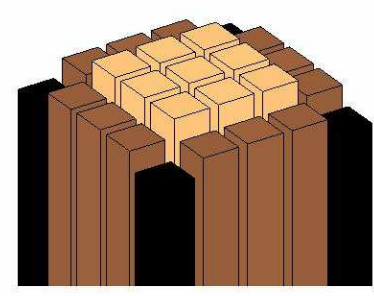

(d)

Figure 6.12. Concentrations of $\mathrm{Br}^{-}$and $\mathrm{HBrO}_{2}$ as a function of the number of beads. (a) Concentration profile of $\mathrm{Br}^{-}$(red) and $\mathrm{HBrO}_{2}$ (blue) across a group of beads. (b) Dependence of the concentrations of $\mathrm{Br}^{-}$and $\mathrm{HBrO}_{2}$ on number of beads. Solid lines represent the concentration near the center bead and dashed lines represent the concentration in the solution directly above this bead. (c) Three dimensional view of the concentration of $\mathrm{HBrO}_{2}$ of the beads in the group. (d) Three dimensional view of the concentration of $\mathrm{Br}^{-}$of the beads in the group. [22] 
behavior in a group of beads occurred as either target or broken wave patterns across the group. Numerical simulations with the ZBKE model suggest that the transition is due to a decrease in the loss of activator from the beads to the solution. 


\section{Bibliography}

[1] R. Toth, A. F. Taylor, and M. R. Tinsley, "Collective behavior of a population of chemically coupled oscillators," J. Phys. Chem. B 110, 10170-10176 (2006).

Online Version 98, 100, 102, 133

[2] A. T. Winfree, "The prehistory of the Belousov-Zhabotinsky oscillator," J. Chem. Educ. 61, 661-663 (1984).

Online Version 98

[3] R. Field and M. Burger, eds., Oscillations and traveling waves in chemical systems (John Wiley \& Sons, Princeton, NJ, 1985). 98

[4] I. R. Epstein and K. Showalter, "Nonlinear chemical dynamics: Oscillations, patterns, and chaos," J. Phys. Chem. 100, 13132-13147 (1996).

Online Version 98

[5] R. J. Field, E. Körös, and R. M. Noyes, "Oscillations in chemical systems. II. Thorough analysis of temporal oscillation in the bromate-cerium-malonic acid system," J. Am. Chem. Soc. 94, 8649-8664 (1972).

Online Version 99, 100

[6] B. P. Belousov, "A periodic reaction and its mechanism," in "Ref. Radiats. Med., 1958, 145," (1959). 100

[7] A. M. Zhabotinsky, "Periodic process of the oxidation of malonic acid in solution (study of kinetics of Belousov's reaction)," Biofizika 9, 306-311 (1964). 100 
[8] T. Sakurai, E. Mihaliuk, F. Chirila, and K. Showalter, "Design and control of wave propagation patterns in excitable media," Science 296, 2009-2012 (2002).

Online Version 100

[9] R. Yoshida, T. Takahashi, T. Yamaguchi, and H. Ichijo, "Self-oscillating gel," J. Am. Chem. Soc. 118, 5134-5135 (1996).

Online Version 54, 100

[10] J. Maselko and K. Showalter, "Chemical waves on spherical surfaces," Nature 339, 609-611 (1989).

Online Version 100

[11] J. Maselko and K. Showalter, "Chemical waves in inhomogeneous excitable media," Physica D 49, 21-32 (1991).

Online Version 100

[12] K. Yoshikawa, R. Aihara, and K. Agladze, "Size-dependent Belousov-Zhabotinsky oscillation in small beads," J. Phys. Chem. A 102, 7649-7652 (1998).

Online Version 100, 102

[13] R. J. Field and R. M. Noyes, "Oscillations in chemical systems. IV. Limit cycle behavior in a model of a real chemical reaction," J. Chem. Phys. 60, 1877-1884 (1974).

Online Version 100

[14] J. Wang, J. Zhao, Y. Chen, Q. Gao, and Y. Wang, "Coexistence of two bifurcation regimes in a closed ferroin-catalyzed Belousov-Zhabotinsky reaction," J. Phys. Chem. A 109, $1374-1381(2005)$.

Online Version 102

[15] E. Högfeldt, "Ion-exchange equilibria on single beads," Science 128, 1435-1436 (1958). Online Version 102

[16] C. Poynton, Digital video and HDTV algorithms and interfaces (Morgan Kaufmann, San Francisco, USA, 2003).

Online Version 104 
[17] W. Wharton and D. Howorth, Principles of Television Reception (Pitman Publishing, 1971). 104

[18] J. E. Strang and P. Osborn, "Wave patterns in frequency-entrained oscillator lattices," Phys. Rev. E 72, 056137 (2005).

Online Version 110, 139

[19] H. Kori and A. S. Mikhailov, "Entrainment of randomly coupled oscillator networks by a pacemaker," Phys. Rev. Lett. 93, 254101 (2004).

Online Version 110

[20] A. M. Zhabotinsky, F. Buchholtz, A. B. Kiyatkin, and I. R. Epstein, "Oscillations and waves in metal-ion-catalyzed bromate oscillating reactions in highly oxidized states," J. Phys. Chem. 97, 1518-7584 (1993).

Online Version 110, 130, 137, 140

[21] M. Tinsley, A. F. Taylor, Z. Huang, F. Wang, and K. Showalter, "Synchronization in collections of excitable and oscillatory catalytic particles," submitted. 114

[22] M. Tinsley, A. F. Taylor, Z. Huang, and K. Showalter, "Spatiotemporal dynamic quorum sensing in groups of excitable particles," submitted. 116, 117 


\section{Chapter 7}

\section{Collective Behavior of Globally Coupled Oscillators}

In Chapter 6, we discussed the emergent behavior in a collection of excitable and oscillatory beads coupled locally through diffusion. In this chapter, we will discuss the collective behavior when beads are globally coupled.

\subsection{Introduction}

Synchronization is found in many different types of systems, from coupled pendula to flashing fireflies. Synchronization of rhythmic activity is often of vital importance to the proper functioning of biological systems $[1,2,3]$. Coupling, external forcing, and applied noise may lead to the synchronization of dynamical systems [4]. In the 1980s, Kuramoto mathematically formalized a mechanism for the synchronization of a large number of phase oscillators through global coupling via a common (mean) field [5]. By varying the coupling strength, a collective rhythm emerges when the coupling strength is above a critical threshold. A number of synchronized systems fall into this category, such as suspended nanodroplets of chemical oscillators [6, 7], populations of E. Coli cells [8], and stirred suspensions of the cellular slime mold Dictyostelium discoidium [9]. The first experimental laboratory example of Kuramoto synchronization was found in a system of coupled electrochemical oscillators [10].

Here we use an experimental system of discrete chemical oscillators with well defined ki- 
netics to study synchronization phenomena in large populations of oscillators. By varying the number density of the oscillators, two distinct types of transitions were observed at different chemical exchange rates. At low chemical exchange rates, a Kuramoto type synchronization transition, a gradual rhythm alignment and synchronization of the oscillators, was observed. At high chemical exchange rates, a quorum sensing type transition, a sharp transition from steady state to synchronized oscillatory behavior, was observed. This type of quorum sensing transition has been observed in suspension of yeast cells [11, 12, 13]. Experimental variation of the chemical exchange rate was realized by changing the stirring rate. The relationship between the chemical exchange rate and the stirring rate is explained in the experimental section. The oxidation state of the ferroin was photographically recorded to characterize the transitions. The method of the image analysis is presented in the experimental section. Experimental observations of quorum sensing and Kuramoto synchronization and a numerical analysis are presented below.

\subsection{Experimental setup}

\subsubsection{Bead Preparation and Oscillation Properties}

The ferroin-loaded cation exchange beads, DOWEX 50WX4-200 (200 mesh), were prepared as described in Chapter 6. The catalyst concentration of the loaded beads was $1.7 \times 10^{-5} \mathrm{~mol} \mathrm{~g}^{-1}$. The number density $n$ of particles is estimated from Eq. 7.1.

$$
n=N / V_{s}=\frac{V_{p}}{\bar{V} V_{s}}
$$

where $N$ is the total number of particles, $V_{p}$ is the volume occupied by wet particles in solution, which is calculated as $1 / 5$ of the volume displacement of dry particles to account for the $80 \%$ solution absorption of the particles. $V_{s}$ is the total volume of the solution and $\bar{V}$ is the average volume of a particle, which is calculated by using $\bar{d}=120 \mu \mathrm{m}$. When a mass of $0.2 \mathrm{~g}$ of dry beads is added to $10 \mathrm{ml}$ of solution, the volume displacement is $0.17 \mathrm{ml}$ and the number of beads $N$ is $1.2 \times 10^{5}$ and $n=1.2 \times 10^{4} \mathrm{ml}^{-1}$.

The typical size distribution of the beads used in the experiment is shown in Fig. 7.1(a), 


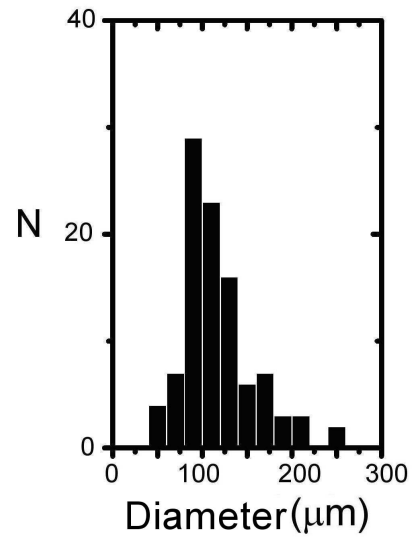

(a)

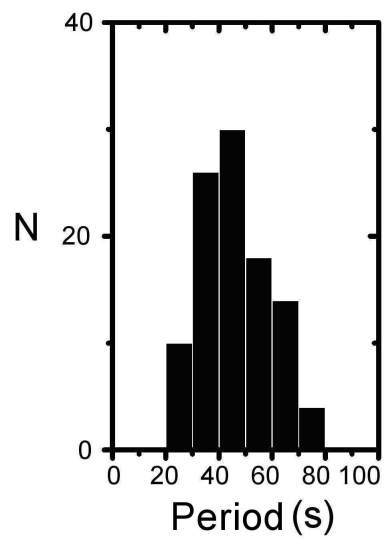

(b)

Figure 7.1. Bead size and oscillation period distributions (figure from Ref. [14]). (a) Size distribution of DOWEX 50WX4-200 (200 mesh) beads. (b) Oscillatory period distribution. Concentrations are $[\mathrm{NaBr}]=0.07 \mathrm{M},[\mathrm{MA}]=0.14 \mathrm{M},\left[\mathrm{H}_{2} \mathrm{SO}_{4}\right]=0.67 \mathrm{M}$, and $\left[\mathrm{NaBrO}_{3}\right]=$ $0.49 \mathrm{M}$.

and Fig. 7.1(b) shows the period distribution of these beads.

\subsubsection{Experimental Setup}

The reactor is a closed cylindrical vessel of diameter $1.8 \mathrm{~cm}$ with a flat viewing window on the side, as shown in Figs. 7.2(b) and 7.2(c). A combination redox electrode (MI-800 from Microelectrodes, Inc.) was used to record the electrochemical potential of the solution. The flat viewing window was illuminated by two fiber-optic lamps and the color change of the ferroin-loaded beads was photographically recorded using a digital camera (SPOT Insight IN1120) with a shutter speed of $0.4 \mathrm{~ms}$. Stirring was achieved using a magnetic stirrer (IKAMAG) and a bar of length $1.7 \mathrm{~cm}$. The stirring rate is varied between 100 and 700 rpm, in which the beads were completely suspended in the solution. 


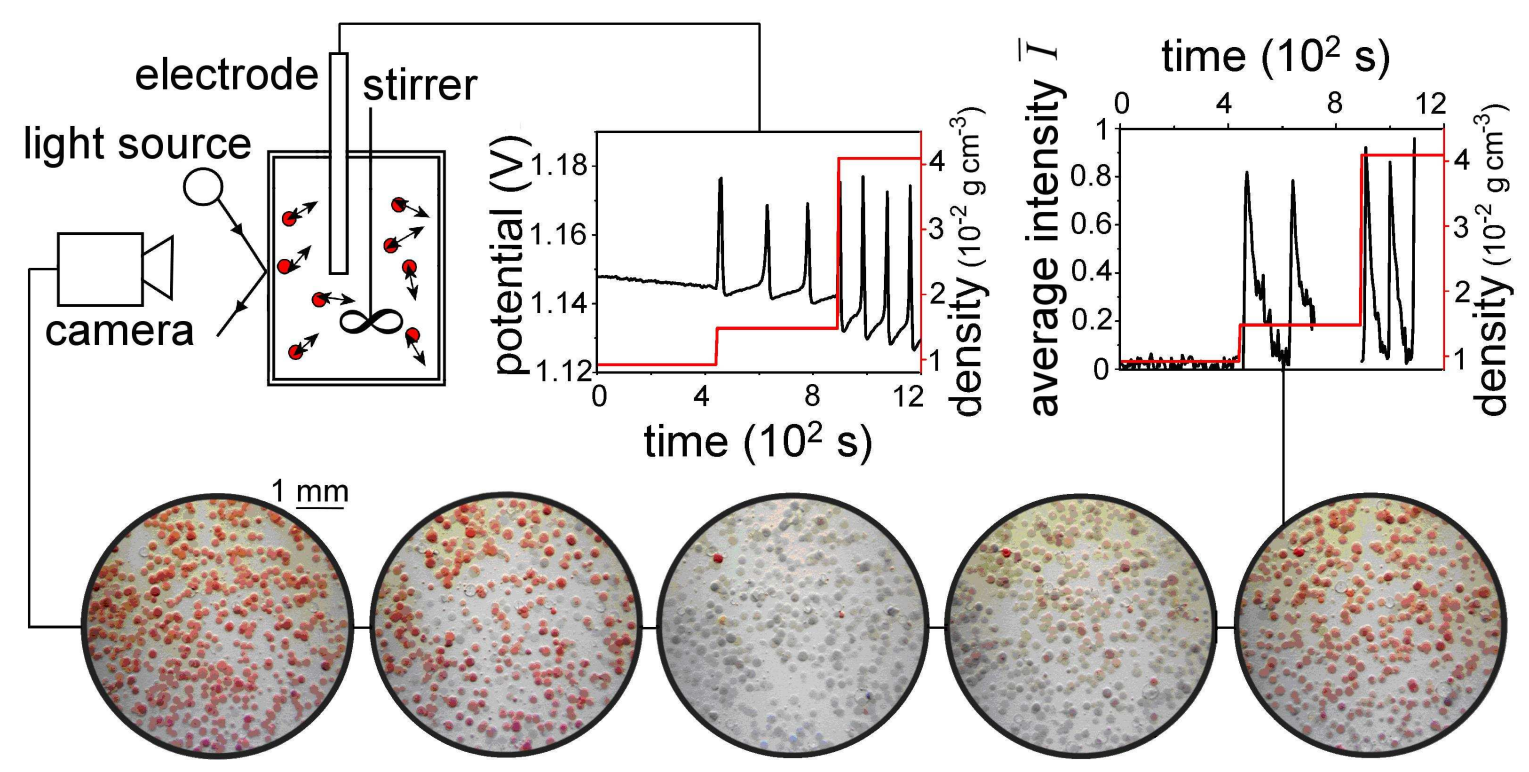

(a)

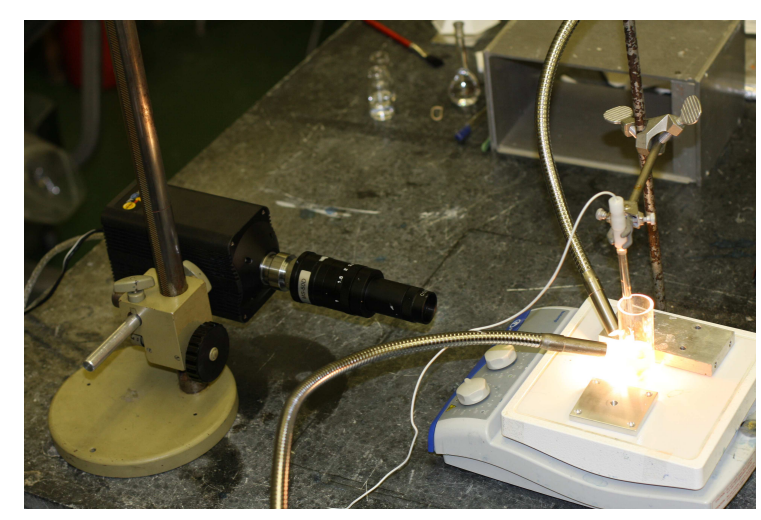

(b)

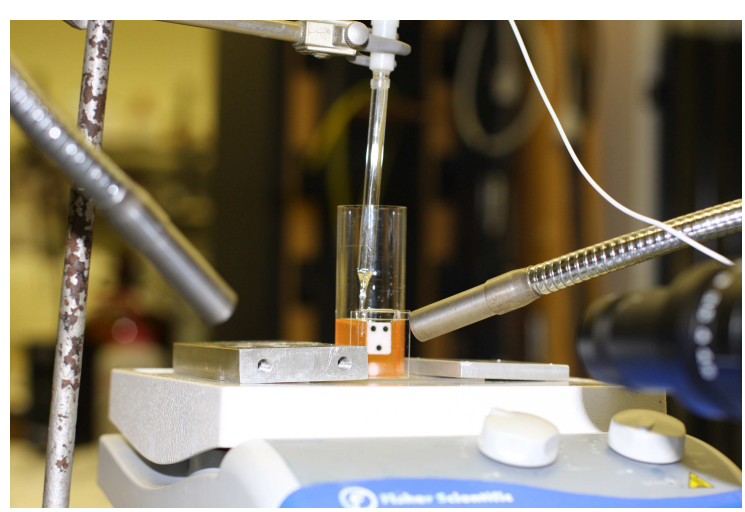

(c)

Figure 7.2. Experimental setup of the stirred system. Catalytic microparticles are globally coupled by exchange of species with the surrounding catalyst-free BZ reaction solution. (a) Sketch of the experimental setup (figure from Ref. [14]). Electrochemical time series illustrates the change in oscillatory amplitude and period with increasing particle density (red line) for a stirring rate of $600 \mathrm{rpm}$. A typical series of images obtained during one oscillation is shown from which the (normalized) average intensity of the beads is calculated as a function of time. The associated time series illustrates the change in oscillatory amplitude and period with increasing particle density (red line) for a stirring rate of $600 \mathrm{rpm}$. A density of 0.02 $\mathrm{g} \mathrm{cm}^{-3}$ corresponds to approximately $1.3 \times 10^{4}$ beads $\mathrm{cm}^{-3}$. (b) Photograph of experimental setup [14], and (c) close-up of the imaging window [14]. 


\subsubsection{Stirring Rate and Chemical Exchange Rate}

The transfer rate of chemical species between the beads and the solution can be considered as a reversible surface process [15]. The transfer rate $(v)$ can be expressed as a function of the stirring rate and is directly proportional to the concentration difference between the bead and the solution, as given by Eq. 7.2.

$$
v=-k_{e x}\left(X_{i}-X_{s}\right)
$$

where $X_{i}$ and $X_{s}$ are the concentrations of species on the bead and in the solution, respectively. $k_{e x}$ is the exchange rate constant, which is a function of the surface area $(A)$ and volume $(V)$ of the bead, as given by Eq. 7.3.

$$
k_{e x}=-k_{s l} \frac{A}{V}
$$

where $k_{s l}$ is the solid to liquid mass transfer coefficient [16]. Without stirring, $k_{s l}$ is related to the diffusion coefficient. With stirring, $k_{s l}$ is a complex function of the stirring method and the stirring rate. Experimentally determined values of $k_{s l}$ are generally in the range of $10^{-3}$ and $10^{-1} \mathrm{~cm} \mathrm{~s}^{-1}$. Studies indicate that $k_{s l}$ increases monotonically with increasing stirring rate [16]. In this work we varied the mass transfer rate by varying the stirring rate.

\subsubsection{Image Analysis}

The catalyst on the beads periodically changes its oxidation state between +2 (ferroin) and +3 (ferroin) during an oscillation, with a corresponding change in color between red and blue. In order to count the fraction of beads that lie in either of the states, the beads are first automatically identified using a code produced in-house. Then the greyscale values were added together for all beads detected (the conversion from the RGB values to greyscale values is described in Chapter 6). Finally, the fraction of the oxidized beads was calculated according to Eq. 7.4:

$$
F_{i}=\frac{\left(S(G)_{i}-S(G)_{r e d}\right) / n_{i}}{\left(S(G)_{o x}-S(G)_{r e d}\right) / N}
$$

where $F_{i}$ is the fraction of the oxidized beads for image $i, S(G)_{i}$ is the sum of the greyscale values for all of the beads in image $i$, and $S(G)_{r e d}$ and $S(G)_{o x}$ are the sum of all the 


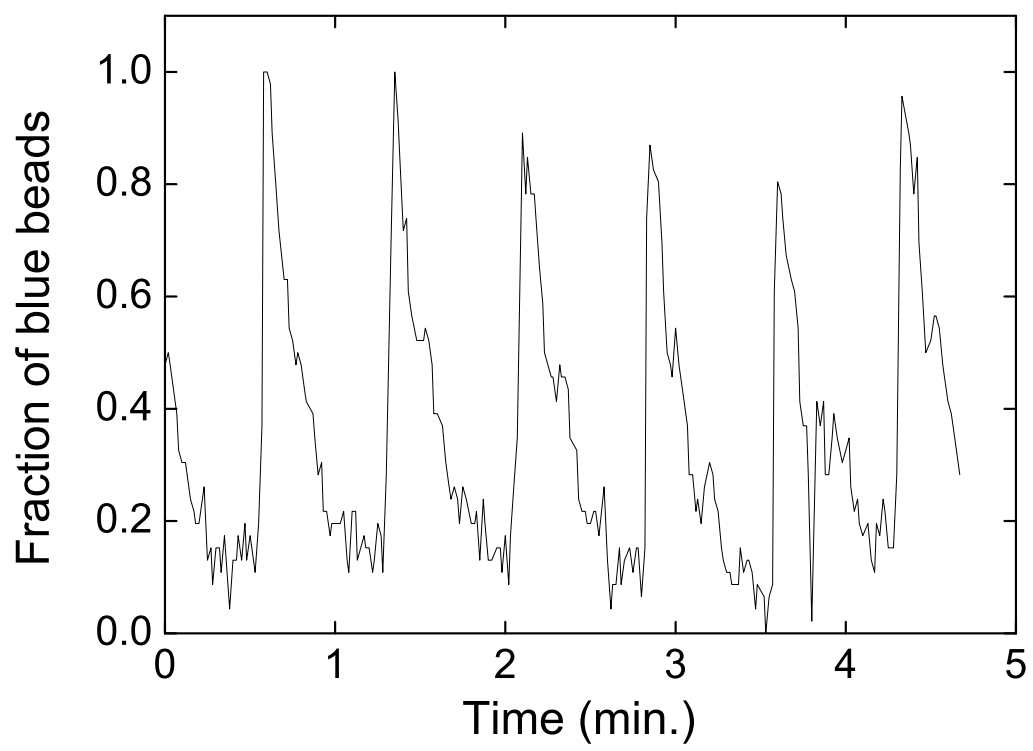

Figure 7.3. A typical plot of the fraction of oscillatory beads in the oxidized state determined with image analysis [14]. Stirring rate is $600 \mathrm{rpm}$, number density of the beads is $0.05 \mathrm{~g} \mathrm{~cm}^{-1}$, $[\mathrm{NaBr}]=0.07 \mathrm{M},[\mathrm{MA}]=0.14 \mathrm{M},\left[\mathrm{H}_{2} \mathrm{SO}_{4}\right]=0.67 \mathrm{M}$, and $\left[\mathrm{NaBrO}_{3}\right]=0.49 \mathrm{M}$.

greyscale values of the beads when the beads in the image are completely reduced or oxidized, respectively. $N$ is the number of beads in a typical image with all the beads in oxidized or reduced state and $n_{i}$ is number of beads in image $i$. The same procedure was applied to successive images, providing the time evolution of the fraction of oxidized beads.

A typical plot for an oscillation with the above image analysis is shown in Fig. 7.3.

\subsection{Experimental Results}

Two distinct types of transitions were observed depending on the stirring rate and the number density of the beads. The major characteristics of the two transitions are listed in Table 7.1.

\subsubsection{Quorum Sensing Transition}

One type of transition, which occurred at the high stirring rate of $600 \mathrm{rpm}$, was a qualitative change from steady state to oscillatory behavior, as shown in Fig. 7.4. At a low 
Table 7.1. Quorum sensing transition and Kuramoto synchronization transition

\begin{tabular}{|c|c|c|c|c|}
\hline $\begin{array}{c}\text { Stirring rate } \\
(\mathrm{rpm})\end{array}$ & Density & $\begin{array}{c}\text { Oscillatory electro- } \\
\text { chemical signal }\end{array}$ & $\begin{array}{c}\text { Same oxidation state } \\
\text { of beads in image }\end{array}$ & $\begin{array}{c}\text { Transition Type } \\
\text { (low to high density) }\end{array}$ \\
\hline \multirow{2}{*}{600} & low & No & Yes & Quorum sensing \\
\cline { 2 - 5 } & high & Yes & Yes & transition \\
\hline \multirow{2}{*}{300} & low & No & No & Kuramoto \\
\cline { 2 - 5 } & high & Yes & Yes & \\
\hline
\end{tabular}

density of beads, the global signal from the electrical measurements was nonoscillatory, and simultaneous image monitoring did not detect any oxidized beads, as shown in Figs. 7.4(a) and 7.4(d). A sudden emergence of large amplitude oscillations appeared after the number density of beads reached a critical value. An analysis of the associated images indicated that all the beads oscillated at the same frequency as the global electrical signal. A further increase of the density led to a continuous increase of the global electrical signal, as shown in Fig. 7.4(c). Further analysis of the associated images showed that all of the beads were synchronized at the same frequency and same phase. The increase of the density after the critical point caused a roughly linear increase of the global signal. The frequency of oscillations decreased when the density of beads increased. This density dependent behavior is analogous to the quorum sensing phenomenon, which occurs in certain bacteria species. We term this transition a quorum sensing transition.

\subsubsection{Kuramoto Transition}

Another type of transition occurred at a low stirring rate of $300 \mathrm{rpm}$, as shown in Fig. 7.5. At a low density of beads, a noisy global electrical signal was observed but no global oscillations existed in the solution, as shown in Fig. 7.5(a). Analysis of the simultaneously acquired images indicated that a roughly constant fraction of oxidized beads was always observed (ca. 20\%), as seen in Fig. 7.5(d). This implied that the beads were oscillating out of phase and possibly with different frequencies. Increases in the bead density led to a 


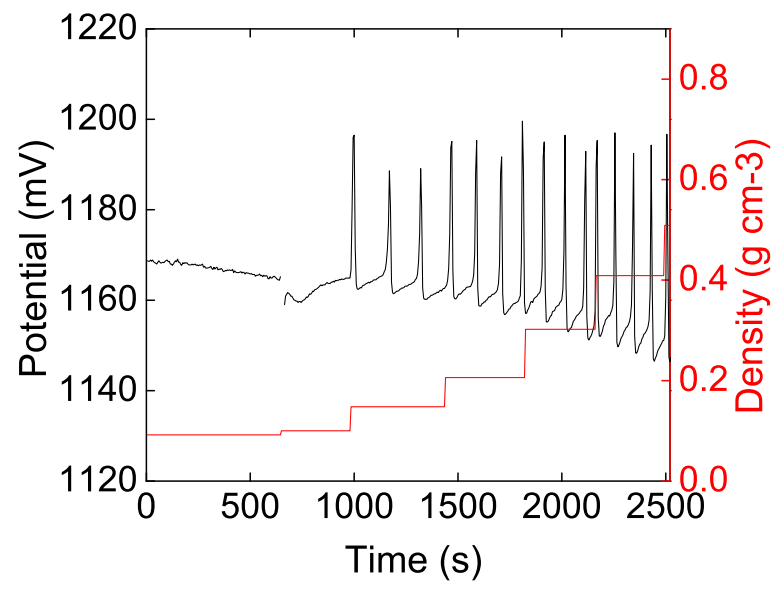

(a)

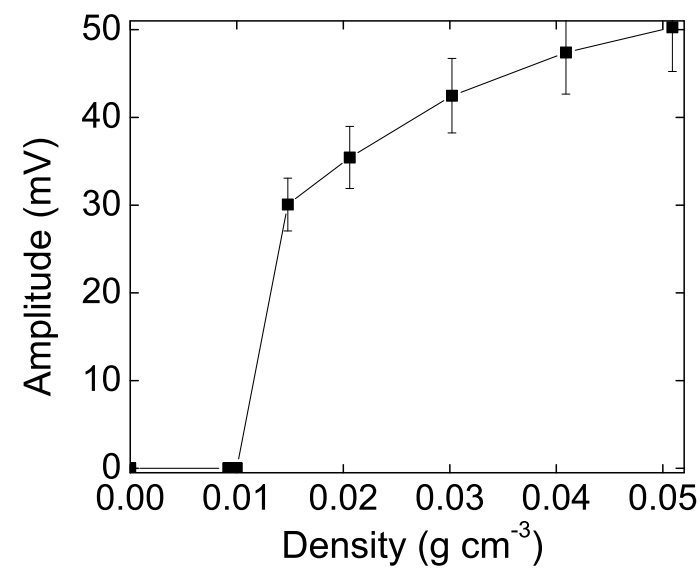

(c)

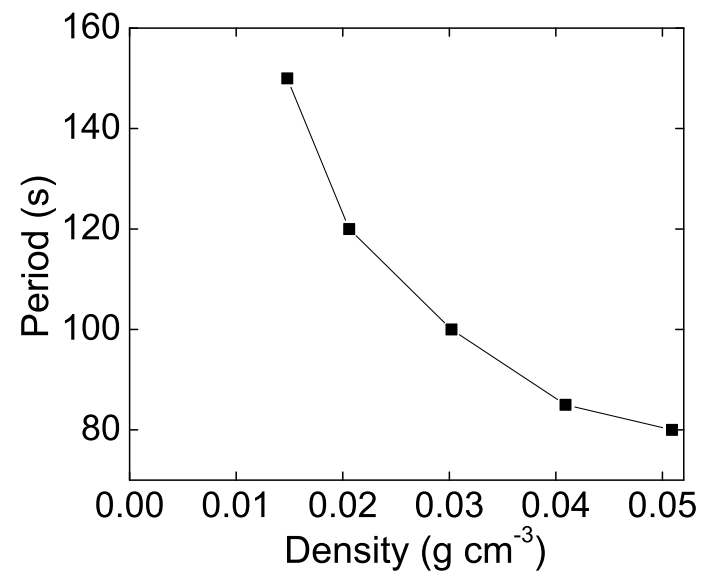

(b)

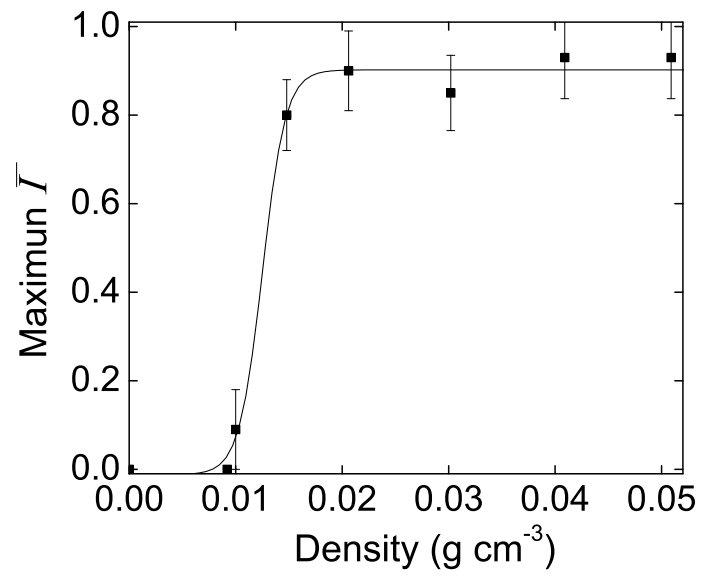

(d)

Figure 7.4. Quorum sensing transition in the stirred system. Stirring rate is $600 \mathrm{rpm}$, [NaBr] $=0.07 \mathrm{M},[\mathrm{MA}]=0.14 \mathrm{M},\left[\mathrm{H}_{2} \mathrm{SO}_{4}\right]=0.67 \mathrm{M}$, and $\left[\mathrm{NaBrO}_{3}\right]=0.49 \mathrm{M}$. (a) Typical time series for the solution potential [14], (b) the dependence of the oscillation period (figure from Ref. [14]), (c) the dependence of the oscillation amplitude (figure from Ref. [14]), and (d) the dependence of the normalized intensity on the number density of the beads (figure from Ref. [14]). 
gradual increase of both the global oscillation amplitudes and the fraction of the oxidized beads, as shown in Figs. $7.5(\mathrm{c})$ and $7.5(\mathrm{~d})$. There was no sudden change in either signal. The oscillation period decreased from $42 \mathrm{~s}$ to $32 \mathrm{~s}$ when the density was increased from 0.01 to $0.04 \mathrm{~g} \mathrm{~cm}^{-3}$, compared to the quorum sensing transitions, which decreased from $150 \mathrm{~s}$ to $85 \mathrm{~s}$ for a density change from 0.01 to $0.04 \mathrm{~g} \mathrm{~cm}^{-3}$, as seen in Fig. 7.5(b).

\subsection{Model Studies}

\subsubsection{Simulation Methods}

Numerical simulations were carried out with the ZBKE particle model [17] described in Chapter 6. Each bead is treated as a minireactor with exchange of activator $\left(\mathrm{HBrO}_{2}\right)$ and inhibitor $\left(\mathrm{Br}^{-}\right)$with the solution. The concentrations of the activator of bead $i$ and the solution are described by Eq. 7.5 and 7.6, respectively:

$$
\begin{gathered}
\frac{d X_{i}}{d t}=-k_{e x}\left(X_{i}-X_{s}\right)+f\left(X_{i}, Y_{i}, Z_{i}\right) \\
\frac{d X_{s}}{d t}=\frac{\bar{V}}{V_{s}} \sum i^{N} k_{e} x\left(X_{i}-X_{s}\right)+g\left(X_{s}, Y_{s}\right)
\end{gathered}
$$

where $X_{s}$ and $X_{i}$ are the concentrations of the activator in the solution and on bead $i$, $f\left(X_{i}, Y_{i}, Z_{i}\right)$ represents the chemical reaction on the beads, and $g\left(X_{s}, Y_{s}\right)$ represents the chemical reaction in the solution. Functions $f\left(X_{i}, Y_{i}, Z_{i}\right)$ and $g\left(X_{s}, Y_{s}\right)$ incorporate the ZBKE model chemistry [17]. The term $k_{e x}\left(X_{i}-X_{s}\right)$ represents the exchange rate of the activator between bead $i$ and the solution, and $k_{e x}$ is the exchange rate constant, which increases with increasing stirring rate [16], as introduced in Section 7.2.3. The dilution

factor is $\frac{\bar{V}}{V_{s}}$, where $\bar{V}$ and $V_{s}$ represent the average volume of the bead and volume of the solution, respectively.

The concentration of the inhibitor on the bead and in the solution is described by expressions analogous to Eq. 7.5 and 7.6. The concentration of catalyst on the beads is determined by the loading and there is no exchange between the beads and the solution. The explicit 


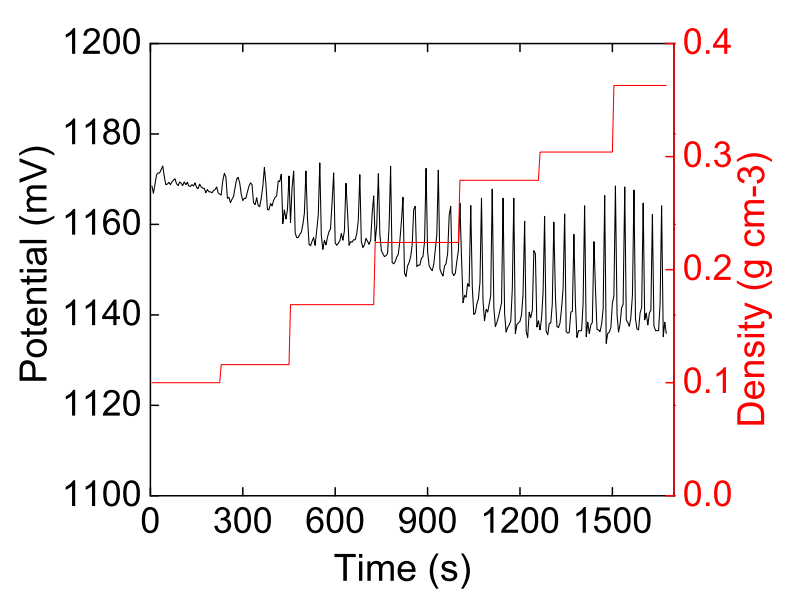

(a)

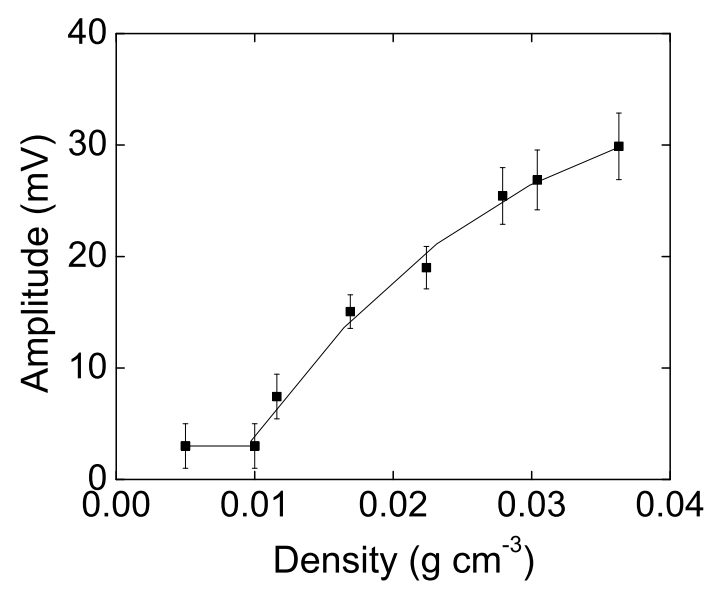

(c)

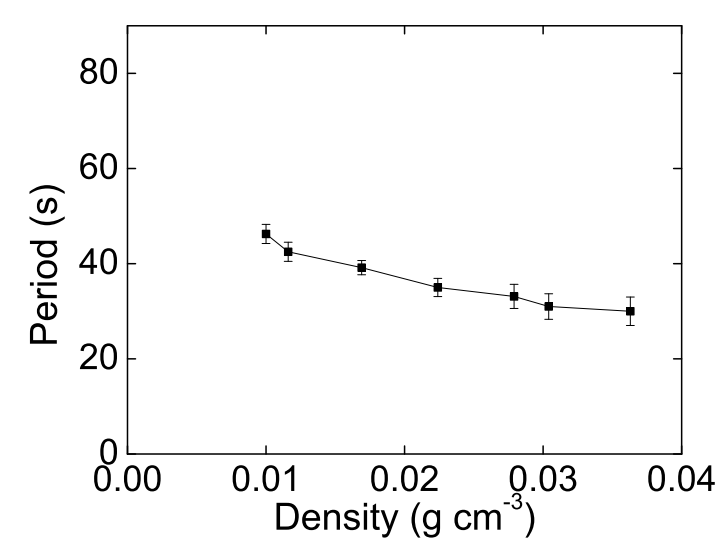

(b)

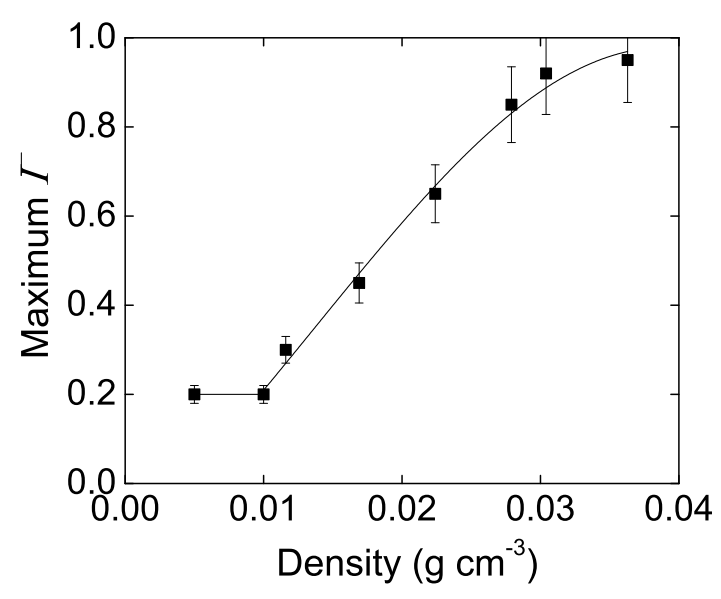

(d)

Figure 7.5. Kuramoto type synchronization transition in the stirred system. Stirring rate is $300 \mathrm{rpm},[\mathrm{NaBr}]=0.07 \mathrm{M},[\mathrm{MA}]=0.14 \mathrm{M},\left[\mathrm{H}_{2} \mathrm{SO}_{4}\right]=0.67 \mathrm{M}$, and $\left[\mathrm{NaBrO}_{3}\right]=0.49 \mathrm{M}$. (a) A typical time series for the solution potential [14], (b) the dependence of the oscillation period (figure from Ref. [14]), (c) the dependence of the oscillation amplitude (figure from Ref. [14]), and (d) the dependence of the normalized intensity on the number density of the beads (figure from Ref. [14]) 
evolution of the activator $X$ and the inhibitor $Y$ is given by:

$$
\begin{gathered}
\frac{d X_{i}}{d t}=-k_{e x}\left(X_{i}-X_{s}\right)-k_{2} h_{0} X_{i} Y_{i}+k_{3} h_{0} A Y_{i}-2 k_{4} X_{i}^{2}-k_{5} h_{0} A X_{i} \\
+k_{-5} U_{s s}^{2}+k_{6} U_{s s}\left(C-Z_{i}\right)-k_{-6} X_{i} Z_{i} \\
\frac{d Y_{i}}{d t}=-k_{e x}\left(Y_{i}-Y_{s}\right)-k_{2} h_{0} X_{i} Y_{i}-k_{3} h_{0} A Y_{i}+q_{i} \frac{k_{7} k_{8} B Z_{i}}{k_{-7} h_{0}\left(C-Z_{i}\right)+k_{8}}+k_{9} B \\
\frac{d X_{s}}{d t}=\frac{\bar{V}}{V_{s}} \sum i^{N} k_{e} x\left(X_{i}-X_{s}\right)-k_{2} h_{0} X_{i} Y_{i}+k_{3} h_{0} A Y_{i}-2 k_{4} X_{i}^{2}-k_{5} h_{0} A X_{i}+k_{-5} U_{s s}^{2} \\
\frac{d Y_{s}}{d t}=\frac{\bar{V}}{V_{s}} \sum i^{N}-k_{2} h_{0} X_{s} Y_{s}-k_{3} h_{0} A Y_{s}+k_{9} B
\end{gathered}
$$

The concentration of the oxidized catalyst on the bead is given by:

$$
\frac{d Z_{i}}{d t}=k_{6} U_{s s}\left(C-Z_{i}\right)-k_{-6} X_{i} Z_{i}-\frac{k_{7} k_{8} B Z_{i}}{k_{-7} h_{0}\left(C-Z_{i}\right)+k_{8}}
$$

where $U_{s s}$ is steady state concentration of $\left[\mathrm{HBrO}_{2}^{+}\right]$, which is given by Eq. 7.12.

$$
U_{s s}=\frac{1}{4 k_{-5}}\left(-k_{6}\left(C-Z_{i}\right)+\left(k_{6}^{2}\left(C-Z_{i}^{2}\right)+16 k_{-5} k_{5} h_{0} A x_{i}+8 k_{-5} k_{-6} x_{i} z_{i}\right)^{1 / 2}\right)
$$

The system of equations is integrated using the Euler method with $d t=0.001 \mathrm{~s}$.

The synchronization of the beads is characterized by an order parameter $K$, which was introduced by Shinomoto and Kuramoto [19]. The determination of $K$ was carried out for $N=1000$ beads with Eq. 7.13.

$$
K=<\left|N^{-1} \sum e^{i \theta_{j}}-<N^{-1} \sum e^{i \theta_{j}}>\right|>
$$

where $\theta_{j}$ is the phase of oscillator $j$ and $<>$ represents the average over time. When the oscillators are out of phase or they do not oscillate, $K=0$. When the oscillators are in perfect synchrony, $K=1$. Table 7.2 lists all the parameters used in the simulations. 


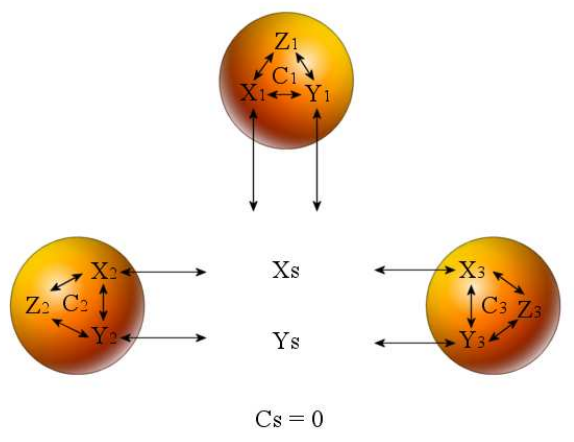

Figure 7.6. Numerical modeling setup (figure adapted from Ref. [18]). Each bead is a minireactor with exchange of activator $(X)$ and inhibitor $(Y)$ with the surrounding solution. There is no catalyst $(Z)$ in solution. The concentration of catalyst in each bead is different, which contributes to different oscillation period of each bead.

Table 7.2. The value of parameters in the ZBKE model

\begin{tabular}{|c|c|c|c|c|c|}
\hline Parameter & Value & Parameter & Value & Parameter & Value \\
\hline $\mathrm{A}(\mathrm{M})$ & 0.39 & $k_{2}\left(M^{-2} s^{-1}\right)$ & $7.6 \times 10^{6}$ & $k_{-6}\left(M^{-1} s^{-1}\right)$ & 0.30 \\
\hline $\mathrm{B}(\mathrm{M})$ & 0.14 & $k_{3}\left(M^{-2} s^{-1}\right)$ & 2.0 & $k_{8} / k_{-7}\left(M^{2}\right)$ & $3.0 \times 10^{-6}$ \\
\hline $\mathrm{C}(\mathrm{M})$ & $1.7 \times 10^{-3}$ & $k_{4}\left(M^{-1} s^{-1}\right)$ & 8600 & $k_{8} k_{7} / k_{-7}\left(M s^{-1}\right)$ & $5.0 \times 10^{-7}$ \\
\hline$h_{0}$ & 0.77 & $k_{5}\left(M^{-2} s^{-1}\right)$ & 10.0 & $k_{9}\left(s^{-1}\right)$ & $3.3 \times 10^{-6}$ \\
\hline $\bar{q}$ & 0.6 & $k_{-5}\left(M^{-1} s^{-1}\right)$ & $4.2 \times 10^{6}$ & $k_{e x}\left(s^{-1}\right)$ & $0.30-30$ \\
\hline$\sigma_{q}$ & 0.05 & $k_{6}\left(M^{-1} s^{-1}\right)$ & $1.66 \times 10^{7}$ & $\bar{V} / V_{s}$ & $1.0 \times 10^{-6}$ \\
\hline
\end{tabular}




\subsubsection{Quorum Sensing and Kuramoto Transitions}

The various transitions with changing $k_{e x}$ and the density $n$ become apparent in a surface plot of the amplitude or the order parameter $K$. Figure 7.7 shows plots of $\left[\mathrm{HBrO}_{2}\right]$ amplitude and $K$ as a function of exchange rate $k_{e x}$ and number density $n$. The quorum sensing transition occurs at high $k_{e x}$, as indicated by the green line in Fig. 7.7(a). At low number density of the beads and high $k_{e x}$, the concentration of bromous acid is at its base level. The beads are nonoscillatory and in a stationary state. With increasing number density, there is a sharp change for both the maximum concentration of bromous acid and the coherence factor $K$. At all densities for high $k_{e x}$, there is always either a steady state or a single oscillation frequency, as shown in Fig. 7.8(a).

The Kuramoto transition occurs at lower values of $k_{e x}$, where an increase in the number density leads to a gradual increase of the maximum bromous acid concentrations and the coherence factor $K$. Most of the beads are oscillatory for any number density; however, at low densities, there is a distribution of the oscillating frequencies and phases, as shown in Fig. 7.8(b).

\subsubsection{Effect of Exchange Rate on Transitions}

Figure 7.9 shows how the exchange rate affects the transitions with constant number density of the beads. With increasing exchange rate, the oscillation amplitude increases continuously, while a further increase of the exchange rate causes a sudden disappearance of the oscillation signal, as shown in Fig. 7.9(a). The first appearance of oscillations is a Kuramoto transition, and the sudden disappearance of oscillators is a quorum sensing transition. Both the Kuramoto and quorum sensing transitions were observed experimentally, as shown in the lower panel. The concentration of activator on a single bead and in solution during an oscillation is shown in Fig. 7.9(b). This figure also shows the loss rate of activator as a function of time. The sudden disappearance of oscillations on decreasing the exchange rate is seen in the simulations, as shown in Figs. 7.9(c) and 7.7(a) (pink line). Figure 7.9(d) shows the activator concentration in solution, the average value for the beads, and the loss rate of activator from the beads to the solution during the transition from steady state to 


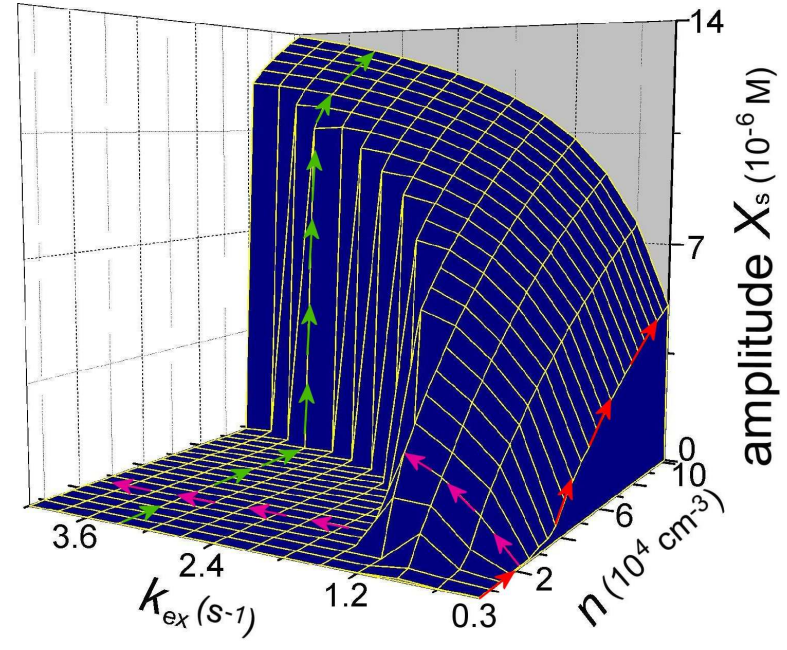

(a)

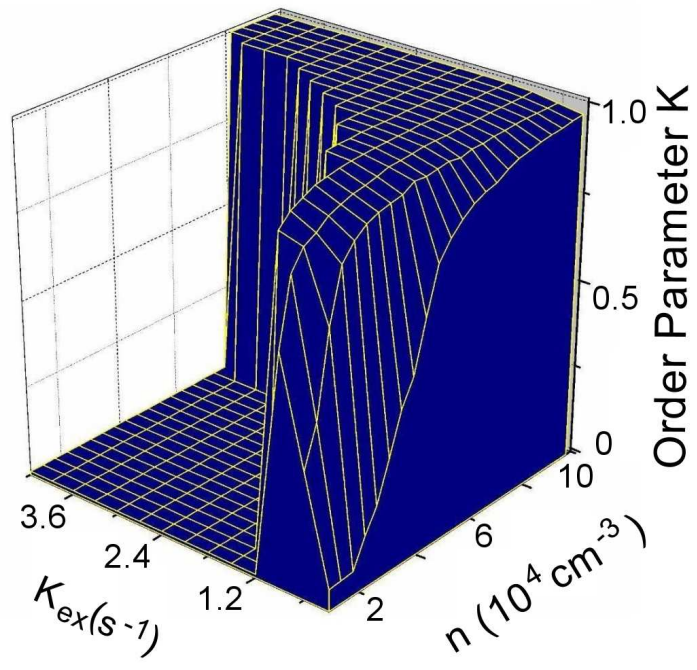

(b)

Figure 7.7. Surface plots for maximum bromous acid concentration and order parameter $K$. (a) Maximum bromous acid concentration (figure from Ref. [14]). Kuramoto synchronization at low exchange rate and quorum sensing transition at high exchange rate are indicated by red and green arrows, respectively. The pink arrows indicate a gradual increase of maximum bromous acid concentration followed by a quorum sensing transition when the exchange rate was varied at a constant number density of beads. (b) Variation of the order parameter $K$ under same conditions as in (a) [14]. 

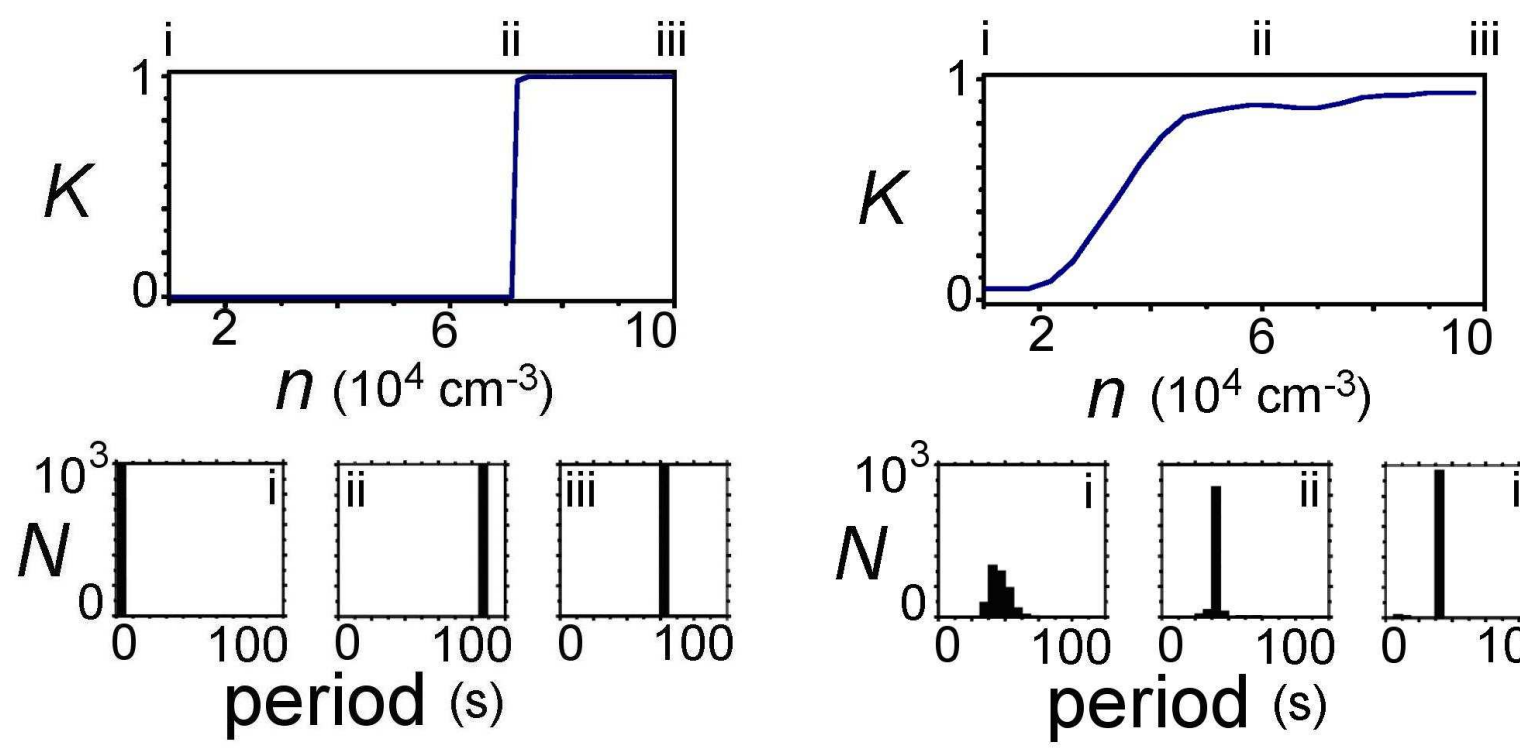

(a)

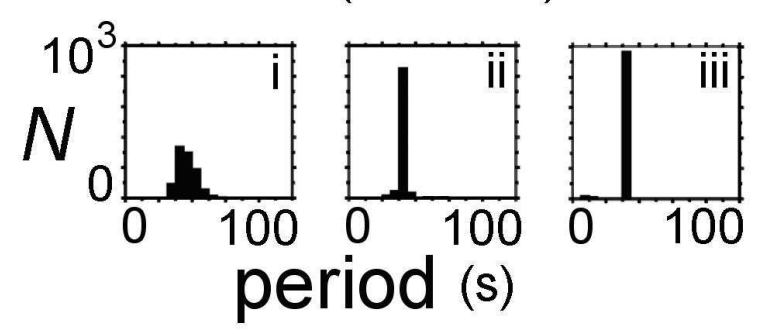

(b)

Figure 7.8. Kuramoto synchronization transition and quorum sensing transition in globally coupled particle model (figure from Ref. [14]). (a) Order parameter $K$ as a function of number density $n$ showing quorum sensing transition. Bottom panel shows the period distribution at different number densities $n=6$ (i), 8 (ii), and $10 \times 10^{4} \mathrm{~cm}^{-3}$ (iii). (b) Order parameter $K$ as a function of number density $n$ showing Kuramoto synchronization transition. Bottom panel shows the period distribution at different number densities $n=2$ (i), 6 (ii), and $10 \times 10^{4} \mathrm{~cm}^{-3}$ (iii). 
oscillatory behavior.

\subsection{Comparison between Locally and Globally Cou- pled Systems}

Our experimental system gives us an opportunity to explore different types of collective behavior that can occur under various conditions of coupling and density. Insights into these emergent behaviors can be gained by conducting modeling studies of the various systems. The well-stirred system is modeled using the three-variable ZBKE model [17], with the variables $X_{i}, Y_{i}$ and $Z_{i}$ representing $\left[\mathrm{HBrO}_{2}\right],\left[\mathrm{Br}^{-}\right]$, and the oxidized form of the catalyst, respectively, on particle $i$. The solution phase chemistry is modeled with the two variables $X_{s}$ and $Y_{s}$, which represent the solution phase concentrations of $\left[\mathrm{HBrO}_{2}\right]$ and $\left[\mathrm{Br}^{-}\right]$, respectively. The kinetic equations incorporate terms for the rates of reaction between the species and an additional term to account for the solution-particle exchange of bromous acid and bromide. For bromous acid, this exchange term for an individual particle is given by $k_{e x}\left(X_{i}-X_{s}\right)$, where $k_{e x}$ is the exchange rate, which depends on the stirring speed. The spatial system may be similarly modeled using a 3D grid of particle-cells and solution-cells. The particlecells are positioned in a square or rectangular arrangement on the lowest layer of the 3D grid. Diffusive coupling between neighboring cells is modeled using a six-point Laplacian. Heterogeneity in the natural frequency of the particles is included in both systems by varying the stoichiometric coefficient or the total catalyst loading for the individual particles.

The transitions in the stirred system with low $k_{e x}$ serve as examples of population size dependent synchronization phenomena. Modeling studies indicate that synchronization occurs through increases in the concentration of the autocatalytic $\mathrm{HBrO}_{2}$ in the surrounding solution. Increasing the number density of particles leads to more of them firing together with a resultant increase in the amplitude of the $\mathrm{HBrO}_{2}$ pulse in the surrounding solution. At a sufficient number density, the particles become phase and frequency synchronized.

This behavior contrasts with the spatially distributed system of oscillatory particles, in which only frequency synchronization occurs. Examination of the spatiotemporal patterns 


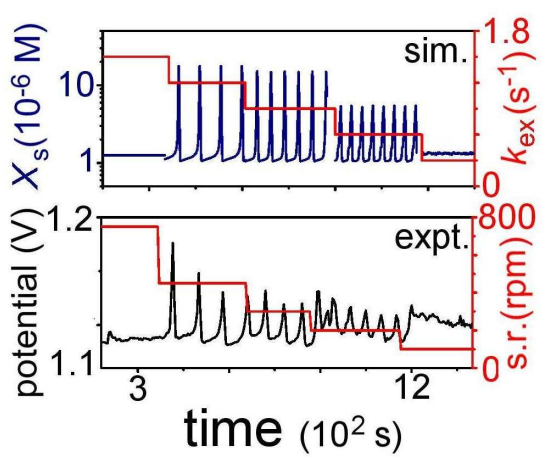

(a)

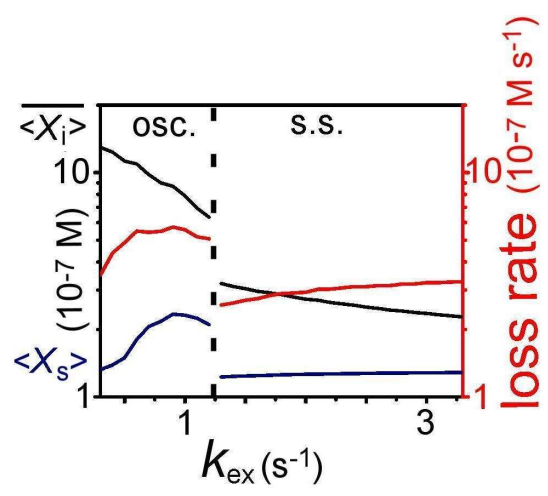

(c)

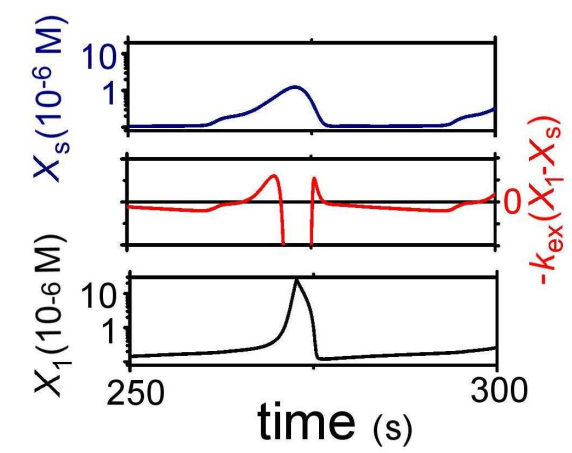

(b)

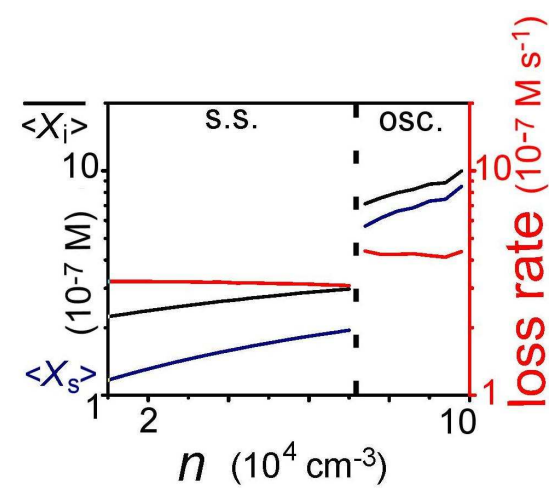

(d)

Figure 7.9. Influence of exchange rate on the transitions (figure from Ref. [14]). (a) Desynchronizations of oscillators with decreasing $k_{e x}\left(n=1.8 \times 10^{4} \mathrm{~cm}^{-3}\right)$ and stirring rate (density $=0.0162 \mathrm{~cm}^{-3}$ ). (b) Concentration of activator for a bead, in the solution, and the exchange rate $-k_{e x}\left(X_{i}-X_{s}\right)$ during one oscillation cycle. Experimental conditions are $n=1.8 \times 10^{4} \mathrm{~cm}^{-3}$ and $k_{e x}=0.3 \mathrm{~s}^{-1}$. (c) Transitions from oscillatory behavior to steady state with increasing exchange rate. Black and blue lines represent the time-averaged activator concentration for the beads and the solution, respectively. Red line represents the loss rate of activator from the bead $\left(<k_{e x}\left(X_{i}^{-}-X_{s}\right)>\right)$. (d) Transition from steady state to oscillatory behavior with increasing $n\left(k_{e x}=3.0 \mathrm{~s}^{-1}\right)$. 
in Fig. 6.8 shows that there are one or more reentrant circuits [20] situated around small holes in the medium that serve as the source. The tip of the spiral structure shown in Fig. 6.8(b) is easily identified as a reentrant circuit. Local coupling by reaction-diffusion leads to the propagation of the wave from this center across the group, frequency locking the activity of each particle [21]. The increase in complexity with increasing bromate concentration has been reported previously by a number of authors $[22,23]$. This phenomenon and the initial origin of the reentrant circuits will be the subject of further work.

The well-stirred experiment with high $k_{e x}$ and the experiment with spatially distributed excitable beads both display quorum sensing transitions. In both cases, small numbers of particles remain in a quiescent state but, on increasing the number of particles, a sharp transition to synchronized oscillations occurs. In the well stirred system, the synchronization is complete. In the spatial system, the particles are frequency synchronized and are linked by an organizing center, which can be a pacemaker particle or a reentrant center.

In many studies $[24,25]$ of quorum sensing transitions in bacterial populations, a build up of an autoinducer species in the surrounding solution is used to explain the behavior. However, a loss based explanation of quorum sensing in a model of the bacteria Pseudomonas aeruginosa has been proposed [26]. At high stirring rate and low density in the well-stirred system, $\mathrm{HBrO}_{2}$ loss from the particles is high and the particles are in a steady state. On increasing the number density, $\left[\mathrm{HBrO}_{2}\right]$ increases in the surrounding solution, which leads to a decrease in the loss rate from an individual particle. At a threshold value, the individual particles are capable of supporting oscillation.

The quorum sensing transitions serve as examples of collective phenomena. At the critical number of particles, the removal of any single member will result in all community members remaining in a steady state. For example, in the spatially distributed system, the removal of a single particle can result in the pacemaker particle remaining quiescent rather than becoming active.

The microreactors studied in this work demonstrate a diverse range of behaviors. In the spatially distributed system, a number of questions remain including the origin and evolution of the broken wave patterns. The degree of heterogeneity in the population is likely to play a key role in the formation of these patterns as well as in the development of pacemakers. 
Table 7.3. Comparison between the spatial and stirred systems

\begin{tabular}{|c|c|c|c|c|c|}
\hline System & $\begin{array}{c}\text { Coupling } \\
\text { type }\end{array}$ & $\begin{array}{c}\text { S.S. to Osc. } \\
\text { Transition }\end{array}$ & $\begin{array}{c}\text { Synchronization } \\
\text { of oscillators }\end{array}$ & {$\left[\mathrm{HBrO}_{2}\right]$} & $\begin{array}{c}\text { Increased } \\
\text { term with } \mathrm{n} \uparrow\end{array}$ \\
\hline Spatial & local & yes & $\begin{array}{c}\text { frequency } \\
\text { synchronization }\end{array}$ & $\begin{array}{c}{\left[\mathrm{HBrO}_{2}\right] \downarrow} \\
\text { with } \mathrm{n} \uparrow\end{array}$ & $\begin{array}{c}\left(\mathrm{HBrO}_{2} \mathrm{Br}^{-}\right)_{\text {loss }} \\
\text { and }\left[\mathrm{Br}^{-}\right]_{\mathrm{s}}\end{array}$ \\
\hline Stirred & global & yes & $\begin{array}{c}\text { frequency and phase } \\
\text { synchronization }\end{array}$ & $\begin{array}{c}{\left[\mathrm{HBrO}_{2}\right] \uparrow} \\
\text { with } \mathrm{n} \uparrow\end{array}$ & $\begin{array}{c}{\left[\mathrm{HBrO}_{2}\right]_{\mathrm{s}},\left[\mathrm{Br}^{-}\right]_{\mathrm{s}}} \\
\text { and }\left(\mathrm{HBrO}_{2}\right)_{\text {loss }}\end{array}$ \\
\hline
\end{tabular}

Heterogeneity also plays a role in the behavior of the well-stirred system.

\subsection{Conclusion}

Two different types of transitions, a quorum sensing transition and a Kuramoto synchronization transition, were observed with increasing bead density in a globally coupled oscillator system. The coupling between the oscillators was achieved through the exchange of the activator $\mathrm{HBrO}_{2}$ via the solution. At low stirring rates, the exchange rate is low and the low coupling strength led to a gradual synchronization of the oscillators. This transition is an example of a Kuramoto transition. At high stirring rates, the exchange rate of the activator is high and the high coupling strength led to a sharp transition from steady state to perfectly synchronized oscillatory behavior. This is an example of a dynamical quorum sensing transition.

Numerical studies carried out with the three-variable ZBKE model [17] reproduced both transitions at the corresponding exchange rates. The simulations also predicted two transitions for heterogeneous beads when the exchange rate was varied and the number density was held constant. The prediction was in accord with the experimental observations. 


\section{Bibliography}

[1] A. T. Winfree, The Geometry of Biological Time (Springer, New York, USA, 2001), 2nd edition. 122

[2] A. Goldbeter, Biochemical Oscillations and Cellular Rhythms: The Molecular Bases of Periodic and Chaotic Behavior (Cambridge Univ. Press, Cambridge, UK, 1996). 122

[3] L. Glass and M. C. Mackey, From Clocks to Chaos: The Rhythms of Life (Princeton Univ. Press, Princeton, NJ, USA, 1988). 122

[4] J. Cui, Synchronization of Spatiotemporal Patterns and Modeling Disease Spreading Using Excitable Media, Ph.D. thesis, West Virginia University (2004). 122

[5] Y. Kuramoto, Chemical Oscillations, Waves and Turbulence (Springer, Berlin, Germany, 1984). 122

[6] A. T. Winfree, "On emerging coherence," Science 298, 2336-2337 (2002).

Online Version 122

[7] V. K. Vanag and I. R. Epstein, "Inwardly rotating spiral waves in a reaction-diffusion system," Science 294, 835-837 (2001). 122

[8] J. Garcia-Ojalvo, M. B. Elowitz, and S. H. Strogatz, "Modeling a synthetic multicellular clock: Repressilators coupled by quorum sensing," Proc. Nat. Acad. Sci. 101, 1095510960 (2004).

Online Version 122 
[9] G. Gerisch and B. Hess, "Cyclic-AMP-controlled oscillations in suspended Dictyostelium cells: Their relation to morphogenetic cell interactions," Proc. Nat. Acad. Sci. 71, 21182122 (1974).

Online Version 122

[10] I. Z. Kiss, Y. Zhai, and J. L. Hudson, "Emerging coherence in a population of chemical oscillators," Science 296, 1676-1678 (2002).

Online Version 122

[11] J. Aldridge and E. K. Pye, "Cell density dependence of oscillatory metabolism," Nature 259, 670-671 (1976).

Online Version 123

[12] S. Danø, P. G. Sørensen, and F. Hynne, "Sustained oscillations in living cells," Nature 402, 320-322 (1999).

Online Version 123

[13] S. D. Monte, F. d'Ovidio, S. Danø, and P. G. Sørensen, "Dynamical quorum sensing: Population density encoded in cellular dynamics," Proc. Natl. Acad. Sci. USA 104, 18377-18381 (2007).

Online Version 123

[14] A. F. Taylor, M. Tinsley, F. Wang, Z. Huang, and K. Showalter, "Dynamical quorum sensing and synchronization in large populations of chemical oscillators," Science 323, 614-617 (2009). 124, 125, 127, 129, 131, 135, 136, 138

[15] L. D. Schmidt, The Engineering of Chemical Reactions (Oxford Univeristy Press, 1998). 126

[16] V. G. Pangarkar, A. A. Yawalkar, M. M. Sharma, and A. A. C. M. Beenackers, "Particleliquid mass transfer coefficient in two-/three-phase stirred tank reactors," Ind. Eng. Chem. Res. 41, 4141-4167 (2002).

Online Version 126, 130 
[17] A. M. Zhabotinsky, F. Buchholtz, A. B. Kiyatkin, and I. R. Epstein, "Oscillations and waves in metal-ion-catalyzed bromate oscillating reactions in highly oxidized states," J. Phys. Chem. 97, 1518-7584 (1993).

Online Version 110, 130, 137, 140

[18] R. Toth, A. F. Taylor, and M. R. Tinsley, "Collective behavior of a population of chemically coupled oscillators," J. Phys. Chem. B 110, 10170-10176 (2006).

Online Version 98, 100, 102, 133

[19] S. Shinomoto and Y. Kuramoto, "Phase transitions in active rotator systems," Prog. Theo. Phys. 75, 1105-1110 (1986).

Online Version 132

[20] J. E. Strang and P. Osborn, "Wave patterns in frequency-entrained oscillator lattices," Phys. Rev. E 72, 056137 (2005).

Online Version 110, 139

[21] O.-U. Kheowan, E. Mihaliuk, B. Blasius, I. Sendiña-Nadal, and K. Showalter, "Wave mediated synchronization of nonuniform oscillatory media," Phys. Rev. Lett. 98, 074101 (2007).

Online Version 139

[22] J. Maselko, J. S. Reckley, and K. Showalter, "Regular and irregular spatial patterns in an immobilized-catalyst Belousov-Zhabotinsky reaction," J. Phys. Chem. 93, 27742780 (1989).

Online Version 139

[23] R. Toth and A. F. Taylor, "Loss of coherence in a population of diffusively coupled oscillators," J. Chem. Phys. 125, 224708 (2006).

Online Version 139

[24] E. P. Greenberg, "Quorum sensing in gram-negative bacteria," ASM News 63, 371-377 (1997).

Online Version 139 
[25] A. Camilli and B. L. Bassler, "Bacterial small-molecule signalling pathways," Science 311, 1113-1116 (2006).

Online Version 139

[26] J. D. Dockery and J. P. Keener, "A mathematical model for quorum sensing in Pseudomonas aeruginosa," Bull. Math. Biol. 63, 95-116 (2001).

Online Version 139 


\section{Appendix A}

\section{Abbreviations for Monomers and Crosslinkers}

Most monomers and crosslinkers have long names and abbreviations are commonly used in order to simplify the notation. Tables A.1 and A.2 list the abbreviations for the monomers and crosslinkers described in this dissertation. 
Table A.1. Abbreviations in dissertation (Part 1)

\begin{tabular}{|c|c|c|}
\hline Type & Full name & Abbreviation \\
\hline \multirow{4}{*}{ Monomer } & $N$-isopropylacrylamide & NIPAAm \\
\cline { 2 - 3 } & $N, N$-diethylacrylamide & DEAAm \\
\cline { 2 - 3 } & $N$-acryloyl- $N^{\prime}$-methyl piperazine & AcrNMP \\
\cline { 2 - 3 } & $N$-acryloyl- $N^{\prime}$-ethyl piperazine & AcrNEP \\
\cline { 2 - 3 } & Acrylamide & AAm \\
\cline { 2 - 3 } & 2 -hydroxypropyl methacrylate & HPMA \\
\cline { 2 - 3 } & $N, N^{\prime}$-dimethylaminoethyl methacrylate & DMAEMA \\
\cline { 2 - 3 } & $N$-acryloyl- $N^{\prime}$-alkylpiperazine & NANAP \\
\cline { 2 - 3 } & $n$-alky methacrylate easter & AMAE \\
\cline { 2 - 3 } & ethylene glycol & EG \\
\cline { 2 - 3 } & Acrylic Acid & AAc \\
\hline
\end{tabular}


Table A.2. Abbreviations in dissertation (Part 2)

\begin{tabular}{|c|c|c|}
\hline Type & Full name & Abbreviation \\
\hline \multirow{11}{*}{ Monomer } & methacrylic acid & MAAc \\
\hline & 2-hydroxyethyl methacrylate & HEMA \\
\hline & ethylenglycol dimethylacrylate & EGDMA \\
\hline & sodium methacrylate & SMA \\
\hline & vinylmethylether & VMEG \\
\hline & butylmethacrylate & BMA \\
\hline & $N$-acryloyl pyrrolidine & APy \\
\hline & 2-carboxyisopropylacrylamide & CIPAAm \\
\hline & trimethyl( $N$-acryloyl-3-aminopropyl)ammonium iodide & TMAAPAI \\
\hline & 2-methyl-2-acrylamido-propanesulfonic acid & AMPS \\
\hline & Sulfadimethoxine monomer & SDM \\
\hline \multirow{3}{*}{ Crosslinker } & N,N'-Methylenebisacrylamide & MBAA \\
\hline & Ethylenglycol dimethylacrylate & EGDMA \\
\hline & Divinylbenzene & DVB \\
\hline
\end{tabular}




\section{Appendix B}

\section{Design of Flow Through Reactor}

The design of the flow through reactor is described below:

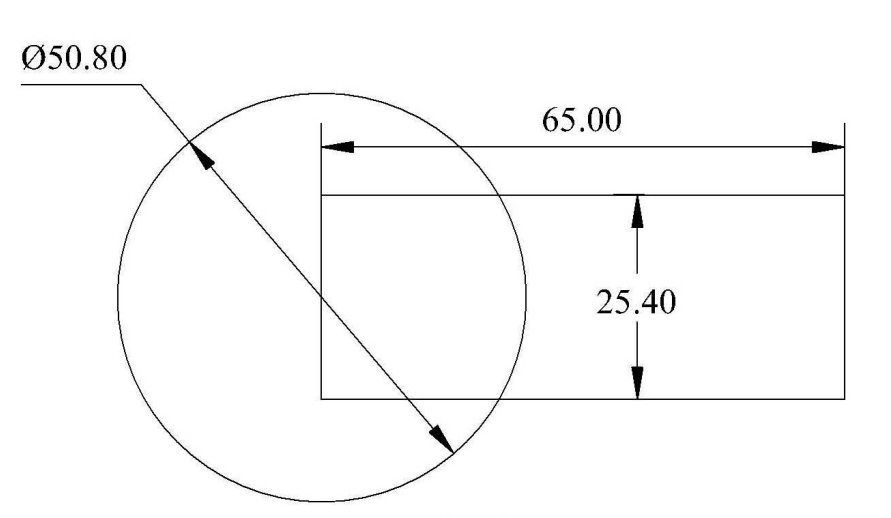

Top View

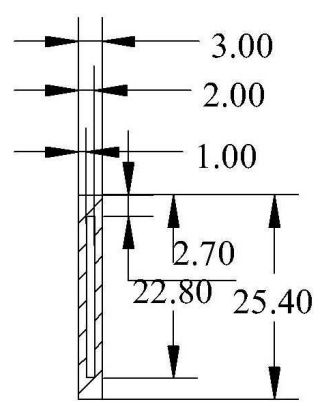

Side View 1

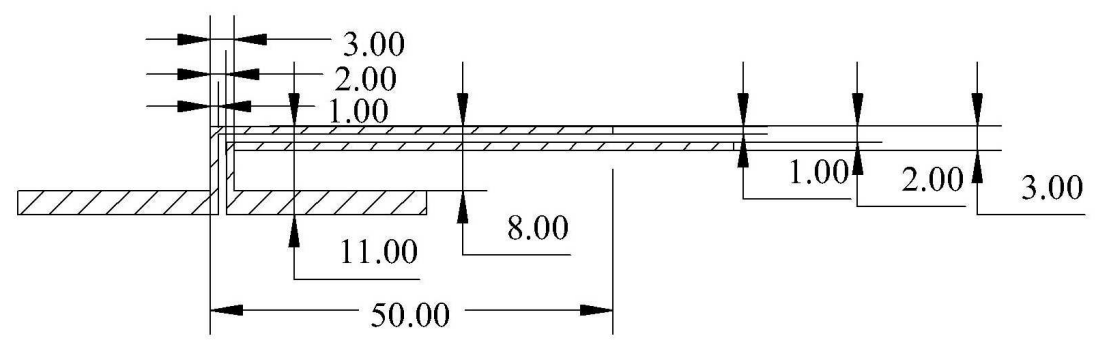

Name: Secondary chamber of the flow through reactor Material: Plexiglass for circular piece, glass for the rest

Side View 2

All units for dimensions are in millimeter

Figure B.1. Dimensions for the secondary chamber of the flow through reactor. All dimensions are in millimeters. 


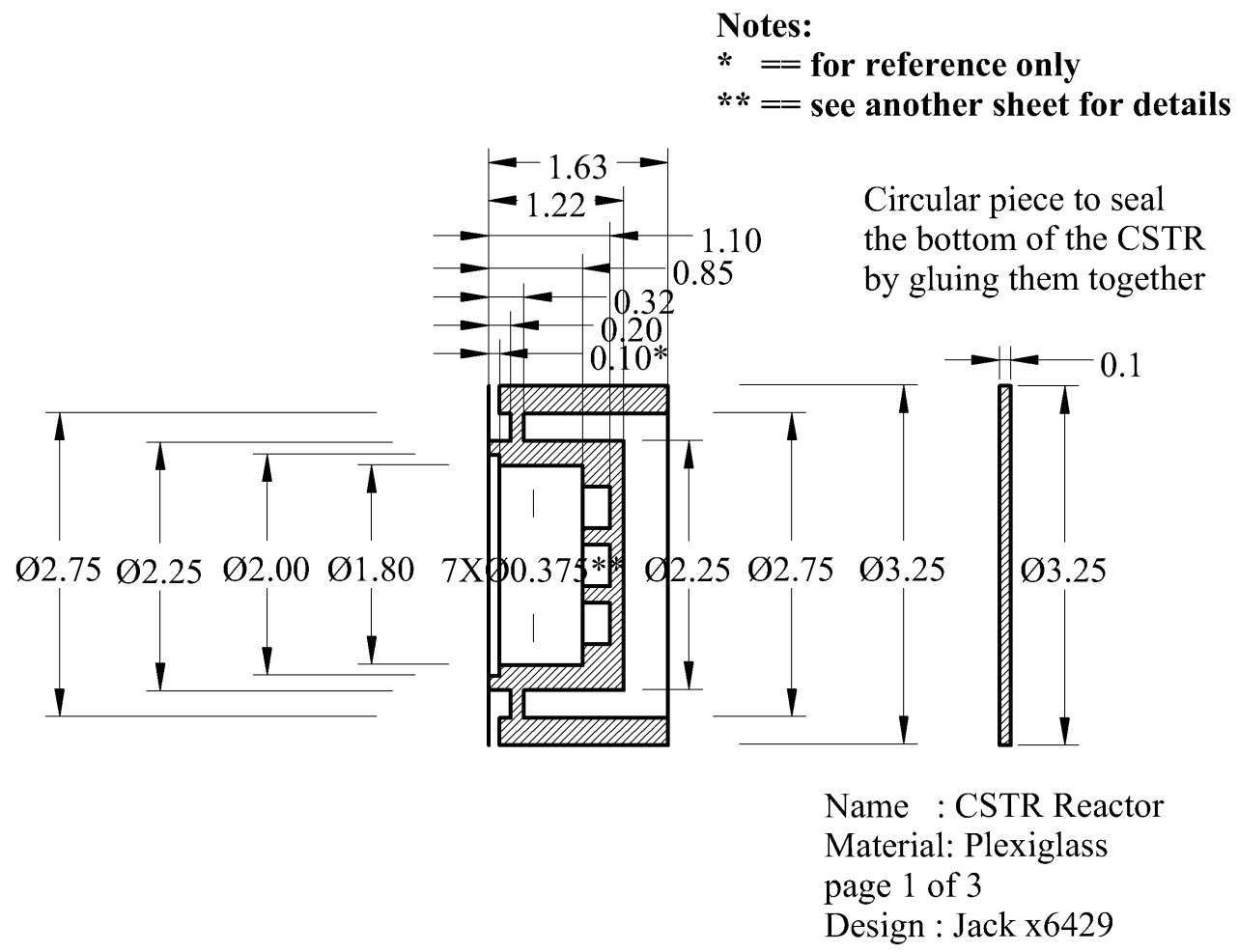

Figure B.2. Main dimensions for the primary chamber of the flow through reactor. All dimensions are in inches.

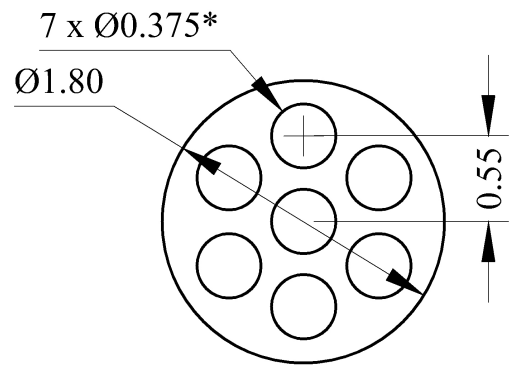

Boring pattern for the stirrer wells

* May adjust to the larger available tool size

(page 2 of 3 )

Figure B.3. Stirring wells for the primary chamber of the flow-through reactor. All dimensions are in inches. 


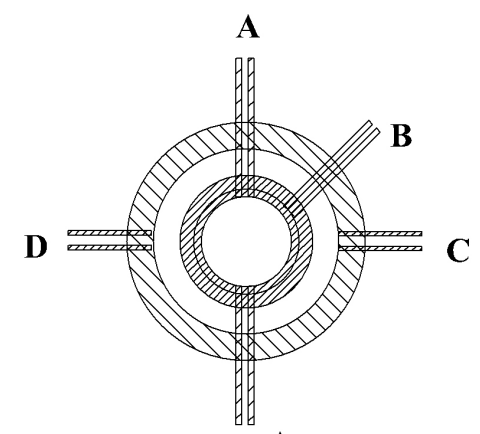

A

Special Notes:

A,B,C,E pass through both outer and inner walls

$\mathrm{D}$ and $\mathrm{F}$ pass through outer wall only to the water jacket

1. $\mathrm{A}==$ Inlets(x2), size see the tubing provided, $\sim 0.25$ "OD $\sim 0.10$ "ID

2. $\mathrm{B}==$ Electrode at the bottom, tight fit to the electrode provided, $\sim 0.067 \mathrm{inch}$

3. $\mathrm{C}==$ Water bath at the bottom, fit to the tubing provided, $\sim 0.375$ "OD $/ \sim 0.25$ "ID

4. $\mathrm{D}==$ Water bath at the top, size as $\mathrm{D}$

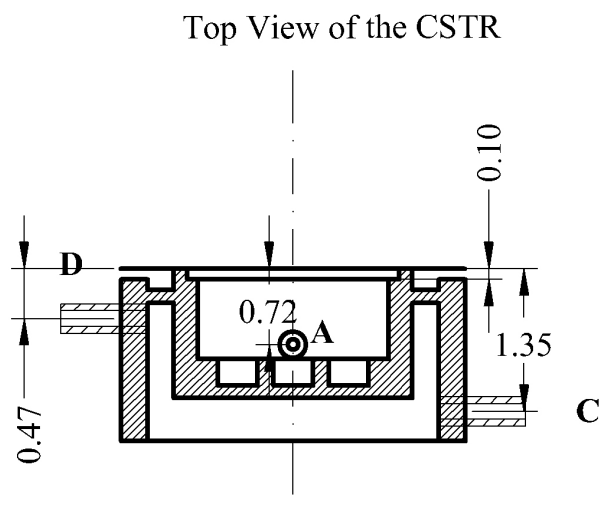

View from the A Side

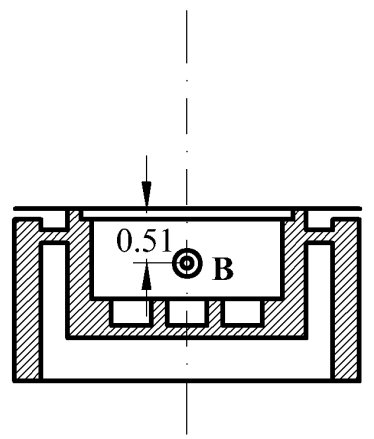

View from the B side

Name : CSTR Reactor, Inlet placement

Material: Plexiglass

Page 3 of 3

Design : Jack x6429

Figure B.4. Inlet positions for the primary chamber of the flow through reactor. All dimensions are in inches. 